\title{
5 Kommunikation in der Ausstellung: Analyse
}

Im folgenden Kapitel soll die Interaktion von Besuchern bei ihrem Gang durch das Museum analysiert und so die soziale Praxis des gemeinsamen Museumsbesuchs rekonstruiert werden.

Die Funktion dieses zweiten Analyseteils ist es nicht, den Beleg dafür zu liefern, dass die Bedeutungspotenziale im Ausstellungsraum in Kapitel 3 korrekt ermittelt worden sind. Die Frage, wie sich die Untersuchung der im Raum angelegten Bedeutungspotenziale einerseits und die Untersuchung empirischer Nutzungs- und Interpretationsvorgänge dieses Raums andererseits miteinander verbinden lassen, wird erst in Kapitel 6 beantwortet. In diesem Kapitel werde ich dagegen den im Gegenstand angelegten Relevanzen folgen und die Interaktion im Ausstellungsraum ganz eigenständig untersuchen.

Deshalb sind die Analysen im Folgenden auch nicht nach den kommunikativen Aufgaben gegliedert, die ich im vorigen Kapitel herausgearbeitet und im Detail beschrieben habe. Sie folgen vielmehr der Frage, wie die Besucher in ihrer Interaktion mit dem Raum umgehen (mehr dazu gleich). Damit sind zwei eng aufeinander bezogene Aspekte gemeint. Zum einen: wie die Besucher mit Sprache und einer Vielzahl weiterer leibgebundener Ressourcen den für die Durchführung des gemeinsamen Museumsbesuchs erforderlichen und angemessenen Interaktionsraum herstellen. Aber auch: wie die Besucher ihre räumliche Umwelt und die in ihr arrangierten Objekte auf ganz spezifische Art und Weise für ihre Interaktion im Ausstellungsraum aktivieren und wie sie dem Ausstellungsraum und seinen Objekten dabei ganz bestimmte Bedeutungen zuschreiben.

\subsection{Methode und Korpus}

Wie schon in Kapitel 1 gesagt, ist die Untersuchung der Interaktion im Ausstellungsraum in der Konversationsanalyse und ihren theoretischen Grundannahmen verankert. Diese Verankerung hat Konsequenzen für meine Fragestellung, das Verständnis meiner Daten sowie für das konkrete Vorgehen bei der Erhebung und Analyse.

Im Mittelpunkt meines Interesses steht die Frage nach den Praktiken, mit denen die Interaktionsbeteiligten ihre alltäglichen Handlungen organisieren und wie sie sie in den sich entwickelnden Interaktionskontext einbetten. Diese Praktiken gilt es durch die detaillierte Analyse der Interaktion zu rekonstruieren. Dabei gilt es, die „Teilnehmerperspektive“ einzunehmen, also das Interaktionsgeschehen in seiner Sinnhaftigkeit für die Interaktionsbeteiligten zu beschreiben, und nicht ein Modell des Interaktionsverhaltens zu konstruieren, das das Geschehen 
mit Hilfe von ,Faktoren` zu erklären versucht, die aus einer Theorie abgeleitet werden.

Grundlegend für die Rekonstruktion der Teilnehmerperspektive ist das Prinzip des „display“: Da sich die Interaktionsbeteiligten nicht ,in die Köpfe schauen können‘, müssen sie sich jederzeit gegenseitig die Bedeutung ihrer Aktivitäten und ihr Verständnis der Aktivitäten ihres Gegenübers anzeigen. Deppermann (2008: 50) beschreibt dieses Prinzip so:

Die Konversationsanalyse geht von der zentralen methodologischen Prämisse aus, daß Gesprächsteilnehmer einander aufzeigen (,display‘, z.B. Schegloff 1997), welchen Sinn und welche Bedeutsamkeit sie ihren Äußerungen [und allgemein: ihren Handlungen, W.K.] wechselseitig zuschreiben. Wäre dies nicht wenigstens teilweise und immer wieder so, wären Handlungskoordination und Verständigung unmöglich.

Die Konversationsanalyse nutzt dieses grundlegende Funktionsprinzip nun, um auf die Interpretationen der Teilnehmer zugreifen zu können:

Wenn Gespräche sorgfältig protokolliert wurden, stehen diese Aufzeigeleistungen den Gesprächsanalytikern in der gleichen Weise wie den Gesprächsteilnehmern zur Verfügung, um Gesprächsereignisse zu interpretieren. (2008: 50)

Um das display der Museumsbesucher ausnutzen zu können, um Zugang zu ihrem Verständnis und ihrer Nutzung des Raums und der Ausstellung zu erhalten, nimmt meine Untersuchung der Kommunikation in der Ausstellung ausschließlich gemeinsame Museumsbesuche in den Blick. Der Vorteil dieser Konzentration auf gemeinsame Besuche ist, dass es keiner Fragebögen oder Interviews bedarf, um das Raumverständnis der Besucher zu rekonstruieren und der Art und Weise auf die Spur zu kommen, wie sie ihren Interaktionsraum für den Museumsbesuch konstruieren. Die Daten, die zu dieser Rekonstruktion nötig sind, sind gleichsam natürlicher Bestandteil der Situation, die ich untersuchen möchte. Indem ich mit Aufnahmen von Museumsbesuchen arbeite, die auch ohne meine Anwesenheit stattgefunden hätten, entspricht meine Untersuchung der grundlegenden Forderung der Konversationsanalyse, mit natürlichen Daten zu arbeiten: Daten, die im Idealfall ohne Einwirken des Forschers entstanden sind - wir werden weiter unten sehen, wie ich diese Forderung bei der Erhebung der Videoaufnahmen berücksichtigt habe - und die in authentischen Interaktionssituationen entstanden sind, also nicht in Experimentalsituationen, deren Komplexität auf die Beantwortung einer punktuellen Fragestellung hin reduziert worden ist.

Diese Besuche habe ich auf Video aufgenommen (zum genauen Vorgehen s.u.), um möglichst viel von dem mich interessierenden Interaktionsgeschehen dokumentieren zu können. Videoaufnahmen ermöglichen es nämlich, das Zusammen- 
spiel von Sprache, anderen körpergebundenen Kommunikationsressourcen und von Elementen der räumlichen Umwelt im Entstehungszusammenhang der Interaktion untersuchen zu können. Die Dokumentation des Interaktionsgeschehens per Video ermöglicht nicht nur ein beliebig häufiges Abspielen des Videos, es ermöglicht zusätzlich eine Verlangsamung der Wiedergabe, die es erlaubt, Details der Interaktion systematisch in den Blick zu nehmen, die bei Originalgeschwindigkeit schnell von anderen Phänomenen überdeckt würden. So erlauben Videoaufnahmen eine hochdetaillierte Analyse der untersuchten Interaktionen. Das Arbeiten mit Videoaufnahmen hat daher in der letzten Zeit in den Sozial- und Geisteswissenschaften massive Verbreitung gefunden (s. etwa Mondada 2013, Heath/Hindmarsh/ Luff 2010, Bohnsack 2009, Kissmann 2009 oder Goldman et al. 2007 für Überblicke mit je unterschiedlicher Schwerpunktsetzung; zu Vorteilen des Arbeitens mit Video im Museumszusammenhang s. vom Lehn/Heath/Hindmarsh 2001: 194-196).

Wie kann nun untersucht werden, wie die Besucher den Ausstellungsraum und seine „socially sedimented structure“ (Goodwin 2000: 1490) für ihre soziale Praxis des gemeinsamen Museumsbesuchs nutzen und wie sie dabei die Vielzahl der im Raum präsenten dauerkommunikativen semiotischen Ressourcen (die in Kapitel 3 untersucht worden sind) in ihre anwesenheitsbasierte Interaktion integrieren? Wie im vorigen Kapitel dargestellt, gehe ich davon aus, dass die räumliche Umwelt nicht die Interaktion determiniert, die in ihr stattfindet, sondern dass Raum in der Interaktion hergestellt und mit Bedeutung versehen wird. Das gilt auch für den Ausstellungsraum.

Wie aber kann man beobachten, wie die Teilnehmer ihre räumliche Umgebung als Ausstellungsraum hervorbringen und wie sie den Raum und die in ihm präsentierten Objekte für ihre Interaktion mit Bedeutung versehen? Die Art und Weise, wie die Interaktionsbeteiligten ihre räumliche Umwelt für ihren gemeinsamen Museumsbesuch konstruieren, wird beobachtbar, weil die Besucher die Museumsausstellung nicht alleine durchschreiten, sondern als Paar oder Gruppe. Damit stellt sich ihnen die Aufgabe, für ihre Begleiter ihre Interpretation ihrer räumlichen Umwelt sicht- und hörbar werden zu lassen. Damit der Museumsbesuch als gemeinsame Aktivität gelingen kann, müssen die Besucher sich mit ihren Begleitern koordinieren (die „interpersonelle Koordination“ sensu Deppermann/ Schmitt 2007). Es reicht nicht aus, dass ein Besucher stehen bleibt, um sich die Exponate in einer Vitrine anzuschauen. Er muss sein Stehenbleiben so gestalten, das es von seinem Begleiter als Aktivität im Rahmen des gemeinsamen Museumsbesuchs erkannt werden kann - also als Stehenbleiben, um sich ein Exponat näher anzuschauen (und nicht etwa als Stehenbleiben, um sich auszuruhen). Wir als Beobachter haben einen Zugang zu den Interpretationen der Akteure, weil sie sich ihre Interpretationen als Grundlage für ihre Interaktion gegenseitig aufzeigen (müssen). Anders als beim stillen Lesen eines Buchs, werden die Raum- 
Interpretationen unmittelbar sichtbar, und zwar in der Art und Weise selbst, wie die Besucher den Raum nutzen. In aller Regel vollzieht sich das nicht in speziellen Handlungen, die den Interaktionspartner über die Raum-Interpretation des Handelnden informieren. Vielmehr wird die Raum-Interpretation im Vollzug des gemeinsamen Ausstellungsbesuchs sichtbar, als untrennbarer Bestandteil dieser Handlungen (das ist ihr dokumentarischer Charakter).

Die Analyse der Raumherstellung durch die Museumsbesucher erfolgt in drei Schritten, die sich an den von Heiko Hausendorf (2010) rekonstruierten Teilaufgaben der „Situierung“ orientieren, mit denen die Interaktionspartner die räumlichen Voraussetzungen für ihre Interaktion hervorbringen und ihre Interaktion in der räumlichen Umwelt verankern: nämlich der Herstellung des „Bewegungs-“, des „Wahrnehmungs-“ sowie des „Handlungsraums“ (s. im Detail 4.2.2). Meine Untersuchung der Raumkonstitution in der Kommunikation in der Ausstellung geht dabei immer zweischrittig vor. Im ersten Schritt untersuche ich, wie die Besucher einen gemeinsamen Bewegungs-, Wahrnehmungs- und Handlungsraum herstellen, indem sie ihre Bewegungen aufeinander ausrichten, ihre visuelle Aufmerksamkeit aufeinander abstimmen und ihre Handlungen so ausführen, dass diese als gemeinsame Handlungen erkennbar sind und die Interaktionsteilnehmer räumlichen Zugang $\mathrm{zu}$ diesen Handlungen haben. Im zweiten Schritt untersuche ich die Musterhaftigkeit dieser Teil-Räume: Was ist das Museumsspezifische ihrer Herstellung? Was macht einen gemeinsamen Bewegungsraum wiedererkennbar zu einem musealen Bewegungsraum? Was ist das Charakteristische des von den Besuchern interaktiv hergestellten musealen Wahrnehmungsraums? Und wie gestalten die Besucher musterhaft ihren gemeinsamen Handlungsraum, so dass dieser als musealer Handlungsraum zu erkennen ist? Hierbei geraten Fragen in den Blick wie die folgenden:

- Wie genau konstruieren die Besucher den Ausstellungsraum im Hinblick auf die Bewegungen, die dieser erlaubt oder erforderlich macht? Wo(hin) gehen sie durch den Raum? Wie gestalten sie ihre Bewegungen durch den Raum und welchen museumsspezifischen Sinn weisen sie diesen Bewegungen zu?

- Welche ihrer Wahrnehmungen bringen die Besucher in der Ausstellung zur Deckung und welche Elemente der räumlichen Umwelt befinden sich im Zentrum ihrer visuellen Aufmerksamkeit? Wie gestalten sie ihre Wahrnehmungen auf museumsspezifische Weise und welche Bedeutung haben diese Wahrnehmungen für sie?

- Wie nutzen die Besucher den Ausstellungsraum für ihre Handlungen und was sind die museumsspezifischen Handlungen, für die sie den gemeinsamen Handlungsraum konstruieren? 
Die diesem zweiten Analyseschritt zugrundeliegende Idee ist, dass es die musterhafte Hervorbringung dieser drei Teil-Räume des Interaktionsraums ist, die zusammen für die Erkennbarkeit des gemeinsamen Museumsbesuchs verantwortlich ist.

Dass es möglich ist, über die Beschreibung der Musterhaftigkeit der interaktiven Raumkonstruktion etwas Substanzielles über die soziale Praxis des gemeinsamen Museumsbesuchs zu erfahren, hat zwei Gründe. Zum einen die prinzipielle Reflexivität der Interaktion, wie sie von Ethnomethodologie und Konversationsanalyse herausgearbeitet worden ist (s. Auer 1999): Die Interaktion ist ,kontextsensitiv“ und ,Kontext generierend', das heißt, dass die Interaktionsbeiträge auf den jeweiligen (emergierenden) Kontext zugeschnitten sind und ihren Sinn aus ihrer Passung mit diesem Kontext beziehen, dass dieser Kontext aber gleichzeitig durch diese Interaktionsbeiträge hervorgebracht wird. Indem sie also ihre gemeinsamen Bewegungs-, Wahrnehmungs- und Handlungsräume auf den Ausstellungsraum und seine Nutzung im Rahmen der Praxis des gemeinsamen Museumsbesuchs zuschneiden, bringen die Interaktionsbeteiligten ihre räumliche Umwelt als Ausstellungsraum hervor. Der zweite Grund liegt in der Tatsache, dass der hier untersuchte Typ von Interaktion raumgebunden ist. Mit anderen Worten: Die spezifische Nutzung der räumlichen Umwelt - konkret die Nutzung des Ausstellungsraums und der in ihm präsentierten Exponate als dauerkommunikatives Kommunikationsangebot - spielt für die Praxis des gemeinsamen Museumsbesuchs eine so zentrale Rolle, dass man mit der Beschreibung der musterhaften Konstruktion des Bewegungs-, Wahrnehmungs- und Handlungsraums für den gemeinsamen Museumsbesuch die konstitutiven Besonderheiten dieses Typs von Interaktion im Kern erfasst. Das heißt nun aber keineswegs, dass Sprache für die Rekonstruktion dieser sozialen Praxis eine untergeordnete (oder möglichweise gar keine) Rolle spielte. Zwar gibt es durchaus längere Phasen, in denen sich die Besucher in einem „open state of talk“ (Goffman 1981: 134) befinden, aber eben nicht sprechen. Und doch werden wir sehen, dass nicht nur bei der Herstellung des musealen Handlungsraums (mit seinem Fokus auf der interaktiven Erarbeitung von geteiltem Wissen), sondern auch schon bei der Herstellung des musealen Bewegungsraums Sprache immer mitberücksichtigt werden muss - etwa wenn man fragt, wie die Besucher aushandeln, was ein legitimes Bewegungsziel im Rahmen ihres Museumsrundgangs ist. Weil Sprache und andere Ausdruckformen in der hier untersuchten Praxis aufs Engste miteinander verwoben sind, werde ich auch nicht erst das visuell Wahrnehmbare untersuchen, um dann Sprache ,ergänzend‘ dazuzunehmen (vgl. die „mikrophänomenologische Perspektive“ in Deppermann/Schmitt 2007: 38), sondern von Vornherein alle den Teilnehmern zugänglichen Sinneseindrücke - sofern sie über die Videoaufnahme zugänglich sind - zugleich analysieren. 
Die Untersuchung der Kommunikation in der Ausstellung in Kapitel 5 basiert auf einem Videokorpus, das ich im Zoologischen und Paläontologischen Museum der Universität Zürich aufgenommen habe. ${ }^{1}$ Das Korpus besteht aus ca. 80 Aufnahmen von einer Gesamtlänge von über 10 Stunden. Der Großteil der Aufnahmen dokumentiert ungesteuerte Museumsbesuche von Zweiergruppen, aber auch von Gruppen bis zu ca. fünf Personen. Unter diesen Gruppierungen gibt es Mütter, Väter oder andere Bezugspersonen mit Kindern, Großmütter oder -väter mit Enkel oder Enkeln, Ehepartner, jugendliche oder erwachsene Freundesgruppen, Gruppen aus Kinderkrippen oder Hortgruppen mit ihren jeweiligen Betreuungspersonen. Damit decken die Aufnahmen meines Korpus die Vielfalt der Besucher und Besuchergruppen ab, die in den von mir ausgewählten Museen zu beobachten ist. Der Umfang des Korpus und die Variationsbreite der dokumentierten Besuchsvorgänge garantieren, dass das interessierende Phänomen der Kommunikation in der Ausstellung in allen relevanten Facetten abgebildet ist. Das gilt allerdings nur für den von mir untersuchten Typ von Museum - das traditionelle naturkundliche oder naturwissenschaftliche Museum - „traditionell“ als Gegenbegriff zu „interaktiven“ Museen wie den Science Centers (die Gegenstand von Kesselheim/Brandenberger/Hottiger 2021 oder Kesselheim/Brandenberger 2021 sind). Eine weitere Einschränkung bezieht sich auf die Nutzung multimedialer Angebote im Ausstellungsraum (PC-Informationsstationen, PC-Ratespiele usw.). Zwar habe ich einige Fälle der Nutzung dieser Elemente dokumentiert, aber eine Analyse, die sich gezielt mit der Rolle multimedialer Elemente im Ausstellungsraum beschäftigt, bräuchte auf diesen spezifischen Fall angepasste Dokumentationsmethoden. Wollte man aber die Benutzereingaben in Form eines Screencasts dokumentieren, müsste man entweder für jede PC-Station ein eigenes Screencastprogramm einrichten (und die Daten dann mit den Videodaten synchronisieren) oder man müsste die Auswahl- und Bewegungsfreiheit der Besucher beschränken, was gegen die Idee spricht, die soziale Praxis des Museumsbesuchs möglichst unbeeinflusst zu dokumentieren (Fu et al. 2019, Allen/Peterman 2019: 19). Wichtiger als diese technischen Probleme aber scheint mir zu sein, dass sich die Nutzung multimedialer Angebote im Ausstellungsraum massiv auf die räumlichen Konfigurationen der Museumsbesucher auswirkt (einen Eindruck aus der Perspektive der Besucherforschung gibt Drotner et al. 2019). Es bietet sich also an - gerade auch vor dem Hintergrund der intensiven Diskussion um die Digita-

1 Ich danke Marianne Haffner, der Leiterin des Zoologischen Museums, und Heinz Furrer, dem Leiter des Paläontologischen Museums, für die Erlaubnis, in ihren Räumen filmen zu können. Den Mitarbeiterinnen des Museums danke ich für die freundliche Aufnahme und engagierte Unterstützung bei meiner Erhebung. 
lisierung im Museum - die Untersuchung dieses Aspekts in eine eigene Untersuchung auszulagern.

Zur Datenerhebung. Um möglichst die gesamte Breite des Museumspublikums zu erfassen, wurden Gruppen von unterschiedlicher Größe (Paare, Familien, Freundesgruppen, Horte usw.), unterschiedlicher Zusammensetzung (Erwachsene, Erwachsene mit jüngeren oder älteren Kindern, ältere Kinder) in möglichst allen Bereichen des Museums gefilmt, wobei die Aufnahmen über die Woche und über unterschiedliche Tageszeiten gestreut wurden. Die Aufnahmen geben also einen guten Querschnitt über die in den Museen präsenten Besuchergruppen wieder. Trotz alledem: Eine Repräsentativität der aufgenommenen Besucher nach externen sozialen Faktoren wie Geschlecht, nationale oder regionale Herkunft, Bildungsgrad, Vertrautheit mit den ausgestellten Disziplinen usw. wurde nicht angestrebt. Dies ergibt sich aus der konversationsanalytischen Überzeugung, dass nur solche (und genau diejenigen) sozialen Kategorien in der Analyse zu berücksichtigen sind, deren Relevanz sich die Teilnehmer in den Daten anzeigen. Ob also ein Besucher das erste Mal mit Fossilien konfrontiert ist oder ob er auf dem Gebiet Experte ist, zeigt sich in den Daten, wenn es für die Teilnehmer selbst relevant ist. Das Gleiche gilt für Herkunftskategorien: Ob es sich bei den Besuchern um Einheimische handelt, um Touristen oder Zugezogene, hat nur dann Relevanz, wenn diese sozialen Kategorien in den Daten sichtbar oder hörbar eine Rolle spielen. Deshalb habe ich keine demographischen Daten zu den von mir Gefilmten erhoben. ${ }^{2}$

Trotz dieser Bemühungen gibt es dennoch es einen klaren bias im Hinblick auf die Häufigkeit, mit der Kinder an den dokumentierten Interaktionen beteiligt sind. Dies hängt damit zusammen, dass Kinder (bis hin zum präverbalen Alter) im Zoologischen und Paläontologischen Museum der Universität Zürich eine bedeutende Besuchergruppe sind. Doch liegt in der großen Zahl von Aufnahmen mit Kindern nicht nur die Gefahr einer Verzerrung, sondern auch eine Chance für die Analyse. Da Kinder häufig noch nicht vollständig in den Umgang mit Ausstellungen im Museum einsozialisiert sind, treten viele der Regeln, die sonst stillschweigend vorausgesetzt werden, in der Interaktion mit Kindern häufig zutage, bis dahin, dass sie zum Thema ausführlicher Erklärungen werden. Die Momente, in denen Kindern der angemessene Umgang mit der Ausstellung nahegebracht wird oder in denen ihr unangemessenes Verhalten korrigiert wird, können für die Analyse wertvolle Einstiegspunkte darstellen, von denen aus sich Merkmale

2 Überdies bin ich hier an Generalisierungen vom Typ „Besucher mit Hochschulabschluss stellen mehr Fragen als Besucher aus bildungsfernen Schichten“ nicht interessiert. Für die Beantwortung dieser Fragen wären demographische Daten natürlich unerlässlich. 
erschließen lassen, die für die Praxis des gemeinsamen Museumsbesuchs allgemein gelten, die aber durch die langjährige Routine im Umgang mit der Museumsausstellung als kommunikativem Angebot im Normalfall an der kommunikativen Oberfläche nicht oder nur schwer nachweisbar sind.

Bei den Aufnahmen meines Korpus handelt es sich um zehn- bis fünfzehnminütige Ausschnitte aus ungesteuerten Museumsbesuchen. Ich habe mich Besuchern, die schon längere Zeit die Ausstellung durchlaufen haben, als Sprachwissenschaftler vorgestellt, der daran interessiert sei, wie ein „ganz normaler Museumsbesuch“ funktioniere, und sie gefragt, ob ich sie „zehn Minuten mit meiner Kamera bei ihrem Besuch begleiten“ dürfe. Der Hinweis darauf, dass mit der Teilnahme an meiner Untersuchung keine spezielle Aufgabe, keine Beschränkung der Bewegungsfreiheit und weder ein anschließender Test noch eine Befragung verbunden sein würden, dürfte dazu beigetragen haben, dass die überwiegende Zahl der Angefragten einer Aufnahme zugestimmt haben. ${ }^{3}$

Das Aufnehmen der Kommunikation in der Ausstellung ist technisch anspruchsvoll:

The environment of the museum floor presents a set of challenges beyond those inherent in most laboratory or school settings. First, the acoustics of the public spaces are very poor, and there is a huge amount of ambient noise. Major sources of sound include background hubbub from distant visitors, screams from excited children, conversations by nearby visitors (easy to mistake for members of the study group), and the myriad of sounds that interactive exhibits make when in use, such as bangs, clings, music, ratcheting, splashes, music, and prerecorded speech. [...] Second, visitors move around a great deal, and the groups they arrive in keep changing and reforming on short time-scales. (Allen 2002: 264f.)

Deshalb schließt die Autorin:

We considered videotaping to get the most precise information about visitors' movements, but eventually dismissed this as unfeasible. (Allen 2002: 266)

3 Die Einverständniserklärung habe ich den Teilnehmenden jeweils am Ende einer Aufnahme ausgeteilt. Für die restlichen Besucher war während der gesamten Erhebung am Informationstresen neben dem Museumseingang ein Schild angebracht, das auf die Aufnahmen hinwies (vgl. Gutwill 2002). Auf den Schildern wurde den Besuchern die Möglichkeit eingeräumt, jederzeit einer Aufnahme zu widersprechen oder die Löschung ihrer Daten zu verlangen. Wie im Fall der Studien von vom Lehn, Heath und Kollegen erkundigten sich einzelne Besucher nach den Zielen meiner Untersuchung, aber kein Besucher widersprach der Aufnahme (vgl. z.B. vom Lehn/ Heath/Hindmarsh 2001: 194-196). 
Mit dem heutigen Stand der Aufnahmetechnik sind Videoaufnahmen in Ausstellungen zwar weiterhin anspruchsvoll, aber - wie die wachsende Zahl von Videostudien belegt - nicht mehr unmöglich.

Bei der Erhebung der Daten habe ich versucht, die Natürlichkeit der Situation möglichst wenig zu beeinträchtigen. Deshalb habe ich mit einer kleinen Handkamera gefilmt, bei starkem Publikumsverkehr und daher großem Umgebungsschall unter Zuhilfenahme eines aufgesteckten Richtmikrofons. Bei der Bewegung der gefilmten Gruppen durch das Museum habe ich jeweils soviel Abstand eingehalten, dass die Gruppe als Ganzes zu sehen war, aber die Tonqualität befriedigend blieb. In den Daten findet man punktuelle Hinweise darauf, dass die Kamera und der Kameramann für die Gefilmten für einen Moment bedeutsam werden: ein Blick in die Kamera, gerade bei Kindern (oder seine auffällige Vermeidung bei Erwachsenen), ein Öffnen der räumlichen Formation, um ein ,Hineinfilmen` zu erlauben, ein kurzer ,Schlenker‘ auf dem Weg zum nächsten Exponat, mit dem man die Kollision mit dem Filmenden vermeidet, ein Absenken der Sprechlautstärke, mit dem man ihn aus dem Kreis der Zuhörer ausschließt usw. ${ }^{4}$ In aller Regel nimmt die Zahl dieser Indizien mit fortschreitender Aufnahmedauer schnell ab. Der Museumsbesuch wird anscheinend von vielen als Interaktion im öffentlichen Raum aufgefasst, die einen geringen Grad an Privatheit aufweist. Ein Beleg dafür scheint mir auch die oben erwähnte unkomplizierte Bereitschaft zu sein, sich beim Museumsbesuch filmen zu lassen.

Insgesamt bin ich daher überzeugt, mit meinem Erhebungsdesign eine gute Balance zwischen den Polen maximale Qualität der Daten und maximale Natürlichkeit der Situation gefunden zu haben. Trotz alledem gilt es, bei der Arbeit mit meinem Videokorpus im Blick zu behalten, dass die Videodaten nicht mit dem Interaktionsgeschehen gleichgesetzt werden dürfen, das sie dokumentieren. So schreibt Erickson (2007: 153):

[V]ideotapes are better regarded as sources for data than as data in themselves. [...] From such records, data can be defined, analytically. But it seems to me that it is naive realism to think of them as data themselves.

Im Prozess des Filmens kommt es zu einigen folgenreichen Veränderungen: Das Filmen erfolgt von einem Standpunkt aus, der sich nicht aus der Einnahme einer bestimmten Rolle in der Interaktionssituation ergibt (also: Mitglied der gleichen Besuchergruppe, ,fremder‘ Besucher, Museumsmitarbeiter usw.), sondern auf-

4 Zur Rolle der Kamera bei gesprächsanalytischen Untersuchungen im öffentichen Raum: Frers (2009). 
grund von technischen Beschränkungen (Brennweite, Mikrofonempfindlichkeit), die Aufnahmen reduzieren die ursprünglichen Wahrnehmungen auf den visuellen Kanal und erfolgen in 2-D. Der Bildrahmen ist enger als das menschliche Gesichtsfeld, und er suggeriert, dass alles, was in dem Rahmen zu sehen ist, in irgendeiner Form zusammengehört, ohne dass es in der Realität etwas Analoges gäbe. Die Analyse muss ihren Umgang mit den Daten also unbedingt kritisch reflektieren.

Abgesehen von diesen Unterschieden, die durch den Einsatz eines Aufnahme- und Speichermediums bedingt sind, darf eine prinzipielle Tatsache nicht vergessen gehen: nämlich dass die Aufnahme kein mechanisches Registrieren der sozialen Realität ist. Was auf den Bildern zu sehen ist, spiegelt die ,Live'-Interpretation des interaktiven Geschehens durch den Filmenden wieder, der bestrebt ist, das auf dem Videobild sichtbar werden zu lassen, was er als relevanten Teil der ablaufenden Interaktion versteht. So kann man immer wieder in den Daten sehen, wie sich die Kamera den Gefilmten nähert, wenn diese vor einer Vitrine zum Stillstand kommen - offenbar als Reaktion auf die Vermutung, dass sich eine stationäre Phase des Museumsbesuchs anschließen wird - oder wie sich die Kamera entfernt, wenn der Kameramann augenscheinlich zum Schluss gekommen ist, dass die stationäre Phase sich auflöst. Trotz all dieser Einschränkungen scheinen mir die Videoaufnahmen ein reiches und präzises Bild des interaktiven Geschehens zu bieten, das über das mit anderen Methoden wie Fragebögen oder Interviews Erhebbare (vgl. 4.1) weit hinausgeht, solange in der Untersuchung die Entstehung der Daten im Prozess der Analyse reflektiert wird (s. Kesselheim 2016 zu unterschiedlichen Auffassungen zum Status der Daten in der videobasierten Raumanalyse).

Wie können die Videodaten nun im Rahmen dieses gedruckten Texts präsentiert werden? Anders als im Fall der Fotografien ist hierzu ein weiterer Bearbeitungsschritt erforderlich: das Erstellen einer Transkription. ${ }^{5}$ Der Transkription hilft dabei, die präzise sequenzielle Organisation der interessierenden - prinzipiell flüchtigen - Handlungen besser beobachten zu können.

The transcription provides an important vehicle for becoming familiar with the complexities of a particular fragment and for beginning to explicate the relations between actions and activities. It also provides an important resource for documenting observations and recalling insights and analytic observations. (Vom Lehn/Heath 2007b: 292)

5 Beim Erstellen und Korrigieren der Transkripte haben mich zu unterschiedlichen Zeitpunkten meiner Erhebung unterstützt (in alphabetischer Reihenfolge): Daphni Antoniou, Tobias Funk, Sabina Gröner, Evelyn Kähler, Hiloko Kato, Nicolas Wiedmer und Barbara Zeugin. 
Obwohl ich für meine Untersuchung gleich nach der Erhebung von allen Aufnahmen Rohtranskripte erstellt habe, wurden viele Analysen direkt im Videoschnittprogramm vorgenommen: durch Markieren, Ausschneiden oder Untertiteln relevanter Ausschnitte. Die Transkripte, die sich in dieser Arbeit befinden, entstanden erst in späteren Phasen der Analyse, auf dem Weg zur zunehmenden Verdichtung und Versprachlichung der Analysebeobachtungen. Sie sind deshalb erkennbar darauf zugeschnitten, die analytischen Aussagen nachvollziehbar zu machen.

Um eine möglichst einfache Lesbarkeit über disziplinäre Grenzen hinweg zu ermöglichen, folge ich nicht der in der multimodalen Gesprächsanalyse gängigen Konvention von Mondada (2016). Stattdessen wird das körperlich-räumliche Geschehen durch Standbilder zugänglich gemacht, deren Position im Interaktionsablauf über das Zeichen „\#“ und eine Identifikationsnummer erschlossen werden kann. ${ }^{6}$ Bei der Wiedergabe des verbalen Anteils der Interaktion orientiere ich mich an der im deutschsprachigen Raum etablierten Konvention „GAT 2“ (Selting et al. 2009). Die folgende Liste gibt die wichtigsten Transkriptionskonventionen wieder (vgl. Selting et al. 2009: 391f.):

\section{Sequenzielle Struktur / Verlaufsstruktur}

\begin{tabular}{lll}
\hline[ & Überlappungen und Simultansprechen \\
{[} & ] & \\
$=$ & $\begin{array}{l}\text { schneller, unmittelbarer Anschluss neuer Spre- } \\
\text { cherbeiträge oder Segmente (latching) }\end{array}$ \\
\hline
\end{tabular}

\section{Pausen}

(.) $(-)(--) \quad(--)$

(2.5)
Mikropause, Pausen von ca. $0.2-0.5,0.5-0.8$, $0.8-1.0$ Sek. Dauer

Pausen über 1 Sek. Dauer

\section{Ein- und Ausatmen}

${ }^{\circ} \mathrm{h}{ }^{\circ} \mathrm{hh}{ }^{\circ} \mathrm{hhh} / \mathrm{h}^{\circ} \mathrm{hh}^{\circ} \mathrm{hhh}^{\circ}$

Ein- bzw. Ausatmen (Dauer wie bei Pausen)

6 Zum Status der Standbilder und ihrem analytischen Wert: Hausendorf (2012: 145-147), Bohnsack (2009: 151-158). 


\begin{tabular}{ll} 
Akzentuierung & \\
\hline akZENT & Fokusakzent \\
akzEnt & Nebenakzent \\
ak!ZENT! & extra starker Akzent
\end{tabular}

\section{Tonhöhenbewegung am Ende von Intonationsphrasen}

\begin{tabular}{|c|c|}
\hline$?$ & hoch steigend \\
\hline , & mittel steigend \\
\hline- & gleichbleibend \\
\hline ; & mittel fallend \\
\hline . & tief fallend \\
\hline \multicolumn{2}{|l|}{ Sonstiges } \\
\hline und_äh & Verschleifungen innerhalb von Einheiten \\
\hline äh öh äm & Verzögerungssignale, sog. „gefüllte Pausen“ \\
\hline$::::::$ & Dehnung (Dauer wie oben bei Pausen) \\
\hline ? & Abbruch durch Glottalverschluss \\
\hline ( (hustet)) & Para- und Außersprachliches \\
\hline$<<$ hustend $>>$ & Para- und Außersprachliches, sprachbegleitend \\
\hline$<<$ erstaunt $>>$ & interpretierende Kommentare mit Reichweite \\
\hline \multicolumn{2}{|l|}{ Transkriptionsprobleme, Auslassungen } \\
\hline$(\quad)$ & unverständliche Passage ohne weitere Angaben \\
\hline$(x x x),(x x x \quad x x x)$ & ein bzw. zwei unverständliche Silben \\
\hline (solche) & vermuteter Wortlaut \\
\hline (solche/welche) & mögliche Alternativen \\
\hline ((unverständlich, ca. 3 Sek)) & unverständliche Passage mit Dauer \\
\hline$((\ldots))$ & Auslassung im Transkript \\
\hline
\end{tabular}


Um eine einfache Lesbarkeit der Transkripte zu gewährleisten, werden in GAT 2 die Gesprächsbeiträge nicht phonetisch transkribiert, sondern in einer Schreibweise, die versucht, das Gesagte mit den Mitteln einer an den Höreindruck angepassten Standardorthografie wiederzugeben. Das bringt im Fall meines Korpus besondere Probleme mit sich, denn die meisten der von mir aufgenommenen Besucher sprechen einen Schweizerdeutschen Dialekt. Das Schweizerdeutsche weicht so stark vom Standarddeutschen ab, dass eine standarddeutsche Wiedergabe der schweizerdeutschen Gesprächsbeiträge - die eher einer Übersetzung gleichkäme - keinesfalls infrage kommt (wie schon der einführende Transkriptausschnitt in Kapitel 1 gezeigt haben dürfte). Zudem gibt es keine praktikable schweizerdeutsche Schriftnorm, auf die ich mich bei der Transkription stützen könnte. Meine Wiedergabe dialektaler Sprechweise versucht, gleichzeitig die Klippe einer klischierten Überbetonung des Dialekts (wie in den Dialekt-Ausgaben von Asterix) sowie die Klippe einer Einebnung der dialektalen Besonderheiten zu umgehen, und greift dazu zugegebenermaßen häufig auf ad-hoc-Lösungen zurück.

Die Analyse der Videodaten erfolgt nach den Methoden der Konversationsanalyse. Sie fusst auf feinkörnigen Analysen von Datenausschnitten, die in ihrer jeweiligen Fallspezifik ernst genommen, und nicht als bloße Belege für aus einer Theorie abgeleitete Hypothesen verstanden werden. Da die Konversationsanalyse davon ausgeht, dass die sequenzielle Struktur der Interaktion grundlegend für die Hervorbringung sozialer Ordnung ist und die Bedeutung der einzelnen Interaktionsphänomene immer nur in ihrem sequenziellen Kontext ausgeschöpft werden kann, folge ich bei den Analysen dem sich entwickelnden Interaktionsgeschehen grundsätzlich Schritt für Schritt.

Den Einstiegspunkt für die Analyse stellten einzelne Fälle dar, in denen sich die Besucher besonders intensiv mit dem Kommunikationsangebot im Ausstellungsraum auseinandersetzen, Fälle, die in irgendeiner Hinsicht als besonders ,sprechend“ oder ,typisch' erschienen, oder Fälle in denen die Regeln der Nutzung des Ausstellungsraums im Rahmen des gemeinsamen Museumsbesuchs explizit thematisiert wurden. Ausgehend von den Phänomenen, die in diesem ersten analytischen Zugriff auffällig geworden sind, wurden Kollektionen von Fällen erstellt, in denen die gleichen Phänomene - in gleicher oder ähnlicher Form - wieder auftraten, und diese Fälle ebenfalls analysiert. Gleichzeitig wurde das Teilkorpus der anfangs analysierten Fälle in mehrere Richtungen hin erweitert. Zum einen wurden die zunächst analysierten Ausschnitte durch Fälle ergänzt, in denen der Museumsbesuch gerade nicht intensiv, sondern nebenbei, etwa als Hintergrund für ein gemeinsames Gespräch vonstatten ging, sowie abweichende Fälle (,deviant cases“), in denen die Interaktionsbeteiligten das Vorliegen eines Problems manifestierten, das es für die Durchführung ihres gemeinsamen Museumsbesuchs zu bearbeiten (,reparieren`) galt. Durch die Analyse von derartigen Fällen 
treten oftmals die den ,unproblematischen` Fällen zugrundeliegenden Regelhaftigkeiten besonders deutlich hervor. Der Einbezug immer weiterer Fälle führt schließlich dazu, dass sich langsam die grundlegenden Muster herausschälen, die für die soziale Praxis des gemeinsamen Museumsbesuchs konstitutiv sind. ${ }^{7}$

Aus Platzgründen ist es in der Präsentation meiner Analysen im folgenden Abschnitt nicht möglich, ein Phänomen durch eine gesamte Kollektion hindurch zu verfolgen und so seine allgemeine Verbreitung im Korpus zu belegen. Die Phänomene, die ich anhand der einzelnen, exemplarischen Fälle beschreibe, die in das folgende Kapitel aufgenommen worden sind, sind jedoch mit Hilfe des hier beschriebenen Verfahrens rekonstruiert worden und können insofern für die von mir erhobenen Daten als repräsentativ betrachtet werden.

\subsection{Die Herstellung des musealen Bewegungsraums}

\subsubsection{Ausgangspunkt}

Die kommunikative Nutzung des Ausstellungsraums ist auf die Bewegung der Museumsbesucher angewiesen. Nur wenn sich die Besucher durch den Raum bewegen, können sie die Exponate zu Gesicht bekommen, auf denen - wie wir sehen werden - die Wissensvermittlung in der Ausstellung basiert (vgl. Zebhauser 2000 zur Rede vom Museum als ,begehbarem Medium'). Auch hängt von ihren Bewegungsentscheidungen ab, welche Objekte sie sehen, wie viele und welche Details sie an den einzelnen Objekten erkennen können, in welcher Reihenfolge und Kombination (Gesichtsfeld) sie die präsentierten Objekte wahrnehmen usw. ${ }^{8}$ Daher sind die Bewegungen der Besucher durch den Ausstellungsraum ein wichtiger Aspekt der sozialen Praxis des gemeinsamen Museumsbesuchs. Wenn man diese soziale Praxis beschreiben will, muss man die spezifischen Formen der Bewegung durch den Ausstellungsraum rekonstruieren. Das soll in diesem Kapitel geschehen.

Wir werden sehen, wie die Besucher durch ihre Bewegungen, die sie aufeinander abstimmen („Ko-Orientierung“) einen gemeinsamen Bewegungsraum herstellen, einen der drei Teilräume, die zusammen ihren Interaktionsraum bilden.

7 S. etwa Hindmarsh/Heath (2003), vom Lehn/Heath/Hindmarsh (2001) oder vom Lehn/Heath (2007b).

8 Die Bedeutsamkeit der Besucherbewegung findet ihren Niederschlag in der großen Zahl von Formen, die im Ausstellungsraum die Aufgabe der Organisation von Bewegung und Wahrnehmung bearbeiten und die wir in 3.3 untersucht haben. 
Und wir werden sehen, dass sie diese Bewegungen auf eine ganz spezifische, für die soziale Praxis des gemeinsamen Museumsbesuchs musterhafte Weise realisieren. Die Bewegungen der Museumsbesucher und der aus diesen Bewegungen entstehende Bewegungsraum sind derart charakteristisch, dass sie für kompetente Beobachter als Bewegungen beim gemeinsamen Museumsbesuch wiedererkennbar sind. Sie wären es wahrscheinlich selbst dann noch, wenn man aus den Videoaufnahmen Ausstellungsraum und Exponate wegretuschieren würde. In diesem Sinne kann man sagen, dass die Bewegungen der Besucher ihre räumliche Umwelt als Ausstellungsraum ausweisen oder, konversationsanalytisch gesprochen, dass sie ihn in ihrer Interaktion als Ausstellungsraum hervorbringen.

Bei den Bewegungen der Besucher durch den Ausstellungsraum spielt die Bezugnahme auf die räumliche Umwelt eine zentrale Rolle. Ein Gutteil der Spezifik der Bewegungen durch den Ausstellungsraum ergibt sich aus der Bezugnahme auf das, was im Ausstellungsraum lange schon vorhanden ist, wenn ihr Besuch beginnt. Die Bewegungen der Besucher tragen also nicht nur dazu bei, die räumliche Umwelt als Ausstellungsraum zu konstruieren, die Bewegungen orientieren sich auch an dem, was die Besucher im Ausstellungsraum vorfinden und erhalten von diesem Verstehenshintergrund ihren spezifischen Sinn: So kann eine Bewegung durch die Bezugnahme auf das in der räumlichen Umgebung Vorgefundene z.B. zu einer „Annäherung an ein Exponat“ werden. ${ }^{9}$

Was ist also das Charakteristische der Bewegungen der Besucher beim gemeinsamen Museumsbesuch und was charakterisiert den museumsspezifischen Zuschnitt des Bewegungsraums? Folgende Aspekte sollen im Folgenden datenbasiert herausgearbeitet werden:

- Wie bringen die Besucher ihre Bewegungen als gemeinsame hervor, wie machen sie sichtbar, dass sie nicht zufällig mit anderen im Ausstellungsraum anwesend sind, sondern dass sie mit diesen zusammen das Museum besuchen? Diesen Aspekt des Museumsbesuchs haben speziell vom Lehn, Heath und Kollegen in den Mittelpunkt ihrer Forschungen gestellt (s. etwa vom Lehn/Heath/Hindmarsh 2001, vom Lehn 2013), wichtige Anknüpfungspunkte gibt es aber auch zu Arbeiten, die sich speziell dem gemeinsamen Gehen (Ryave/Schenkein 1974, Schmitt 2012) oder allgemeiner mit der Organisation mobiler Interaktion beschäftigen (McIlvenny/Broth/Haddington

9 Wenn hier immer von Bewegungen die Rede war, heißt es nicht, dass andere Modi - speziell der Modus Sprache - in der Analyse ignoriert werden könnten. Wir werden sehen, dass auch für die Rekonstruktion des musealen Bewegungsraums ein Augenmerk auf Sprache unverzichtbar ist. 
2009, Haddington/Mondada/Nevile 2013 oder Stefani et al. 2019). ${ }^{10}$ Dieser Aspekt wird in 5.2.2 behandelt.

- Welche Rolle spielen die Exponate für die Bewegungen der Besucher durch den Ausstellungsraum? Diese Frage, der sich Abschnitt 5.2.3 widmen wird, hat bisher vor allem die empirische Besucherforschung interessiert. Untersucht wurde, wie bestimmte Merkmale des Raums die Bewegung durch die Ausstellung allgemein und speziell die Wahl von Bewegungszielen beeinflussen (z.B. Bitgood 2006, mit Literaturüberblick), oder als Maß für die Attraktivität oder ,holding power' eines Exponats (kritisch zu diesen Studien: Heath/vom Lehn 2001: 289 oder vom Lehn/Heath/Hindmarsh 2001: 197). Speziell zu Formen der Bewegung bei der Rezeption einer Kunstinstallation s. Winiger (im Druck).

- Wie erzeugen die Besucher die übergeordnete Struktur des ,Rundgangs‘? Diese Frage soll in 5.2.4 beantwortet werden. Sie wurde bisher vor allen unter praktischen Gesichtspunkten untersucht, also unter dem Gesichtspunkt, wie die Bewegungen der Besucher gesteuert werden können (s. z.B. Dean 1994). Theoretisch fundiert untersucht vor allem die Space Syntax den Zusammenhang zwischen der Anlage der Ausstellungsräume und den Bewegungen der Besucher (s. Choi 1999, Peponis et al. 2004, Wineman/Peponis 2009, Kaynar Rohloff 2009), und Véron/Levasseur (1991) erforschen aus ethnographischer Perspektive unterschiedliche Muster von Besucherbewegungen durch den Ausstellungsraum. Dabei verknüpfen sie die Konstruktion des Rundgangs durch die Körper der Besucher mit der Konstruktion der Ausstellungsbedeutung:

Le vrai discours de l'exposition est celui que construit le visiteur par son parcours, en mettant en relation dans un certain ordre ce qui lui est proposé. La signification n'est pas donnée à l'entrée, elle est produite à la sortie. (Véron/Levasseur 1991: 18)

- Welche Rolle spielt schließlich der gemessene Gang für die Musterhaftigkeit der Bewegungen durch den Ausstellungsraum? Hierzu gibt es vor allem impressionistische Aussagen, die auf die geringe Geschwindigkeit (vgl. Treinen 1981: 25) und die zur Schau gestellte Würde des Gehens im Museum abheben (Klingenstein 2001, Rumpf 1995: 30, Brock 2000: 454). Für das Verständnis des Zusammenhangs zwischen einer bestimmten Art und Weise der Bewegung und der Kategorisierung als Besucher bietet Lee/Watson (1992-

10 Entferntere Anknüpfungspunkte bieten: Certeau (1988) zum Gehen als Mittel der Raumkonstitution; Linke (2010) zu Bewegungen des Körpers als einem der Aspekte des „kommunikativen Auftritts“; Tschofen (2013) zum Gehen aus kulturwissenschaftlicher Perspektive mit einigen Anmerkungen zum Gehen im Museum (2013: 71-73); Pink (2007) zum ,Gehen mit Video' als anthropologischer Erhebungsmethode. 
1993) einen interessanten Anknüpfungspunkt. Die Autoren zeigen nämlich, wie sichtbares Verhalten im öffentlichen Raum und soziale Kategorisierungen miteinander verbunden sind. Die Rekonstruktion der spezifischen Art der Bewegungen beim gemeinsamen Museumsbesuch ist Gegenstand von 5.2.5.

\subsubsection{Die Ko-Orientierung der Bewegungen im Ausstellungsraum}

Ich möchte die Analyse des musealen Bewegungsraums mit der Untersuchung eines längeren Einzelfalls beginnen. Der gewählte Videoausschnitt zeigt einen Vater mit seiner etwa fünfjährigen Tochter „Nina“ (NI) beim Besuch des Zoologischen Museums. Ein jüngeres Geschwisterkind schläft während des gesamten Ausschnitts im Kinderwagen. Mit Einsetzen des Transkripts ,lockt' Nina ihren Vater „Patrick“ (PA) zu einem großen, freistehenden Mammutexponat, das beide längere Zeit betrachten, bevor sie dann zu einem Riesenhirsch-Exponat weitergehen.

An diesem Beispiel möchte ich auf einen Aspekt der Bewegungen durch den Ausstellungsraum hinweisen, der kaum je in den Blick der musealen Besucherforschung geraten ist: die Tatsache, dass die Bewegungen der Personen, die gemeinsam ein Museum besuchen, eng aufeinander abgestimmt sind. ${ }^{11}$

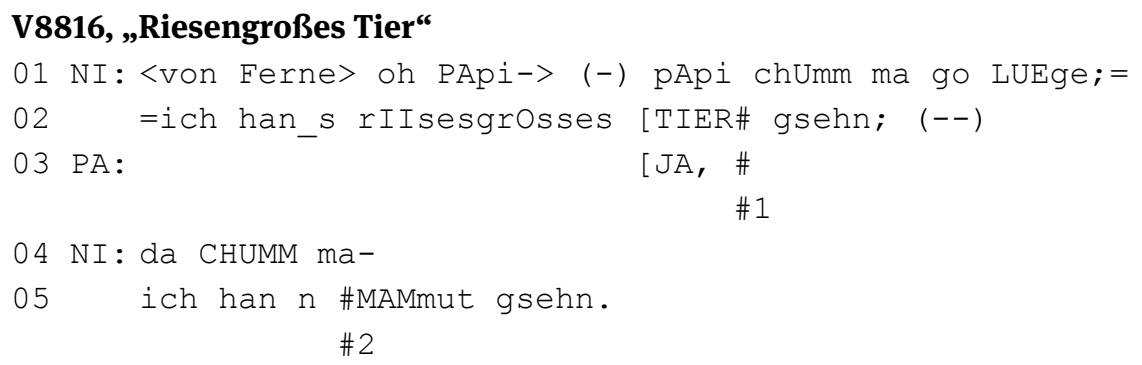

11 Diese Vernachlässigung ist um so erstaunlicher, als von Museumsarchitekten und Ausstellungsgestaltern viel dafür getan wird, Möglichkeiten für ein gemeinsames Betrachten der Ausstellungsstücke zu schaffen (oder ein gemeinsames Bedienen der Exponate: Heath/vom Lehn/ Osborne 2005). 

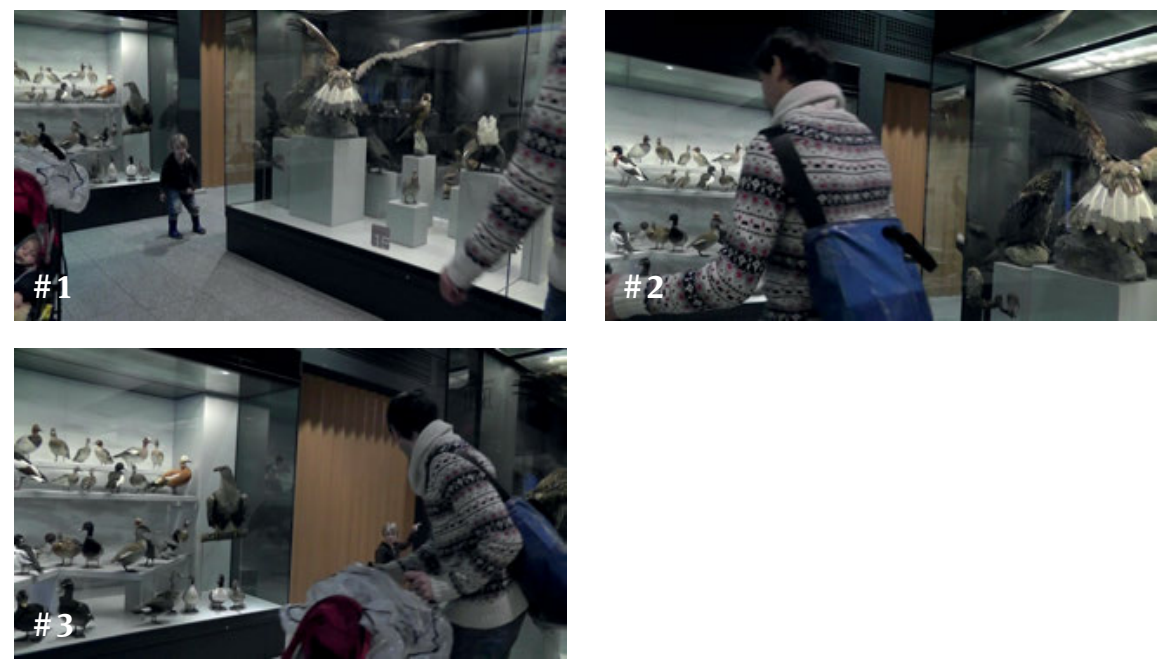

$\begin{array}{ll}06 & \text { n } \\ 07 & \text { M } \\ 0 & \text { MAMm }[\text { mut. }\end{array}$

08 PA: \#[en MAMmut;

\#3

$09 \quad(2.0)$

10 NI: \#((klatscht in die Hände) \#((klatscht, 3 sek.)) \# 4 \# 5
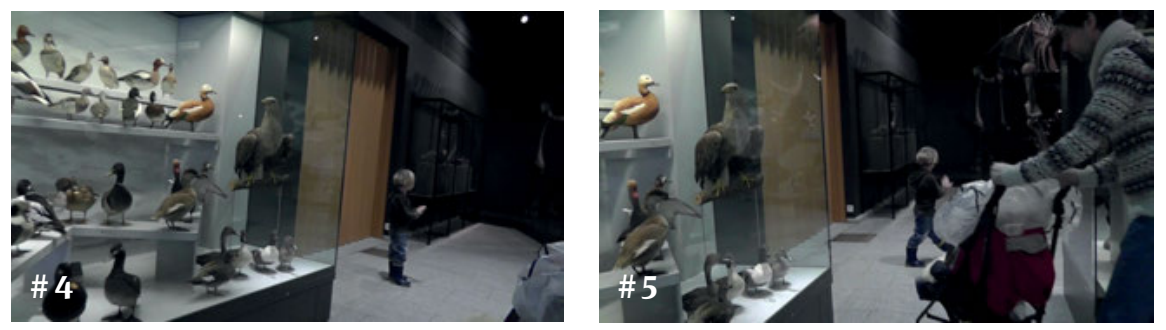

$11 \mathrm{PA}: \# \uparrow{ }^{\circ} \mathrm{OH}:: \mathrm{ja} \cdot(4.1) \#(0.3) \#(0.5) \#$

\# 6 \#7

\# 8 \# 9
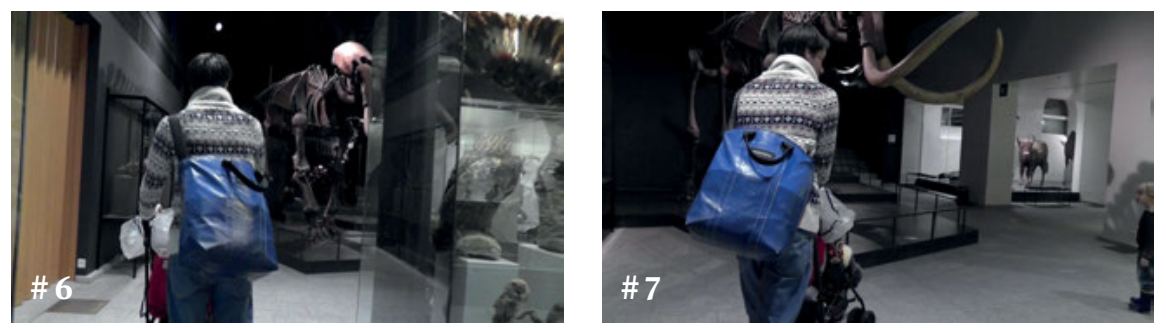

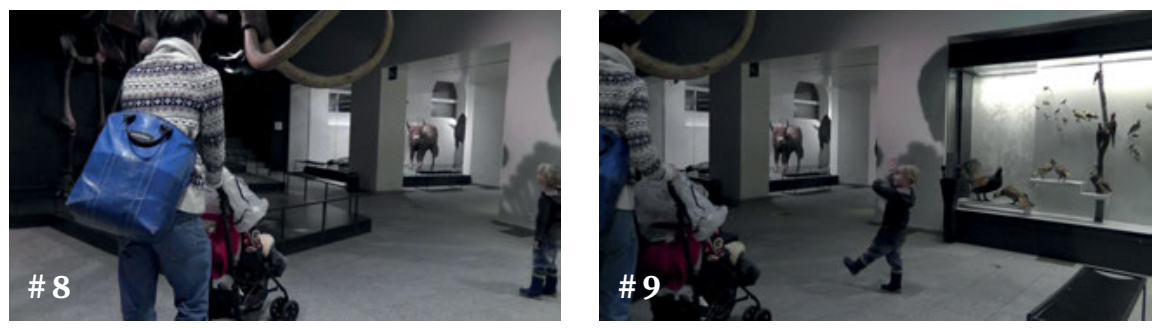

12 NI: $\langle<p>$ (chammer) $>$ sich grAd dra HEbe.\#

\#10

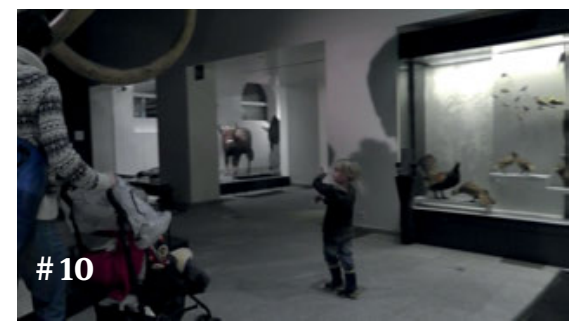

In dieser kurzen Phase des Museumsbesuchs von Nina und Patrick können wir bereits eine große Zahl von Aktivitäten beobachten, mit denen die beiden Besucher die Aufgabe der „Ko-Orientierung“ (Hausendorf 2010) bearbeiten, so dass ihr Gehen durch den Ausstellungsraum als ein gemeinsames Gehen, ein „Miteinander Gehen“ (Schmitt 2012) zu erkennen ist:

- Nina, die am Ende des Vitrinengangs nach rechts abgebogen ist und dort ein Mammut entdeckt hat, kehrt um und fordert ihren Vater auf, ihr zu folgen (,chumm“, Z. 1).

- Patrick beginnt sofort, auf den abgestellten Kinderwagen zuzugehen. Nina rennt schnell los, bleibt aber schon nach wenigen Schritten an einer Stelle stehen, von der aus sie ihren Vater noch sehen kann. Von dort aus winkt sie ihren Vater heran (man sieht Ninas Hand, mit der sie den Vater heranwinkt, durch das Vitrinenglas, \#2).

- Der Vater wendet den Kinderwagen in Ninas Richtung, während Nina ihm mit einer deiktischen Geste die Richtung anzeigt, in der er das Mammut zu suchen hat (\#3).

- Nina geht erst dann weiter auf das Mammut zu, als ihr Vater begonnen hat, den Kinderwagen in die von ihr angezeigte Richtung zu schieben, womit deutlich wird, dass er seinen ursprünglichen Standort dauerhaft aufgeben wird (\#5). 
- Nina geht nun zum Mammutexponat und bleibt dort stehen. Dabei wählt sie eine Position, die es ihr erlaubt, Patricks Näherkommen zu überwachen (\#6). Patrick schiebt den Kinderwagen auf Nina zu und bringt ihn in ihrer Nähe zum Stehen (\#9).

Mit anderen Worten, die beiden Besucher tragen dafür Sorge, dass ihre Bewegungen durch den Raum ebenso wie ihr Stehenbleiben fein aufeinander abgestimmt sind: Nina geht nicht direkt zum Mammutexponat, sondern nimmt mehrere aufeinander folgende Positionen ein, die es ihr erlauben, Patricks Annäherung an das Exponat kleinschrittig zu überwachen und zu steuern, und Patrick orientiert sich mit seinen Bewegungen im Raum an dem, was Nina ihm anzeigt. Diese gegenseitige Ko-Orientierung der Bewegungen erlaubt es, Patrick und Nina als Goffman'sches With zu sehen, also als Personen, die in der Wahrnehmungssituation gemeinsam präsent sind und gemeinsam etwas tun. Während bei anderen Typen fokussierter Interaktion wie beispielsweise einem Tennis- oder mehr noch: einem Cricketspiel zwischen den Beteiligten ein großer Abstand vorhanden sein kann, bringen die Koorientierungsaktivitäten beim gemeinsamen Museumsbesuch eine charakteristische räumliche Nähe der Interaktionsbeteiligten hervor (vgl. Ryave/Schenkein 1974: zu „spatial proximity“), die man auf den folgenden exemplarischen Abbildungen erkennen kann.
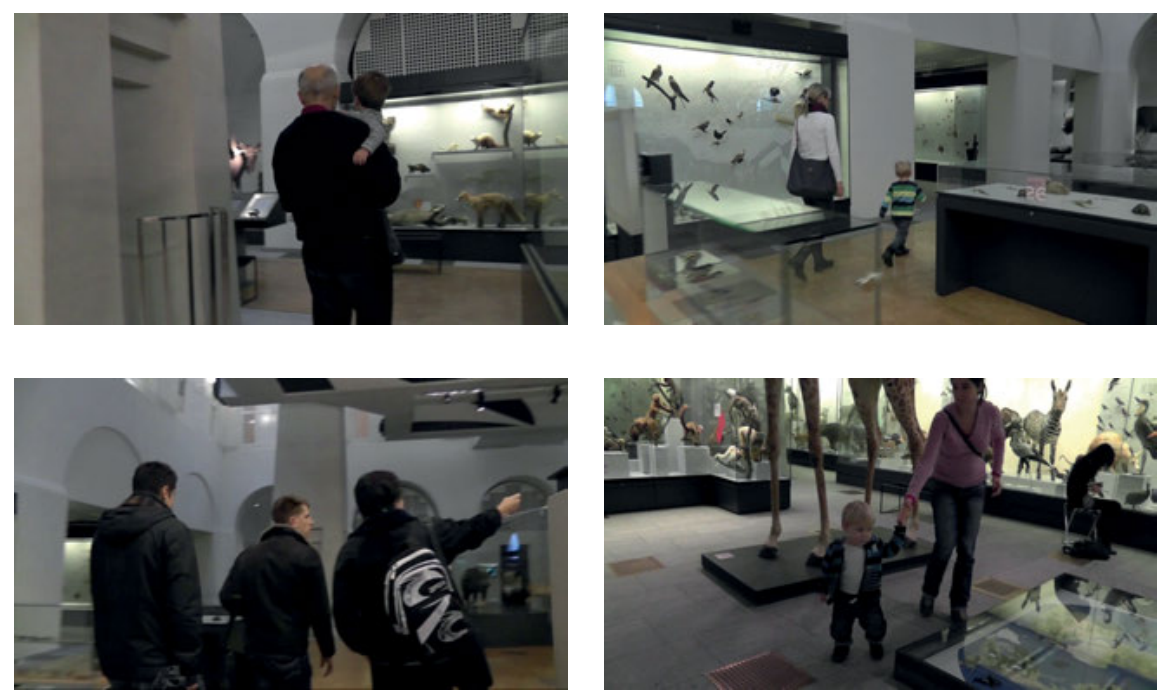

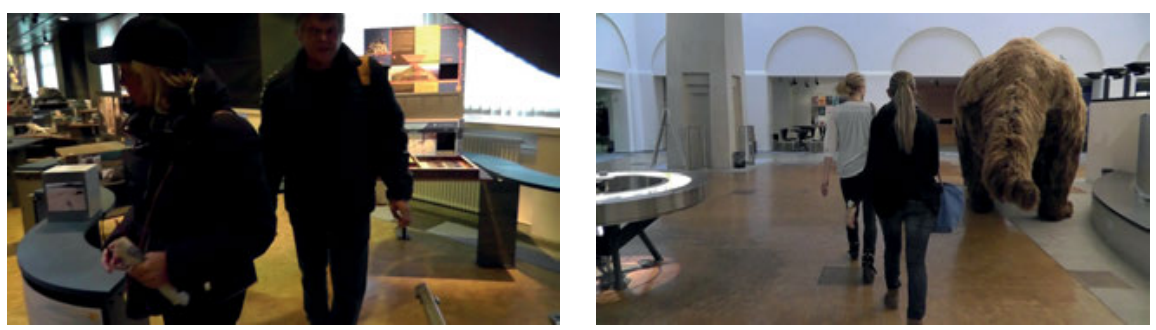

Abb. 5-1 - 5-6: Standbilder aus verschiedenen Videos

Dass die Interaktionsbeteiligten ihre Bewegungen im Sinne einer Ko-Orientierung aufeinander abstimmen, ist nicht nur beim gemeinsamen Museumsbesuch der Fall. Vielmehr lassen sich derartige Aktivitäten der Ko-Orientierung in allen Formen fokussierter Interaktion nachweisen (,focused gatherings“, Goffman 1963). Um zu beschreiben, was die Bewegungen im Rahmen der sozialen Praxis des gemeinsamen Museumsbesuchs wiedererkennbar macht, müssen also weitere Charakteristika der Besucherbewegungen rekonstruiert werden.

\subsubsection{Die Rolle der Exponate für die Bewegungen im Ausstellungsraum}

Einem zentralen Aspekt der Besucherbewegung möchte ich im folgenden Abschnitt nachgehen. Es handelt sich um die Rolle, die die Exponate für die Organisation der Besucherbewegungen spielen: und zwar als Motiv und Ziel von Bewegungen durch den Ausstellungsraum sowie als Kern einer ,Verweilzone‘, in der die Besucherbewegung zu einem längeren Stillstand kommt.

Schauen wir uns dazu erneut den Transkriptausschnitt „Riesengroßes Tier“ an, jetzt allerdings unter der Fragestellung, wie Besucher ihre Bewegungen durch den Raum als Annäherung an ein Ausstellungsstück erkennbar werden lassen. Schon in Ninas erster Äußerung - „oh PApi- (-) pApi chUmm ma go LUEge; \&ich han_s rIIsesgrOsses TIER gsehn;“(Z. 1) - wird diese Orientierung deutlich. Mit ihrer Äußerung versucht Nina, ihre Entdeckung als neues gemeinsames Bewegungsziel im Ausstellungsraum zu etablieren. Hinweise auf diese Funktion geben

- der Imperativ, mit dem sie ihren Vater auffordert, ihr zu folgen (,chumm ma go luege“),

- die Emphase, die in ihrer Wortwahl (,rIIsesgrOsses“), der einleitenden Interjektion („oh“) und in den rhythmischen Betonungen zutagetritt,

- ihre dynamische Körperhaltung, der man ansieht, dass sie ,auf dem Sprung ist und gleich loslaufen wird (vgl \#2), und

- das mehrfache Vorkommen von Verben des Sehens („LUEge“, „gsehn“). 
Dass es sich bei dem von Nina angesteuerten Ziel um ein Exponat handelt, wird in Ninas Reformulierung ihrer Entdeckung deutlich: „ich han n MAMmut gsehn.“. Denn bei Mammut handelt es sich um eine Kategorie aus dem Kategorienset zoologische Gattungen, und mit genau diesem Kategorienset wird in dem hier untersuchten Museum auf die Exponate referiert (neben Sets, die höhere oder niedrigere Stufen innerhalb der zoologischen Taxonomie betreffen, s.o. 3.6). Der Vater signalisiert daraufhin, dass er das vorgeschlagene Bewegungsziel akzeptiert, indem er - wie oben beschrieben - auf den abgestellten Kinderwagen zugeht und beginnt, ihn ,umzurangieren“ (\#2) und indem er wiederholt: „en MAMmut;“.

Die anschließende Bewegung durch den Ausstellungsraum ist damit von Vater und Tochter als Bewegung hin zu einem Exponat, hier: dem Mammutexponat, definiert worden. Wie zeigen sich Patrick und Nina nun ihre Ausrichtung auf das Exponat an?

- In \#2 zeigt Nina den Standort ihrer Entdeckung mit ihrer Zeigegeste an. Gleichzeitig ist ihr Oberkörper nicht auf ihren Vater ausgerichtet, sondern ,vermittelt' zwischen der Position des Vaters und der des Exponats.

- Während Nina auf ihren Vater wartet, richtet sie ihren Kopf auf das Exponat aus (\#3). Diese Ausrichtung auf das Exponat behält sie auch dann noch bei, als sie vorwärts zu laufen beginnt (\#4). Das suggeriert, dass es für sie in dieser Richtung etwas zu sehen gibt, das ihr wichtiger ist, als die Wahrnehmung etwaiger Hindernisse im Laufweg.

- Zum Zeitpunkt, den \#5 dokumentiert, hat Patrick einen Standpunkt erreicht, von dem aus er das Mammut sehen kann. Er schaut in die von Ninas Haltung angezeigte Richtung und bestätigt mit „OH:: ja.“ (Z. 11) zum einen die von Nina behauptete Berechtigung ihrer Aufregung, die ich oben als Hinweis auf das Mammut als Gegenstand des Staunens gedeutet habe (Betonung, hoher Sprung zum Gipfel der Akzentsilbe und tiefer Fall der Intonationskurve am Ende), zum anderen die Richtigkeit der Identifizierung des Exponats als Mammut (,ja“).

- In \#6 sieht man Nina, mit dem Unterkörper auf das Mammut ausgerichtet, den Kopf aber in Patricks Richtung gewendet. So markiert sie die Stelle, der Patricks Interesse gelten soll.

- Mit Patricks Näherkommen wendet Nina ihren Blick dem Mammut zu (\#7). Der Vater übernimmt Ninas Relevantsetzung des Mammutexponats: Auch er dreht den Kopf aus seiner Bewegungsachse heraus, während er sich weiter der Position seiner Tochter nähert, und richtet ihn auf das Mammut, anstatt den Raum vor seinen Füßen zu kontrollieren (\#8).

- Dann bringt er den Kinderwagen zum Stehen und markiert so das Ende der großräumigen Bewegung durch den Ausstellungsraum (\#9). Jetzt haben beide Besucher eine Position erreicht, von der aus sie sich dem Mammut widmen 
können, konkret: es im Detail betrachten (s.u. 5.3) und es zum Gegenstand ihrer interaktiven Wissenskonstruktion machen können (s.u. 5.4).

Bisher sind in der Regel nur die letzten Sekunden der Annäherung an ein Exponat untersucht worden (s. etwa Heath/vom Lehn 2001). Der Ausschnitt „Riesengroßes Tier“ zeigt nun, dass die Exponate für die Bewegungen der Museumsbesucher schon wesentlich früher relevant sind, als bisher gezeigt worden ist. Für alle Bewegungen durch den Ausstellungsraum stellen die Exponate eine wichtige Orientierungsgröße für die Besucher dar. Die Exponate treten als Motiv und Ziel der Bewegungen durch den Raum in Erscheinung: Die Besucher strukturieren den Ausstellungsraum, indem sie ihre Bewegungen als Bewegungen zu Exponaten definieren. Hierin scheint mir ein wichtiges Element der Musterhaftigkeit des musealen Bewegungsraums zu liegen.

Schon in diesem Ausschnitt sieht man, dass die Bewegungen der Besucher nicht einfach aus Eigenschaften der Exponate resultieren. Sie sind - wie auch vom Lehn/Heath/Hindmarsh (2001) betonen - das Ergebnis von Aushandlungsaktivitäten der Besucher. Im nächsten Ausschnitt („Läck!“) wird das meines Erachtens klarer.

\section{V8816, „Läck!“ \\ 01 NI: \# $(\mathrm{xxx})$ \#L L̈̈CK- \#1 \#2}
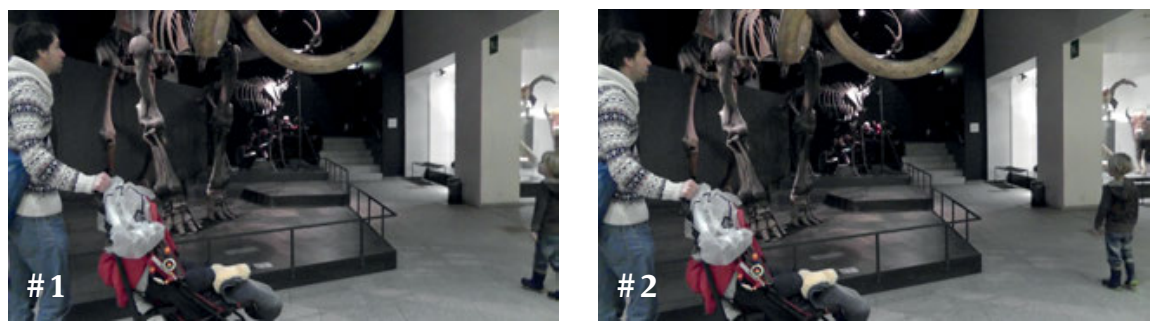

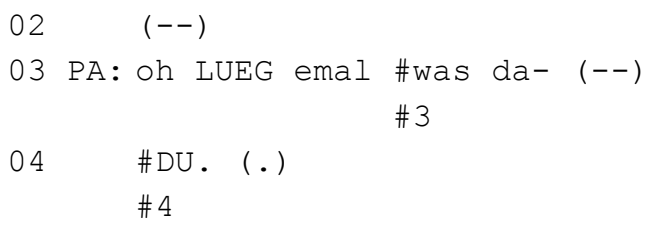

$02 \quad(--)$

03 PA: oh LUEG emal \#was da- (--)

\#3

\#4 


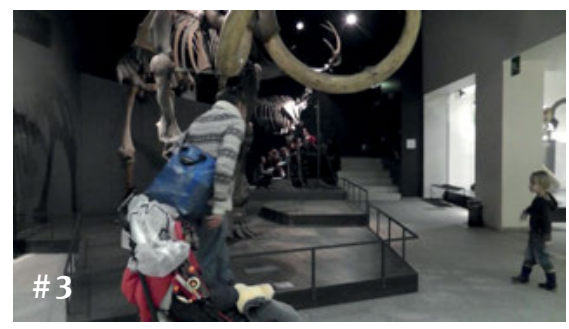

05 LUEG emal.\# (---)

$$
\text { \# } 5
$$

06 das isch n MAMmut.\#

\#6

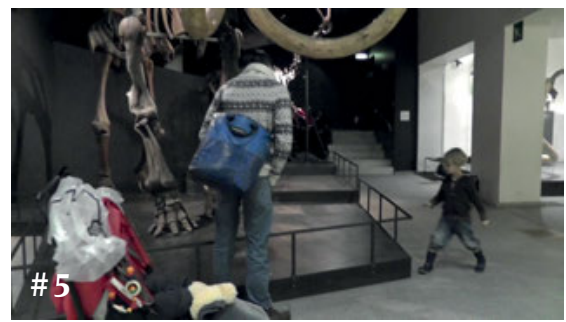

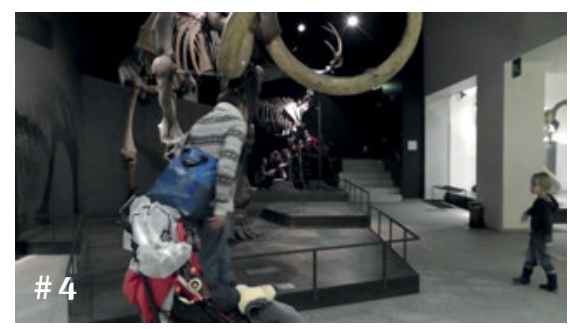

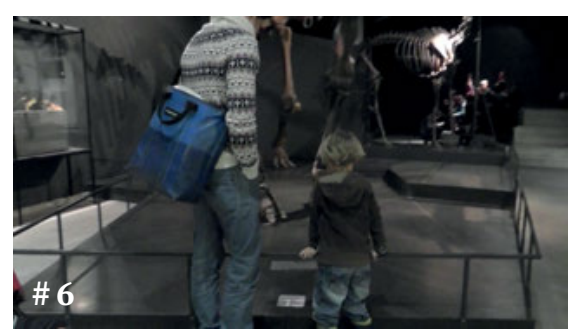

In diesem kurzen Ausschnitt stehen zwei alternative Bewegungsziele zur Debatte.

- Nina, die mit dem Kopf im Nacken die Stoßzähne des Mammuts betrachtet hat (\#1), senkt abrupt den Kopf und geht gleichzeitig einen Schritt nach vorne (\#2). Diese Kopf- und Körperbewegung stellt eine ,Einladung zur Abwendung' dar, wie sie vom Lehn (2013) untersucht hat. Mit ihrem emphatischen Läck! (Z. 1) signalisiert sie, dass sie etwas Überraschendes entdeckt hat. - Man kann vermuten, dass sich dieses Überraschende in ihrer Blickund Bewegungsrichtung befindet.

- Patrick geht allerdings nicht auf diese Initiative ein. Er nähert sich vielmehr einer Stelle, an der sich ein Objekttext zum Mammutexponat befindet, und signalisiert Nina, dass er an dem aktuell betrachteten Exponat etwas Sehenswertes entdeckt hat: „oh LUEG emal was da-“ (Z. 3). Patrick lehnt also nicht nur Ninas Initiative ab, er setzt ihr eine eigene ,Bewegungseinladung‘ entgegen.

- Nina verfolgt ihre Initiative weiter, indem sie ihr Zeigen in den Raumhintergrund fortsetzt und einen Schritt in die angezeigte Richtung geht (\#3). Erst nach der direkten Anrede durch den Vater („DU.“ , Z. 4) schaut sie zu diesem hin, lässt ihren immer noch zeigenden Arm sinken (\#4) und beginnt, sich der Position des Vaters zu nähern, bis sie schließlich Seite an Seite neben ihm zu stehen kommt (\#5). 
Die Bewegungen durch den Ausstellungsraum sind hier von Patrick und Nina interaktiv ausgehandelt worden. Ninas ,Einladung zur Abwendung“ (als „first action“ einer Paarsequenz, s. erneut vom Lehn 2013) hat allerdings nicht Patricks Abwendung vom Mammutexponat zur Folge. Seine Schritte auf den Objekttext zu können als Ablehnung von Ninas Einladung interpretiert werden (als „second action“). Gleichzeitig dienen sie als eine Art Gegenvorschlag, der von Nina mit einer Bewegung hin zu der vom Vater markierten Stelle im Raum akzeptiert wird.

Die Ausschnitte „Riesengroßes Tier“ und „Läck!“ haben nicht nur deutlich werden lassen, dass die Bewegungen während des Museumsbesuchs interaktiv ausgehandelt werden und dass die Exponate als Ziele für die Bewegungen durch den Ausstellungsraum eine zentrale Rolle spielen. Sie geben auch einen Eindruck davon, was die Besucher in die Waagschale werfen können, um die Bewegung hin zu einem Exponat hervorzubringen oder um auszuhandeln, ob man sich von dem Exponat abwenden soll.

Bezieht man weitere Fälle aus meinem Korpus in die Analyse ein, zeigt sich, dass sich die Bewegungsrelevanz der Exponate bzw. der Vitrinen, die die Exponate enthalten, grundsätzlich aus zwei Quellen speist. Zum einen aus dem Charakter der Exponate als Gegenstand der Anschauung. So reicht es im Museumskontext bisweilen aus, das Vorhandensein von etwas Sichtbarem $\mathrm{zu}$ behaupten oder ein unspezifisches Sehen einzufordern (,Schau mal da!'), um eine gemeinsame Bewegung hervorzubringen. Zum anderen kann sich die Bewegungsrelevanz der Exponate aus ihrem Charakter als Wissensobjekt ergeben: Die Bewegung zum Exponat verspricht die Beantwortung von thematisch einschlägigen Fragen (die häufig implizit bleiben: [Was ist das für ein Exponat?] - „das isch n MAMmut.“). Häufig kommt - wie in beiden bisher untersuchten Ausschnitten - die Darstellung einer starken emotionalen Beteiligung hinzu, die das zu Sehende oder zu Lernende als etwas Aufregendes, Spannendes, Staunen oder auch Bewunderung und Ehrfurcht Hervorrufendes kennzeichnet. Die Abwendung von einem Exponat bzw. einer Vitrine lässt sich durch Verweis auf die gleichen Aspekte des Exponats durchsetzen, lediglich mit umgekehrtem Vorzeichen: nämlich indem man anzweifelt, dass man an dem Exponat etwas sehen (,ich glaub da gseht gseht mer z_WEnig“, „Sybille“ in V1358) oder lernen kann (,s tierquiz das is für GRÖSsere.“, „Alexandra“ in V1221).

Die Relevanz der Exponate für die Bewegung der Besucher durch den Ausstellungsraum ergibt sich aus ihrer Bedeutung für den musealen Wahrnehmungsund Handlungsraum, die ich in 5.3 und 5.4 untersuchen werde: ihre Bedeutung als Zentrum eines gemeinsam hergestellten Raumes geteilter Aufmerksamkeit und als Kristallisationspunkte für die Wissenskommunikation im Ausstellungsraum. Das ist auch daran zu sehen, dass es streng genommen nicht die Exponate 
selbst sind, die das Ziel der Bewegungen durch den Ausstellungsraum darstellen, auch wenn dies von den Besuchern so formuliert wird (z.B. „gömmer zum FUULtier;“, „Stephanie“ in V4647). Tatsächlich ist es der Raum unmittelbar vor den Exponaten, der den Endpunkt der Besucherbewegungen durch den Ausstellungsraum bildet und in dem sich die stationären Phasen des Museumsbesuchs abspielen. Dieser Raumbereich, den ich in 3.3.2 „Verweilzone“ genannt habe (im Gegensatz zu den „Gehzonen“, die die Besucher in den mobilen Phasen ihres Museumsbesuchs nutzen), wird durch die Möglichkeiten und Beschränkungen strukturiert, die er für die Herstellung eines gemeinsamen Betrachtungs- und Handlungsraums bietet.

Da ich die Herstellung dieser beiden Teilräume weiter unten im Detail analysieren werde, möchte ich hier nur kurz auf zwei Beobachtungen in den obigen Ausschnitten hinweisen, die diese Strukturierung der Verweilzone illustrieren können. Auf Standbild \#9 aus dem Ausschnitt „Riesengroßes Tier“ (s.o., S. 325) sehen wir Patrick und seine Tochter Nina zu einem Zeitpunkt, an dem ihre Annäherung an das Mammut zum Stillstand gekommen ist. Beide stehen in einigem Abstand zum Mammut, genauer: in einer Entfernung und mit einer Orientierung, die es beiden erlaubt, das Exponat als Ganzes zu betrachten, speziell aber die Stoßzähne, um die es in diesem Gesprächsabschnitt geht. Diese gemeinsame Orientierung ist auch dafür verantwortlich, dass man Patrick und Nina als Personen wahrnimmt, die sich gemeinsam im Ausstellungsraum aufhalten, selbst wenn die Entfernung zwischen ihnen im Vergleich zu anderen Daten aus meinem Korpus beträchtlich ist. Die Verweilzone am Endpunkt der Bewegung durch den Ausstellungsraum befindet sich also an einer Stelle, von der aus das angesteuerte Exponat gemeinsam als Objekt der Betrachtung und der Wissenskonstruktion genutzt werden kann.

Auf Standbild \#6 aus dem Ausschnitt „Läck!“ (s.o., S. 330) stehen Patrick und Nina deutlich näher beieinander. Und sie befinden sich so nah am Exponat, wie es das kleine Geländer um das Mammutpodest gestattet. Hier lässt sich ihre Position vor dem Exponat mit Bezug auf den Objekttext verstehen, der am Boden angebracht ist und den sie nutzen, um Informationen zum Mammut zu erhalten (,das isch n MAMmut.“). Die Position von Vater und Tochter ergibt sich also aus der Nutzung des Exponats als Wissensobjekt. In diesem zweiten Fall sieht man, besser noch als in „Riesengroßes Tier“, die räumliche Formation, die Besucher charakteristischerweise am Ende einer Bewegung durch den Ausstellungsraum einnehmen: Eine „I-formation“ (Ciolek/Kendon 1980: 249), in der die Besucher Seite an Seite in unmittelbarer Nähe des Bewegungsziels stehen, die Körperfronten auf dieses Ziel hin ausgerichtet und einander nur von der Seite sehend.

Die Exponate haben aber auch Implikationen für die (angemessenen) Bewegungen im Rahmen des gemeinsamen Museumsbesuchs, die sich nicht aus 
ihrer Rolle für den musealen Wahrnehmungs- und Handlungsraum ergeben. So gehört zur Beschreibung der Bewegungen im Ausstellungsraum, die Teil der hier beschriebenen sozialen Praxis sind, dass die Exponate von Berührungen auszunehmen sind. Gerade bei Museumsbesuchen mit kleinen Kindern, die in meinem Korpus häufig vertreten sind, findet man häufig implizite sowie explizite Bezugnahmen auf diesen Bewegungsaspekt.

Der folgende Ausschnitt zeigt beispielhaft einen Fall, in dem das angemessene Bewegungsverhalten gegenüber Exponaten explizit behandelt wird. Es handelt sich um einen Ausschnitt aus dem Museumsbesuch von Patrick und Nina, der sich zeitlich genau zwischen den bisher untersuchten Ausschnitten befindet. Das Transkript setzt in dem Moment ein, als der Vater seine Begeisterung angesichts des Mammutexponats zum Ausdruck bringt.

\section{V8816, „Herumturnen“}
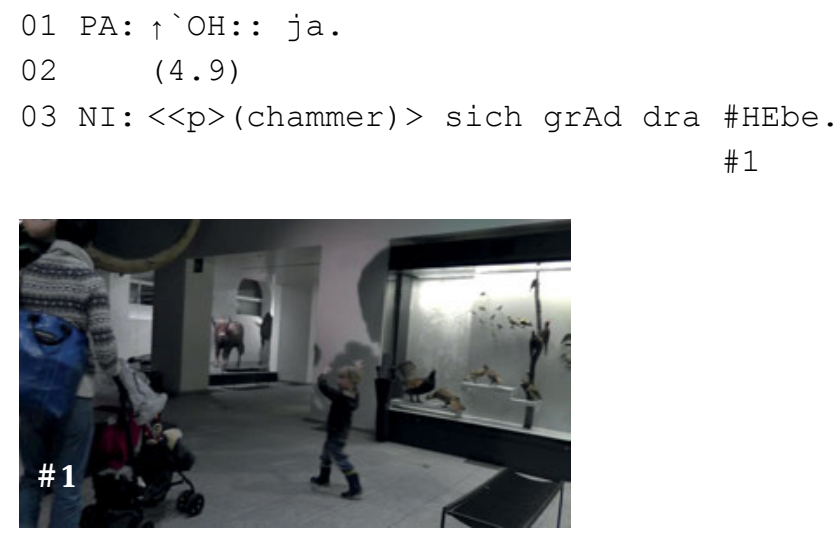

04 PA: ja das dÖrf mä NÖD,

05 abr (-) die sind eso gross dass mer CHÖNNT;

06 GÄLL?

$07 \mathrm{NI}:\langle\langle\mathrm{p}\rangle \mathrm{JA} ;>$

08 PA: chönnt mä richtig druf UMeturne.

$09 \quad(---)$

10 NI: wemm (.) wemmer WÜRkli fescht druf Umetrampled_dänn

11 gönt $s$ kaputt.

$12(--)$

13 PA: jetz wär wahrschindlich so- (-)

14 (dass_s/das) da würd kaPUTT gah wemmer jetz da z fescht

15 dra riisst. 
16 NI: m_HM,

$17(---)$

18 LU: (XXX) L L̈̈K-

In diesem Ausschnitt handeln die beiden Besucher aus, wie mit dem Mammut umzugehen ist.

- Nina spricht von der Möglichkeit, sich am Skelett festzuhalten, und demonstriert diese, indem sie die Arme nach oben hebt, als schwinge sie sich an den Stoßzähnen des Mammuts vor und zurück (\#1).

- Patrick bestätigt diese Möglichkeit (,ja“), führt aber sogleich ein Verbot an, das gegen diese Möglichkeit spricht: „,das dÖrf mä NÖD,“ (Z. 4). Das Indefinitpronomen $m \ddot{a}\left(\operatorname{man}^{\prime}\right)$ zeigt, dass es sich nicht um eine persönliche Präferenz von Patrick handelt, sondern um eine allgemeine Regel.

- Daraufhin geht er, in leicht belustigtem Ton, auf die von Nina behauptete Möglichkeit des Sich-Anhängens ein. Doch während das Schaukeln bei Nina beinahe als konkreter Vorschlag daherkommt (vgl. den Indikativ „chammer“, Z. 3, und ihre Vorführung des Schaukelns), verleiht der Vater dem Schaukeln den Status einer bloßen Vorstellung (Konjunktiv II: „CHÖNNT“, Z. 5).

- Nina folgt der Stoßrichtung von Patricks Beitrag und führt die Zerbrechlichkeit des Exponats als Grund dafür an, das Mammut nicht als Klettergerüst zu nutzen. Dabei spricht nun auch sie - wie die Modalpartikel „WÜRkli“ unterstreicht - über das ,Herumtrampeln“ lediglich als Idee (Z. 10).

- Der Vater bekräftigt das Zutreffen ihrer Begründung und Nina bestätigt seine Aussage ihrerseits mit einem bejahenden „m_HM,“(Z. 16).

So haben die beiden Besucher die Nutzung des Exponats als Klettergerüst, die ja durch das niedrige Geländer nicht wirklich verunmöglicht wird, gemeinsam in das Reich der Phantasie verbannt.

In zahlreichen anderen Stellen meines Korpus tritt das Berührungsverbot weniger explizit zutage. Etwa wenn die Berührung eines Exponats, das Schlagen an oder das Klettern auf eine Vitrine körperlich unterbunden wird. Besonders subtil zeigt sich das Berührungsverbot in speziellen Formen des Zeigens während des Museumsbesuchs:

- beispielsweise wenn Besucher eine Zeigegeste äußerst nahe an ein frei stehendes Exponat heranführen, aber ohne es zu berühren, obwohl kein Glas die Berührung physisch verhindert (V2780, Abb. 5-7);

- oder wenn Besucher ihre Zeigegeste äußerst nahe an das Exponat heranführen, die Geste aber mit dem kleinen Finger ausführen, was suggeriert, dass die Bewegung keinesfalls ein Ergreifen des Exponats zum Ziel hat (V2910, Abb. 5-8). 


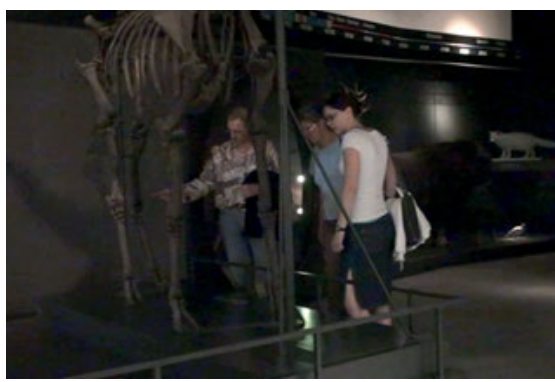

Abb. 5-7

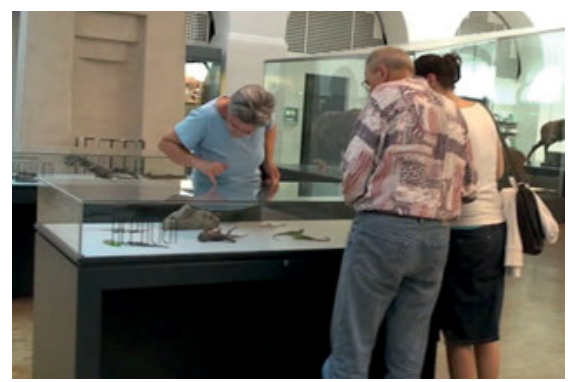

Abb. 5-8

Das Berührungsverbot zeigt sich nicht nur im beobachtbaren Verhalten der Besucher, es hat bisweilen auch im Raum Spuren hinterlassen. Etwa wenn man selbst dort, wo Exponate durch keinerlei physische Mittel vor einer Berührung geschützt sind, Schilder findet, die die Besucher auffordern, ein Exponat zu berühren (s.o. 3.4).

Im folgenden Abschnitt geht es um ein weiteres Charakteristikum der Bewegungen im Rahmen der sozialen Praxis des Museumsbesuchs: ihre Einordnung in einen übergeordneten ,Rundgang،.

\subsubsection{Der Rundgang}

Die Bewegungen durch das Museum werden von den Besuchern auf eine ganz spezifische Art und Weise organisiert: nämlich als eine Abfolge von Etappen, die von einem Exponat immer zu einem nächsten Exponat führen. Durch diese Organisation der Bewegung entsteht nicht nur das vielfach beschriebene Bummeln durch das Museum, das sich aus dem Wechsel von mobilen Phasen (Bewegung hin zum Exponat) und stationären Phasen (Verweilen vor dem Exponat) ergibt. ${ }^{12}$ Vielmehr entsteht auch eine spezielle räumlich-zeitliche Organisation des Museumsbesuchs, die - wie wir sehen werden - mit den Deiktika weiter und zurück ausgedrückt werden kann.

Im Folgenden werde ich einige Videoausschnitte daraufhin untersuchen, wie Besucher ihre Bewegungen als solch eine Reihe aufeinander folgender Betrachtungsziele organisieren.

Anhand des Ausschnitts „Tiger“ (s.a. Kesselheim 2016) möchte ich zeigen, wie Besucher den Museumsbesuch als Abfolge von aufeinander folgenden Ein-

12 Dieser Wechsel wurde auch bei Stadtführungen konstatiert (Stukenbrock/Birkner 2010, Kesselheim 2010b). 
zelbetrachtungen gestalten können und Abweichungen von der Ausrichtung am jeweils nächsten Exponat sowie Abweichungen von der strikten Sequenzierung der Betrachtungsvorgänge korrigieren. $\mathrm{Zu}$ sehen ist eine Gruppe von drei Personen, bestehend aus der französischsprachigen Mutter „Natalie“ (NA) mit ihrem kleinen Sohn „Tommy“ (TO) und ihrer deutschsprachigen Schwiegermutter „Mona“ (MO). Die Gruppe hat sich zuletzt eine Meeresspinne angeschaut, nun gehen sie eine Treppe hoch.

\section{V0300, „Tiger“ Teil 1}

$01 \mathrm{NA}$ : et ÇA C'est quOI tommy c'est QUOI, \#

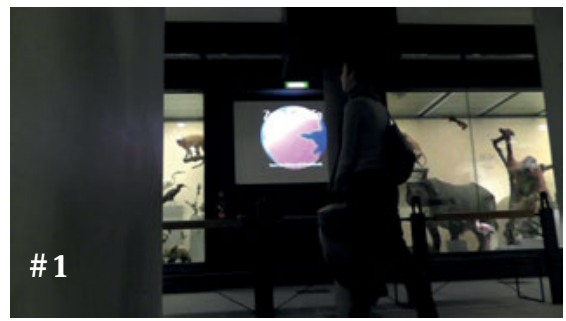

$02 \quad(-)$

03 hein ÇA,

$04 \quad(1.9)$

05 TOMmy?

$06 \mathrm{MO}:$ aHA jetz

$07 \mathrm{NA}$ : er geht (xxx) GANZ woandershin\# als wo man ihn jetzt $(-)$
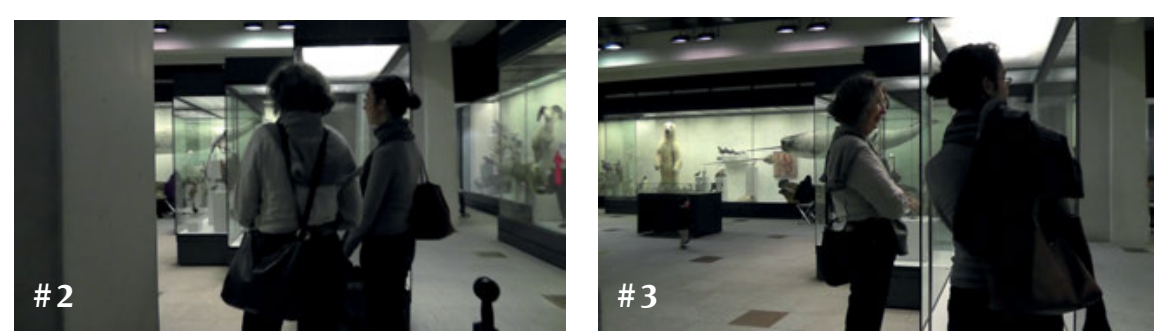

Die Treppe hat die Gruppe zu einer Reihe neuer potenzieller Bewegungsziele geführt. Natalie versucht, Tommy für eines dieser Ziele zu interessieren, indem 
sie auf ein Exponat zeigt und ihren Sohn auffordert, es zu benennen (\#1, Z.1). Tommy, der schon vorgegangen ist, geht nicht auf Natalies Initiative ein, sondern verfolgt seinen eigenen Weg, woraufhin seine Mutter ihre Initiative expandiert (Z.3-5). Als Tommy wieder nicht reagiert, schauen beide Frauen ihm zunächst hinterher (\#2). Dann kennzeichnen sie seine Bewegung als abweichend („lässt sich nIchts VORschreiben“, Z. 8) und als Verstoß gegen ihre eigenen Bewegungspläne („GANZ woandershin“, Z. 7). Daraufhin wenden sie sich von Tommy ab und nähern sich dem Exponat, nach dem Natalie ursprünglich gefragt hat (s.u. „Tiger“, Teil 2, \#4).

Mit anderen Worten: Tommys Weg durch den Raum, der ihn nicht zu einem nächsten Exponat führt, wird als abweichend qualifiziert und von den beiden Erwachsenen nicht gegangen. Die beiden Erwachsenen geben hier die Orientierung an einem räumlichen Zusammenbleiben als Gruppe auf, die für die Bewegungen im Rahmen des gemeinsamen Museumsbesuchs charakteristisch ist (s.o.), und gehen stattdessen zu dem Exponat, das als nächstes Etappenziel bestimmt worden ist. Das zeigt meines Erachtens, wie wichtig ihnen die Gestaltung des Wegs durch den Ausstellungsraum als ,Rundgang، ist, d.h. als Folge von Annäherungen an das jeweils nächste Exponat. Und es macht deutlich, dass sie versuchen, Tommy diese Bewegungsweise nahezubringen (oder in ihren Worten: ,vorzuschreiben').

In Teil 2 des Ausschnitts „Tiger“ tritt der Rundgang als ,verborgene Agenda“ noch deutlicher zutage:

\section{V0300, „Tiger“ Teil 2}

09 NA: \#eigentlich wenn man WEGschaut, $\# 4$
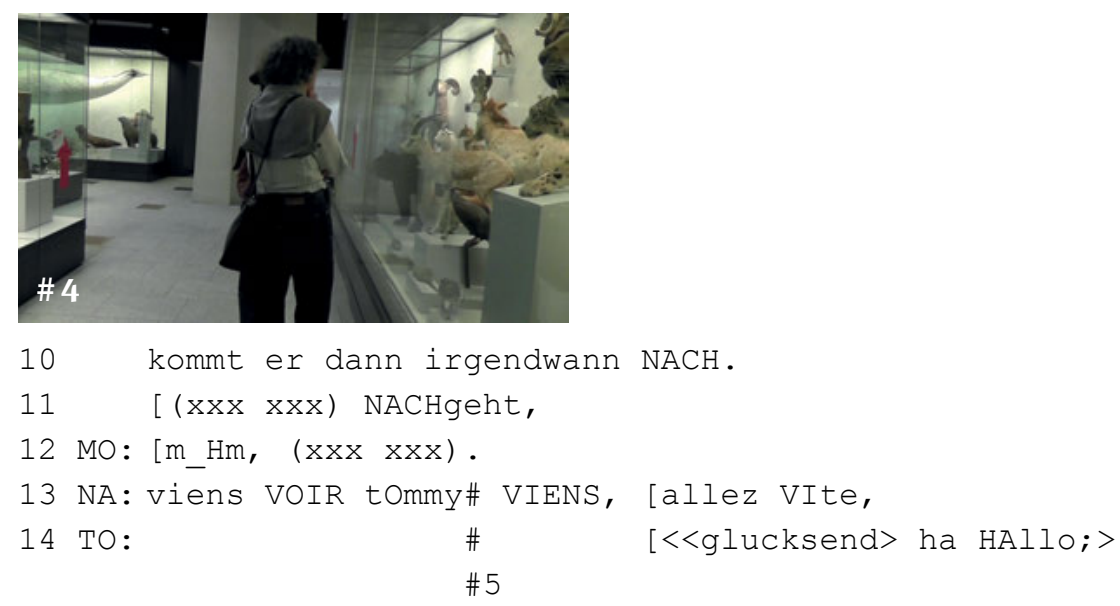


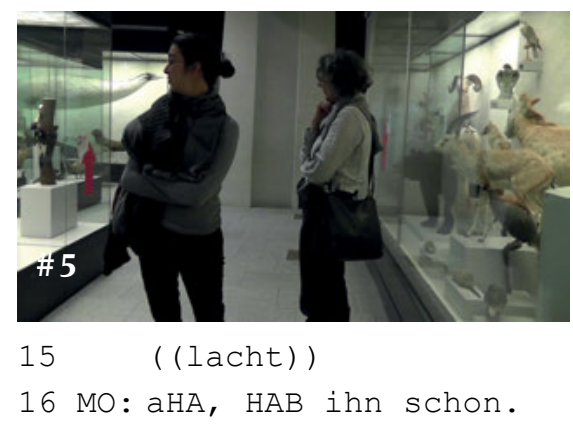

Hier offenbart Natalie den Plan, der hinter ihrer Hinwendung zum Exponat steht. Es handelt sich um eine Maßnahme, die Tommy zum Exponat locken soll, und damit zurück auf den ,rechten Pfad“ des Rundgangs: „eigentlich wenn man WEGschaut, kommt er dann irgendwann NACH.“ Das Ziel ist es, Tommy zu einer Position zu bewegen, von der aus er das Exponat betrachten kann („viens VOIR“, Z. 13).

Tommy hat die Entfernung zu seinen Begleiterinnen für ein Fangen- und Versteckspiel genutzt. Im dritten und letzten Teil des Ausschnitts „Tiger“ sehen wir, wie ihn seine Großmutter und Mutter zurück auf den Rundgang führen und somit sein abweichendes Raumverhalten korrigieren.

\section{V0300, „Tiger“ Teil 3}

17 NA: \#BIEN_on va aller voir le: tIgre et le crocoDILe; \#6

18 (--)

19 Viens VOIR, (--)

20 le \#tigre et le? Montre moi \#7
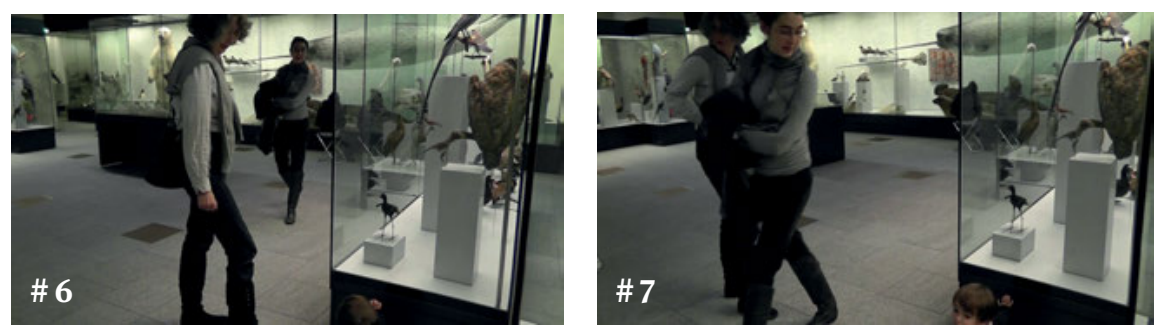

21 le tIG[re.

22 MO: [und was ist DA\#[S?

$23 \mathrm{NA}$ :

\# [momEnt \# 8 

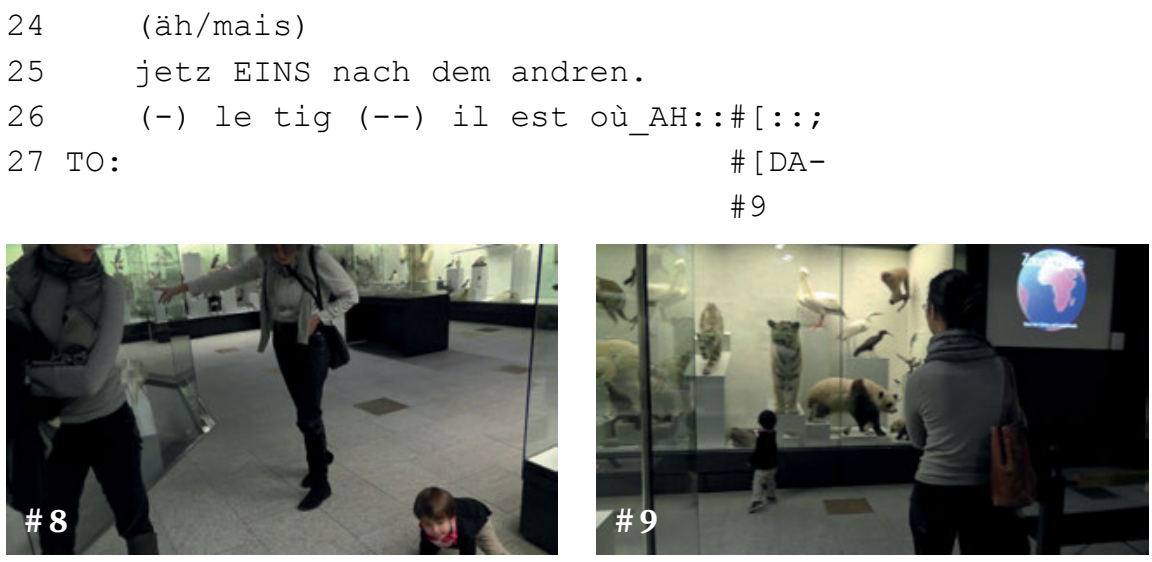

Standbild \#6 zeigt das Ende von Tommys Versteckspiel. Großmutter Mona hat ihn ,gefangen' und steht neben ihm, Mutter Natalie nähert sich den beiden. Sobald sie Tommy erreicht hat, erneuert sie ihren Versuch, das Kind zurück auf die von ihr definierte Etappe des gemeinsamen Rundgangs zu steuern. Durch die 1. Person Plural (,on va allEr“, Z. 17) und den Auftrag, ihr etwas zu zeigen („MONtre moi le TIGre“, Z. 20f.) entwirft Natalie die Beschäftigung mit dem Tigerexponat als eine gemeinsame Aktivität. Noch während die Mutter das Wort an ihren Sohn richtet, geht sie an ihm vorbei, wobei sie den Kopf so dreht, dass sie den Blickkontakt mit ihm aufrecht erhalten kann (\#7). So gelingt es ihr, das Kind gleichsam , hinter sich herzuziehen'.

Monas anschließender Beitrag bringt noch einen weiteren Aspekt des Rundgangs zum Vorschein: seine strikte sequenzielle Ordnung. Während Tommy sich mit seiner Mutter schon auf das Tigerexponat zubewegt, schlägt Mona ein alternatives Bewegungsziel vor: Sie zeigt auf ein Meerestierexponat und fordert Tommy auf, es zu identifizieren („und was ist DAS?“, Z. 22 und \#8). Doch dabei wird sie von Natalie unterbrochen, die in einem Account die Regel explizit macht, die dem Rundgang zugrunde liegt: „EINS nach dem andren.“ (Z. 25).

Nun wird Tommys ,Abweg' wieder in den unterbrochenen Rundgang integriert. Natalie signalisiert mit ihrem emphatischen „AH::::;“ (Z. 26), dass sie das gesuchte Exponat gefunden hat, und auch Tommy verdeutlicht, dass er das Gesuchte identifiziert hat, indem er so nah wie möglich an das Exponat herantritt und „DA-“ ruft (Z. 27 und \#9).

In den drei Teilen des Ausschnitts „Tiger“, die soeben analysiert worden sind, wird sichtbar, dass die Besucherinnen die Bewegungen durch den Ausstellungsraum als Abfolge von Bewegungen von Exponat zu Exponat organisieren. Der Weg des Kindes durch den Raum, der nicht auf das Erreichen des nächsten Exponats ausgerichtet ist und somit der Bewegungslogik des Rundgangs widerspricht, 
wird durch die beiden Frauen als ,Abweg' behandelt und wieder in den Rundgang überführt: Tommys ,Abweg‘ endet an genau dem Punkt, an dem Tommy den Rundgang verlassen hat, dem Tigerexponat. So kann die von den Frauen hervorgebrachte systematische Abfolge von Betrachtungspositionen nahtlos fortgesetzt werden. In „Tiger“ wird auch die gleichzeitige Beschäftigung mit mehreren Exponaten als Abweichung von der sequenziellen Logik des Rundgangs gekennzeichnet (in Natalies Account, s.o.). Eine solche Orientierung am systematischen Voranschreiten im Raum ist in meinem Korpus relativ häufig zu beobachten. ${ }^{13}$ Doch sind die Bewegungen einer Besuchergruppe auch dann noch als Teil eines Museumsbesuchs erkennbar, wenn die Gruppe sich nur einzelne Exponate aus dem Angebot ,herauspickt' und das Angebot im Raum nicht systematisch ausschöpft. Deshalb scheint mir ein systematisches, streng serialisiertes Vorwärtsgehen durch den Raum für die Musterhaftigkeit der Besucherbewegungen nicht konstitutiv zu sein.

Durch den Rundgang als Organisationsprinzip der Besucherbewegungen wird eine spezifische Deixis des Weiter und Zurück möglich gemacht, die für die beobachtete soziale Praxis grundlegend ist. Dies illustriert beispielhaft der folgende Ausschnitt (,Weiter“). Wir sehen eine Gruppe von Kindern aus einem Hort („Anna“, AN; „Chrigu“, CH; „Didier“, DI; und „Emil“, EM), die gerade zusammen mit ihrer Betreuerin „Barbara“ (BA) ein Computerquiz zu Lebensräumen und Ernährungsweisen von Tieren bearbeitet haben (s.a. Kesselheim 2012).

\section{V3210, „Weiter“}

01 BA: \#GÖMmer wIter. (--)

\#1
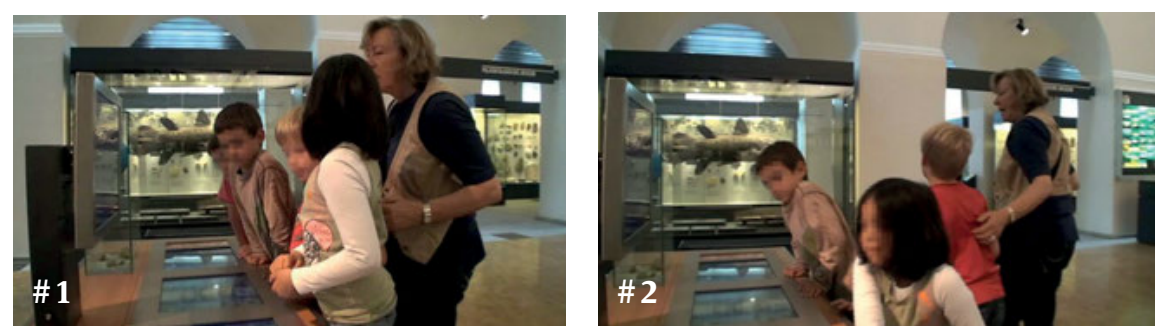

02 AN: $\mathrm{AH}:: ;=$

13 Auch Véron/Levasseur (1991) beobachten diese Form des Rundgangs in ihrem Material. Sie nennen dieses Bewegungsmuster mit einer Metapher aus dem Tierreich die Bewegungsart der ,Ameise‘. 

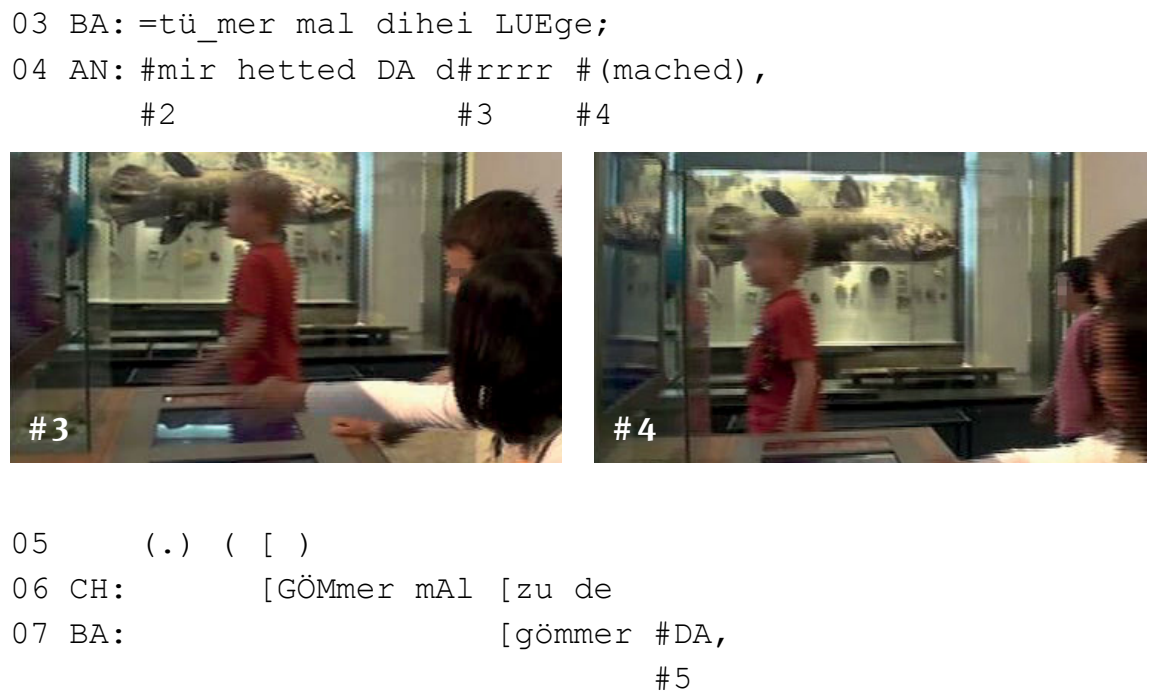

08 (.) chom DA; \#

\section{\# 6}
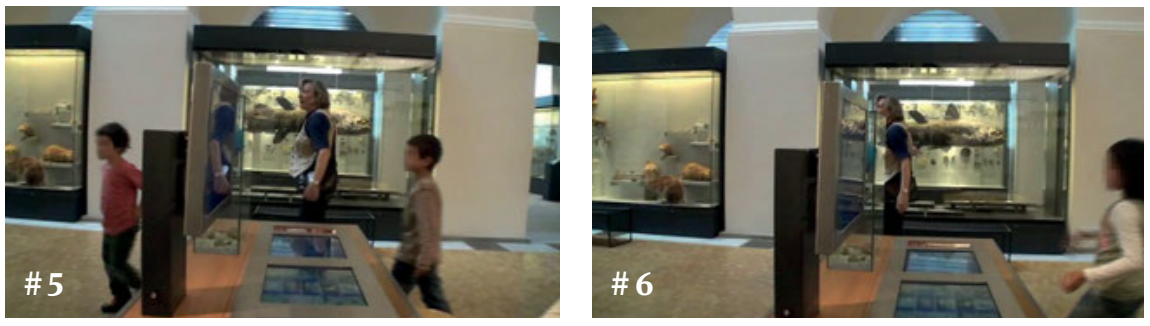

Mit ihrer Äußerung „GÖMmer wIter.“ (Z. 1) initiiert BA die Abwendung von dem Computerquiz. Sie tritt nach hinten aus der Formation heraus, die die Gruppe für das Quiz eingenommen hat (\#1). Sie dreht sich um und ,schiebt‘ eines der Kinder von der PC-Station weg, indem sie ihm ihre Hand auf den Rücken legt und so ihre Bewegung auf das Kind überträgt (\#2). Die Bewegungsfreiheit der Kinder ist durch den offenen Raum nicht eingeschränkt. Wir können deshalb die Bewegungen der Kinder also als ihre Interpretation des deiktischen Weiter verstehen. Wohin gehen die Kinder hier? Alle gehen (von der Kameraposition aus gesehen) nach links: in einen Raumbereich, den sie auf ihrem Rundgang noch nicht betreten haben. Allerdings meint ,wIter“ mehr als das. ,Weitergehen“ heißt: angemessene Betrachtungsziele finden, mit denen der bisherige Rundgang fortgesetzt werden kann. Sichtbar wird das etwa dann, wenn eine Neuausrichtung der Bewegung durch den Raum auf eine Veränderung der Blickrichtung folgt (wie im Fall von Chrigu, der zunächst einen Kopf leicht nach links dreht und gleich 
im Anschluss seine Laufrichtung ändert, \#3 zu \#4). Oder daran, dass die Kinder den Blick auf relativ weit entfernte Raumbereiche oder Dinge gerichtet haben (\#6) und diesen schnell und geradlinig zustreben. Auch erschließt sich die Ausrichtung des „wIter“ auf Betrachtungsziele im Raum aus den jeweiligen Endpunkten der Bewegungen: alle Bewegungen kommen schließlich vor einer Vitrine zu einheimischen Nagern zum Stillstand, wobei die Markierung des Bewegungsziels mit den oben beschriebenen Mitteln geschieht, also durch Herausstellung des Aufregenden oder Besonderen in Verbindung mit Verben des Sehens (vgl. Chrigus emotionalen Ausruf „IUeg mal wie dä A:knabberd_isch) bzw. durch eine Bezugnahme auf das Zielobjekt in Begriffen der in der Ausstellung präsentierten Wissenschaft („das isch en BibEr,“). Abb. 5-9 dokumentiert diesen Endpunkt der Bewegung durch den Raum.

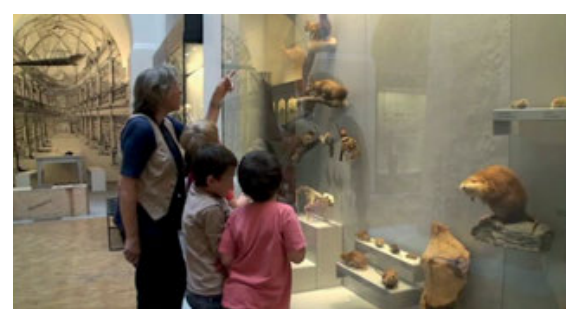

Abb. 5-9

In diesem Abschnitt haben wir gesehen, dass die Besucher ihre Bewegung durch den Ausstellungsraum als Rundgang, als Abfolge von Bewegungen hin zu noch nicht betrachteten Exponaten organisieren. Diese Abfolge ist derart musterhaft für den Museumsbesuch, dass auf ihr eine ganz eigene Deixis, die des Weiter (und des Zurück) aufsetzt, über die sich die Besucher bei ihren Bewegungen im Ausstellungsraum orientieren können. Die Verweisziele dieses Typs von Deixis lassen sich nicht mit Blick auf den Raum erschließen (wie im Fall von ,im Keller oder ,zum Faultier`) ebenso wenig wie mit Blick auf die Position des Sprechers (wie bei ,rechts', ,da hinten'). Das Verweisziel ergibt sich aus den bisherigen Stationen des gemeinsam konstruierten Rundgangs durch den Ausstellungsraum.

Mit „Abfolge“ ist allerdings nicht gemeint, dass die Besucher jedes einzelne Ausstellungsstück in der von der Ausstellung suggerierten Reihenfolge betrachten müssen (wenn es eine solche Reihenfolge gibt). Entscheidend ist, dass unter all den prinzipiellen Möglichkeiten, den Raum zu konstruieren, eine solche gewählt wird, die durch das sukzessive Ansteuern immer eines weiteren Betrachtungsziels organisiert wird. In 5.4 werden wir sehen, dass der Rundgang die Herstellung eines übergeordeten thematischen Zusammenhangs unterstützen kann: indem er es den Besuchern erlaubt, ein Exponat als neuen Fall eines schon beobachteten Phänomens einzuordnen (z.B. „das isch AU wieder so_nen mammutzahn.“, 
Patrick in V8816). Allein durch die Suggestion eines Zusammenhangs, den die körperliche Erfahrung einer kontinuierlichen Bewegung mit sich bringt (s. beispielsweise Heath/vom Lehn 2001: 286f. oder vom Lehn/Heath/Hindmarsh 2001: 198).

\subsubsection{Formen der Bewegung im Museum}

Nicht nur die auf die Exponate hin organisierte Bewegung durch den Ausstellungsraum ist für den Museumsbesuch charakteristisch. Zur Beschreibung der Bewegungen, die zur Praxis des gemeinsamen Museumsbesuch gehören und die die Teilnahme an dieser Praxis für Beobachter wiedererkennbar machen, gehört auch eine Beschreibung ihrer Geschwindigkeit: das oben erwähnte gemessene Schreiten. Dass dieser Aspekt der musealen Bewegungen für die Besucher von Bedeutung ist, zeigt sich daran, dass er in Besuchen mit Kinderbeteiligung immer wieder Gegenstand von Korrekturen oder gar expliziten Thematisierungen wird. Ein besonders aufschlussreiches Beispiel für Letzteres findet sich in dem folgenden, „Ayla“ benannten Ausschnitt. Es handelt sich um einen Ausschnitt aus einem Gespräch, das ich mit einer Primarlehrerin („Estelle“, ES) geführt habe. Die Lehrerin hat ihre Schüler in Dreiergruppen eingeteilt, die durch das Paläontologische Museum gehen. Dabei müssen sich je drei Kinder an den Händen halten, was deren Bewegungsfreiheit merklich herabsetzt (s. Abb. 5-10).

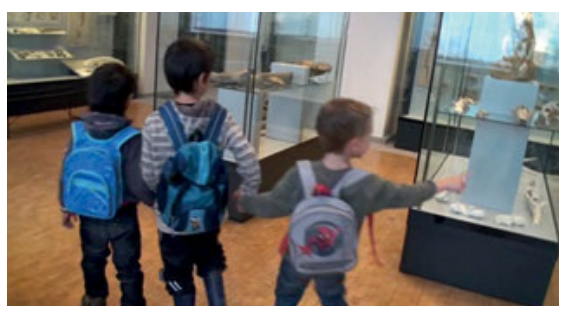

Abb. 5-10

Vor dem Einsetzen des transkribierten Ausschnitts bin ich einer solchen Dreiergruppe mit der Kamera gefolgt und so nach einiger Zeit wieder bei der Lehrerin angelangt. Während ich weiter die Kinder filme, erläutert mir die Lehrerin, der Museumsbesuch ermögliche es ihren Schülern, „kulTUR kennen zu lernen“. Ich betone daraufhin, dass das Paläontologische Museum dafür „SEHR geeignet“ sei, weil die Kinder dort große Freiheiten besäßen. Hier setzt das Transkript ein:

\section{V3400, „Ayla“ Teil 1}

01 ES: es Is aber auch äh:m SCHWIErig für: für die kInder- 


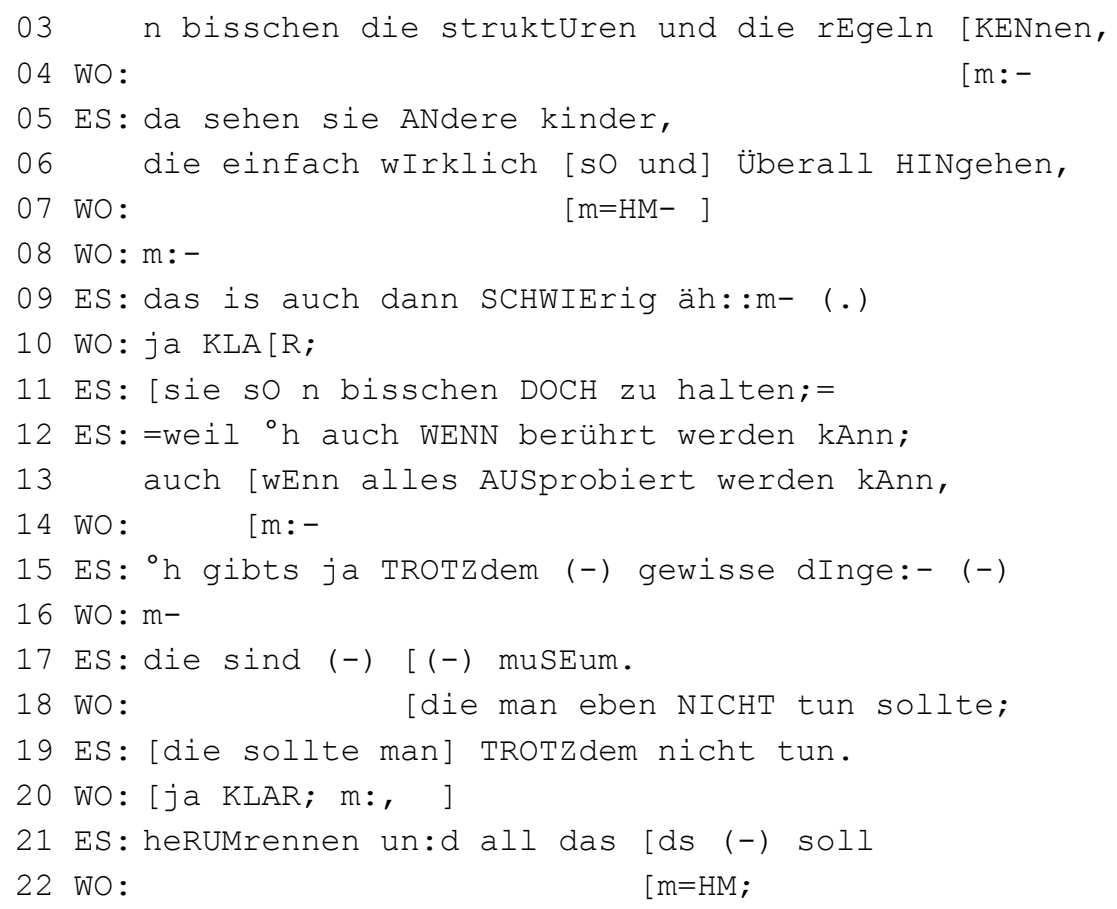

Von welcher Schwierigkeit Estelle spricht, wird erst im Verlauf ihres Beitrags deutlich.

- Anfangs ist lediglich klar, dass es sich bei der angesprochenen Schwierigkeit um etwas handelt, das dem Tenor meiner Äußerung widerspricht (,aber“, Z. 1) bzw. einen von mir vernachlässigten Gesichtspunkt darstellt (,auch“, Z.1). Dann führt Estelle den Aspekt der Regelkenntnis ein, durch die Kontrastintonation in „ICH“ als Gegensatz markiert: „ICH hab wirklich gern wenn sie $\mathrm{n}$ bisschen die struktUren und die rEgeln KENnen, “ (Z. 2f.).

- Der Hinweis auf die ,anderen Kinder‘, die „einfach wIrklich sO und Überall HINgehen, “ (Z. 6) macht deutlich, um welche Regeln es Estelle hier geht: um Regeln des Sich-im-Museumsraum-Bewegens, die gerade das „einfach [...] sO“ Gehen und das „Überall HINgehen“ verhindern. In der Folge reformuliert Estelle die Schwierigkeit in „sie sO n bisschen DOCH zu halten;“ ( Z. 11). Was also schwierig ist, ist die Einhaltung der Regeln angesichts des Verhaltens der ,anderen Kinder', deren Bewegungen nicht regelkonform sind.

- Aus ihrer folgenden Äußerung lässt sich Weiteres zum Inhalt der von ihr angeführten Regeln erfahren. Als ,Dinge', die möglich sind (vgl. die Betonung auf „kAnn“, Z. 12), aber nicht getan werden dürfen, nennt Estelle exemplarisch das „heRUMrennen“ (Z. 21), also die Art der Fortbewegung durch den 
Ausstellungsraum. Sich angemessen zu verhalten heißt also konkret, nicht durch den Ausstellungsraum zu rennen.

- Besonders interessant ist aber Estelles Formulierung „dInge:- (-) die sind (-) muSEum.“ (Z. 15-17). Denn durch diese Formulierung macht Estelle deutlich, dass das Einhalten der Berührungs- und Bewegungsregeln für sie mehr ist als nur ein beliebiger Aspekt des Verhaltens im Museum. Die Regeln des Raumverhaltens machen die Institution Museum ganz eigentlich aus, so dass Estelle sie mit der Institution Museum gleichsetzen kann.

Genau hier unterbricht sich die Lehrerin und ruft ein Kind zur Ordnung, das gegen diese Bewegungsregeln verstoßen hat:

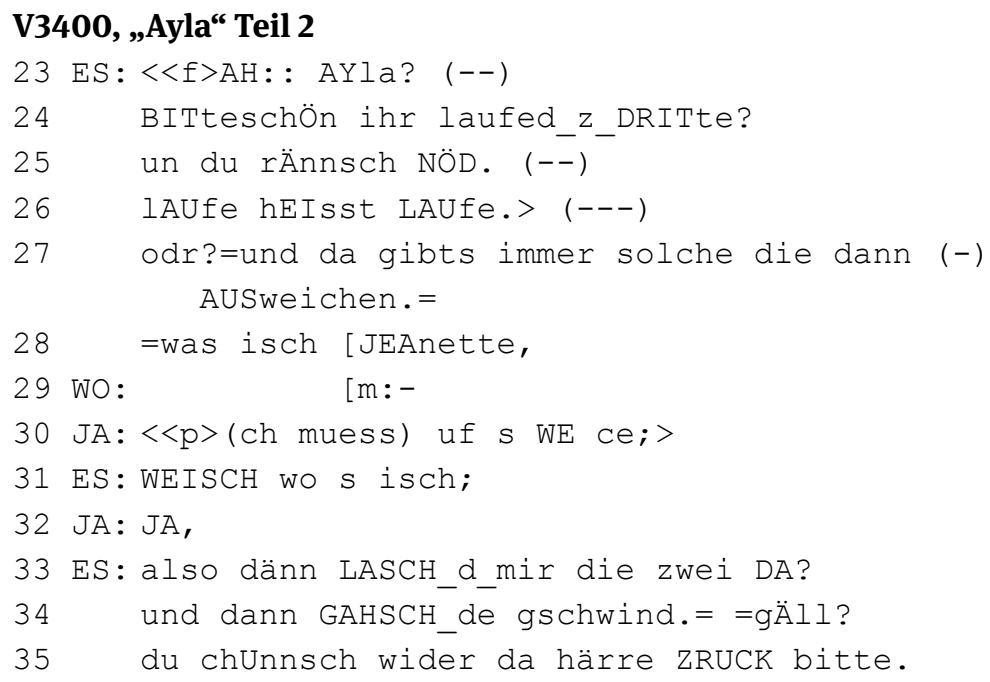

In diesem zweiten Teil des Ausschnitts „Ayla“ formuliert Estelle explizit die Regeln, denen die Bewegungen der Kinder durch den Raum entsprechen sollen. Das ,Rennen“ wird als unangemessen verboten („du rÄnnsch NÖD.“, Z. 25) und das Verhalten der Ermahnten als ein Umgehen dieser Regeln charakterisiert („AUSweichen“, Z. 27). Dem ,Rennen“ stellt Estelle in ihrem Beitrag die langsamere Bewegung des ,Laufens“ gegenüber. Durch das Format „x heißt x“ kennzeichnet sie ihre Ermahnung, sich langsam durch den Raum zu bewegen, als Erinnerung an eine bereits besprochene Regel, an die sie jetzt erinnert. Wir haben es hier also mit einem Account zu tun, in dem eine Interaktionsbeteiligte die Regeln ihres Raumverhaltens zum Thema macht.

Genau in diese Erklärung der musealen Bewegungsregeln hinein bittet eines der Kinder darum, auf die Toilette gehen zu dürfen. Während Estelle bisher ener- 
gisch einfordert hat, die Bewegungen beim Museumsbesuch sollten langsam erfolgen („du RÄnnsch NÖD“, Z. 27) - eine Bewegungsart, die sie durch die ,Kopplung“ von je drei Kindern zu einer Gruppe unterstützt (,ihr laufed_z_DRITte“, Z. 24, Abb. 5-10), soll der Gang auf die Toilette, der das Kind „Jeanette“ (JA) durch den Ausstellungsraum des Zoologischen Museums führt, „gschwind“ (Z. 34) erfolgen. Für den Gang auf die Toilette, die das Kommunikationsangebot im Ausstellungsraum nicht nutzt, ist eine schnelle Bewegung, für den Rundgang, der die Ausstellung im Sinn des gemeinsamen Museumsbesuchs nutzt, ein langsames „LAUfe“ angemessen.

Bei der Beantwortung der Frage, ob eine beobachtete Bewegung durch den Ausstellungsraum Teil eines Rundgangs durch das Museum ist oder nicht, spielt aber nicht nur die Geschwindigkeit des Gehens eine Rolle. Wichtiger als diese ist der sicht- und hörbare Bezug auf die Exponate. Das möchte ich anhand von zwei ,problematischen' Fällen knapp illustrieren. Der erste Fall soll zeigen, dass schnelle Bewegungen durch den Raum nicht notwendigerweise bedeuten, dass die Betreffenden den Raum nicht im Sinne der Ausstellungskommunikation nutzen. Der zweite Fall dagegen belegt, dass ein langsames Gehen durch den Raum nicht unbedingt bedeuten muss, dass der Ausstellungsraum im Sinne der Ausstellungskommunikation genutzt wird.

Zum ersten Fall. Ein Großvater („Victor“, VI), eine Großmutter („Anna“, AN) und ihre zwei Enkel im Primarschulalter („Melanie“, ME, und „Luca“, LU) sind dabei, ein Quizblatt mit dem Titel „Wer bin ich - Einheimische Tiere (Erdgeschoss)“ auszufüllen (V3027). Dieses Quizblatt besteht aus einer Reihe von nummerierten Detailfotos von Tierpräparaten, die im Ausstellungsraum gefunden werden sollen und deren Namen es in ein Kreuzwortgitter einzutragen gilt. Gemeinsam haben die vier Gruppenmitglieder den Luchs als nächstes zu suchendes Objekt bestimmt und schwärmen nun im Ausstellungsraum aus, wie Abb. 5-11 zeigt.

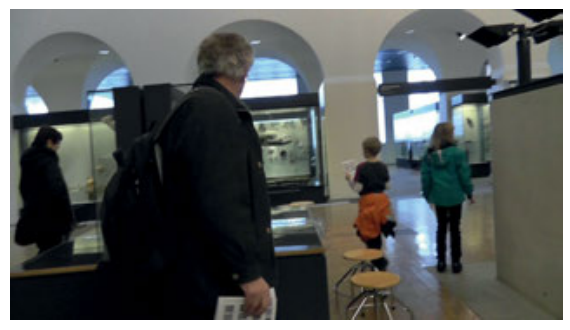

Abb. 5-11

Dabei laufen sie wesentlich schneller durch den Ausstellungsraum als die übrigen Besucher. Auch entfernen sie sich deutlich weiter voneinander, als dies sonst zu beobachten ist, bis dahin, dass Luca in einen Gang des Paläontologischen 
Museums hineingeht, während die anderen in dem zum Zoologischen Museum gehörenden Gebäudeteil bleiben, und sich dabei für längere Zeit aus dem Sichtfeld der anderen Gruppenmitglieder entfernt.

Trotz alledem sind ihre Bewegungen als Teil eines gemeinsamen Museumsbesuchs zu erkennen. Dafür sorgen die Gruppenmitglieder, indem sie ihr Gehen mit ihren Körpern deutlich als ein Suchen gestalten - etwa indem sie das Quizblatt hochhalten und den Blick über dessen oberen Rand in den Raum hinaus richten (s. Luca auf Abb. 5-11 o.) - vor allem aber, indem sie auffallend häufig auf ihr gemeinsames Suchobjekt sprachlich Bezug nehmen.

- So wiederholt die Großmutter ,en LUCHS-“ genau in dem Moment, in dem sie und ihre Enkel die zunächst eingeschlagene Suchrichtung aufgeben und eine andere Richtung einschlagen.

- Beim nächsten Halt, im Eingangsbereich des paläontologischen Ausstellungsteils, erinnert Victor seine Frau und seine Enkeltochter (Luca ist vorgerannt) an die weiterhin bestehende Suchaufgabe: „en LUCHS müend_mer sueche.“, und der ,Rückzug، aus diesem Ausstellungsbereich wird mit Verweis auf den Luchs kommentiert und mit dessen Fehlen rechtfertigt (Melanie: „dA häts AU chei luchs.“).

- Mit der Suche nach dem Luchs wird auch Lucas Abwesenheit rechtfertigt: Victor ruft ihm nach „HÄSCH_en?“. So macht er dessen Abwesenheit zu einem Teil der gemeinsam definierten (Museums)Aktivität, und gerade nicht zu einem ,Abweg“ wie im oben analysierten Ausschnitt „Tiger“. Lucas Entfernung von der Gruppe bedeutet keinesfalls, dass er nicht mehr am gemeinsamen Museumsbesuch teilnimmt. Im Gegenteil, sie ist ein Zeichen für seine Suchanstrengungen.

Wir konnten hier also sehen, dass die Bewegungen einer Gruppe durch den Raum auch dann noch als Realisierung eines gemeinsamen Museumsbesuchs gesehen werden können, wenn sie nicht den oft beschriebenen Formen der langsamen Fortbewegung durch den Raum entsprechen. Entscheidend ist offenbar die köperlich dargestellte oder verbalisierte Ausrichtung auf ein legitimes Bewegungsziel: ein Exponat. Das Fortbestehen dieser Ausrichtung auf museale Bewegungsziele signalisieren sich die Mitglieder der Gruppe insbesondere dann, wenn diese Ausrichtung für ihre Bewegung durch den Museumsraum unmittelbare Konsequenzen hat (Richtungsänderungen, ein Stehenbleiben oder Weitergehen). Solange auf anderem Wege verdeutlicht wird, dass die gemeinsame Orientierung an der kommunikativen Nutzung des Ausstellungsraums bestehen bleibt (z.B. dass also beispielsweise die Suche nach dem Luchs fortgesetzt wird), kann sich selbst ein einzelnes Gruppenmitglied zeitweise aus dem Gesichtsfeld der Gruppe entfernen, 
ohne dass dies als Abbruch des gemeinsamen Museumsbesuchs interpretiert würde.

Bei dem zweiten angekündigten Fall (aus Video V1024), handelt es sich um eine Gruppe von Studenten, deren Status als Museumsbesucher problematisch ist, obwohl sie sich im Schlendertempo durch den Ausstellungsraum bewegen (vgl. Treinens berühmte Charakterisierung des Museumsgangs als ,kulturelles Schaufensterbummeln', Treinen 1981: 25). Die Gruppe hat im Raum nach dem Abguss eines Mammutbabys gesucht und befindet sich jetzt auf dem Weg zum Ausgang, wobei sie sich gegenseitig Erlebnisse mit Tieren erzählen. Die folgende Reihe von Standbildern dokumentiert ihren Weg zum Ausgang:

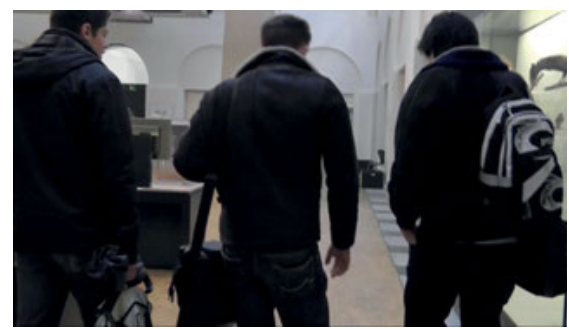

Abb. 5-12

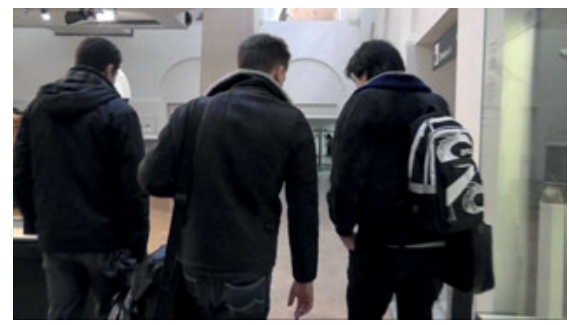

Abb. 5-14

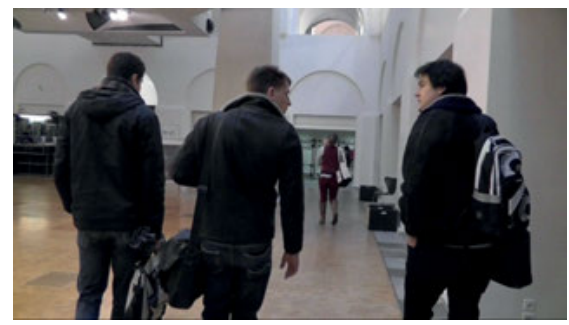

Abb. 5-16

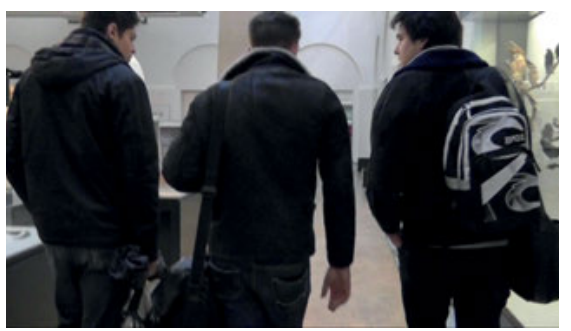

Abb. 5-13

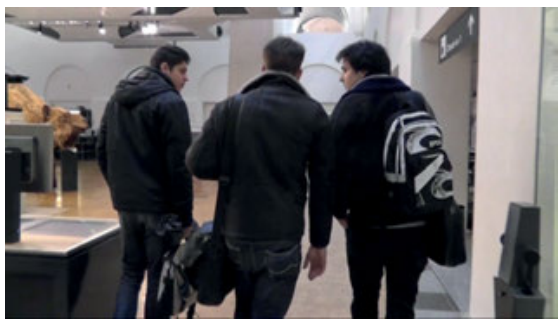

Abb. 5-15

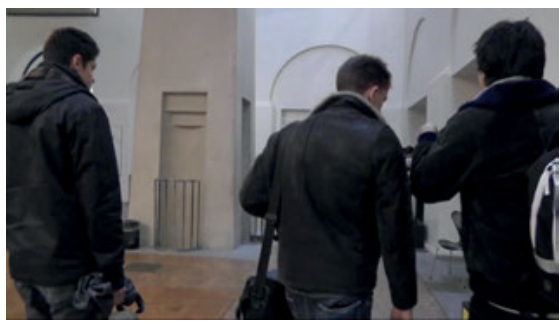

Abb. 5-17 


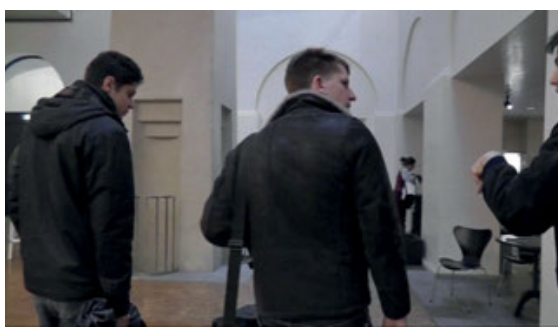

Abb. 5-18

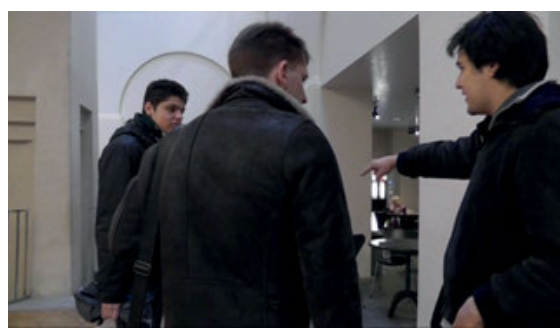

Abb. 5-20

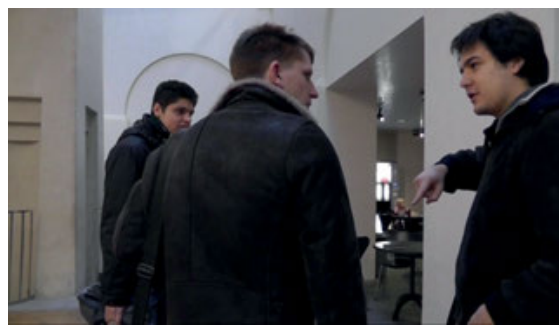

Abb. 5-22

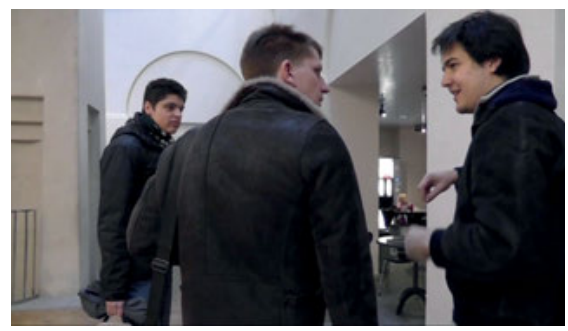

Abb. 5-19

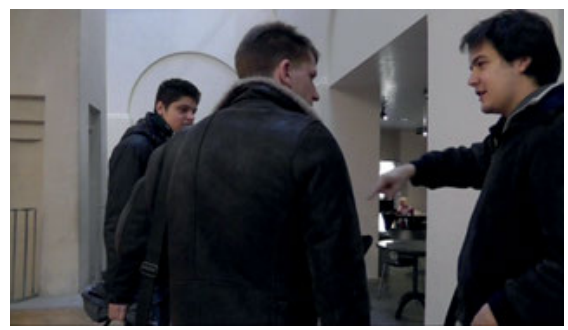

Abb. 5-21

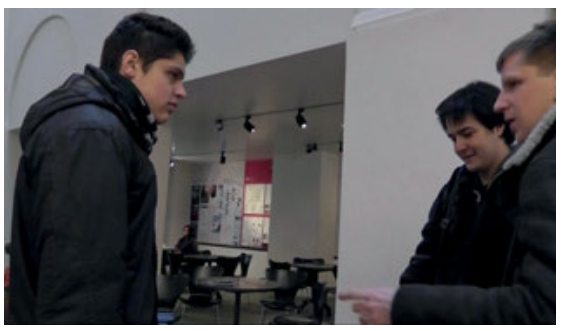

Abb. 5-23

Was an diesen Bildern (bzw. der Videoaufnahme) ist für den Eindruck verantwortlich, dass sich die Gruppe zwar physisch im Museum befindet, dass sie den Ausstellungsraum aber nicht im Sinne der Ausstellungskommunikation nutzt?

Zwar nutzt die Gruppe einen Bereich des Ausstellungsraums zum Gehen, der durch seine langgezogene Form als ,Vektor` und damit als für das Gehen geeignete Zone markiert ist (s.o. 3.3). Doch gibt es eine Reihe von Unterschieden zu dem Raumverhalten, das in der großen Mehrheit der von mir aufgenommenen gemeinsamen Museumsbesuche zu beobachten ist.

- Während sich die Blicke der Besucher bei ihrem Rundgang im Regelfall an den Angeboten des Raums orientieren, ist auf den Abbildungen 5-12-5-16 
zu erkennen, dass die Blicke der drei Studierenden entweder auf die jeweils anderen gerichtet sind oder, nach der Kopfhaltung zu urteilen, kurz vor den Füßen der Gehenden auf den Boden treffen (s.u. 5.3). Auch sind im Unterschied zu der üblichen Bewegungsweise Änderungen der Kopf- und Blickrichtung zeitlich nicht mit Änderungen der Laufrichtung verbunden (s. als Kontrast den schon untersuchten Ausschnitt „Weiter“ in 5.2.4).

- Die Bewegung durch den Raum endet nicht in einer Verweilzone vor einem Exponat (s.o. 5.2.3), wie es durch das multimodale Kommunikationsangebot im Ausstellungraum nahegelegt wird (s.o. 4.3.2), und die Gruppenmitglieder bilden auch nicht die häufig dort anzutreffende I-Formation, die ihnen die gemeinsame Betrachtung eines Exponats ermöglicht. Stattdessen können wir auf den Standbildern Abb. 5-17 bis 5-19 eine geschlossene, hufeisenförmige Formation beobachten, die die Aufmerksamkeit der Gruppe im Innern der Formation konzentriert. Auch die Geste des Studenten ganz rechts im Bild ist nicht auf den Raum außerhalb der Formation gerichtet, sondern beschreibt wie die Abbildungen 5-20 bis 5-22) belegen - einen Kreis ins Zentrum des von ihren Körpern umstandenen „o-space“ (Ciolek/Kendon 1980: 243).

Ausgehend von diesen abweichenden Beispielen lässt sich nun die bisherige Beschreibung der für den gemeinsamen Museumsbesuch charakteristischen Bewegungen durch den Ausstellungsraum erweitern. Die Bewegungen der Besucher durch den Ausstellungsraum müssen nicht nur in gemessenem Tempo erfolgen. Sie müssen auch erkennbar auf die Objekte oder Texte im Ausstellungsraum bezogen sein. Stehen diese beiden Aspekte im Widerstreit, scheint - wie weitere, ähnlich gelagerte Fälle bestätigen - die Ausrichtung der Bewegungen auf die Exponate für die Herstellung des museumsspezifischen Bewegungsraums wichtiger zu sein als der Aspekt der angemessenen Geschwindigkeit.

\subsubsection{Fazit: der museale Bewegungsraum}

Wir haben gesehen, dass die Besucher ihre Bewegungen durch den Ausstellungsraum so aufeinander abstimmen, dass sie als gemeinsame Bewegungen durch den Raum sichtbar werden. Dabei stellen sie eine räumliche Nähe zu ihren MitBesuchern her, die sie sowohl in den mobilen als auch in den stationären Phasen des Museumsbesuchs aufrechterhalten.

Die Bewegungen, die zur sozialen Praxis des gemeinsamen Museumsbesuchs gehören, zeichnen sich aber nicht nur durch ihre Ko-Orientierung aus: Charakteristisch für sie ist in besonderem Maße, dass die Besucher ihre Bewegungen mit Bezug auf die Exponate organisieren. In den Videoaufnahmen meines Korpus 
begegnen uns die Exponate sowohl als Motiv als auch als Ziel von Bewegungen durch den Raum. Wie sich in den Analysen zeigte, ist allerdings nicht das Exponat selbst der Endpunkt der Bewegungen, sondern ein Bereich vor diesem. Diesen Bereich habe ich in 3.3.2 als „Verweilzone“ bezeichnet, weil sich in ihm die statischen Phasen des Museumsbesuchs abspielen. Die genaue Position und die innere Strukturierung Verweilzonen ergibt sich aus der Funktion des Exponats innerhalb beiden anderen musealen Teilräume, des Betrachtungs- und des Handlungsraums, die in den nächsten Abschnitten untersucht werden.

Die einzelnen Bewegungen im Ausstellungsraum erwiesen sich als eingebettet in eine übergeordnete Struktur: eine zyklische Abfolge von mobilen Phasen (hin zum nächsten Exponat) und stationären Phasen (vor dem Exponat). In meinen Analysen habe ich exemplarisch gezeigt, wie die Besucher diesen ,Rundgang' interaktiv hervorbringen und wie sie ihn gegen ,Abwege‘ verteidigen.

Ein Aspekt der Bewegungen durch das Museum, der in der Literatur wie auch in den Einschätzungen der Akteure des Felds eine große Rolle spielt, stellte sich in meiner Untersuchung dagegen als sekundär heraus: die Geschwindigkeit des Gehens durch den Ausstellungsraum. Hier konnte die Analyse Hinweise darauf erbringen, dass der sicht- oder hörbare Bezug auf das kommunikative Angebot im Ausstellungsraum für die Einschätzung der Bewegungen wichtiger ist als eine langsame Gangart.

\subsection{Die Herstellung des musealen Betrachtungsraums}

\subsubsection{Ausgangspunkt}

Im Museum ist vieles, wenn nicht alles, darauf ausgerichtet, dass bestimmte im Raum arrangierte Dinge (Exponate, erläuternde Texte) von Besuchern gesehen und betrachtet werden. Aus Produzentenperspektive bezeichnet der Museologe Friedrich Waidacher die Museumsausstellung deshalb als einen Fall von ,präsentativer Kommunikation“: eine Kommunikation, die davon lebt, dass dem Besucher Dinge ,vor Augen gestellt‘ werden, die dieser im Lauf des Museumsbesuchs betrachtet (Waidacher 2005). Das Sehen, und speziell das Betrachten von Exponaten im (Kunst-)Museum ist aus zahlreichen Perspektiven untersucht worden. Untersuchungen und Theorien gibt es aus der Kunstgeschichte, Kulturwissenschaft, Psychologie, Sozialwissenschaft und vielen Disziplinen mehr (z. B. Bryson 2001, Bal 2008, Gombrich 1984, Emmison/Smith 2000). Unmittelbar einschlägig für das hier verfolgte Untersuchungsinteresse sind auch die Arbeiten zum Museum von Christian Heath, Dirk vom Lehn und Kollegen (vom Lehn/Heath 2007a, Hindmarsh/Heath 2003, Heath/vom Lehn 2004, Heath et al. 2002) sowie von Heiko 
Hausendorf (Hausendorf 2010) zum gemeinsamen Betrachten von Exponaten in Museen und Galerien. Charles Goodwin hat sich schon in den 90er Jahren dem profesionellen Sehen gewidmet und dabei untersucht, wie Merkmale der materiellen Umwelt in fachliches Handeln eingebettet werden. Ein weiterer Anknüpfungspunkt ist die konversationsanalytische Forschung zur Herstellung von ,joint attention“, die die multimodale Herstellung geteilter visueller Aufmerksamkeit als Hervorbringung der Interaktionsbeteiligten untersucht (s. Kidwell/Zimmerman 2007, Mondada 2014). Dabei kommen zum Teil auch Eye-Tracking-Brillen zum Einsatz, um das Zusammenspiel von Blickbewegungen, deiktischen Gesten und Sprache untersuchen zu können (Stukenbrock 2018; im Museum: Stukenbrock 2020; Balantani/Lázaro 2021).

Weniger Untersuchungen gibt es zum Zusammenhang von Hören und Raum (s. Coyne/Parker 2009 oder Turner/McGregor/Turner 2009) ${ }^{14}$ oder zur Relevanz anderer Sinneskanäle zur Herstellung unterschiedlicher Wahrnehmungsräume (s. hier etwa Hall 1976 zu Räumen, die durch die Wahrnehmung der Körperwärme oder des Atems des Gegenübers hergestellt werden). Breidenstein (2004) weist auf die Notwendigkeit hin, bei der Analyse zu berücksichtigen, dass es grundsätzlich mehrere Wahrnehmungsräume gleichzeitig geben kann, die - abhängig von den physikalischen und physiologischen Bedingungen der Wahrnehmung - unterschiedlichen Strukturprinzipien gehorchen und auf verschiedene Weise miteinander überlagert sein können.

Der folgende Abschnitt untersucht nun, wie die Interaktionsbeteiligten im Verlauf ihres Museumsbesuchs museumsspezifische „Wahrnehmungsräume“ hervorbringen (Hausendorf 2010). Dabei frage ich: Welcher Zuschnitt des Wahrnehmungsraums ist für den Ausstellungsraum musterhaft. Und welche Art des Sehens ist für die Aktivität des gemeinsamen Museumsbesuchs typisch und angemessen, so dass sie dazu beiträgt, die räumliche Umwelt um die Interaktionsbeteiligten herum als Ausstellungsraum zu konstruieren und deren gemeinsames Handeln als Nutzung eines Ausstellungsraums im Rahmen eines gemeinsamen Museumsbesuchs. Wir werden sehen, wie Besucher Schritt für Schritt - und unter Beteiligung verbaler wie nicht-verbaler Ressourcen - einen gemeinsamen Wahrnehmungsraum mit einem Exponat als visuellem Aufmerksamkeitszentrum herstellen, „den musealen Betrachtungsraum“ (s.a. Winiger, im Druck). Ich werde zeigen, dass dieser Betrachtungsraum nicht nur durch die Richtung der Blicke definiert wird, sondern ebenso durch das besondere ,Timing‘ der Blicke sowie durch eine museumsspezifische Art des Sehens.

14 Ober-Heilig (2015: 117) stellt einen Zusammenhang her zwischen hohen Museumsräumen mit einer halligen Akustik und dem ,implizite[n] Ruhegebot“ in Museumsausstellungen. 


\subsubsection{Die Herstellung des gemeinsamen Wahrnehmungsraums}

Im nun folgenden Analyseabschnitt werde ich untersuchen, wie die Besucher während ihres Gangs durch die Museumsausstellung einen gemeinsamen Wahrnehmungsraum herstellen und welche Aspekte dieser gemeinsamen Herstellung und des gemeinsam hergestellten Raums musterhaft für die soziale Praxis des gemeinsamen Museumsbesuchs sind.

Die Analysen dieses Abschnitts beginnen mit einer Aufnahme, die schon in 5.2 untersucht worden ist. Sie zeigt Vater Patrick und Tochter Nina auf dem Weg zum frei stehenden Mammutexponat im Erdgeschoss des Zoologischen Museums (der Ausschnitt „Mammut“ fasst die schon oben untersuchten Ausschnitte „Riesengroßes Tier“ und „Herumturnen“ zusammen, allerdings teilweise mit anderen Standbildern).

\section{V8816, „Mammut“}

01 NI: <von Ferne> oh PApi-> (-) pApi chUmm ma go LUEge; =

02 =ich han_s rIIsesgrosses [TIER\# gsehn; (--)

03 PA: [JA, \#

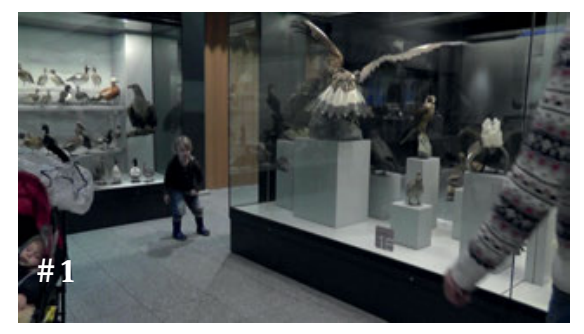

04 NI: da CHUMM ma-

05 ich han $\mathrm{n}$ MAMmut gsehn.

06 n MAMmut. (-)\# (-)

\#2

07 n MAM\# [mut.

08 PA: \#[en MAMmut;

\#3 

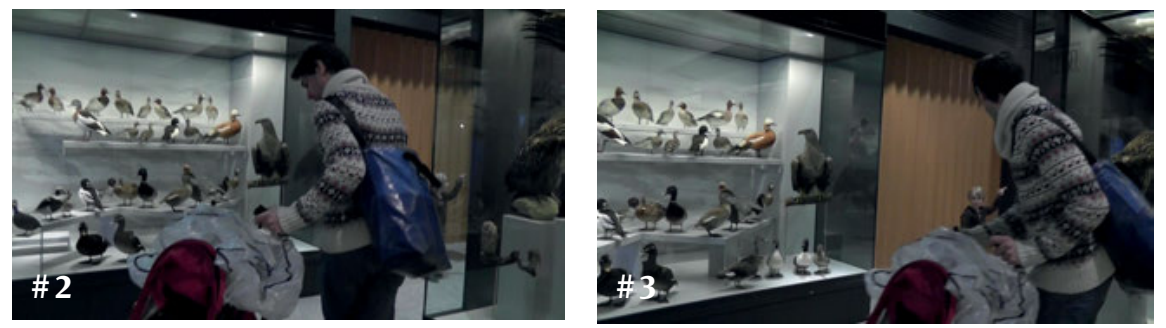

$09 \quad(2.0)$

10 NI: ((klatscht in die Hände) \#((klatscht, 3 sek.)) \# 4

$11 \mathrm{PA}: \# \uparrow{ }^{\top} \mathrm{OH}::$ ja. (4.9)\#

\# 5

$12 \mathrm{NI}:<<\mathrm{p}>$ (chammer) $>$ sich \#grAd dra HEbe.

\# 7
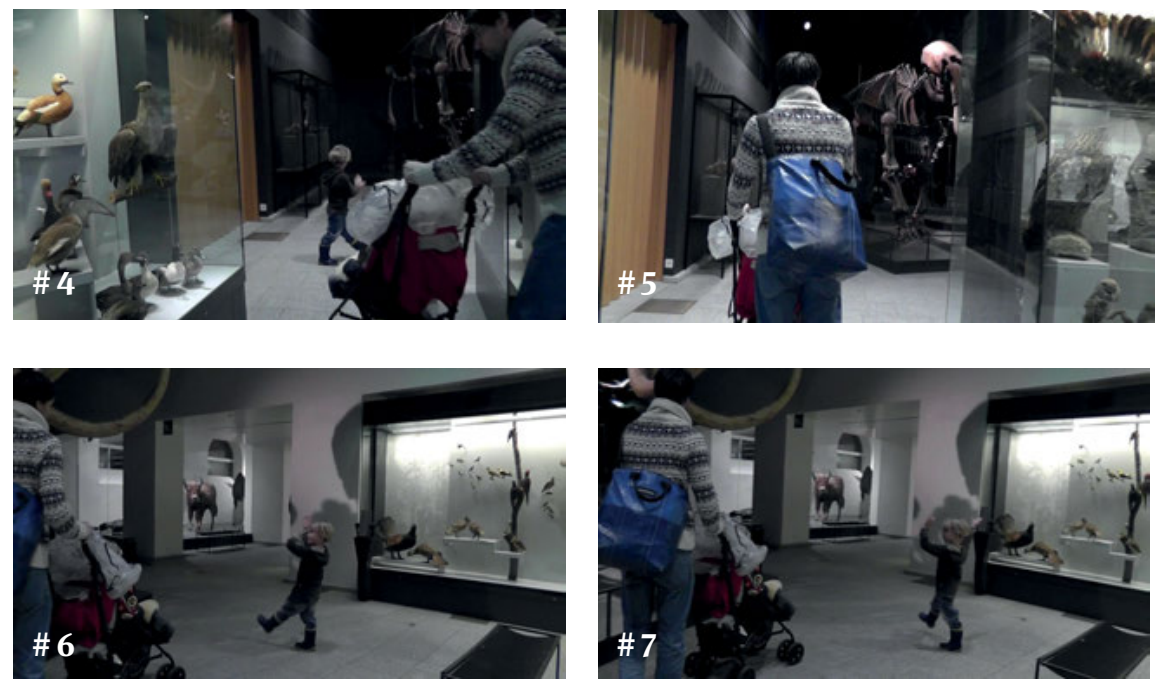

$13(4.9)$

14 PA: ja das dÖrf mä NÖD,

15 abr (-) die sind eso gross dass mer CHÖNNT;

16 GÄLL?

$17 \mathrm{NI}:\langle\langle\mathrm{p}\rangle \mathrm{JA} ;>$

18 PA: chönnt mä richtig druf UMeturne.

$19(---)$

20 NI: wemm (.) wemmer WÜRkli fescht druf Umetrampled_dänn 


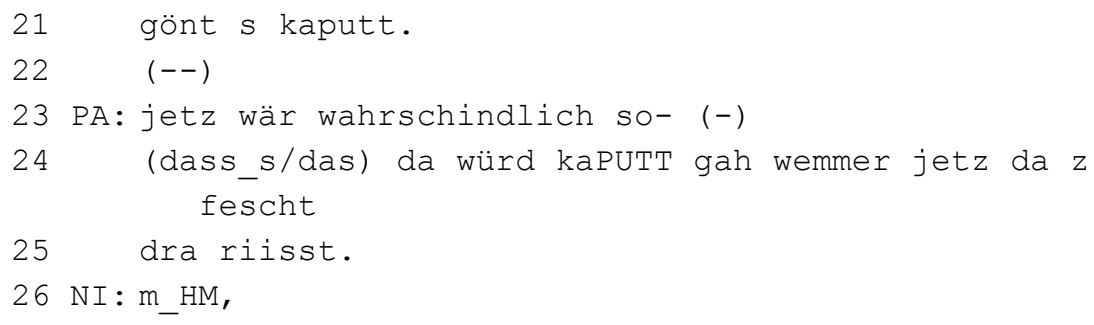

Im vorigen Analysabschnitt haben wir bereits festgestellt, dass dem Sehen im Rahmen des gemeinsamen Museumsbesuchs eine besondere Stellung zukommt: Indem sie ihren Begleitern signalisieren, dass es an einem Exponat etwas (Interessantes, Aufregendes, Wundersames) zu sehen gibt, können Museumsbesucher ihre Begleiter dazu animieren, sich durch den Ausstellungsraum zu dem fraglichen Exponat zu bewegen.

Die Koordination der Wahrnehmungen der Besucher beginnt daher nicht erst, wenn die Bewegung der Besucher in die stationäre Phase übergeht. Sie setzt ein mit der Definition des Bewegungsziels (das eben auch ein Betrachtungsziel ist). Ausschnitt „Mammut“ zeigt das gut:

- Der Aspekt des Sehens ist schon bei der Definition des Mammuts als Bewegungsziel thematisch: „,chUmm ma go LUEge;= =ich han_s rIIsesgrOsses TIER gsehn;“, Z. 1f.). Und die Bewegungsimplikation von Ninas Zeigegeste, die man in \#2 und \#3 sehen kann, ergibt sich gerade aus der Tatsache, dass Patrick von seinem Standpunkt aus das von Nina gezeigte Objekt nicht sehen kann. Ninas Geste projiziert die Richtung, in der der noch herzustellende gemeinsame Wahrnehmungsraum liegen soll. Dieser Effekt ergibt sich speziell aus der Tatsache, dass die Ausrichtung des Rumpfs von der Ausrichtung von Kopf und Arm abweicht. Mit Kopf und Arm leitet sie Patricks Blick quasi von sich ab und lenkt ihn auf das angezeigte Ziel, während sie mit ihrem restlichen Körper zwischen dem Zeigeziel und der Position ihres Vaters ,vermittelt".

- Nina geht nun auf das Mammut zu (\#4) beziehungsweise, wie man später sieht, auf den Bereich vor dem Mammut, der durch die freie Fläche am Boden als Verweilzone markiert ist.

- Wenige Sekunden später hat Patrick eine Position erreicht, von der aus er das Mammut sehen kann (\#5), was er mit „'OH:: ja.“ (Z. 11) zu erkennen gibt (s.o.). Nun könnte er an dieser Stelle stehenbleiben, denn er sieht ja das von Nina angekündigte Mammut. Doch wenn Patrick das Mammut von dieser Position aus betrachtete, wären sein und Ninas Betrachten des Mammuts nicht als gemeinsames Betrachten erkennbar. Auch wäre ein gegenseitiges Wahrnehmen des Betrachtens unmöglich: Nur Patrick könnte gleichzeitig das Mammut betrachten und sehen, dass und wie Nina dass Mammut betrachtet. 
Umgekehrt wäre das nicht möglich: Nina müsste sich entscheiden, ob sie das Mammut, oder aber Patricks Betrachtung des Mammuts sehen möchte.

- Offensichtlich reicht es für die Zwecke der beiden Besucher nicht aus, dass beide alleine das Mammut sehen: Denn Patrick geht weiter auf Ninas Position zu, bis er kurz vor Nina zum Stehen kommt. Dabei zeigt sich eine Symmetrie in den Kopfbewegungen von Patrick und Nina. Während Patrick sich annähert, blickt er zu seiner Tochter, und sie wendet ihm den Kopf halb zu (\#6). Mit seinem Stehenbleiben wendet Nina den Kopf nach vorne, zum Mammut, und nahezu zeitgleich schaut auch ihr Vater zu dem Exponat (\#7).

- Beide Besucher haben ihre Blicke auf das Mammut ausgerichtet - die Verweilzone ist damit zu einer Betrachtungszone geworden - und sie stehen dabei so nahe beieinander, dass ihr Betrachten des Mammuts als gemeinsame Aktivität gesehen werden kann. Anders als in dem Moment der Annäherung, der in \#5 dokumentiert ist, können nun beide den jeweils anderen über die periphere Sicht wahrnehmen und ihn so beim Betrachten des Exponats kontrollieren.

- In diesem Moment haben sie einen gemeinsamen Wahrnehmungsraum erzeugt. Das ist nicht nur an ihrer ko-orientierten körperlichen Ausrichtung auf das Exponat zu erkennen. Erkennbar ist es auch daran, dass beide unproblematisch mit deiktischen Ausdrücken auf Elemente in diesem Wahrnehmungsraum verweisen können (Ninas „dra HEbe“ - ,daran festhalten“, Z. 12 - und Patricks „die sind eso gross“, Z. 15). ${ }^{15}$

Hieran kann man eine Reihe von allgemeinen Beobachtungen zur Herstellung des gemeinsamen Wahrnehmungsraums durch Museumsbesucher machen.

Die Herstellung des gemeinsamen Wahrnehmungsraums wird hier interaktiv ausgehandelt. Die Initiative für seine Herstellung geht von Nina aus, aber Patrick richtet seine Wahrnehmung (ebenso wie seine Bewegung durch den Raum, die er mit Hinblick auf die Wahrnehmungsmöglichkeiten gestaltet) auf das von Nina identifizierte Ziel aus. Diese Ausrichtung wird nicht nur einmal vorgenommen, sondern dynamisch an die sich verändernden Positionen, Körperhaltungen und Zeigegesten von Nina angepasst, aber auch an die jeweiligen Möglichkeiten und Beschränkungen für die Wahrnehmung des anderen, die jeweils von konkreten Formationen im Raum ausgehen (s.o. zu \#5).

15 Kaum ist der gemeinsame Wahrnehmungsraum etabliert, nutzen ihn die beiden Besucher für ihre Beschäftigung mit dem Exponat (hier: für die Erarbeitung von Regeln des korrekten Umgangs mit dem Mammutskelett). Die Herstellung des musealen Betrachtungsraums mündet also in die Etablierung und Nutzung des Raums im Sinne eines musealen Handlungsraums. Dieser Aspekt wird in 5.4 ausgearbeitet werden. 
Wenn es auch um die Hervorbringung eines visuellen Wahrnehmungsraums geht, nutzen die Besucher hier nicht nur sichtbare Ausdrucksressourcen (Positionierung im Raum, Ausrichtung des Körpers und speziell des Kopfs, Blicke, deiktische Gesten), sondern in gleichem Maße auch Sprache. Bezieht man die sprachlichen Äußerungen der Besucher nicht in die Analyse ein, kann man den gemeinsamen Weg durch den Ausstellungsraum nicht als Weg hin zur gemeinsamen Betrachtung eines Exponats verstehen. Das ist nur möglich, wenn man Ninas sprachliche Definition des Mammutexponats als Betrachtungsziel und Patricks Akzeptieren dieses Ziels (,en MAMmut.“, Z. 5) mitberücksichtigt.

Das Sehen spielt nicht erst dann eine Rolle, wenn die Bewegung der Besucher in die stationäre Phase übergeht. Vielmehr ist es von Beginn der mobilen Phase an relevant. Patrick macht nämlich seine Bewegungen als Reaktion auf Ninas Projektion einer gemeinsamen Betrachtungsmöglichkeit kenntlich. Und das Sehen spielt auch am Ende der gemeinsamen Koorientierungsanstrengungen eine Rolle. Die Möglichkeit des gemeinsamen Betrachtens (und die gegenseitige Kontrolle dabei) definiert die Positionierung der Interaktionspartner im Raum. Durch diese Ausrichtung auf die gemeinsame Betrachtungsmöglichkeit erhält die durch die Bewegung durch den Ausstellungsraum definierte „Verweilzone“ (s.o. 5.2) zugleich den Charakter einer „Betrachtungszone“.

\subsubsection{Die Exponate als Zentrum des musealen Betrachtungsraums}

Für den geteilten Wahrnehmungsraum, der im Verlauf des gemeinsamen Museumsbesuchs hervorgebracht wird, ist nun spezifisch, dass er nicht primär auf die gegenseitige Wahrnehmung der Interaktionspartner ausgerichtet ist. Diese stehen in der Regel nebeneinander vor dem Exponat und nehmen sich nur am Rand ihres jeweiligen Gesichtsfelds wahr. Das dokumentiert das folgende, sehr einfach gehaltene Blicktranskript. ${ }^{16}$ Es zeigt die Blickausrichtung der beiden Interaktionsteilnehmer in je einer Spur (ni, pa) synchron zur jeweiligen Sprecherzeile (NI, PA). Mit einem Minuszeichen ist die Blickausrichtung auf das Mammut notiert, ein „p“ bzw. ein „n“ identifiziert die Momente, in denen Nina oder PA zu ihrem Gegenüber blicken.

16 Eingeklammerte Bereiche repräsentieren Momente, in denen der fragliche Sprecher nicht im Bild ist. - Die Videodaten erlauben keine genauen Blickanalysen (dazu wären EyeTracking-Daten nötig gewesen, wie sie Stukenbrock 2018, 2020 im Museum nutzt), sondern lediglich Annäherungen an die tatsächlichen Augenbewegungen. Deshalb wurde auch auf eine Transkription nach den von Rossano genutzten Konventionen (Rossano 2013) verzichtet. 


\section{V8816, „Herumturnen“ (Blicktranskript)}

$01 \mathrm{NI}:\langle<\mathrm{p}\rangle$ (chammer) sich $\rangle$ grAd dra HEbe.

ni: -------------------------ppppp--

$\mathrm{pa}:---------------------------------n n n$

02 PA: ja das dÖrf mä NÖD,

pa: --------------------

ni: --------------------

03 PA: abr (-) die sind eso gross dass mer CHÖNNT;

pa: --------------------nnnnnnnnnnnnnnnnnnnn---

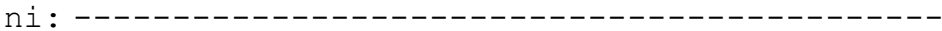

04 PA: GÄLL?

pa: ------

ni: ------

$05 \mathrm{NI}:\langle<\mathrm{p}>\mathrm{JA} ;>$

ni: ---------

pa: $--------n$

06 PA: chönnt mer richtig druf UMmeturne.

pa: nnnnnnnnnnnnnnnnnnnnnnnnnnnnnnnnnnn

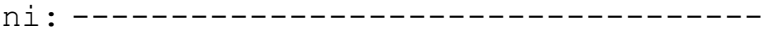

07 NI: ( (nickt)) ((nickt))

ni : pppppppppp-----------

pa: nnnnnnnnnnnnnnn------

08a NI: wemm (.) wemmer WÜRkli fescht druf Ummetrampled dänn

ni:

pa:

08b NI: gönt s kaputt.

ni : ppppppppppppp

pa: nnnnnnnnnnnnnnn

09 PA: ( (nickt)) ( (nickt))

pa: nnn----------------

ni: pppppppppppp------

10 PA: jetz wär wahrschindlich so- (-)

pa: -------------------------------

ni:----------------------------------

11aPA: (dass_s/das) da würd kaPUTT gah wenn d jetz da z fescht

pa:

ni : 


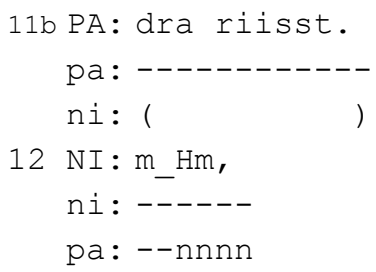

Dieses Blicktranskript macht unmittelbar augenfällig, wie stark die Blicke der beiden Interaktionspartner auf das Mammut aufgerichtet sind.

So ruht der Blick des aktuellen Hörers weit häufiger auf dem Mammut als auf dem Sprecher, was nach den Beschreibungen der frühen Blickforschung zu erwarten gewesen wäre (s. dazu Rossano 2013: 312). Und wenn der aktuelle Sprecher zum Hörer blickt, trifft sein Blick, im Gegensatz zu der von Goodwin (1980: 275 und 287) beschriebenen Norm des Blickverhaltens in Interaktion, kaum je auf den Blick des Hörers (Z. 7, 8f.). Stattdessen sieht er, wie jener das Exponat betrachtet. Das ist besonders dort auffällig, wo ein Sich-gegenseitig-Sehen für die Kommunikation eigentlich funktional schiene. So schaut Nina, die pantomimisch das Am-Exponat-Schaukeln vorführt, erst ganz am Ende ihrer ersten Äußerung zu Patrick (Z. 1), obwohl Patricks Blick eine notwendige Voraussetzung für die kommunikative Wirksamkeit ihrer Pantomime gewesen wäre. - Spekulieren lässt sich höchstens, dass Patrick Ninas ,Pantomime‘ über seine periphere Sicht wahrnehmen kann.

Besonders auffällig ist hier, dass die Abwendung des Blicks vom Gegenüber an keiner Stelle als Hinweis auf ein Sich-Zurückziehen aus der gemeinsamen Aktivität behandelt wird (als Indiz für ein „disengagement“, Goodwin 1980) oder als Initiative zur Beendung einer Sequenz (,making a bid for closure“, Rossano 2013: Abschnitt 3.2). In Z. 3 beispielsweise wendet Patrick als der aktuelle Sprecher seinen Blick von Nina ab und lässt ihn auf dem Mammut ruhen (Z. 4). Gleichzeitig signalisiert er jedoch verbal, dass er eine Reaktion von Nina erwartet („GÄLL?“, Z. 4), und signalisiert so, dass er gerade nicht auf die Beendigung der Sequenz abzielt (s.a. seine Fortsetzung des Themas in Z. 6. Offensichtlich, so möchte ich argumentieren, wird im Rahmen des gemeinsamen Museumsbesuchs die Abwendung des Blicks von einem Interaktionspartner nicht als „disengagement“ oder „bid for closure“ behandelt, wenn der Blick nicht $\mathrm{zu}$ irgendeinem beliebigen Punkt im Raum wandert, sondern auf ein Exponat gerichtet wird.

Die besondere Relevanz des Exponats zeigt sich in diesem Transkriptausschnitt auch in Momenten, in denen ein Akteur nach einem Blickkontakt ${ }^{17}$ seinen

17 In Z. 1 kommt möglicherweise kein Blickkontakt zustande, allerdings sieht Patrick höchst- 
Blick vom anderen abzieht und ihn zum Mammutexponat lenkt. Tatsächlich lässt sich beobachten, dass die Blickzuwendung zum Exponat den Blick des anderen unmittelbar danach ebenfalls in Richtung Exponat ,zieht‘. Dies geschieht beispielsweise in Z. 1: Patrick schaut zu seiner Tochter, als diese schon wieder ihren Kopf zum Mammut dreht, und Sekundenbruchteile danach wendet auch er seinen Blick dem Ausstellungsstück zu. Ähnliches geschieht in Z. 7: Patrick hat den Faden der subversiven Nutzungsmöglichkeiten weitergesponnen (Z. 6), und Nina nickt als Reaktion darauf (Z. 7). In diesem Moment treffen sich Patricks und Ninas Blicke. Aber noch während Nina ein zweites Mal nickt, dreht sie sich schon wieder dem Mammut zu, gefolgt von einer gleichläufigen Bewegung des Vaters (ebenfalls Z. 7). Offensichtlich ,zieht‘ Ninas Hinwendung zum Mammut Patricks Blick hinter sich her (zwei Fälle des von Stukenbrock 2020 beschriebenen „speaker gaze following“). Der nächste Blickkontakt zwischen den Gesprächspartnern erfolgt erst gegen Ende von Ninas Beitrag (Z. 8a und b), und wieder wartet einer der beiden Gesprächspartner das Ende der Reaktion nicht ab, sondern blickt zum Mammut, gefolgt von den Blicken des anderen (Z. 9): Schon während seines ersten Nickens wendet sich Patrick wieder dem Mammut zu, und sein zweites Nicken führt er vollständig in Richtung Mammut aus. Er signalisiert damit Nina, die seine Reaktion beobachtet, dass das Mammut weiterhin im Zentrum der Aufmerksamkeit steht. Die Blickorientierung in dieser Gesprächsphase ist also ganz wesentlich auf das Exponat ausgerichtet, während der Interaktionspartner nur punktuell in den Blick genommen wird. Insbesondere scheint sich mir die Relevanz des Exponats darin zu zeigen, dass die Blickabwendung vom Interaktionspartner nicht als Anzeigen eines „disengagement“ gewertet wird, wenn der Blick auf das Exponat gerichtet wird.

Im Blicktranskript lässt sich eine gewisse Asymmetrie erkennen: Patrick schaut häufiger zu Nina als diese zu ihm. Diese Asymmetrie hat ihr Gegenstück in der Positionierung der beiden Besucher im Raum, die auf Abb. 5-24 (auf der nächsten Seite) dokumentiert ist. Schon mit seiner Fuß- und Beckenstellung ist der Vater weniger eindeutig auf die Betrachtung des Mammuts ausgerichtet als die Tochter. Seine Körperfront ist nicht vollständig auf das Mammut, aber auch nicht vollständig auf die Tochter ausgerichtet. Vielmehr nimmt er eine Mittelposition zwischen Mammut und Tochter ein. Das erlaubt es ihm, an der gemeinsamen Betrachtung des Exponats teilzunehmen und gleichzeitig die Aktivitäten seiner Tochter im Blick zu behalten oder die Wirkung seiner Worte auf die Tochter zu kontrollieren, ohne dazu seinen Körper hin- und herdrehen zu müssen. Dies scheint mir ein räumlicher Ausdruck seiner Rolle als „visit manager“ (Kelly 2007: 289-292) zu sein: Er besucht nicht nur die Museumsausstellung, sondern über-

wahrscheinlich bei seiner Blickzuwendung auf Nina, dass diese ihn zuvor angeschaut hatte. 
prüft und organisiert auch den Umgang seines Kindes mit der Ausstellung (dazu mehr in 5.4 unten).

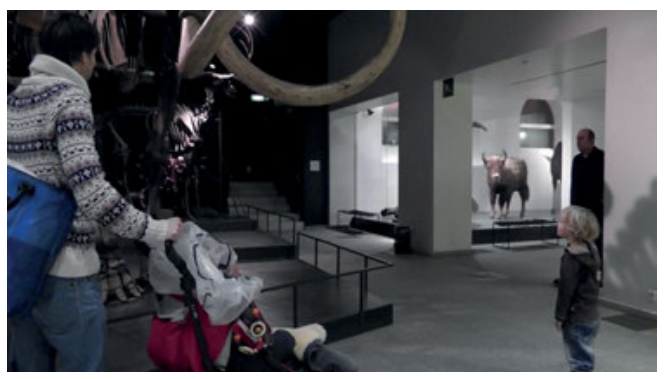

Abb. 5-24

Die Tatsache, dass sich im Zentrum des gemeinsamen Wahrnehmungsraums hier Exponate befinden, verleiht dem gemeinsamen Wahrnehmungsraum den besonderen Charakter, der ihn als spezifischen Wahrnehmungsraum für die Zwecke des gemeinsamen Museumsbesuchs ausweist: als „musealen Betrachtungsraum“.

Die zentrale Rolle, die die Exponate, begleitende Texte und Ausstellungshilfsmittel wie die Vitrine für den gemeinsamen Wahrnehmungsraum spielen, wird besonders deutlich, wenn man der Frage nachgeht, welche Aktivitäten als „first actions“ (vom Lehn 2013) zur Auflösung des musealen Betrachtungsraums führen können. Hier zeigt sich: Während es viele Dinge im Überschneidungsbereich von Patricks und Ninas Gesichtsfeldern gibt, von denen sich die Interaktionsbeteiligten ohne Folgen für das Interaktionsgeschehen abwenden können, stellt die Abwendung von einem Exponat eine Gefahr für das Fortbestehen des gemeinsamen Betrachtungsraums dar. Diese Tatsache belegen die folgenden drei Transkriptausschnitte „Läck! (kurz)“, „Fundort“ und „Enten“.

\section{V8816, „Läck!“(kurz)}

$$
\begin{array}{llllll}
\# 1 & (0.4) & \# 2 & (0.2) & \# 3 & (0.2)
\end{array}
$$
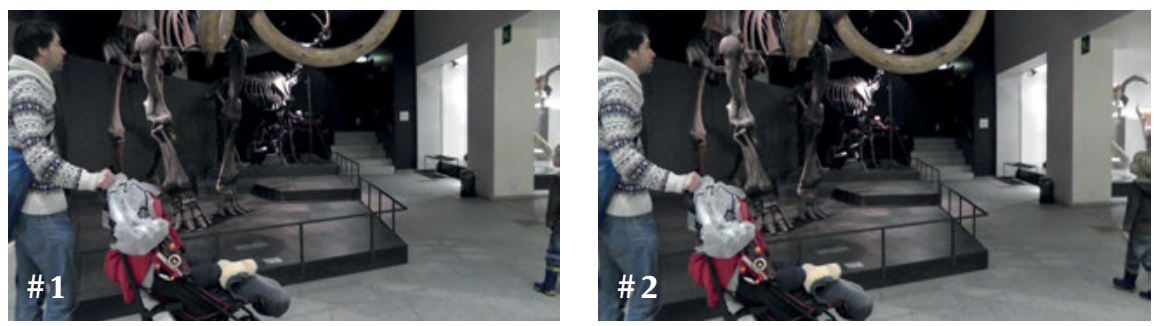


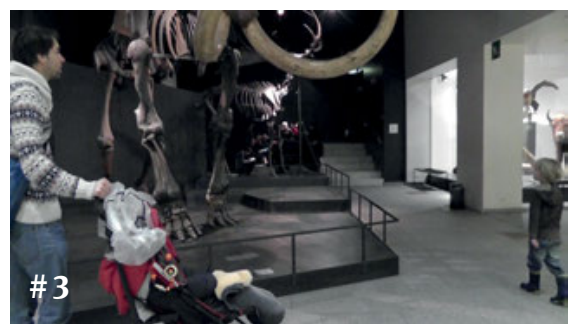

01 NI: (xxx) \#LÄCK\#4

$02 \quad(-) \#(-)$

\# 5
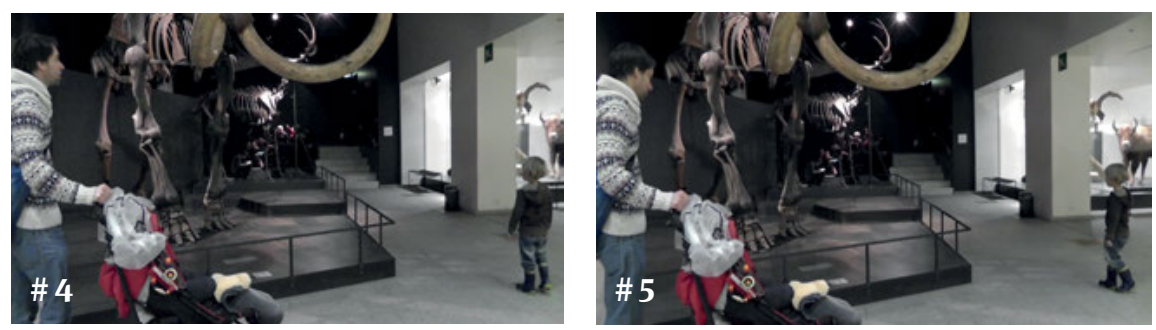

03 PA: \#oh LUEG emal \#was da- \#(-) \#(-)

\#6 \#

\#8 \#9
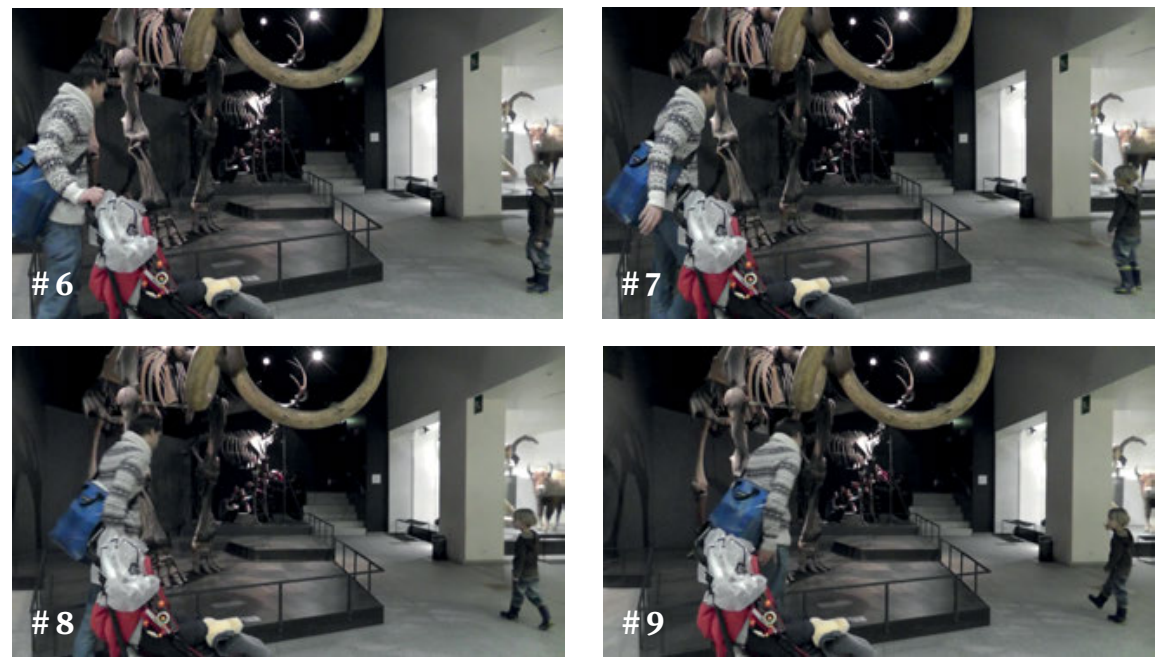

04 PA: DU. (-)

LUEG emal.\# 


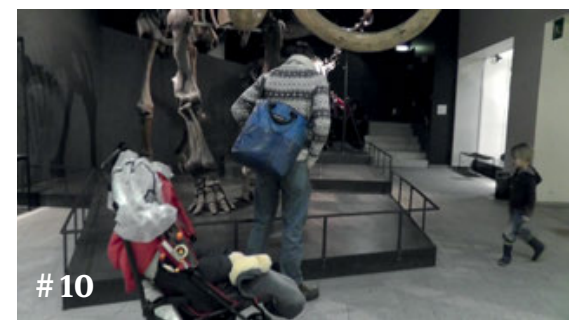

Im Ausschnitt „Läck!“ ist Ninas Beteiligung am gemeinsamen Betrachten so lange unproblematisch, bis sie ihren Kopf in eine Richtung dreht, von der aus sie das Mammut-Arrangement nicht mehr sehen kann.

- Obwohl sie sich schon vorher vom Vater wegbewegt (\#1-3), erscheinen ihre Schritte solange als auf das Mammut-Arrangement hin orientiert, bis ihr Blick deutlich an diesem Arrangement vorbeigeht (\#4-6) Dabei spielt keine Rolle, dass Blick und Bewegung durchaus auf etwas gerichtet sein könnten, das auch der Vater von seinem Standpunkt aus sehen kann. Aber dieses Etwas ist nicht mehr das Exponat oder ein erläuternder Text.

- Es ist sicher kein Zufall, dass Patricks Versuch, eine Auflösung der stationären Betrachtungsphase zu verhindern, genau dann beginnt, wenn Ninas Blick und Bewegung sich nicht mehr auf das Mammutexponat beziehen lassen. (Er weist auf etwas Sehenswertes direkt vor ihren Augen hin und beginnt, auf einen Punkt in seiner Nähe zuzugehen, wobei seine schiefe Kopfhaltung vermuten lässt, dass es sich um einen Text handelt, dessen Orientierung im Raum er mit dem Kopf übernimmt).

Ninas Zeigegeste, die auf etwas außerhalb des Mammut-Arrangements weist (\#7), scheint mir einen noch stärkeren Hinweischarakter zu besitzen als der Blick. Denn die Zeigegeste wirkt auch dann noch als klares Indiz für Ninas Abwendung vom gemeinsamen Betrachtungsraum, als sich Nina mit Blick und Körper schon wieder zu dem sie rufenden Vater wendet bzw. zu dem Objekttext, auf den jener sichtbar seine Aufmerksamkeit gerichtet hat (\#8, 9).

Auch im nächsten Transkriptausschnitt („Fundort“) ist es eine Abwendung vom Exponat (und dem gemeinsam genutzten Objekttext), die die Auflösung des gemeinsamen Betrachtungsraums nach sich zieht.

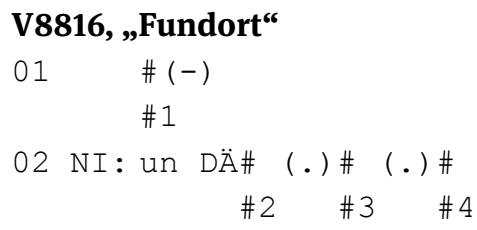



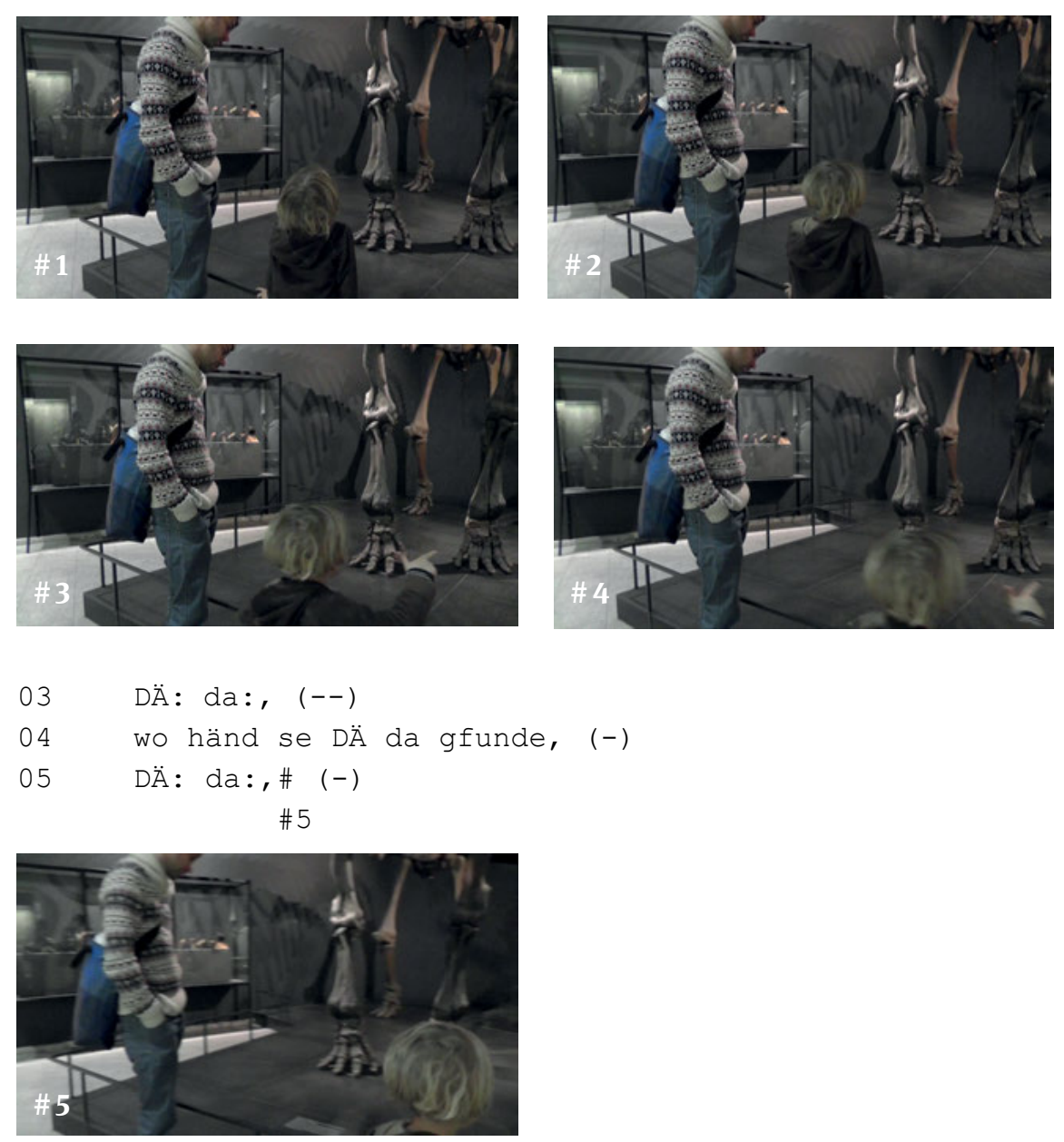

06 pAp (i) wo händs DÄ da gfunde,

Die Auflösung des gemeinsamen Betrachtungsraums beginnt im Ausschnitt „Fundort“ mit Bewegungen, die dazu führen, dass Nina Schild und Exponat nicht mehr im Sinne der Ausstellungskommunikation nutzen kann.

- Mit einer ersten schnellen und damit auffälligen Bewegung, dem Senken ihres Kopfs, unterbricht Nina die zuvor bestehende Blickverbindung zum Mammutkopf (\#2);

- die zweite Bewegung ist ein Schritt zurück, der das Kind von dem Schild wegbewegt (\#3, \#4) und der - könnte das Kind schon lesen - eine Lektüre des Schilds verunmöglichte. Diese Bewegung wird begleitet durch eine Zeigegeste. Dass diese Geste auf ein Ziel außerhalb des Mammut-Arrangements 
zeigt, verdeutlicht Nina durch eine schnelle Seitwärtsbewegung, die die gedachte Verlängerung des Zeigefingers am Mammutskelett vorbei führt (\#4).

- Die weiteren Schritte führen Nina an dem Mammut-Skelett vorbei, aber obwohl sie das Geländer berührt (\#5), kann man an der Ausrichtung ihres Unterkörpers, der Richtung ihrer Schritte, der Ausrichtung ihres Kopfs und der Zeigegeste sehen, dass Nina den gemeinsamen Betrachtungsraum verlassen hat.

Zu dem, was ich hier „Arrangement“ genannt habe, zählt nicht nur das Exponat und die erläuternde Objektkennung, wie der folgende Transkriptausschnitt exemplarisch belegt. Zum Arrangement gehört auch ein Ausstellungshilfsmittel wie die Vitrine, die ja sinnvolle Gliederungseinheiten im Ausstellungsraum suggerieren kann (s.o. 4.4.2). Deren Relevanz für die Sichtbarkeit der Beteiligung an einem gemeinsamen Betrachtungsraum kann man in dem folgenden Beispiel sehen („Enten“). Es handelt sich um zwei Mütter („Susanne“, SU, und „Pola“, PO), die mit ihren beiden kleinen Kinder „Julius“ (JU) und „Lars“ („LA“) das Zoologische Museum besuchen. Susanne und Julius haben gerade begonnen, eine Vitrine mit verschiedenen Entenvögeln anzuschauen, Pola und Lars haben die Vitrine noch nicht ganz erreicht.

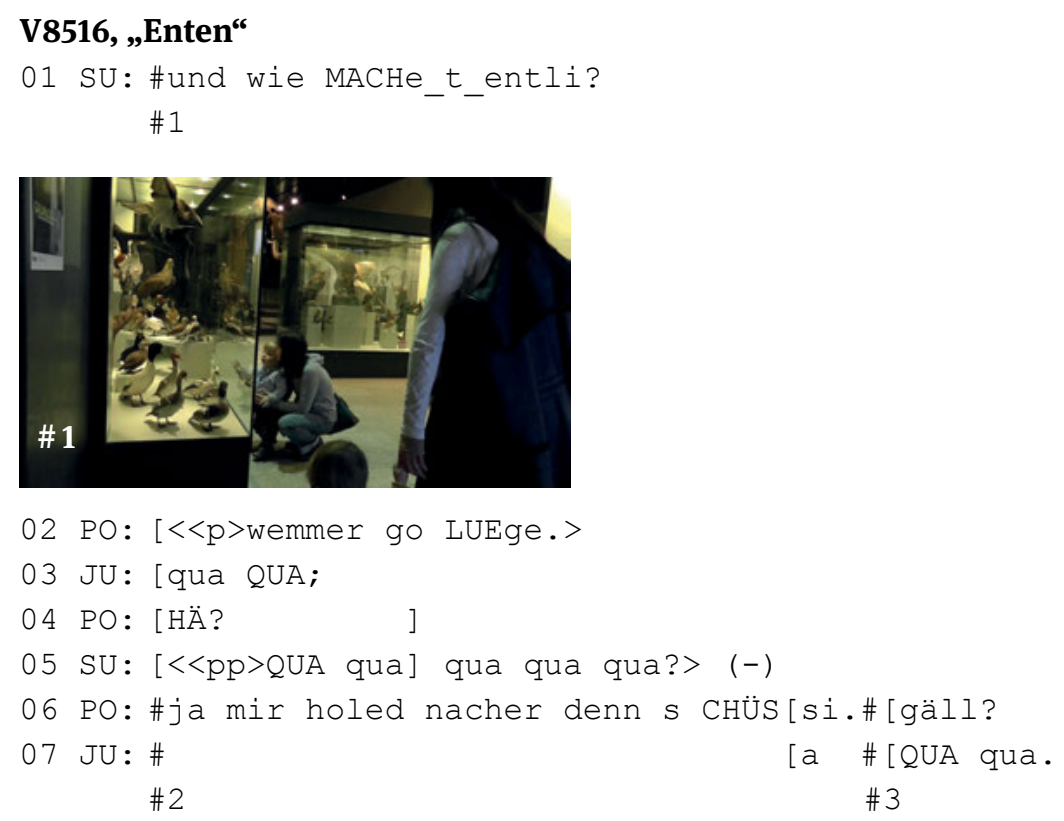



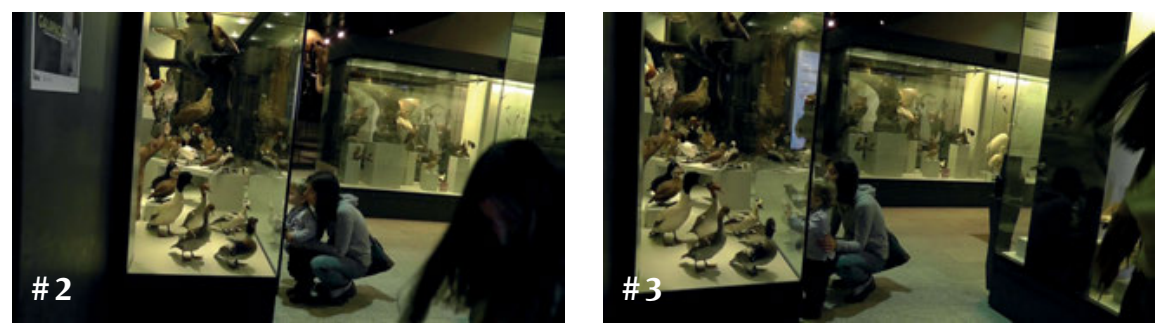

08 SU: ja \#geNAU-\# (-) \#4 \#5
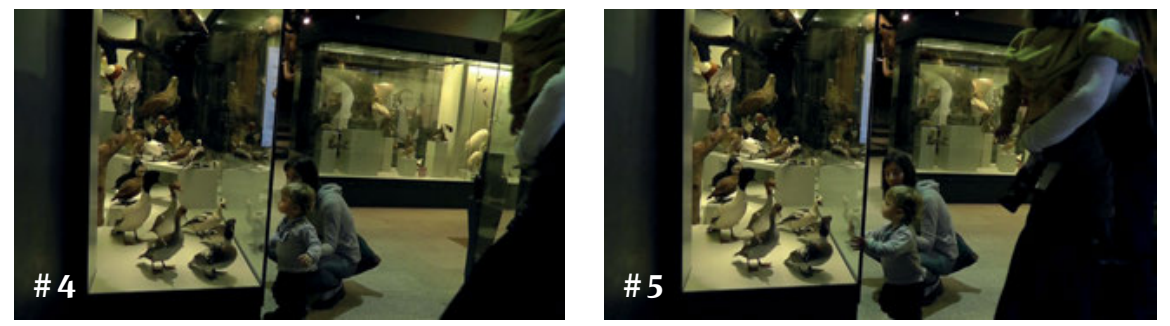

09 PO: xxx xxx tüend mer \#d ENTli alu\#ege? \# 6 \# 7
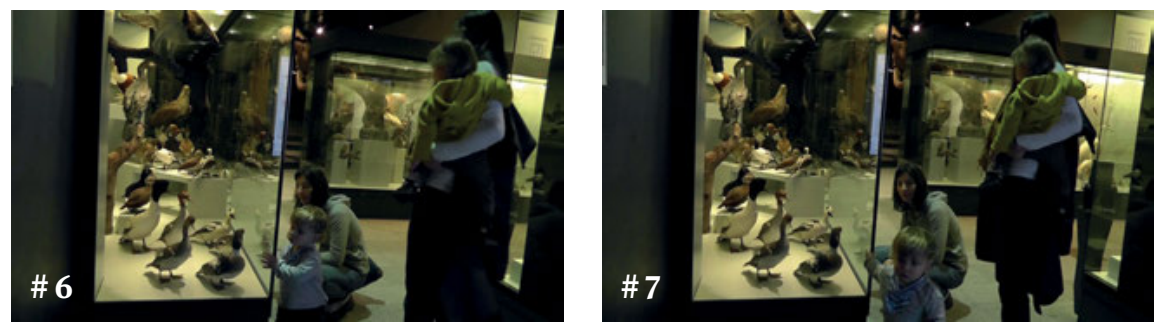

\section{$10 \quad(1.4)$}

11 WO: \#<<leise lachend $>{ }^{\circ} \mathrm{hh}>$ \# 8

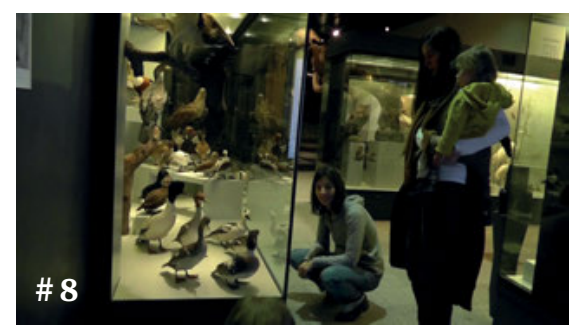

12 SU: <<ruft> JUlius; >

13 PO: wie MAchet denn (d Entn?);

14 WO: ((lacht leise, 3.6 Sek.)) 
$15 \mathrm{SU}$ : HALlo.

16 gAhsch de wider zu de schILDkrot julius. (--)

In diesem Ausschnitt zeigt sich, dass die Vitrine ein ,Hot Spot' innerhalb des musealen Betrachtungsraums ist: Julius' Entfernung von der Vitrine nämlich wird als Aktivität behandelt, die den gemeinsamen musealen Betrachtungsraum infragestellt.

- Zu Beginn des Transkripts versucht Susanne, ihren Sohn Julius dazu zu animieren, Entenlaute nachzuahmen. Susanne hockt zu Beginn dieses Ausschnitts vor der Vitrine und begrenzt die Bewegungsfreiheit ihres Sohns zu beiden Seiten mit ihren vorgestreckten Armen (\#1). Julius führt den Laut zweimal vor. Beim zweiten Mal begleitet er den Entenlaut mit einer Zeigebewegung ins Innere der Vitrine (\#3). Er und seine Mutter befinden sich also in einem gemeinsamen Betrachtungsraum. Welches Objekt genau sich im Zentrum der gemeinsamen Aufmerksamkeit befindet, ist nicht festzustellen. Denn in der Vitrine gibt es eine große Zahl von Entenpräparaten.

- Mit seiner Zeigebewegung beginnt Julius, sich nach rechts zu bewegen (\#4). Seine Mutter lässt ihn aus der Begrenzung ihrer Arme ,entkommen', bis er ans Ende der Vitrine angekommen ist (\#5). Noch immer zeigen Julius' Körper und Kopf in Richtung Vitrine.

- An der Ecke der Vitrine angekommen, wendet er aber plötzlich den Kopf nach links (\#6) und schaut deutlich an der Vitrine vorbei. Sofort wendet die Mutter ihren Kopf vom Vitrineninneren ab und schaut ihrem Sohn hinterher (\#7).

- Julius löst nun auch seine Hand von der Vitrine und geht einen Schritt in den Raum hinein (\#8).

- Mit seiner Ablösung von der Vitrine beginnt seine Mutter zu lächeln, und es ist ein leises, lächelndes Ausatmen des Forschers (WO) zu hören: Beide Erwachsene behandeln Julius' Verhalten als amüsanten Planbruch. Diese Reaktionen setzen praktisch mit seinem Loslassen der Vitrine ein.

Solange sich Julius an der Vitrine entlang hangelt, wird sein Verhalten ganz offensichtlich nicht als problematisch aufgefasst. Das ändert sich genau in dem Moment, in dem er sich von der Vitrinenecke löst. Offenbar setzen die beiden Erwachsenen die Ablösung von der Vitrine mit einem Abbruch des gemeinsamen Schauens gleich.

Nicht nur der Vitrinen-Inhalt sondern auch die Vitrine selbst in ihrer Rolle als sichtbare Gliederungseinheit des dauerkommunikativen Arrangements im Ausstellungsraum, spielen also für die Bewertung eine Rolle, ob ein gemeinsamer Betrachtungsraum geteilt wird oder nicht. Dabei scheint mir in diesem konkreten Fall die Relevanz der Vitrine für den gemeinsamen Betrachtungsraum durch den Umstand gesteigert zu sein, dass sich in der Vitrine zahlreiche Entenpräparate neben- 
einander befinden. Dadurch ist nicht klar, welches Exemplar genau für Susanne und Julius im Zentrum des gemeinsamen Betrachtungsraums steht. Solange man Julius‘ Blick als in die Vitrine gerichtet verstehen kann, solange kann man auch seine Orientierung auf irgendeine Ente unterstellen und so annehmen, dass er seine Aufmerksamkeit auf den gemeinsamen Betrachtungsraum konzentriert.

Zwei Ergebnisse hat die Beschäftigung mit der Auflösung des gemeinsamen Betrachtungsraums erbracht. Zum einen konnten wir sehen, dass der museale Betrachtungsraum zwar stationär ist, aber nicht statisch. Mit anderen Worten: Der Betrachtungsraum wird nicht einmal etabliert und bleibt dann ,einfach“ weiter bestehen. Seine Fortdauer oder Auflösung ergibt sich - wie im Fall des Bewegungsraums - aus den Aktivitäten der Interaktionsbeteiligten, die kontinuierlich und interaktiv aushandeln, wie der museale Betrachtungsraum gestaltet sein soll. Zur Auflösung des Betrachtungsraums reicht ein einfaches Sich-Abwenden eines einzelnen Interaktionsteilnehmers nicht aus. Die Abwendung muss - ähnlich wie es vom Lehn für den Bewegungsraum beschrieben hat (s.o. 5.2) - von den Interaktionspartnern als „first action“ aufgefasst und mit einer „second action“ ,beantwortet' werden: durch ein Akzeptieren oder Ablehnen der Auflösung oder durch das Anbieten eines alternativen Betrachtungsziels (vgl. Patricks aufwändigen Versuch, Nina für den Objekttext zum Mammutexponat zu begeistern).

Während die interaktive Natur des gemeinsamen Wahrnehmungsraums sicher nicht auf den Fall des Museums beschränkt ist, handelt es sich bei dem zweiten hier herausgearbeiteten Aspekt um ein Spezifikum des gemeinsamen Wahrnehmungsraums, wie ihn Besucher für ihren gemeinsamen Besuch herstellen (also den musealen Betrachtungsraum): Im Zentrum des Betrachtungsraums stehen die Exponate (die auch schon als Bewegungsziele in den Blick dieser Untersuchung geraten sind, s.o.), aber auch Erklärungstexte und - sei es als museumsspezifischer Gliederungshinweis (s. 4.4.2), sei es als eine Art metonymischer Ausweitung der Exponate.

\subsubsection{Das genaue Sehen}

Beim Herstellen des musealen Betrachtungsraums geht es nicht um die Herstellung einer einfachen Sichtbarkeit der Exponate. Vielmehr gestalten die Besucher ihren gemeinsamen Wahrnehmungsraum auf eine Weise, die ihnen ein genaues, an der maximalen Aufnahme von Details interessiertes Sehen gestattet. ${ }^{18}$

18 Damit nutzen die Besucher den Raum auf eine Weise, die - wie wir in Kapitel 3 gesehen haben - vom Ausstellungsraum in besonderer Weise wahrscheinlich gemacht wird. 
Der folgende Ausschnitt („Das ist ein Luchs“) zeigt exemplarisch, mit welchem Aufwand Besucher daran arbeiten können, dass ein gemeinsamer Betrachtungsraum hergestellt wird, der ein am Detail interessiertes Sehen der Exponate ermöglicht. Hier organisiert Vater Patrick für seine Tochter Nina die hindernisfreie Wahrnehmung eines Luchs-Exponats.

\section{V8816, „Das ist ein Luchs“}

$01 \mathrm{NI}: \mathrm{mHm}, \quad(--)$

02 un d:̈ dett \#OBbe?

\#1

$03 \quad(-)$

04 PA: dett obbe dä_sch_n LUCHS. (-) \#

$$
\text { \#2 }
$$
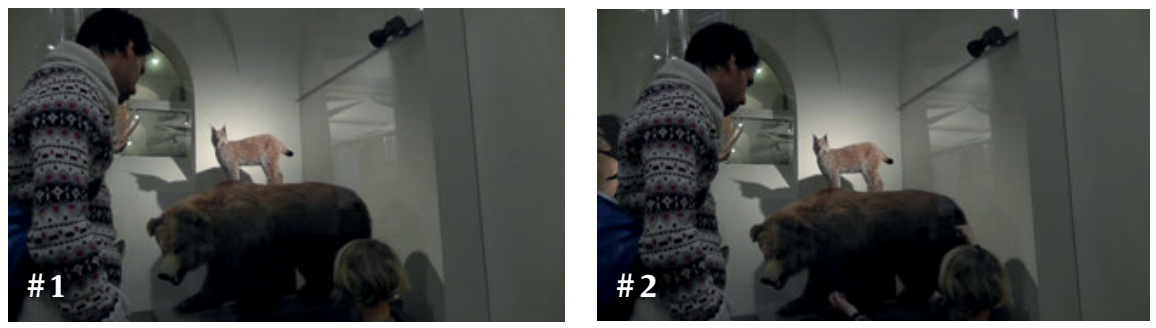

05 NI: $<<$ p >wo händs de GFUNde, >

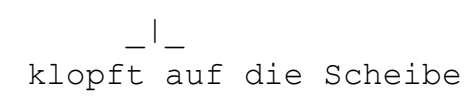

$06 \quad(---) \#(--)$

\#3

07 PA: gsesch \#Ue? (--)

\# 4
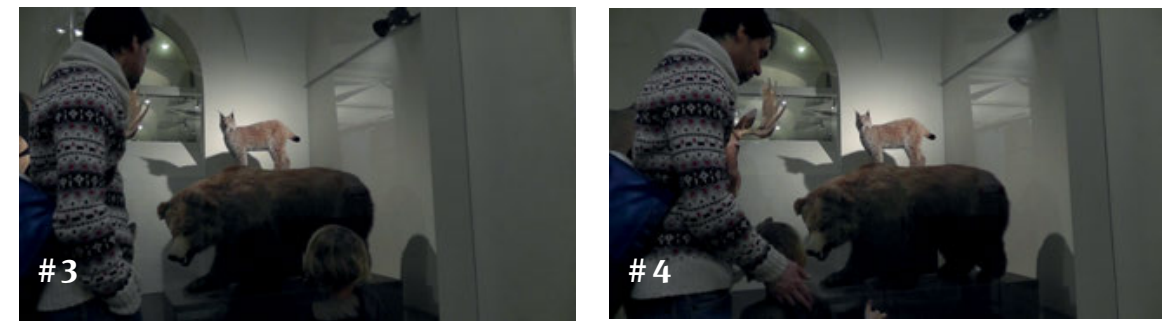

08

$$
\begin{gathered}
\text { gsehsch HINde? (--) \# }(-) \\
\# 5
\end{gathered}
$$

09 NI: NEI? 
10 gseh NÖD.=\#

\# 6
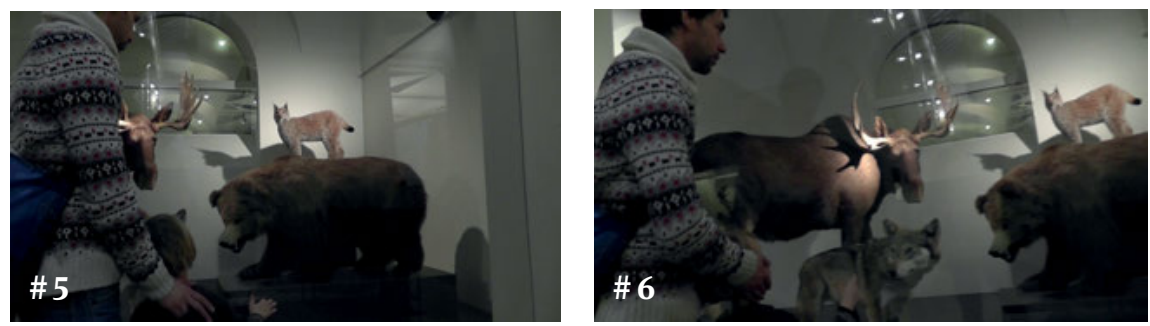

11

$=$ S_SPIE\#gelt.

$\# 7$

12 PA: \#Etz GSEHSCH_es.= \# 8
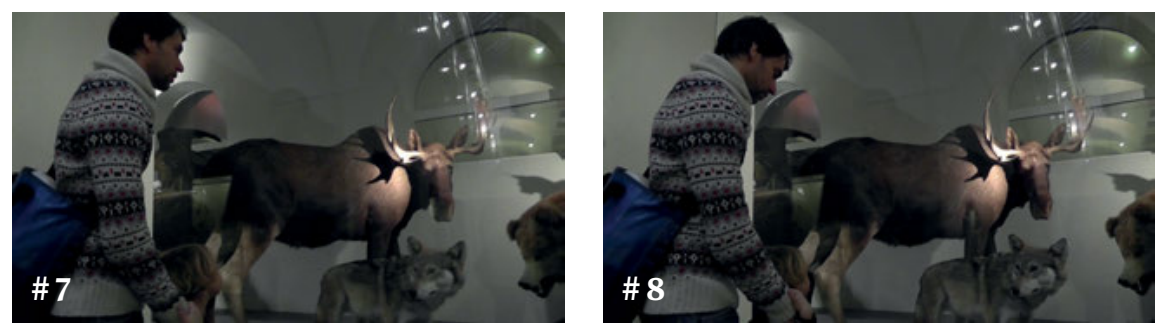

13 =lUg emal was der für luschtigi OHre hät;

In diesem Ausschnitt können wir beobachten, wie der Vater dafür sorgt, dass seine Tochter eine hindernisfreie Perspektive auf das Luchs-Exponat erhält, und wie er Ninas Wahrnehmungsmöglichkeiten kontrolliert, um sicherzustellen, dass sie beide einen geteilten Betrachtungsraum hergestellt haben.

- Nina lenkt die Aufmerksamkeit des Vaters durch das deiktische „dÄ dett OBbe“ (Z. 2) und eine beidhändige Zeigegeste, die den Bär im Vitrinevordergrund wie ,umkurvt‘, (\#1) auf das Luchs-Exponat im Vitrinenhintergrund.

- Mit ihrer Frage „wo händs de GFUNde,“ (Z. 5) beginnt sie sich an der Vitrinenscheibe entlang nach links zu schieben (\#2). Damit nähert sie sich der Position des Vaters, der sie empfängt, indem er seine rechte Hand auf ihren Nacken legt (\#3).

- Ninas Bewegung wird von Patrick als Versuch verstanden, einen geeigneten Betrachtungsstandpunkt zu finden. Denn er fragt sie „gsesch Ue? (--) gsehsch HINde?“ (Z. 7f.). Hierbei geht es nicht allgemein um Ninas Sehfähigkeit, sondern darum, ob sie eine hindernisfreie Sicht auf das Exponat hat, das Patrick mithilfe der Lokaldeiktika genau bestimmt („Ue“ „nach oben“, „HINde“, hinten'). 
- Patrick kontrolliert mit einem Blick herab zu seiner Tochter deren Kopfausrichtung (\#4), wirft dann einen Blick auf den Luchs (\#5), umfasst schließlich Ninas Kopf mit den Händen und richtet ihn auf das Luchs-Exponat aus (\#6).

- Nina macht ihn darauf aufmerksam, dass ihr Standort für die Betrachtung des Luchs-Exponats ungeeignet ist: „NEI? gseh NÖD.==s_SPIEgelt.“(Z.9-11). Wieder geht es - wie häufig in meinem Korpus - nicht um die Sehfähigkeit oder das Sehen allgemein, sondern um das gute, das genaue Sehen.

- Daraufhin überprüft Patrick erneut Ninas Position, indem er zur Tochter und anschließend zum Luchs-Exponat schaut (\#7, \#8) und erklärt „Etz GSEHSCH_ es“ (Z. 12). Damit ist die hindernisfreie Sichtbarkeit des Exponats erreicht und die Arbeit an visuellen Details des Exponats wird aufgenommen: den „luschtigi OHre“ (Z. 13).

Der Ausschnitt „Luchs“ ist in mehrerlei Hinsicht repräsentativ für die Daten meines Korpus.

- Die Besucher arbeiten an der Herstellung einer Perspektive auf die Exponate, die über die bloße Sichtbarkeit hinausgeht. Sie gestalten den musealen Betrachtungsraum vielmehr so, dass er eine präzise Beobachtung ermöglicht, die Details an den Exponaten erkennen lässt.

- Dieser Typ von Kommentar findet sich in meinem Korpus relativ häufig. In allen Fällen geht es um ein museumsspezifisches Sehen, das durch Hindernisse unterschiedlicher Art verhindert wird: eine Person, die zwischen dem Besucher und einem interessierenden Exponat steht, ein zu hoch angebrachtes Exponat, ein Mikroskop, das nur von einer Person benutzt werden kann, während die anderen nur seitlich auf den Mikroskopträger schauen können usw.

- Alle Beteiligten sind aktiv an der Herstellung des gemeinsamen Betrachtungsraums beteiligt. Für Besuchergruppen mit Kind heißt das: Die Erarbeitung des musealen Betrachtungsraums geht nicht nur von Erwachsenen aus. Selbst ganz kleine Kinder sind schon aktiv an seiner Hervorbringung beteiligt. Auf Erwachsene beschränkt sind in meinem Korpus aber körperliche ,Zwangsmaßnahmen' wie das hier beobachtete Drehen des Kopfes.

- Schließlich stellt der museale Betrachtungsraum die Voraussetzungen zur Verfügung, die nötig sind, um über die Exponate gemeinsam Wissen zu konstruieren. In diesem Sinne ist er, wie wir in 5.4 im Detail sehen werden, auf die Herstellung des musealen Handlungsraums bezogen.

Im nächsten Analyseschritt möchte ich der Frage nachgehen, wie die Bewegungen der Besucher in der „Verweilzone“ vor den Exponaten durch ihre Orientierung auf die Optimierung der Sichtbarkeit der Exponate bestimmt werden. Dieser 
Aspekt wird im folgenden Ausschnitt „Lupfen“ besonders gut sichtbar, den ich aus Darstellungsgründen in drei Teile unterteilt habe.

Wir sehen einen Großvater („Otto“, OT), der mit seinem anderthalbjährigen Enkel „Samuel“ (SA) auf das große, freistehende Mammutskelett zuläuft, das wir schon aus dem Video mit Patrick und Nina kennen.

\section{V8158, „Lupfen“ 1 . Teil}

01 OT: JETZ gömmer de gross\# go <<lachend>aluege, >
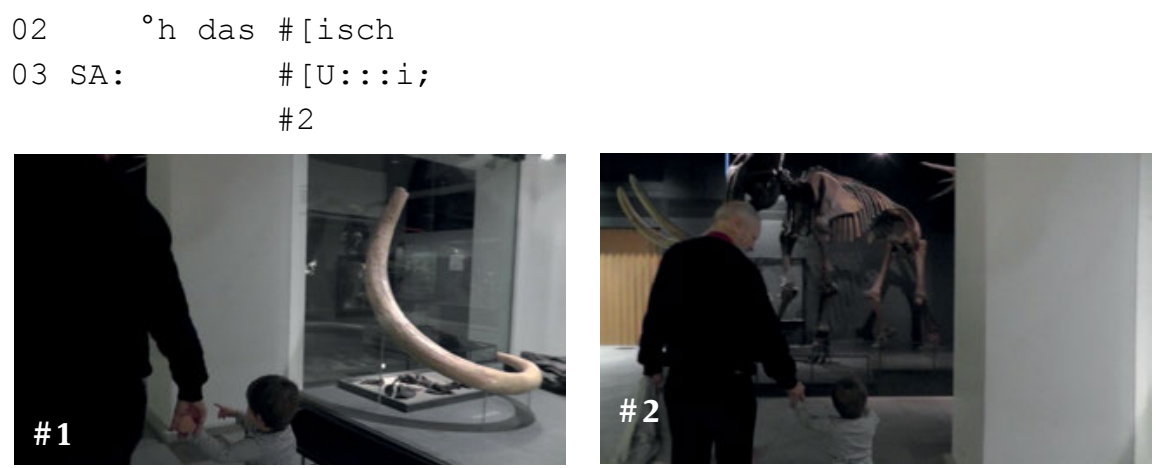

04 OT: das isch Ungehür; =hä?

$05 \quad(2.0)$

$06 \mathrm{SA}:<<\mathrm{p}>\left(\mathrm{m}_{-} \mathrm{Hm}\right),>$

$07 \quad(1.0)$

08 OT: \#Ch_tÜe di mal chli lUpfe dänn \#º GSEHSCH_es nacher besser

\#3

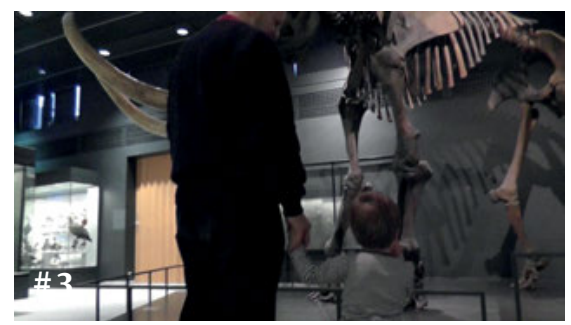

\# 4

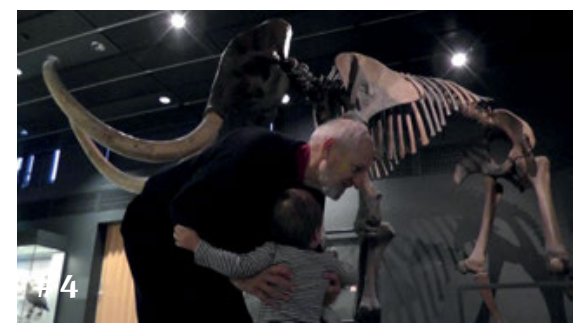

09 OT: in e Andere pers\#[pektive,

$10 \mathrm{SA}: \quad$ \#[ (UI);

\# 5 


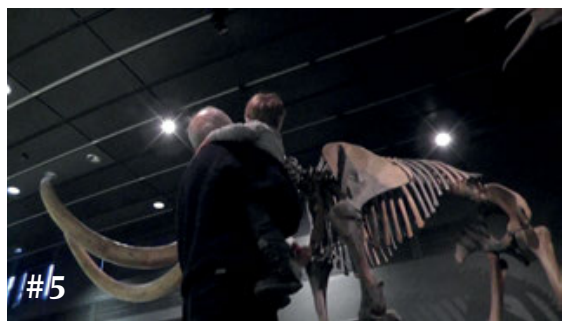

Ottos Bewegungen sind hier sicht- und hörbar durch das Bestreben motiviert, seinem Enkel möglichst detailreiche Ansichten des Mammuts zu ermöglichen.

- Zu Beginn des Segments sehen wir Otto und Samuel auf dem Weg zum Mammutskelett. Schon längere Zeit hat Samuel erfolglos versucht, seinen Opa zur Annäherung an das Mammutexponat zu bewegen (vgl. Samuels Zeigegeste in \#1); nun gibt Otto dem energischen Drängen des Kindes nach und akzeptiert mit dem Kommentar „JETZ gömmer de grOss go <<lachend>aluege,>“ (Z. 01) das freistehende Mammutskelett als nächstes gemeinsames Bewegungs- und Betrachtungsziel.

- Noch während sich die beiden dem Mammut nähern, etablieren sie einen gemeinsamen Wahrnehmungsraum, in dessen Zentrum das Mammut steht: Otto nimmt deiktisch auf das Mammut Bezug (, ${ }^{\circ} \mathrm{h}$ das isch das isch Ungehür;=hä?““, Z. 4) und Samuel zeigt seine Ausrichtung auf das Mammut mit seinem Blick und einer deiktischen Geste (\#2). Beide treten so nahe an das Mammutexponat heran, wie es ihnen durch das Podest und das Geländer ermöglicht wird (\#3). Hier könnte die Betrachtung des Mammuts beginnen, der gemeinsame Betrachtungsraum ist etabliert.

- Doch sieht der Großvater den Standpunkt des Kinds offenbar nicht als optimal an. Mit dem Kommentar „ch_tÜe di mal chli lUpfe dänn º GSEHSCH_es nacher besser in e Andere perschpektive,“ (,ich hebe dich schnell hoch...', Z. 8f.) hebt er das Kind hoch (\#4), womit er dessen Kopf auf die Höhe seines eigenen Kopfes bringt (\#5). Die einfache Sichtbarkeit des Mammuts ist ihm also nicht genug, er schafft dem Kind durch das Hochnehmen - wie er selbst erläutert - eine Perspektive, die nicht nur eine andere, sondern eben eine bessere Sicht auf das Mammut ermöglicht.

Es zeigt sich hier: Der gemeinsame Wahrnehmungsraum soll nicht nur ein einfaches Sehen, sondern ein ,verbessertes“ Sehen ermöglichen. Für dessen Zustandekommen ist Otto bereit, einigen körperlichen Einsatz zu leisten: vgl. sein hörbares Einatmen beim Hochnehmen des Kindes. Die Orientierung am genauen Sehen ist auch im 2. Teil des Ausschnitts „Lupfen“ zu beobachten, der etwa 20 Sekunden nach Ende des letzten Transkripts einsetzt. 


\section{V8158, „Lupfen“ 2. Teil}

01 OT: [LUEG emal ] hier LUEG emal (-)\# die BEI;

$02 \mathrm{SA}:[\mathrm{XXX}$ XXX $\mathrm{XXX}]$

\#

$\# 1$

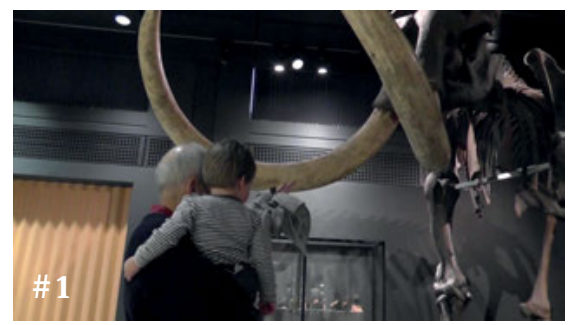

03 OT: $\langle<\mathrm{p}>($ GÖMmer mal) $-(-) \quad($ LUG emal) $->$

$04 \quad(4.6)$

05 OT: gömmer mal vo \#DEre siite,\#
\#2
\# 3
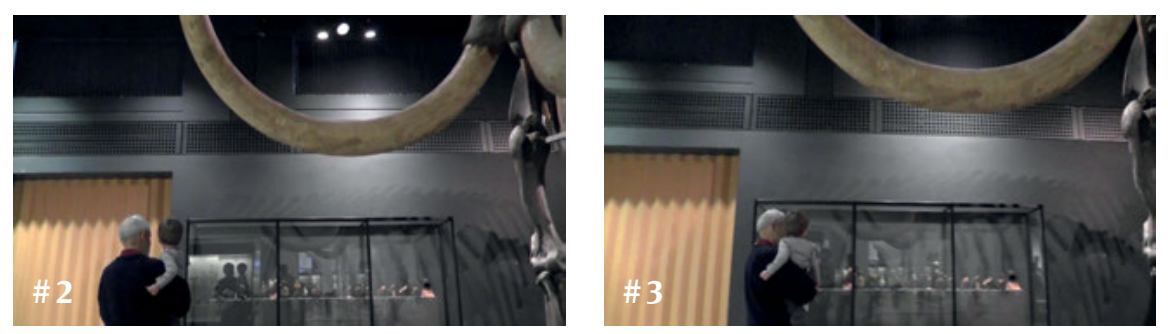

06

$$
<<\text { ) }
$$
\#5
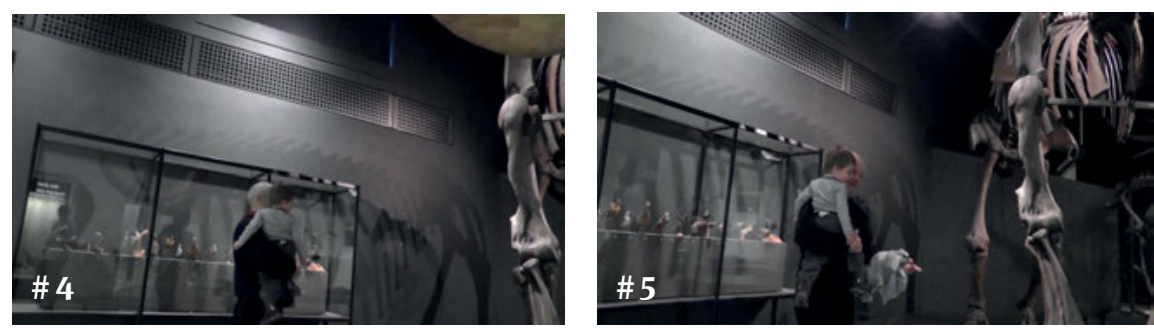


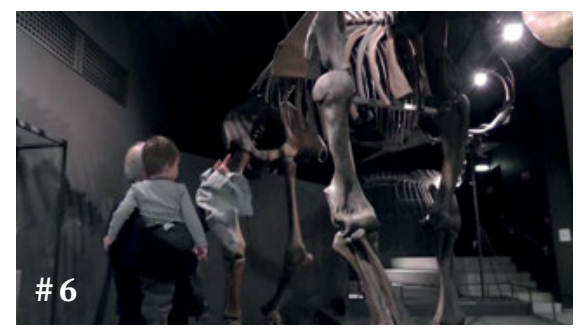

Mit Beginn des Transkripts stehen Otto und Samuel zwischen den Stoßzähnen des Mammuts (\#1).

- Otto zeigt auf einen weiter entfernten Teil des Mammutskeletts, den er verbal („die BEI“) sowie durch eine ikonische Geste als die Beine des Mammuts identifiziert (die Geste zeigt Lage und Länge der Beine an). Die Mammutbeine befinden sich im gemeinsamen Wahrnehmungsraum der beiden Besucher.

- Doch reicht Otto offensichtlich die bloße Sichtbarkeit der Beine nicht aus. Er geht um das Mammut herum, bis er direkt neben den Vorderläufen des Mammuts zum Stehen kommt (\#2-\#5), wobei er seinen Laufweg so nah an das Mammut verlegt, wie es das Podest, auf dem das Skelett aufgestellt ist, eben gestattet.

- An dem Punkt der größtmöglichen Annäherung an das Mammutbein zeigt Otto zum zweiten Mal auf das Bein. Jetzt schreibt er ihm eine Eigenschaft zu: seine Größe (\#6).

Die Tatsache, dass Otto seinen Enkel so nah wie möglich an das Mammutbein heranbringt, bevor er seinen Enkel auf die Größe des Mammutbeins aufmerksam macht, obgleich diese Eigenschaft auch vom vorigen Standpunkt aus hätte beobachtet werden können, scheint mir ein starkes Argument dafür zu sein, dass es zu Ottos ,verborgener Agenda' gehört, seinem Enkel diesen Aspekt der Betrachtung von Exponaten zu vermitteln: nämlich dass Exponate aus möglichst großer Nähe $\mathrm{zu}$ betrachten sind.

In den zwei Teilen des Ausschnitts „Lupfen“ konnten wir sehen, dass die Bewegungen der Besucher durch die Verweilzone des Mammut-Exponats auf das Erreichen von Standorten gerichtet sind, von denen aus eine möglichst gute Wahrnehmung der thematisierten Teile oder Bereiche des Exponats möglich ist. Nicht nur rechtfertigt Otto alle seine Positionsänderungen in der Verweilzone um das Exponat mit dem Vorhandensein neuer Möglichkeiten des Schauens (jede Bewegung wird mit dem Hinweis auf das Schauen eingeleitet). Auch die Wege um das Exponat herum gestaltet Otto so, dass der Abstand zum Exponat möglichst gering ist, was dem schauenden Samuel durchgängig den detailhaltigsten Blick auf das Exponat ermöglicht. Und Ottos Wege enden möglichst nahe an dem 
thematisierten Bestandteil des Exponats, selbst wenn das für die von ihm verbal hervorgehobenen Merkmale des Exponats (etwa die Größe der Füße) nicht immer nötig gewesen wäre. Bei all diesen Bewegungen trägt er Samuel auf dem Arm. Dies bringt Samuel näher an das hoch aufragende Exponat heran, macht es Otto aber gleichzeitig leichter, den Betrachtungsprozess seines Enkels zu kontrollieren. Wieder lässt sich hier also verfolgen, wie Erwachsene sicherzustellen versuchen, dass die begleitenden Kinder bestimmte museumsspezifische Wahrnehmungen machen.

Neben so auffälligen Formen der Orientierung am genauen Sehen gibt es in meinem Material viele beiläufigere Formen, die die Relevanz des genauen Sehens sichtbar werden lassen. Etwa wenn Besucher sich den Exponaten in einer Vitrine so weit wie irgend möglich zu nähern versuchen und sich dabei an das Vitrinenglas ,schmiegen' (Abb. 5-25), wenn sie sich auffällig und mühevoll vorbeugen und dabei ihre Augen beinahe an das Vitrinenglas drücken (Abb. 5-26) oder wenn Eltern ihre Kinder so hochheben, dass sie den von dem Exponat suggerierten Betrachtungsstandpunkt einnehmen können (in Abb. 5-27 ist es die Aufsicht auf das Schlangenskelett, die durch die Form der Vitrine nahegelegt wird).

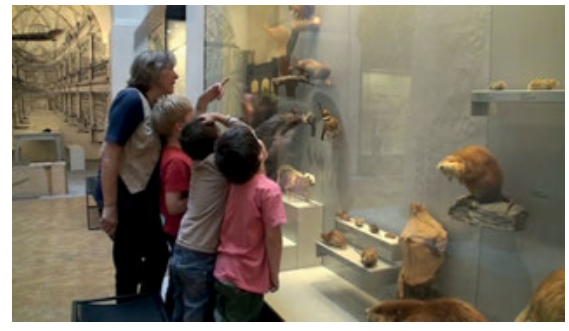

Abb. 5-25

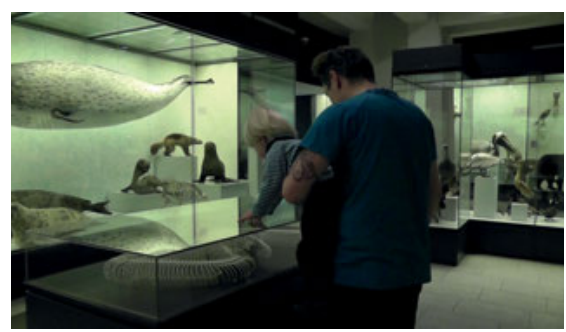

Abb. 5-27

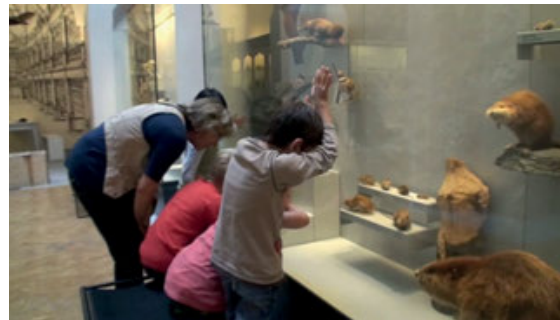

Abb. 5-26 


\subsubsection{Fazit: der museale Betrachtungsraum}

In diesem Analyseabschnitt haben wir gesehen, wie die Museumsbesucher in ihrer Interaktion einen gemeinsamen Wahrnehmungsraum hervorbringen und seine Aufrechterhaltung bzw. Auflösung interaktiv aushandeln. Die Aufgabe der Ko-Orientierung wird dabei nicht nur durch körperliche Ressourcen bearbeitet (konkret: durch die Ausrichtung des Körpers und speziell des Oberkörpers und Kopfs, durch Blickrichtung oder Zeigegesten), sondern auch durch Sprache: In dem zuerst untersuchten Ausschnitt (,Riesengroßes Tier“, s.o.) steht die sprachliche Definition des Betrachtungsziels gleich am Anfang der Ko-Orientierungsbemühungen der Interaktionsbeteiligten, und die Verwendung sprachlicher Deiktika zeigt den Moment an, in dem die Beteiligten wechselseitig davon ausgehen, dass sie einen gemeinsamen Wahrnehmungsraum erzeugt haben.

Der von den Besuchern hergestellte Wahrnehmungsraum weist einige charakteristische Besonderheiten auf, die seine Musterhaftigkeit als musealer Betrachtungsraum ausmachen. Diesen Besonderheiten sind wir in 5.3.3 und 5.3.4 nachgegangen.

So habe ich zeigen können, dass die Besucher bei ihrem gemeinsamen Gang durch die Ausstellung typischerweise einen visuellen Wahrnehmungsraum konstruieren, dessen Aufmerksamkeitszentrum die Exponate sind. Anstatt einen von ihren Körpern ,umstandenen“ „o-space“ herzustellen, in den hinein sie sprechen, gestikulieren und in dem sie ihre gemeinsame Aufmerksamkeit fokussieren (Ciolek/ Kendon 1980: 258), richtet sich die visuelle Aufmerksamkeit der Museumsbesucher auf das semiotische Angebot der Ausstellung. Die Besucherkörper wie ihre Gesten sind auf diesen ,exzentrischen` Betrachtungsraum hin ausgerichtet, während sich die Besucher selbst über weite Strecken nur über die periphere Sicht wahrnehmen - man kann sagen, dass der Betrachtungsraum die visuellen Wahrnehmungen der Besucher im Ausstellungsraum gleichsam monopolisiert.

Im Zentrum dieses exzentrischen Wahrnehmungsraums befinden sich, wie ich anhand einer Reihe von Ausschnitt nachgewiesen habe, relevante Elemente des multimodalen kommunikativen Angebots der Ausstellung: hauptsächlich Exponate, aber auch Texte, Vitrinen und dergleichen mehr. Die Form und Ausrichtung des speziellen, auf die Praxis des gemeinsamen Museumsbesuchs zugeschnittenen Wahrnehmungsraums wird durch die Möglichkeiten definiert, die relevanten Elemente der Ausstellung detailorientiert zu betrachten. In dieser Orientierung am genauen Sehen, an der detailorientierten Betrachtung der Exponate, liegt ein weiteres Spezifikum des geteilten Wahrnehmungsraums, wie er für die Zwecke des gemeinsamen Museumsbesuchs hergestellt wird. Für jenen reicht es nicht aus, dass zwei Personen einfach dasselbe sehen. Vielmehr geht es 
darum, eine gemeinsame Sicht auf die Exponate hervorzubringen, die ein intensives, am Detail interessiertes visuelles Studium der Exponate gestattet.

Damit sind die musterhaften Elemente des musealen Betrachtungsraums benannt: Es handelt sich beim musealen Betrachtungsraum um die spezifische Ausprägung eines interaktiv hergestellten gemeinsamen Wahrnehmungsraums, der nicht auf die gegenseitige Wahrnehmung der Interaktionspartner, sondern auf das intensive, möglichst kein Detail ,verschenkende“ Betrachten von Exponaten (und anderen Elementen des multimodalen Kommunikationsangebots der Ausstellung) ausgerichtet ist. Dieser museale Betrachtungsraum ist derart musterhaft, dass wir in den Personen, die auf den Standbildern abgebildet sind, sofort und unzweifelhaft Museumsbesucher bei der Betrachtung von Exponaten erkennen.

In den in diesem Abschnitt untersuchten Ausschnitten konnten wir sehen, wie Erwachsene mit einigem Aufwand und teilweise mit leichtem körperlichen Zwang (Hochheben, Drehen des Kopfes) versuchen, ihren Kindern oder Enkelkindern die angemessene Beteiligung am musealen Betrachtungsraum zu vermitteln, indem sie deren visuelle Orientierung auf die Exponate überprüfen und korrigieren. Die Vermittlung eines museumsadäquaten Betrachtens gehört also offensichtlich ebenso sehr zur Vermittlung der angemessenen Ausstellungsnutzung wie die Vermittlung der angemessenen Bewegungen, die wir in 5.2 beobachtet haben. Im folgenden Analyseabschnitt (5.4) werden wir sehen, dass der von den Besuchern ko-konstruierte Betrachtungsraum die Voraussetzungen schafft für die weitere Beschäftigung mit den Exponaten im Sinne einer lokalen Konstruktion von Wissen.

\subsection{Die Herstellung des musealen Handlungsraums}

\subsubsection{Ausgangspunkt}

Im vorigen Analyseabschnitt haben wir gesehen, wie die Museumsbesucher im Lauf ihres Gangs durch die Ausstellung immer wieder gemeinsame visuelle Wahrnehmungsräume herstellen, in deren Zentrum sich die Exponate der Ausstellung befinden. Die Frage, wofür die Besucher diese gemeinsamen Wahrnehmungsräume im Lauf ihres Gangs durch das Museum herstellen, ist allerdings noch nicht mit ausreichender Tiefe behandelt worden. Zwar ist das Betrachten der Exponate eine Aktivität, die - wie wir in Kapitel 3 gesehen haben durch den Ausstellungsraum besonders prominent gemacht wird und die daher hochgradig erwartbar ist. Und dennoch vermuten wir aufgrund eigener Besuchserfahrungen, dass sich die Aktivitäten der Museumsbesucher nicht im 
Betrachten der Exponate erschöpfen. Im folgenden Analyseabschnitt werde ich rekonstruieren, welche Aktivitäten die soziale Praxis des gemeinsamen Museumsbesuchs ausmachen.

Bei meinen Analysen kann ich erneut auf eine Reihe von Arbeiten zurückgreifen, die im Umfeld von Christian Heath am Londoner Kings' College entstanden sind und auf die weiter oben mehrfach hingewiesen wurde (Heath/vom Lehn 2001, Heath et al. 2002, Heath/vom Lehn 2004, Heath/vom Lehn/Osborne 2005, vom Lehn/Heath 2007a, 2013, 2016). Für die Untersuchung der Aktivitäten, die die soziale Praxis des Museumsbesuchs ausmachen, sind sie von besonderem Interesse, da sie materialbasiert herausarbeiten, wie in der Interaktion Sprechen, körperliche Ressourcen und Artefakte konkret zusammenspielen. Dabei können die Autoren zeigen, wie Exponate im Lauf der Interaktion mit Bedeutungen versehen und wie sie mit Handlungen gleichsam , aufgeladen' werden können (s. besonders Hindmarsh/Heath 2003), sodass sie eine ,Ressource‘ für die weitere Interaktion werden können. Die hier entstandenen Studien zu Museums- und Galeriebesuchen zeigen: „the experience of exhibits and exhibitions rests upon a complex social and interactional organisation“ (Heath/vom Lehn 2001). Konkret heißt das beispielsweise, dass die Besucher die Erfahrungen der anderen, die Art und Weise, wie jene ein Exponat sehen und verstehen, mitformen (Heath/vom Lehn 2004: 46).

Einen weiteren relevanten Bezugspunkt stellen Forschungen zum Lernen im Museum dar, die mit Gesprächsdaten (sowie bisweilen mit Video) arbeiten. Viele davon widmen sich der Vermittlung von naturwissenschaftlichem Wissen und naturwissenschaftlichen Methoden in Museen und Science Centres (s. etwa Allen 2007, Ash 2007 oder Callanan/Valle/Azmitia 2007, Atkins et al. 2009 oder Crowley et al. 2001). Hinzu kommen Untersuchungen aus dem Paradigma der „conversational elaboration“, die Lernen operationalisieren als eine Erweiterung der Fähigkeit, sich über Themen der Ausstellung zu äußern (Leinhardt/ Crowley 1998 und s.o. 3.1). Schließlich sind für die Untersuchung des Handlungsraums Arbeiten einschlägig, die empiriebasiert zu bestimmen versuchen, welche Lerneffekte vom Museum zu erwarten sind (vgl. Hooper-Greenhill 2002: 11-17 oder National Council 2009 zu „learning outcomes“; Schrader/Stadler/ Körber 2008, Noschka-Roos/Lewalter 2013 zum informellen Lernen; Allen/ Peterman 2019, Serrell 2020 zur Perspektive der Ausstellungsevaluation).

Bei meinen Analysen wird ein Aspekt im Vordergrund stehen, der sich schon in Kapitel 3 als für die von mir untersuchten Ausstellungen besonders relevant erwiesen hat: der Aspekt der Vermittlung von Wissen. Anders als in Kapitel 3.7 betrachte ich die Wissenskommunikation aber nicht vom Raum und dem von ihm Suggerierten aus, sondern von der Interaktion der Besucher. Ich frage, wie die Besucher das im Raum arrangierte multimodale Kommunikationsangebot für ihre lokale Herstellung von geteiltem Wissen nutzen. 
Meine Auffassung von „Wissen“ schließt, wie in Kapitel 3 ausgeführt, an konversationsanalytisch inspirierten Arbeiten zur lokalen Genese von Wissen an (Dausendschön-Gay/Domke/Ohlhus 2010, Bergmann/Quasthoff 2010, Stivers/ Mondada/Steensig 2011, Deppermann 2018). Aus dieser Perspektive ist Wissen keine individualpsychologische Größe, sondern Gegenstand und Resultat einer schrittweisen, gemeinsamen Hervorbringung in der Interaktion (,lokale Generierung'). Als Wissen wird das verstanden, was sich die Interaktionspartner als geteilte Gewissheit anzeigen. Diese Sichtweise lenkt die Aufmerksamkeit weg von der praktischen Frage, was ein Besucher im Lauf seines Museumsbesuchs Neues gelernt hat, hin zu der Rekonstruktion der kommunikativen Verfahren, auf die die Besucher zurückgreifen, um sich in der Ausstellung Fachwissen zu erarbeiten und wie sie sich die Geteiltheit der erarbeiteten Überzeugungen signalisieren. Das erlaubt es, die Frage zu stellen, wie es möglich ist, ein Arrangement im physischen Raum - als das wir die Ausstellung ja in Kapitel 3 beschrieben haben - zu nutzen, um sich mit ihrer Hilfe Wissen zu erarbeiten.

Im folgenden Abschnitt möchte ich zunächst der Frage nachgehen, wie sich Gespräche in der Museumsausstellung als Fälle von Wissenskommunikation ausweisen, also wie sich die Besucher gegenseitig signalisieren, dass sie dabei sind, sich Wissen zum Ausstellungsthema zu erarbeiten (5.4.2). Anhand einiger längerer Transkriptausschnitte werde ich die Charakteristika der musealen Wissenskonstruktion rekonstruieren. Besonders herausstellen werde ich den charakteristischen Raum- und Objektbezug der Wissenskonstruktion in der Museumsausstellung: Die Besucher nutzen Elemente des Raums, speziell die Exponate, als Zeichen und schließen von individuellen Merkmalen der Exponate auf allgemeine Merkmale der repräsentierten Klasse von Tieren. Dabei bringen die Besucher auch Vorwissen in die museale Wissenskonstruktion mit ein, das sie dann an dem in der Ausstellung Wahrnehmbaren verankern. In 5.4.3 werde ich untersuchen, wie die Besucher den Handlungsraum konstruieren, der ihnen die museumsspezifische Wissenskommunikation ermöglicht. Speziell geht es um die Frage, wie im Museumsbesuch Handlungs- und Betrachtungsraum miteinander zusammenhängen. Ich werde materialgestützt dafür argumentieren, dass es bei aller Relevanz, die dem Betrachten beim Museumsbesuch zukommt, dennoch nicht gerechtfertigt ist, Betrachtungs- und Handlungsraum in eins zu setzen. Abschließend werde ich mich in Abschnitt 5.4.4 mit der Rolle auseinandersetzen, die Spaß und Spiel für die Rekonstruktion der hier untersuchten sozialen Praxis einnehmen. Dabei werde ich zeigen, dass selbst Spiel und Spaß von den Besuchern für die Zwecke der Wissenskonstruktion genutzt werden können, dass im Spiel aber auch ein subversives Potenzial enthalten ist, das die Durchführung des gemeinsamen Museumsbesuchs bedrohen kann. 


\subsubsection{Die interaktive Konstruktion von Wissen in der Museumsausstellung}

Welche gemeinsamen Aktivitäten führen die Museumsbesucher im Ausstellungsraum aus und worin liegt deren Museumsspezifik? Diese Frage möchte ich anhand eines Videos in Angriff nehmen, mit dem wir uns schon mehrfach beschäftigt haben. Das Video zeigt Vater Patrick mit seiner Tochter Nina beim Besuch des Zoologischen Museums.

In dem folgenden Ausschnitt (,Bartgeier“) befinden sich Patrick und Nina vor einer Vitrine zu Tieren, die in der Schweiz ausgestorben sind. Das Transkript beginnt mit einem Beitrag Ninas, die ihren Vater auf ein Bartgeier-Exponat aufmerksam macht.

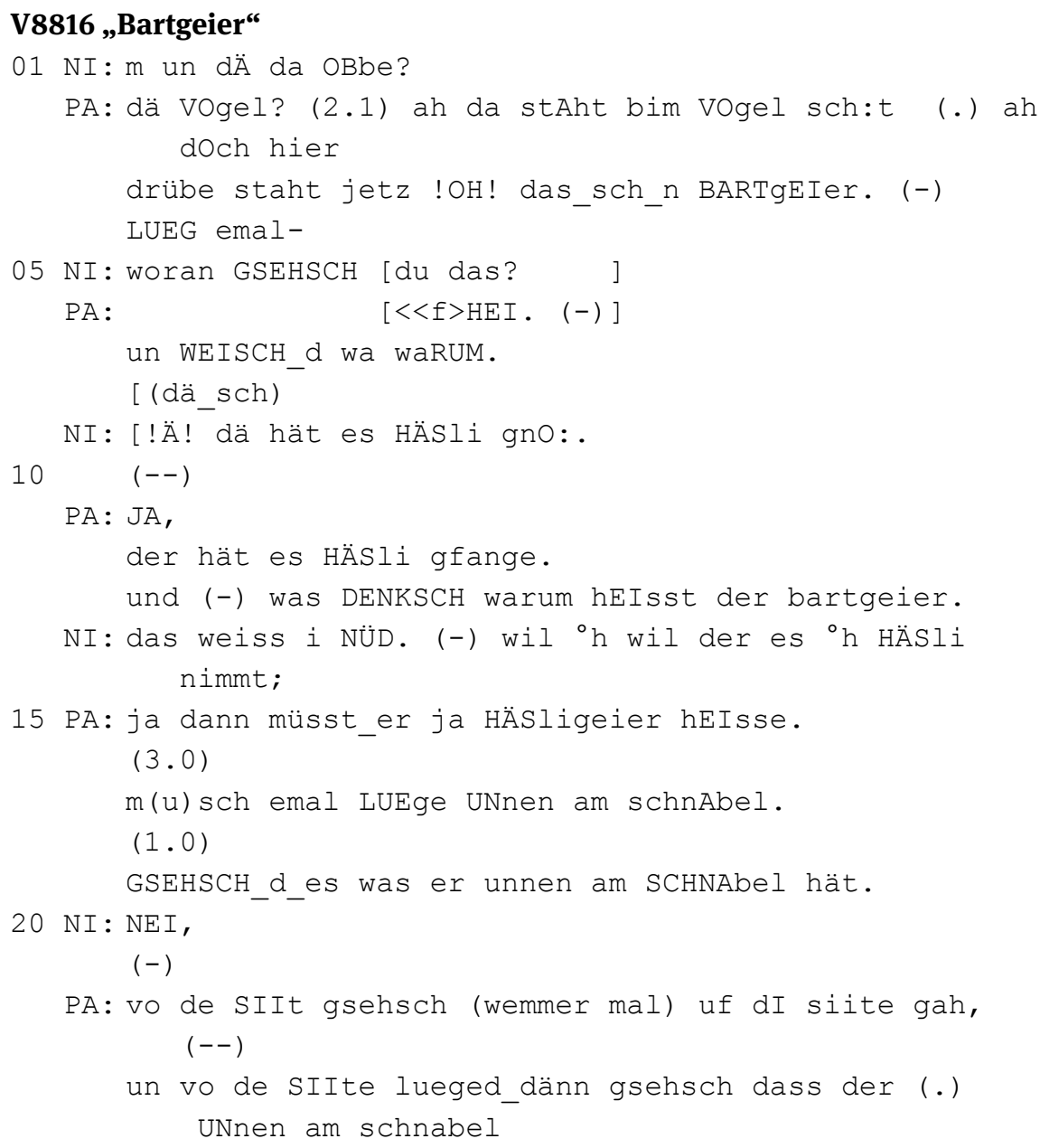


hät der en BART.

$25 \quad(1.6)$

und darum heisst er BARTgeier. (---) und DÄ:,

NI: tUEt, (-) tuet_HÄSli fange.

$\mathrm{PA}$ : der tuet_HÄSli fange, =

$=$ ' JA:,

30 NI: und wo hänt sie DÄ gfunde?

PA: dë hät mä (.) i de SCHWIIZ gfunde, (--)

aber nöd da in ZÜri? (-)

so:nde:rn (-) im WALlis.

$(--)$

35 NI: und s das hësli wo hänt s DAS gfun[de?

PA:

[wo_s_HÄSIi

gfunde hänt das

staht jetz nö:d;

(2.0)

NI: (wo se s) HÄSli gfunde hänt;

$40 \quad(1.8)$

dÄnn (xxx $x x x)$ isch_s häsli AU tot.

PA: s hësli isch AU tot.=ja,

$(--)$

das FRISST der ebbe;

45 der (-) der bartgeier der schnappt sich sones HëSli,

$(1.0)$

NI : mi_m SCHNAbel-

PA: mit em schnabel !UND! mit_e CHRALle.

(2.4)

50 NI: (dä) chann so SCHNÄLL flüge; =

PA: =dä cha SCHNÄLL flüge.=hä,

Die Aktivität, der Patrick und Nina hier gemeinsam nachgehen, erschöpft sich nicht im genauen Ansehen des Bartgeier-Exponats. In diesem Ausschnitt geht es mindestens ebenso sehr um die Erarbeitung von geteiltem Wissen, genauer: von geteiltem Wissen zum Sachgebiet der Zoologie.

Auf welche Indizien kann sich diese Behauptung stützen? Das klarste Indiz für die Relevanz des Wissens in diesem Ausschnitt ist sicherlich die explizite Thematisierung von Wissen und Nichtwissen durch die Interaktionsbeteiligten (,WEISCH_d waRUM.“, Z. 7; „das weiss i NÜD.“ (Z. 14). 
Ein zweites Indiz für die Bedeutsamkeit von Wissen in diesem Gespräch ist die durchgehende Dominanz der kommunikativen Funktion der ,Darstellung (Hausendorf/Kesselheim 2008: 145-148) und damit die Ausrichtung des Gesprächs auf die Leitunterscheidung ,wahr vs. falsch', die als typische Merkmale der Wissen(schaft)skommunikation beschrieben worden sind (s. dazu beispielsweise Steinhoff 2007: 9-13). Auffällig ist hier ein erhöhter Anspruch an das Zutreffen und die Genauigkeit der Behauptungen im Gespräch. Das zeigt sich etwa darin, dass die Aussagen eines der Gesprächspartner immer wieder vom anderen explizit ratizifiert werden (,JA,“, Z. 11, „’JA:,“, Z. 29, s hÄsli isch AU tot.=ja,“ Z. 42, „dä cha SCHNÄLL flüge.=hä,“, Z. 51) oder dass hier mehrmals Aussagen vom Sprecher selbst oder seinem Gegenüber im Nachgang präziser gefasst werden (vgl. „i de SCHWIIZ [...] aber nöd da in ZÜri? (-) so:nde:rn (-) im WALlis.“, Z. 31-33; „mi_m SCHNAbel-“ - „mit em schnabel !UND! mit_e CHRALle.“, Z. 47f.). Hierzu passt, dass Patrick und Nina besondere Anforderungen an die konversationelle Stützung ihrer Tatsachenbehauptungen stellen, etwa wenn sich Nina nicht mit der Kategorisierung des Exponats als Bartgeier zufrieden gibt, sondern mit der Nachfrage „woran GSEHSCH du das?“ (Z. 05) einen Beleg für die Behauptung des Vaters einfordert.

Ein drittes Indiz dafür, dass Patrick und Nina sich in dem obigen Ausschnitt Wissen erarbeiten, ist das häufige Vorkommen argumentativer Strukturen, speziell: das häufige Herstellen kausaler Relationen. Allein im Zusammenhang mit dem ,Hasenfang، findet man die folgenden:

- Patrick fordert Nina dazu auf, eine Hypothese zum Motiv der Namensgebung zu entwickeln (,was DENKSCH warum“, Z. 13),

- Nina markiert ihre Antwort explizit als Begründung (,wil“, Z. 14); und

- Patricks präsentiert seine Zurückweisung von Ninas Begründung als Folgerung („dann“, Z. 15), die sich zwangsläufig („müsst“) aus dem von Nina Gesagten ableitet, die aber im Widerspruch zur Realität steht (Konjunktiv II).

Schon in den ersten Zeilen des Transkripts wird deutlich, dass es in „Bartgeier“ um die Erarbeitung zoologischen Wissens geht. (Damit meine ich nicht ausschließlich komplexe fachliche Sachverhalte, die für Laien unverständlich sind, sondern jede Art von Wissen, das seine Relevanz aus seinem Bezug zur Wissenschaft der Zoologie bezieht.) Patrick interpretiert Ninas unspezifische Frage nach einem Objekt in der Vitrine (,un dÄ da OBbe?“, Z. 1) auf eine ganz spezifische Weise, nämlich als Aufforderung, den Namen der zoologischen Gattung anzugeben, die durch das betreffende Exponat repräsentiert wird. Sowohl in seiner klärenden Nachfrage (,dä Vogel?“, Z. 2) als auch in seiner anschließenden Antwort („das_sch_n BARTgEIer.“, Z. 3) verwendet er (alltägliche) zoologische Kategorien, um auf das fragliche Exponat zu referieren. Auch wenn diese Referenzformen 
auf den ersten Blick nicht besonders auffällig sind, ${ }^{19}$ zeigen sie uns doch, unter welchem Aspekt Patrick das Objekt in der Vitrine betrachtet: eben nicht unter einem Aspekt der Kaufbarkeit (wie beim Besuch eines Supermarkts, vgl. Stefani 2013 oder beim Durchstöbern des Museumsshops), und nicht unter Aspekten wie seinem praktischen Nutzen, seiner Ästhetik usw., sondern unter dem Aspekt seiner Verortung innerhalb einer (mehr oder weniger präzisen) zoologischen Taxonomie.

Nicht nur in der ,zeitlosen' Gesamtschau ist Wissen für das hier untersuchte Gespräch relevant. Wissen ist auch der entscheidende Motor der sequenziellen Organisation des Gesprächs. Denn dieses gewinnt seine Dynamik aus der Signalisierung von Wissensgefällen und deren sukzessiver Einebnung (vgl. Heritages Metapher der „epistemic engine“, Heritage 2012). Auch dieser Gesichtspunkt lässt sich in dem Ausschnitt „Bartgeier“ beobachten, etwa in den Zeilen 7 bis 26.

- Zunächst identifizieren Patrick und Nina ein Wissensgefälle. Patrick fragt mit „un WEISCH_d waRUM.“ (Z. 7) nach den Gründen für den Namen des Bartgeiers, und Nina gesteht: „das weiss i NÜD.“ (Z. 14).

- Dieses Wissensgefälle wird zum Motor des weiteren Gesprächsverlaufs. Denn in der Folge arbeiten der Vater und Nina gemeinsam daran, dieses Wissensgefälle einzuebnen.

- Die Beschäftigung mit dem Namen des Geiers wird so lange fortgesetzt, bis ein geteilter Wissensstand erreicht ist; hier dadurch, dass Patrick die Lösung des ,Namensrätsels“ verkündet und so für die Herstellung eines geteilten Wissens sorgt: „und darum heisst er BARTgeier.“ (Z. 26, s.a. Clark 1996: 222 zum ,principle of closure“)

Auch im nächsten - sehr kurzen - Gesprächsschritt ist die Herstellung von geteiltem Wissen für die Beendigung des Gesprächsschritts verantwortlich. Die Sequenz beginnt mit Ninas Behauptung, der Bartgeier fange Hasen (Z. 27). Dadurch dass Nina die von Patrick begonnene Äußerung „und DÄ:;“, mit der Aussage „tUEt (-) tuet_HÄSli fange.“ (Z. 27) interaktiv vervollständigt, erhebt sie den Anspruch zu wissen, welche Aussage ihr Vater zu dem Bartgeier machen wollte. Dieser Anspruch wird von Patrick ratifiziert. Er wiederholt Ninas Aussage und fügt ein lang gezogenes „’JA:,“ hinzu (Z. 28f.). Das Hasen-Fangen erhält so

19 Das ist die Leistung des semiotischen Arrangements im Ausstellungsraum, das wir in 3.6 rekonstruiert haben. Es lässt uns die zoologischen Kategorien gewissermaßen als die ,natürliche‘ Art und Weise erscheinen, sich auf das Objekt in der Vitrine zu beziehen. Die Beobachtung, dass diese Art der Kategorisierung des Exponats belegt, dass für die Gesprächspartner zoologisches Wissen relevant ist, wird durch diesen Eindruck von ,Natürlichkeit" aber nicht entwertet. 
den Status eines geteilten Wissens, also den Status einer Überzeugung, auf deren Gültigkeit sich beide Gesprächspartner festgelegt haben. Und wieder folgt auf das Festhalten der Geteiltheit eines Wissenselements ein neuer thematischer Schritt im Gespräch.

Der Ausschnitt „Bartgeier“ ist für die Gespräche meines Korpus in mehrfacher Hinsicht repräsentativ. Zum einen, was den wichtigen Stellenwert des Wissens und seiner lokalen Konstruktion in der Interaktion vor der Vitrine betrifft. Zum anderen im Hinblick auf seine sequenzielle Struktur: Das Signalisieren eines Wissensgefälles - sei es zwischen den Besuchern oder zwischen Besuchern und den Museumstexten und -exponaten - erweist sich im gesamten Korpus als ein prominenter ,Motor‘ der Interaktionsdynamik und das Herstellen von geteiltem Fachwissen als der zentrale Fluchtpunkt, auf den die Interaktion vor den Vitrinen im Ausstellungsraum hinausläuft. In diesem Sinn erscheint es mir gerechtfertigt, die Interaktionen vor der Vitrine hier vor allem als Wissenskommunikation zu verstehen und zu untersuchen (s. Kastberg 2007, Kesselheim 2010a). Wenn ich die Museumsbesuche hier als Wissenskommunikation untersuche, dann ist damit nicht gemeint, dass die gemeinsame Aktivität der Besucher ausschließlich im Herstellen geteilten Wissens zum ausgestellten Thema bestünde. Damit unterschlüge man die große Bedeutung, die dem Betrachten der Exponate für die soziale Praxis des gemeinsamen Museumsbesuchs zukommt. Das zeigt sich nicht nur in dem großen Aufwand, mit dem die Besucher dafür sorgen, dass sie das Betrachten der Exponate als gemeinsame Aktivität hervorbringen (s.o. 5.3), es zeigt sich auch in der Wissenskonstruktion selbst, die - wie ich gleich zeigen werde - immer wieder auf das Bezug nimmt, was im Ausstellungsraum zu sehen ist und von der Beobachtung spezifischer Merkmale an den Exponaten zur Konstruktion typischer Merkmale fortschreitet.

Schließlich ist festzuhalten, dass die Herstellung von geteiltem Wissen in den von mir dokumentierten Museumsbesuchen interaktiv erfolgt. Die Wissenskonstitution ist nicht einem einzelnen Interaktionspartner zuzurechnen, sondern immer die gemeinsame Leistung aller Beteiligten als ,Interaktionsteam‘. Auf den ersten Blick scheint das auf den Ausschnitt „Bartgeier“ nicht zuzutreffen. Denn zu Beginn des Transkripts positioniert sich Patrick seiner Tochter gegenüber als jemand, der über ein Wissen verfügt, das diese sich erst im Verlauf des Gesprächs erarbeiten soll. Doch die Behauptung, die Wissenskonstruktion erfolge hier interaktiv, impliziert nicht, dass alle Beteiligten mit dem gleichen Vorwissen und den gleichen Rollen an der Konstruktion des gemeinsamen Wissens beteiligt sein müssen. Gemeint ist vielmehr, dass die Interaktionsbeteiligten mit ihren Beiträgen zur Wissenskonstruktion jeweils ,online“ auf die Beiträge des anderen reagieren, dass sie also ihre eigenen Beiträge zur Konstruktion geteilten Wissens an den Schritt für Schritt entstehenden Kontext 
des Gesprächs anpassen. So tritt Patrick hier zwar als derjenige auf, der bei der Lösung des ,Namensrätsels‘ den führenden Part übernimmt, nämlich indem er Nina zur Entwicklung einer Hypothese anhält, ihren ersten Lösungsversuch kommentiert, ihr Gelegenheiten verschafft, über die eigene Anschauung auf die Lösung des Rätsels zu kommen, und indem er ihr schließlich die Lösung des Rätsels präsentiert. Doch reagieren seine Gesprächsbeiträge Schritt für Schritt auf die von Nina angezeigten kognitiven Vorgänge des Erschließens, Begründens, auf ihre Einnahme einer Betrachtungsposition usw. Ninas Verhalten wird also in dem von Heath/vom Lehn (2004) beschriebenen Sinn zur ,Ressource‘ für Patricks je nächsten Beitrag.

Die Behauptung, Wissenskonstruktion geschehe hier interaktiv, meint darüber hinaus, dass die Interaktionsbeteiligten gemeinsam bestimmen, was im weiteren Gespräch als Wissenslücke behandelt werden soll, die geschlossen werden soll (ein „learnable“ im Sinn von Reed/Szczepek-Reed 2014), was als geteiltes Wissen behandelt werden darf usw. Im Ausschnitt „Bartgeier“ erfolgt die Zuschreibung einer Wissenslücke an Nina durch beide Gesprächspartner: durch den Vater, der sich nach Ninas Wissen erkundigt (,un WEISCH_d wa waRUM“, Z. 7) und durch Nina, die erklärt „das weiss i NÜD.“ (Z. 14). Auch handeln Patrick und Nina zusammen aus, was jeweils als gemeinsames Wissen gelten soll. Der Bart am Geierschnabel als Motiv für die Namensgebung, der Hase als Beute, der Tod des Hasen, der schnelle Flug des Bartgeiers: all diese Wissenselemente werden bald von Patrick, bald von Nina ins Gespräch gebracht. Und der jeweils Zuhörende bestätigt das ins Gespräch gebrachte Wissenselement oder lässt die im Turn-Taking-System angelegte Gelegenheit verstreichen, den Gültigkeitsanspruch des soeben Behaupteten anzuzweifeln.

\subsubsection{Vom Sehen zum Wissen}

Im vorangegangenen Analyseabschnitt haben wir gesehen, dass die Besucher in ihrer Interaktion gemeinsam Wissen herstellen. Worin aber liegt nun die Museumsspezifik dieser gemeinsamen Wissensherstellung? Schauen wir uns dazu noch einmal den Ausschnitt „Bartgeier“ an.

\section{V8816 Bartgeier“ 1. Teil}

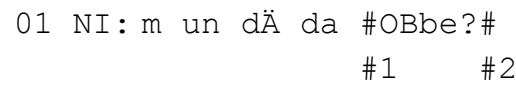



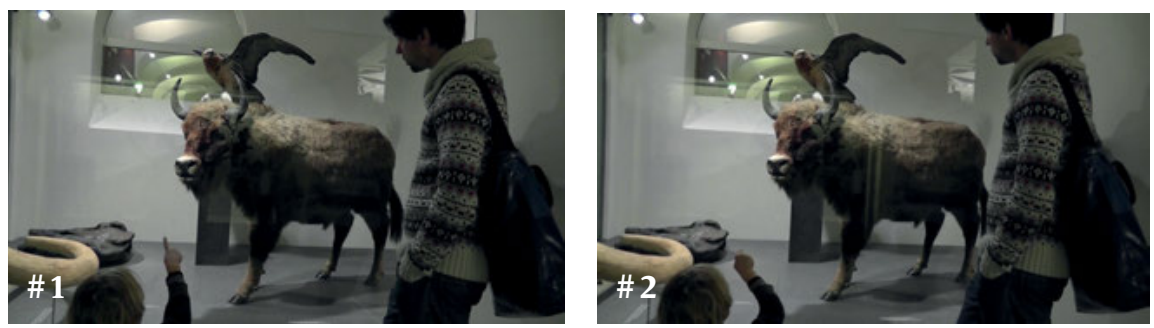

02 PA: dä VOgel? (1.0)\# (1.1) ah da stAht \#bim Vogel sch:t\# ah doch

\# 3

\section{\# 5}

03 hier drübe staht jetz !OH! das_sch_en BART\#gEIer. $(-)$

\# 6
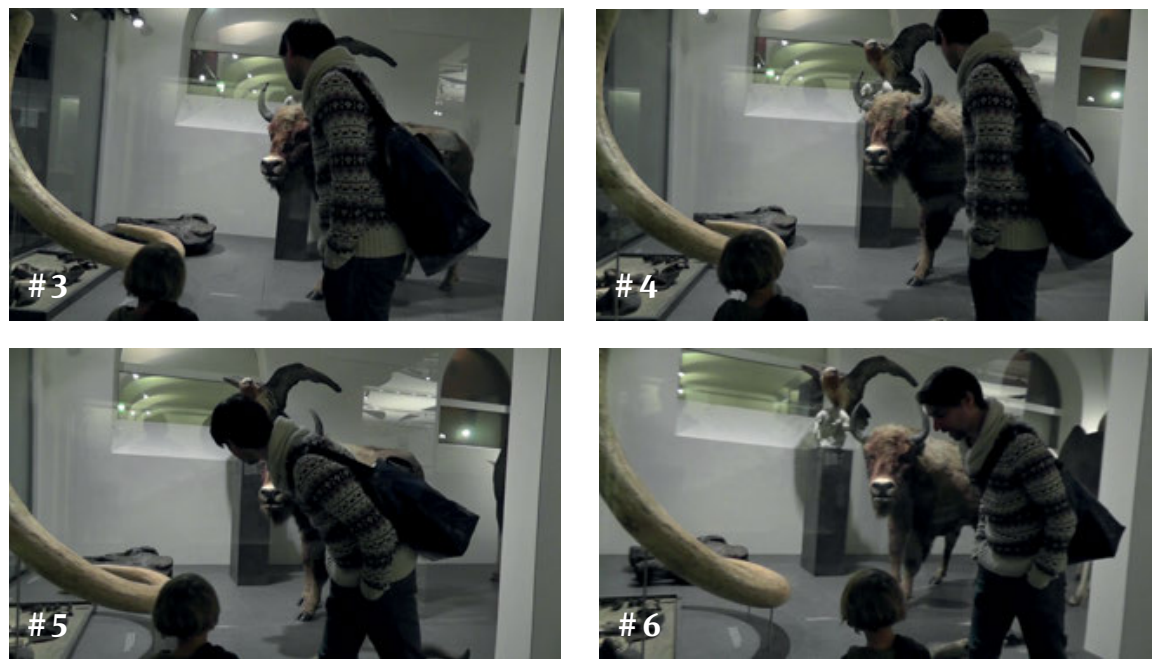

04 LUEG emal-

$05 \mathrm{NI}$ : woran GSEHSCH [du das? ]

$06 \mathrm{PA}: \quad[<<\mathrm{f}>\mathrm{HEI} .(-)]$

07 un WEISCH_d\# wa waRUM. $\# 7$

$08 \quad[($ dä_sch $)] \#$

09 NI: [<<ff $>$ ! $\ddot{A} !] \#$ dä hät es HÄSli gno:.\# \#8 

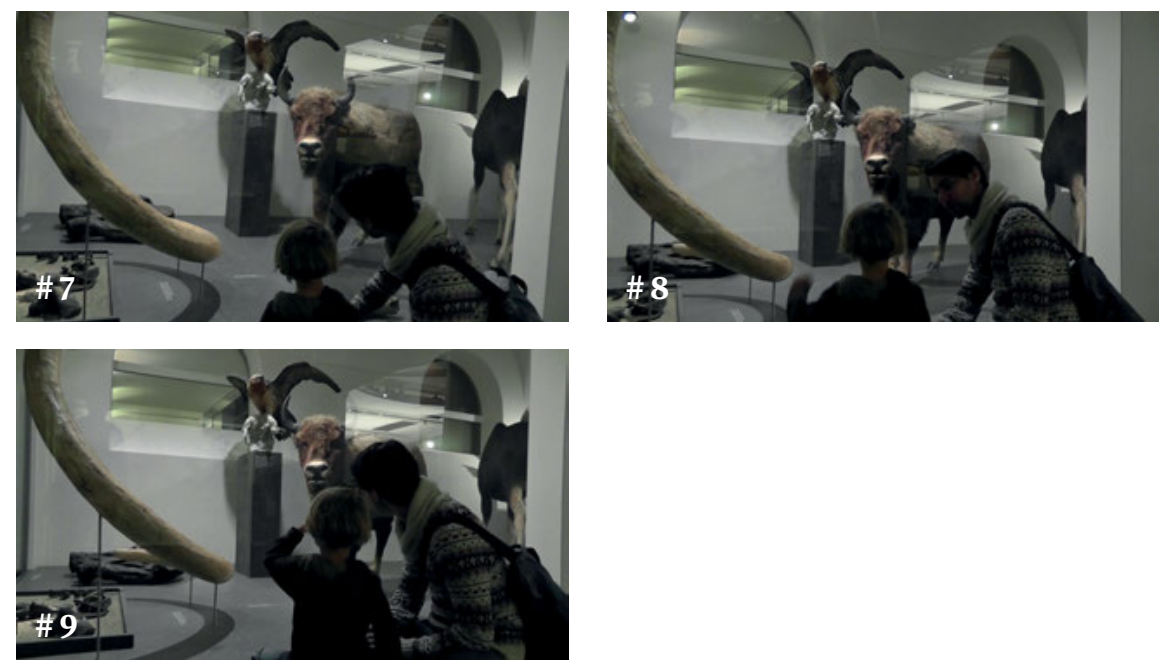

10

$(--)$

In diesem Ausschnitt nutzen Patrick und Nina den Ausstellungsraum bzw. das in ihm arrangierte multimodale Kommunikationsangebot als Ressource für ihre lokale Wissenskonstruktion. Dieser interaktive Bezug auf das in der räumlichen Umwelt Vorgefundene ist - um das wichtigste Ergebnis dieses Analyseabschnitts vorwegzunehmen - das Spezifikum der Wissenskonstruktion beim gemeinsamen Museumsbesuch. Wie zeigen sich also die Besucher in dieser kurzen Interaktionspassage, dass sie für ihre Wissenskonstruktion etwas in ihrer räumlichen Umwelt nutzen?

Erstens geschieht das hier durch das Mittel der Deixis. Mit Hilfe des Deiktikums das setzt Patrick die zoologische Kategorie Bartgeier und den gemeinsam etablierten Betrachtungsraum in Verbindung:: „das_sch_en BARTgEIer.“ (Z. 3, vgl. den auffälligen Blick ,um das Wisent herum‘: \#5). Diese Verbindung von Exponat und allgemeiner Kategorie, die in meinem Material äußerst häufig $\mathrm{zu}$ beobachten ist, signalisiert: Nicht die zoologische Kategorie an sich ist das Thema, sondern ihre Anwendung auf das in der Vitrine gemeinsam betrachtete Objekt, das Bartgeierpräparat.

Die zweite Verbindung von Wissenskonstruktion und räumlicher Umwelt geschieht über das Sichtbar- und Hörbarmachen der Lektüre von Texten im Ausstellungsraum; hier: einer Objektkennung unterhalb des Geierexponats.

- Patrick blickt auffällig in der Vitrine umher und führt Bewegungen mit dem Kopf aus, die nahe legen, dass er etwas sucht (\#3, \#4, \#5). Durch ,ah da stAht“ (Z. 2) zeigt er an, dass es sich bei dem Gesuchten um eine schriftliche Information handelt. 
- Durch „hier drübe staht jetz“(Z. 3) macht er explizit, dass er diese Information aus der Lektüre des Texts in der Vitrine bezieht (also nicht aus seinem Vorwissen) und verleiht dieser Information so den Status eines von der Autorität des Museums beglaubigten zoologischen Wissens. Durch das emphatische, die syntaktische Konstruktion seiner Äußerung unterbrechende „!OH!“ (Z. 3) hebt er den Neuigkeitswert dieser schriftlichen Information zusätzlich hervor.

Schließlich wird die Nutzung der räumlichen Umwelt durch die beiden Besucher auch offenbar, wenn sie sich die Relevanz des Sehens der Museumsdinge signalisieren. Explizit geschieht dies in Ninas Nachfrage zu Beginn des Ausschnitts: „woran GSEHSCH du das?“ (Z. 5). Offensichtlich reicht Nina die Autorität des Texts nicht aus, um Patricks Behauptung zu stützen, es handle sich bei dem Exponat um einen Bartgeier; sie fordert explizit eine Begründung, die sich auf die visuelle Evidenz in der Vitrine stützt. Implizit zeigt sich die Bedeutsamkeit des Sehens in der Museumsausstellung hier darin, dass Ninas Suche nach einem Benennungsmotiv für den Bartgeier gleichsam als ,Default‘ an dem ansetzt, was sie an dem Exponat sieht: den gefangenen Hasen in den Geierklauen („dä hät es HÄSli gnO:.““, Z. 9, \#9).

Die Besucher nehmen also in ihrer Interaktion auf Elemente in ihrem gemeinsamen Betrachtungsraum Bezug. Aber wie genau nutzen sie diese Elemente, um Wissen zu konstruieren? Eine grundlegende Vorgehensweise der Besucher in den von mir untersuchten Museen ist es, ausgehend von dem, was sie an dem ausgestellten individuellen Tierpräparat wahrnehmen, typische Merkmale der repräsentierten Gattung von Lebewesen abzuleiten. Ein Beispiel ist in dem folgenden kurzen Ausschnitt zu finden:

\section{V8816 „Bartgeier“ 2. Teil}
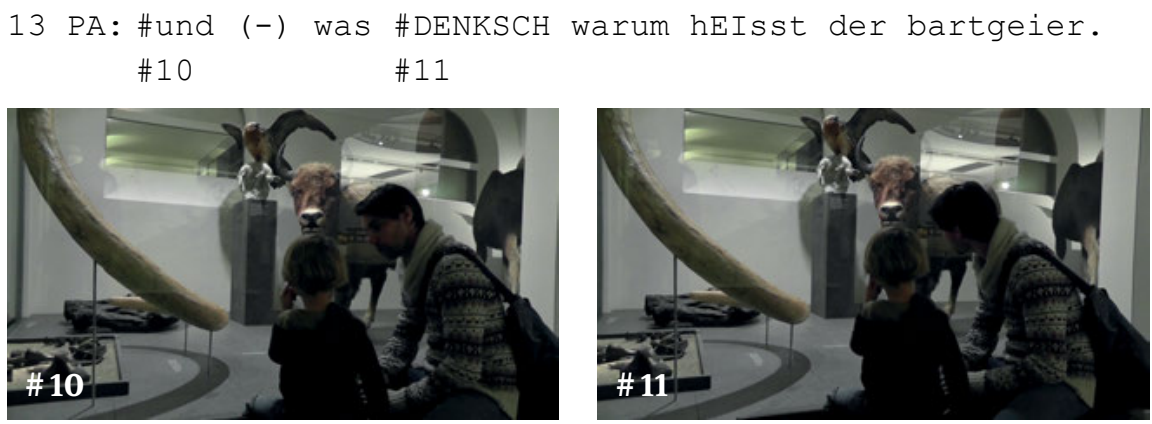

14 NI: das weiss i NÜD.\# (-) \#wil ${ }^{\circ} \mathrm{h}$ \#wil \#der es ${ }^{\circ} \mathrm{h}$ HÄSli nimmt ;

$$
\# 12 \quad \# 13 \quad \text { \#14 }
$$



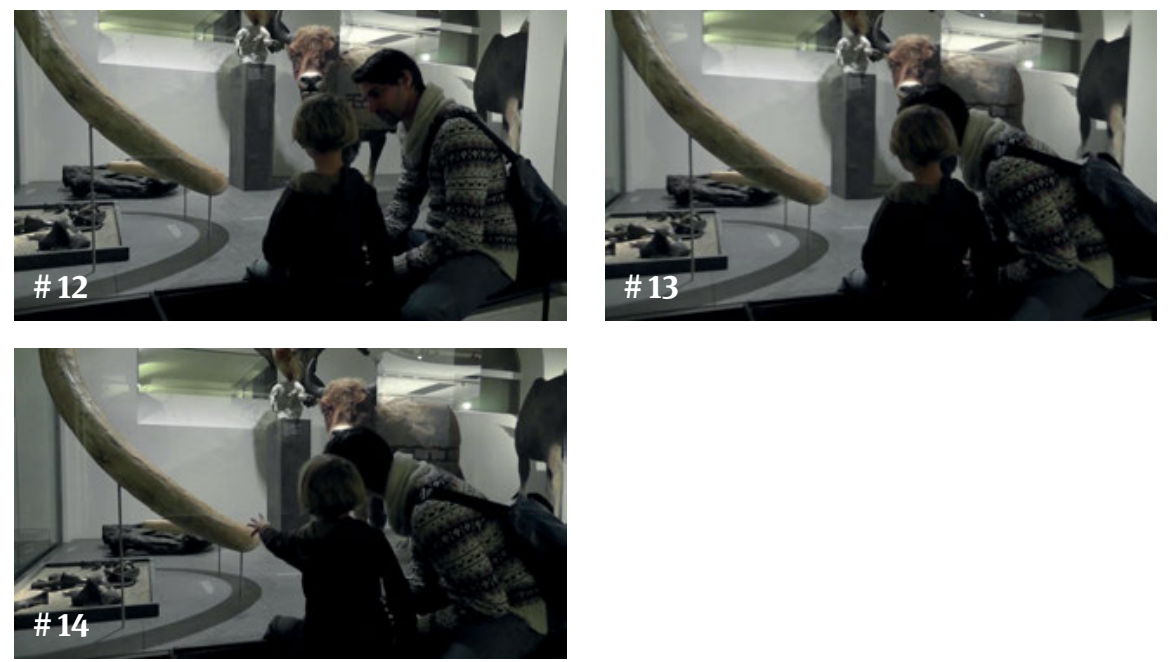

Vergleicht man Ninas Formulierung in Z. 14 mit ihrem ersten Antwortversuch (s.o., „Bartgeier“, Teil 1), dann fällt der Wechsel vom Perfekt zum Präsens ins Auge, also von „dä hät es HÄSli gnO:““ (Z. 9) zu „wil der es h HÄSli nimmt;“ (Z. 14).

- Während das Perfekt den Hasenfang als einmaliges Ereignis in der Vergangenheit beschreibt, dessen Folge in ihrem geteilten Wahrnehmungsraum zu sehen ist (,dä“ und die Zeigegeste in \#8 und \#9 im vorigen Transkriptausschnitt), drückt das Präsens die gleiche Aktivität nun als dauerhaftes Merkmal der Gattung Bartgeier aus. Nina spricht also nicht mehr von der Szene in der Vitrine, sondern von typischen Merkmalen der Gattung Bartgeier, die ja nicht im gemeinsamen Wahrnehmungsraum präsent ist. Und sie spricht sie als lebende Tiere an (indem sie davon spricht, dass sie Beute machen, wozu ja nur lebende Tiere in der Lage sind).

- In die gleiche Richtung geht Ninas Wechsel vom Singular (,es HÄSli“, Z. 9), der auf den im Moment des Sprechens sichtbaren Hasen referiert, zum Plural der Klasse von Hasen in einem späteren Moment des „Bartgeier“-Gesprächs (,[DÄ] tuet_HÄSli fange,“, der fängt die Hasen', Z. 27).

- Der Bezug auf die vom Exponat repräsentierte Klasse und ihre typischen Merkmale, geschieht nun nicht so, wie man in einer Erzählung oder einer Beschreibung über Abwesendes spricht. Vielmehr nimmt Nina im gleichen Moment, in dem sie der Klasse der Bartgeier typische Merkmale zuschreibt, auf etwas im gemeinsamen Betrachtungsraum Sichtbares gestisch und per Blick Bezug (\#12, \#14). 
Der hier vollzogene Übergang vom individuellen Exponat, das unter Verwendung von Teilen eines gestorbenen Tierindividuums erzeugt worden ist, zu einer Klasse von als lebendig behandelten Tieren sowie von während der Interaktion sichtbaren, individuellen Merkmalen hin zu typischen Merkmalen der Klasse ist uns aus dem Museum so vertraut, dass uns Ninas und Patricks Nutzung des Bartgeierexponats nicht als ungewöhnlich erscheint. Und doch haben wir es mit einer ganz spezifischen Nutzung von Elementen der räumlichen Umwelt zu tun, die auf ganz wenige Typen von Räumen begrenzt sein dürfte.

Wie wichtig das Erlernen dieser spezifischen Nutzung der Exponate für die Konstruktion von Wissen im Museum ist, zeigt beispielhaft der nächste Teil aus dem Ausschnitt „Bartgeier“. Hier arbeitet Patrick mit einigem Aufwand daran, sein Kind in den Stand zu versetzen, den Schritt vom in der Vitrine Sichtbaren hin zu allgemeinen Aussagen über die repräsentierte Tiergattung selbst zu vollziehen.

\section{V8816, „Bartgeier“ 3. Teil \\ 17 PA: m(u)sch e\#mal LUEge; =

$$
\text { \#15 }
$$ \\ 18 \\ \#=UNnen am schnAbel. \\ \#16}
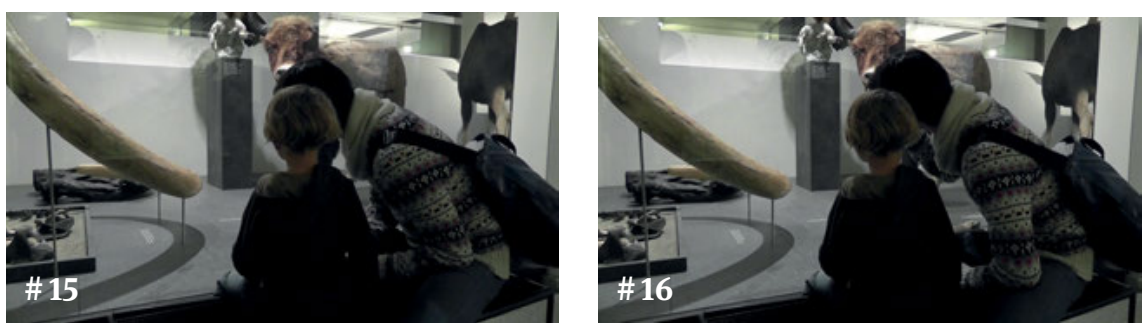

$19 \quad(1.0)$

20 GSEHSCH_des was er unnen am SCHNAbel hät.

21 NI: NEI,

$22(-)$

23 PA: vo de \#SIIt gsehsch (wemmer mal) uf dI siite \#gah, $(--)$ 

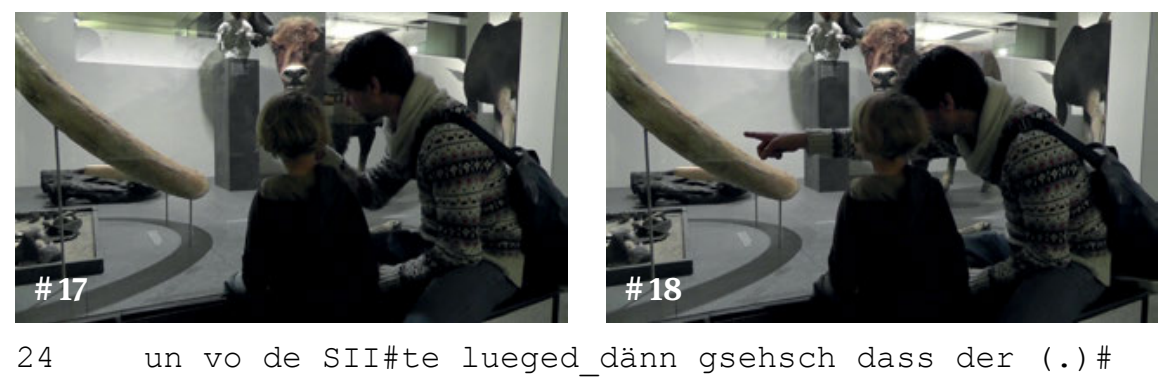

Unnen am

\#19

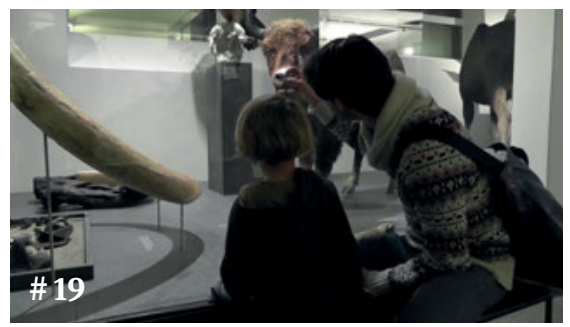

$\# 20$
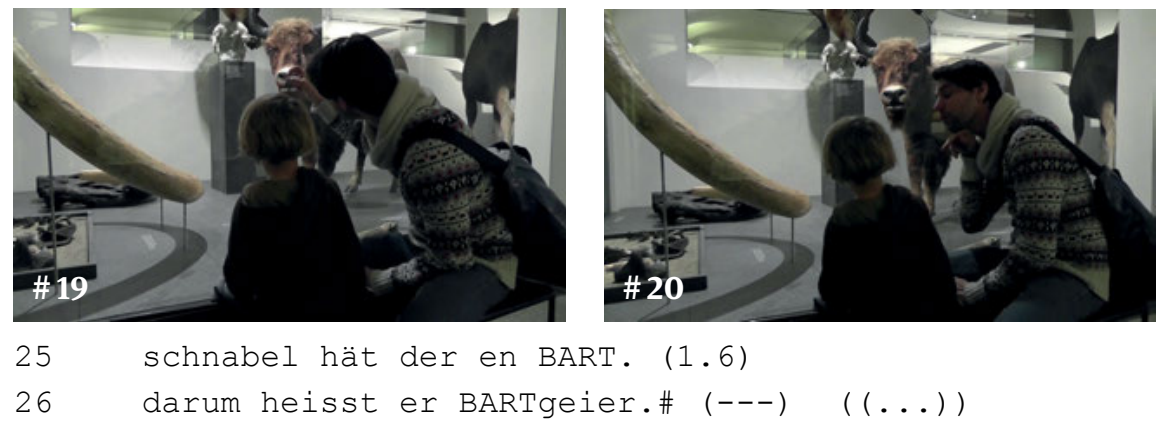

\#21

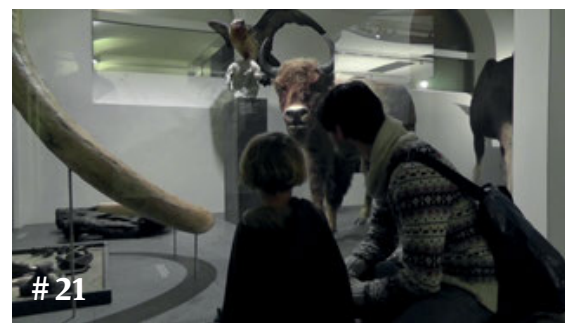

In diesem Fragment sehen wir: Es reicht Patrick nicht aus - ausgehend von den Informationen des Objekttexts beispielsweise - zu sagen: ,Der Bartgeier heißt so, weil er einen Bart hat. Hast du das gewusst?`. Stattdessen organisiert er in mehreren Schritten eine Perspektive für seine Tochter, von der aus sie das gesuchte Attribut des Geiers selbst sehen kann (s. dazu 5.3).

- Patrick fordert Nina ausdrücklich zum Schauen auf: durch „m(u)sch emal LUEge;“ (Z. 17) und „GSEHSCH_des was er unnen am SCHNAbel hät.“ (Z. 20). Dabei stellt er auffällig eine Sichtachse zum Exponat her (\#15, s. auch schon \#13 im 2. Transkriptteil. So unterstreicht er, dass sich das Namensrätsel mit Hilfe der Betrachtung des Exponats lösen lässt. 
- Daraufhin beschreibt Patrick aufwändig, von wo aus Nina etwas sehen könnte, was es ihr erlauben würde, das Rätsel selbst zu lösen (Z. 23 und 24), unterstützt von Gesten (\#17, \#18, \#19), die erst das Zielobjekt, dann den Betrachtungsstandort, dann wieder das Objekt identifizieren. Die auf \#19 dokumentierte Zeigegeste geht in eine ikonische Geste über, mit der Patrick an seinem Körper (s. dazu Hindmarsh/Heath 2003: 46) die Lösung des Namensrätsels präsentiert (\#20). Durch diese Reihe von Gesten macht Patrick seiner Tochter gleichsam den Lösungsweg sinnfällig, der von einer Betrachtung des Exponats von einem geeigneten Standpunkt aus (s.o. 5.3.4) zum Erwerb von Wissen über die repräsentierte Klasse von Tieren verläuft.

- Wenn Patrick schließlich schlussfolgert „darum heisst er BARTgeier.“ (Z. 26), leitet er wie selbstverständlich die Berechtigung des Gattungsnamens von der Beobachtung des einzelnen Exponats in der Vitrine ab, nutzt also das Exponat im gemeinsamen Betrachtungsraum zum Gewinnen allgemeiner, die gesamte Gattung betreffender Merkmale.

Wir können hier also sehen, wie Patrick daran arbeitet, seiner Tochter den Schluss von dem am Exponat Sichtbaren zu dem, was man über das Exponat wissen kann, zu ermöglichen. Dass diese spezielle Art der Nutzung des Exponats für Patrick einen ganz eigenen Wert hat, wird durch die Tatsache nahegelegt, dass Patrick andere, einfachere Möglichkeiten der Wissensvermittlung ungenutzt lässt und stattdessen aufwändig mit multimodalen Mitteln seine Tochter auf Betrachterperspektiven und sichtbare Eigenschaften am Exponat aufmerksam macht. Offensichtlich geht es Patrick hier nicht nur um die Vermittlung von Wissen zum Bartgeier, sondern auch um die Vermittlung der adäquaten Nutzung der Museumsdinge.

Eine gründliche Durchsicht des Gesamtkorpus bestätigt, dass dieses Übergehen von der Einzelbeobachtung am Exponat zur Zuschreibung allgemeiner Merkmale für die Wissenskonstruktion im Ausstellungsraum charakteristisch ist. Allerdings wird dieser Übergang selten als ein Gesprächsschritt realisiert, der scharfe Grenzen aufweist und der pro Wissenselement genau einmal vollzogen wird. Im folgenden Ausschnitt („Bartgeier“, 4. Teil), der an den soeben besprochenen unmittelbar anschließt, ist der Übergang vom Wahrzunehmenden zur Konstruktion allgemeiner Wissensbestände gleich in doppelter Hinsicht problematisch. $\mathrm{Zu}$ beobachten ist

- wie ein Interaktionsbeteiligter - hier ist es Nina - zwischen der Nutzung der Exponate als wahrnehmbarer Dinge im gemeinsamen Betrachtungsraum und der Nutzung als Zeichenkörper, die etwas repräsentieren, das nicht im gemeinsamen Wahrnehmungsraum präsent ist (hier: eine Gattung und ihre typischen Merkmale), hin- und herspringt; und 
- wie der gleiche Sachverhalt von dem einen Interaktionspartner als Beschreibung von etwas im geteilten Wahrnehmungsraum Beobachtbarem behandelt werden kann und von dem anderen als Beschreibung eines generellen Zusammenhangs, der durch die Objekte in der Vitrine als Zeichen repräsentiert wird.

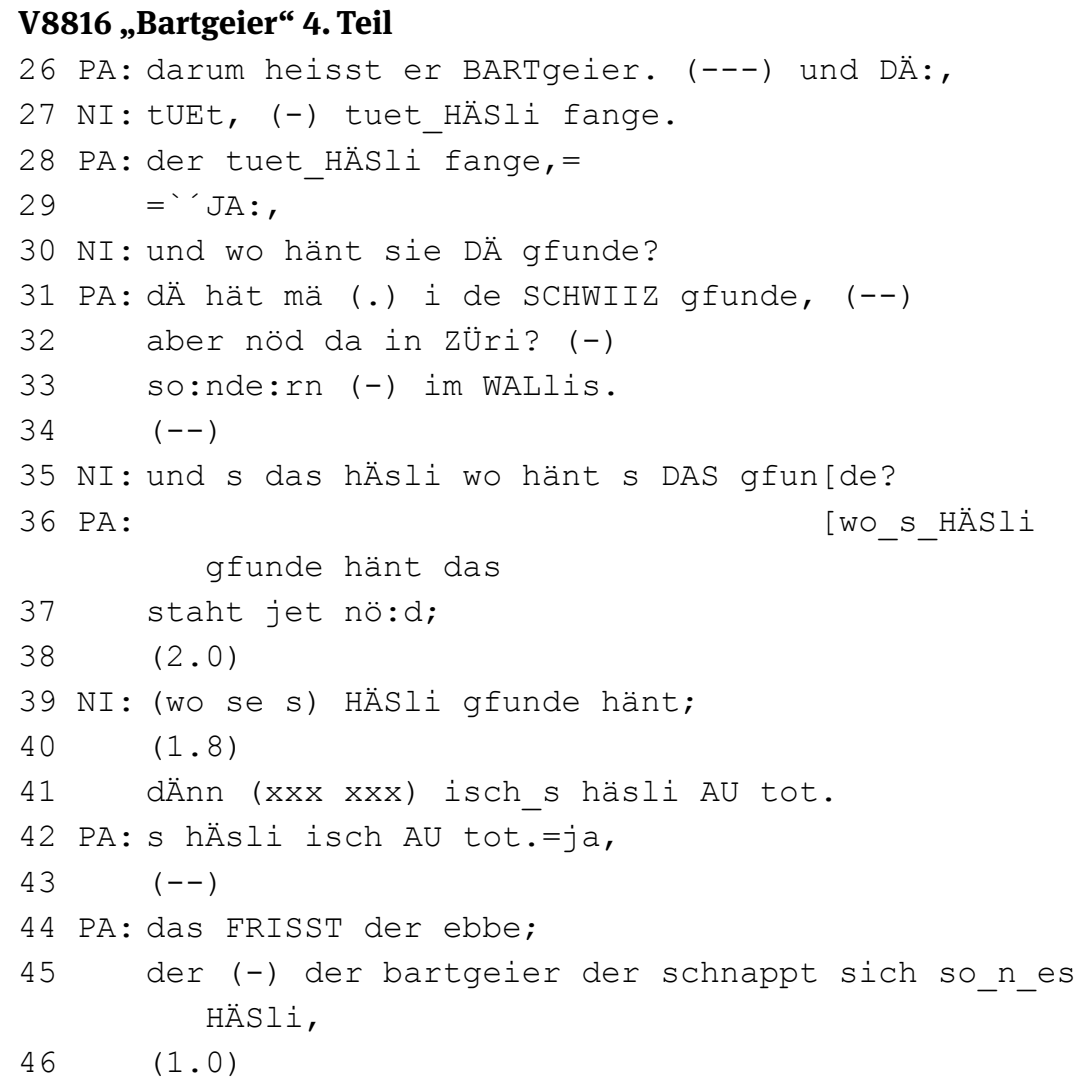

In ihrer ersten Äußerung schreibt Nina der Gattung Bartgeier das Fangen von Hasen als generelle Eigenschaft zu. Dafür spricht, dass sie von ,den Häslein` spricht (,tuet_HÄSli fange“, Z. 27, und nicht tuet es Häsli fange), obwohl in den Krallen des Bartgeierpräparats nur ein einziger Hase zu sehen ist. ${ }^{20}$ Dann springt sie aber zurück zum Sichtbaren: Die Frage „und wo hänt sie DÄ gfunde“ (Z. 30) kann man nur auf das individuelle Geier-Exemplar, nicht aber auf die Gattung

20 Ein weiteres Indiz ist, dass Nina eine Äußerung fortsetzt, in der Patrick über ein allgemeines Merkmal der Gattung Bartgeier gesprochen hat (die Motivation für seine Namensgebung). 
Bartgeier beziehen. Die gleiche Frage stellt sie mit Hinblick auf das Hasenpräparat, das sie in der Vitrine sieht (,und s das HÄSli wo hänt s DAS gfunde?“, Z. 35), um dann zu folgern („dÄnn“), dass es „AU tot“ ist (Z. 41). In beiden Fällen geht es ihr also um die Zuschreibung von individuellen Merkmalen an das Hasenpräparat, das Vater und Tochter in diesem Moment gemeinsam betrachten.

Der Vater dagegen bezieht die Frage danach, ob der Hase tot ist, auf die Gattung der Hasen als Beute der Gattung Bartgeier, arbeitet also anders als seine Tochter nicht daran, ein geteiltes Wissen zu dem unmittelbar in der Vitrine wahrnehmbaren Objekt zu etablieren. In seiner Äußerung ,das FRISST der ebbe; der (-) der bartgeier der schnappt sich so_n_es HÄSli,“ (Z. 45f.) spricht er vom JägerBeute-Verhältnis zweier Gattungen. An der Oberfläche macht sich dies neben dem generischen Präsens an der Referenz auf den Hasen als „so_n_es HÄSli“ bemerkbar (Z. 45). So ein referiert auf ein beliebiges Mitglied der Klasse der Hasen. Der in der Vitrine dargestellte Hasentod, wird von dem Vater als typisches Resultat der Nahrungsvorlieben ,des“ Bartgeiers beschrieben und mit Hilfe der Partikel „ebbe“ (Z. 44) als geteiltes Wissen ausgewiesen.

Obwohl sich beide Besucher den gleichen Wahrnehmungsraum teilen, arbeiten Vater und Tochter hier an unterschiedlichen Fragen: die Tochter verbleibt auf der Ebene des Sichtbaren, während der Vater an der Zuschreibung von Merkmalen arbeitet, die auf der Ebene der vom Sichtbaren repräsentierten Sachverhalte angesiedelt sind. Mit anderen Worten: die Besucher können die Exponate in ihrer Interaktion im Ausstellungsraum als Zeichen und als materielle Dinge betrachten und nutzen, wobei sich diese beiden Möglichkeiten nicht ausschließen müssen. Hier begegnet uns der Doppelcharakter der Museumsdinge wieder, der für die Rolle der Objekte im Museum zentral ist (Hahn 2016, Thiemeyer 2016).

\subsubsection{Einbezug von Vorwissen}

Bis hierher habe ich beschrieben, wie Besucher Wissen ausgehend von dem, was in der Ausstellung zu sehen und zu lesen ist, konstruieren: ausgehend von dem Bartgeierpräparat, an dem man den Bart erkennen kann, dem Hasenpräparat, das dessen Beute illustriert, und von dem Objekttext, der Informationen zu dem Geier bereitstellt. In dem nun folgenden Analyseabschnitt möchte ich komplexere Formen der Wissenskonstruktion betrachten: solche, bei denen die Interaktionsbeteiligten Vorwissen in die Wissenskonstruktion einbeziehen, und solche, in denen sie mehrere Exponate heranziehen, um sich einen zoologischen Sachverhalt zu erschließen. Hierzu werde ich Ausschnitte aus einer Videoaufnahme untersuchen, die den Besuch einer (standarddeutsch sprechenden) Mutter („Caroline“, FA) mit ihrem Sohn im Vorschulalter („Aaron“, AA) dokumentiert. Die beiden beschäftigen sich mit einer Vitrine zu eiszeitlichen Tieren. 


\section{V9759, „Elche“}

$01 \mathrm{CA}: \#<<$ behaucht $>$ !WO:W!.>

$\# 1$

$02 \quad(-)$

03 AA: GÄLL mami;

04 So \#hänt früher noch ${ }^{\circ} \mathrm{h}$ di_Elch usgseh[n.

$05 \mathrm{CA}$ :

[ja RICHtig;

\#2
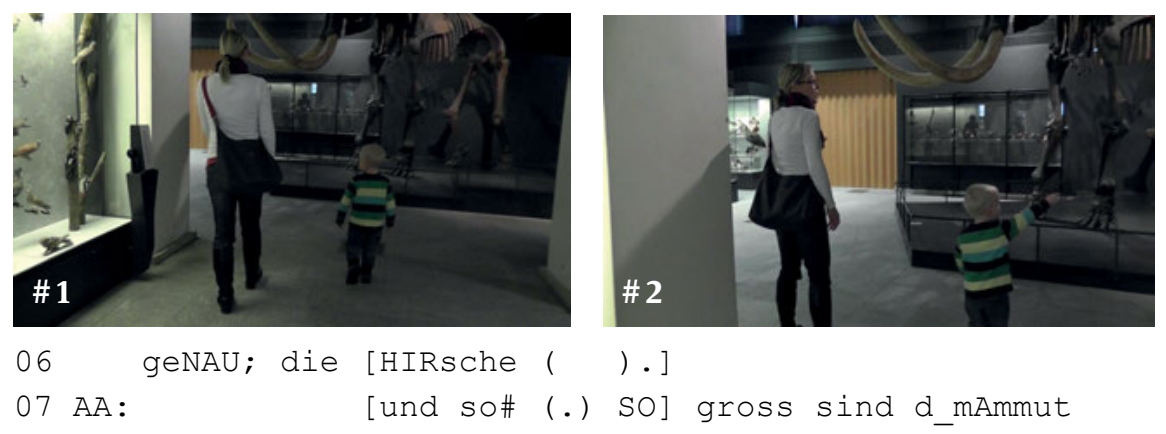

gsi.

\#3

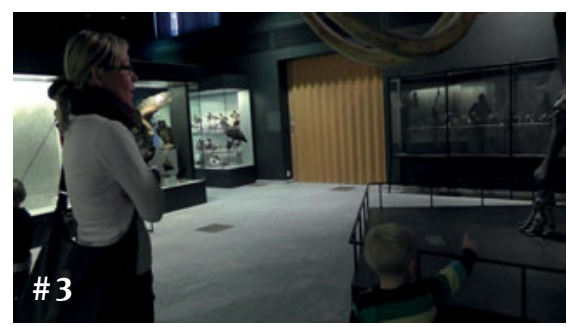

$08 \mathrm{CA}:$ geNAU.

09 aber \#das sind nUr noch die KNOchen vom mammut. =gell, \# \#4 \#5
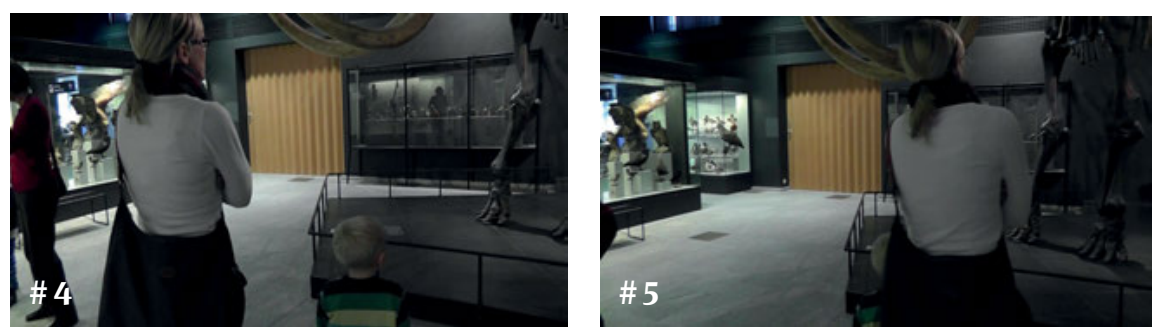

10 AA: GÄLL mami (-) gäll \#SO gross sind s wie d_elefAnte; 


\section{\# 6}

$11 \mathrm{CA}$ : NEIN elefanten sind kleiner.=

12 =mAmmuts sind VIEL grösser als die elefanten.

13 AA: \#sind das die GRÖSCHT_(-) _ti tIEr uf de welt; $\# 7$
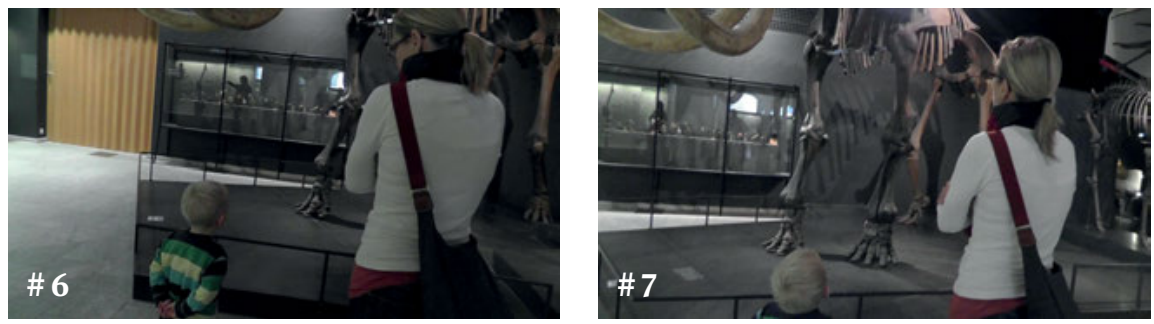

$14 \mathrm{CA}$ : die ALler allergrössten.

15 aber die leben jetzt NICHT mehr.=gell,

$16(-)$

17 AA: gits [noch gits gits (.) noch

$18 \mathrm{CA}$ : [die sind schon LANge lange AUsgestorben.]

19 AA: !NIE! GRÖSseri ${ }^{\circ} \mathrm{h}$ tiere als mammuts.

$20 \mathrm{CA}: \mathrm{NEI}$,

21 NEI,

22 (wArt mal / s_gab mal) (.) obWOHL die dinosaurier DIE warn

23 glaub ich noch grösser; (--)

24 aber \#DIE leben auch schon gAnz ganz lange nicht mehr;

\#8

$25(---)$

26 ganz ganz \#GANZ lang\# 9
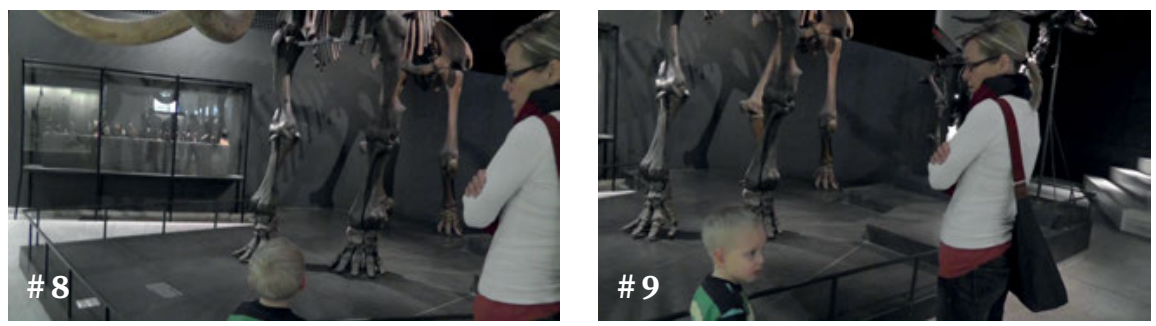

$27 \quad(--)$

28 GELL, \#

\#10 


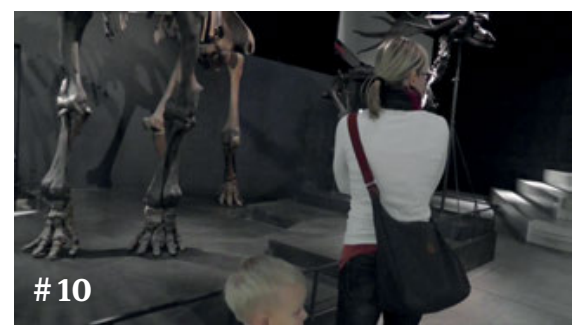

Die Erarbeitung gemeinsamen Wissens beginnt hier, gleich nachdem Caroline durch ein behauchtes „!WOW!“ ihre Überwältigung angesichts der großformatigen Exponate demonstriert (Z. 1, \#1) und so deren legitimen Status als museale Bewegungs- und Betrachtungsziele markiert hat (s.o. 5.2). Wie die beiden Besucher im letzten Beispiel behandelt der Junge Aaron die Objekte in seinem Gesichtsfeld als Zeichen, die auf etwas verweisen, das über das aktuell Wahrnehmbare hinaus geht. Aaron spricht von ,Elch' und Mammut im Plural („di_Elch“, Z. 4, und „d_mAmmut“, Z. 7), obwohl doch nur ein ,Elch“ (in Wirklichkeit ein Riesenhirsch) und ein Mammut zu sehen sind. Auch verwendet er ein Vergangenheitstempus („hänt [...] usgsehn“, „,sind [...] gsi“), obwohl die beiden Exponate aktuell im Ausstellungsraum zugegen sind. Und wie Nina und Patrick verbindet er die Aussagen zu den allgemeinen Eigenschaften der Gattung mit der aktuellen Wahrnehmungssituation. Er referiert nämlich gestisch (\#2) und mit dem modaldeiktischen so (eine „visuelle Evidenzkonstruktion“, Stukenbrock 2015: 419-424) auf sichtbare Merkmale im gemeinsamen Betrachtungsraum: „SO hänt früher noch ${ }^{\circ} \mathrm{h}$ di_Elch usgsehn. [...] und so (.) SO gross sind d_mAmmut gsi.“ (Z. 4, 17).

Anders als im letzten Beispiel bringen Caroline und Aaron aber auch Vorwissen ins Gespräch ein und verbinden dieses mit dem, was sie gemeinsam sehen. In Z. 10 vergleicht Aaron die Größe des Mammuts mit der der Gattung Elefanten (,gäll SO gross sind s wie d_elefAnte;“). Mit diesem Vergleich unterstellt er implizit, dass auch seine Mutter Caroline über dieses Vorwissen verfügt. Denn nur unter dieser Bedingung ist es hier sinnvoll, die Größe des Elefanten als Vergleichsmaßstab anzuführen.

Auch in den folgenden Äußerungen wird immer wieder Vorwissen zur Wissenskonstruktion herangezogen. Die beiden Besucher setzen das am Mammutexponat Beobachtete mit unterschiedlichen Elementen ihres Vorwissens in Verbindung und integrieren auf diese Weise neue Wissensbestände in ihr Vorwissen. So arbeiten Aaron und Caroline gemeinsam daran, die Größe der Mammuts in Bezug zu der Größe anderer Tierarten zu setzen, und zwar

- zur Größe aller lebender Tiere (,sind das die GRÖSCHT_(-) _ti tIEr uf de welt;“, Z. 13), 
- $\quad$ zur Größe aller jemals existierenden Tiere (,gits noch gits gits (.) noch !NIE! GRÖSseri ${ }^{\circ} \mathrm{h}$ tiere als mammuts.“, Z. 17, 19)

- $\quad$ und zur Größe der Dinosaurier („obWOHL die dinosaurier DIE warn glaub ich noch grÖsser;“; Z. 22f.).

Dabei begrenzt Caroline Schritt für Schritt die zeitliche Gültigkeit ihrer Aussagen auf die Zeit zwischen dem Aussterben der Dinosaurier (, aber DIE leben auch schon gAnz ganz lange nicht mehr; (---) GANZ ganz ganz lang-“, Z. 24, 26) und der Jetztzeit („die leben jetzt NICHT mehr“, „die sind [...] AUsgestorben“, Z. 15, 18). So wird im Verlauf der Interaktion von dem beobachteten Merkmal eines Exponats eine allgemeine Aussage zu einer Gattung abgeleitet und diese allgemeine Aussage sowie deren zeitliche Gültigkeit über wiederholte Vergleiche in das vorhandene Vorwissen (zur Größe anderer Tiere und zu deren Auftreten und Verschwinden im Laufe der Erdgeschichte) integriert.

Gerade diese Integration des Vorwissens, die hier so unauffällig geschieht, ist von der musealen Lernforschung als relevanter Faktor für das Lernen im Museum identifiziert worden (z.B. als Bestandteil des ,personal context' bei Falk/Storksdieck 2005). Hier können wir beispielhaft sehen, mit welchen konversationellen Verfahren genau die Besucher diesen Anschluss an das Vorwissen vornehmen und ihre Beobachtungen an das bereits Gewusste anschließen.

\subsubsection{Exponate als materielle Objekte und Zeichen}

Schon mehrfach haben wir gesehen, dass Besucher Exponate als Zeichen behandeln, die sie etwas erfahren lassen, das in der Wahrnehmungssituation nicht gegenwärtig ist: beispielsweise eine Klasse von ausgestorbenen Tieren deren Merkmale wie ihr Beuteverhalten (vgl. etwa Korff 1995: 24; s.a. Rheinberger 2013 $\mathrm{zu}$,epistemischen Dingen“). In den bisher analysierten Beispielen sind die Interaktionsbeteiligten routiniert mit diesem Doppelcharakter der Exponate umgegangen. Dieser unkomplizierte Umgang mit den Ausstellungsgegenständen verdeckt aber die Komplexität, die sich aus dem Charakter der Exponate als materieller Gegenstände ergibt.

Der Weg vom materiellen Zeichenkörper zum Bezeichneten ist längst nicht so unkompliziert, wie die bisher präsentierten Ausschnitte suggeriert haben mögen. Tatsächlich bringt das breite Spektrum des an den Objekten Wahrnehmbaren für die Besucher das Problem mit sich, dass sie entscheiden müssen, welche der zahlreichen sinnlich wahrnehmbaren Merkmale des Exponats sie als die für die Zeichenrelation relevanten verstehen wollen. Unsere Expertise in der Nutzung von Museumsausstellungen lässt uns in der Regel die Komplexität der Operatio- 
nen unterschätzen, die notwendig sind, um ein Verständnis zu entwickeln, was mit einem bestimmten Exponat ,gemeint‘ sein könnte.

Im Folgenden analysiere ich eine Interaktion, in der die semiotische Komplexität der Verbindung zwischen materiellen Objekten und ihrer Funktion als Zeichen zu einem Verständigungsproblem führt. Die ausführlichen Bemühungen, dieses Verständigungsproblem $\mathrm{zu}$ beseitigen, gestatten nicht nur einen guten Einblick in die Art und Weise, wie kompetente Museumsbesucher die kommunikative Funktion von Exponaten verstehen (und wie sie diese weitervermitteln). Sichtbar wird auch die Leistung von Sprache als einer hochspezialisierten Ausdrucksressource, ohne die die Vermittlung des komplexen semiotischen Funktionierens der Exponate nicht möglich wäre.

In der folgenden Interaktionsepisode zwischen dem Kleinkind Aaron und seiner Mutter Caroline werden wir sehen

- wie Aaron und seine Mutter Caroline mit der anspruchsvollen Aufgabe umgehen, mehrere Exponate aufeinander zu beziehen und sich so ausgehend von mehreren Exponaten ein gemeinsames Bezeichnetes erarbeiten; und

- wie Aaron und Caroline gemeinsam herausarbeiten, welche Merkmale dieser Exponate als stellvertretend für eine Klasse von Tieren verstanden werden dürfen (und welche nicht) und auf welches Bezeichnetes sie zu beziehen sind.

Am folgenden Ausschnitt („Knochen“) möchte ich dem ersten dieser beiden Punkte auf den Grund gehen. Nachdem Caroline und Aaron sich das große, freistehende Mammut angeschaut haben (s.o. „Elche“), wenden sie sich hier einer benachbarten Vitrine $\mathrm{zu}$, in der sich weitere Mammutknochen befinden (auf Abb. 5-28 rechts) sowie ein Abguss eines im Permafrost erhaltenen Mammutbabys (auf der Abb. links).

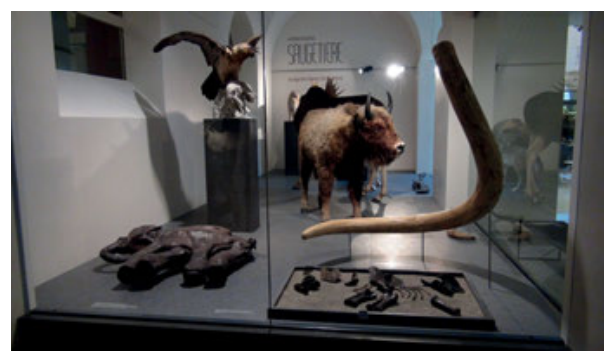

Abb. 5-28

\section{V9759 „Knochen“}

46 AA: KNOche sind \#das. \#1

$47 \mathrm{CA}$ : ' 'JA, 

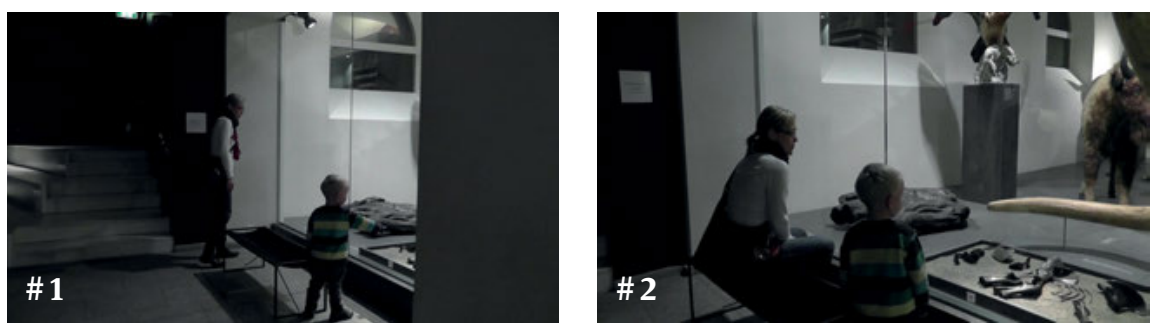

\section{$49(---)$}

50 AA: isch s mammutbaby (-) scho \#GSCHTORbe; \#3

51 CA: ja das is schon geSTORbe; =

52 =vielleicht war $\mathrm{s}$ krank, =

$53=$ GUCK ma \#da is es.

\# 4
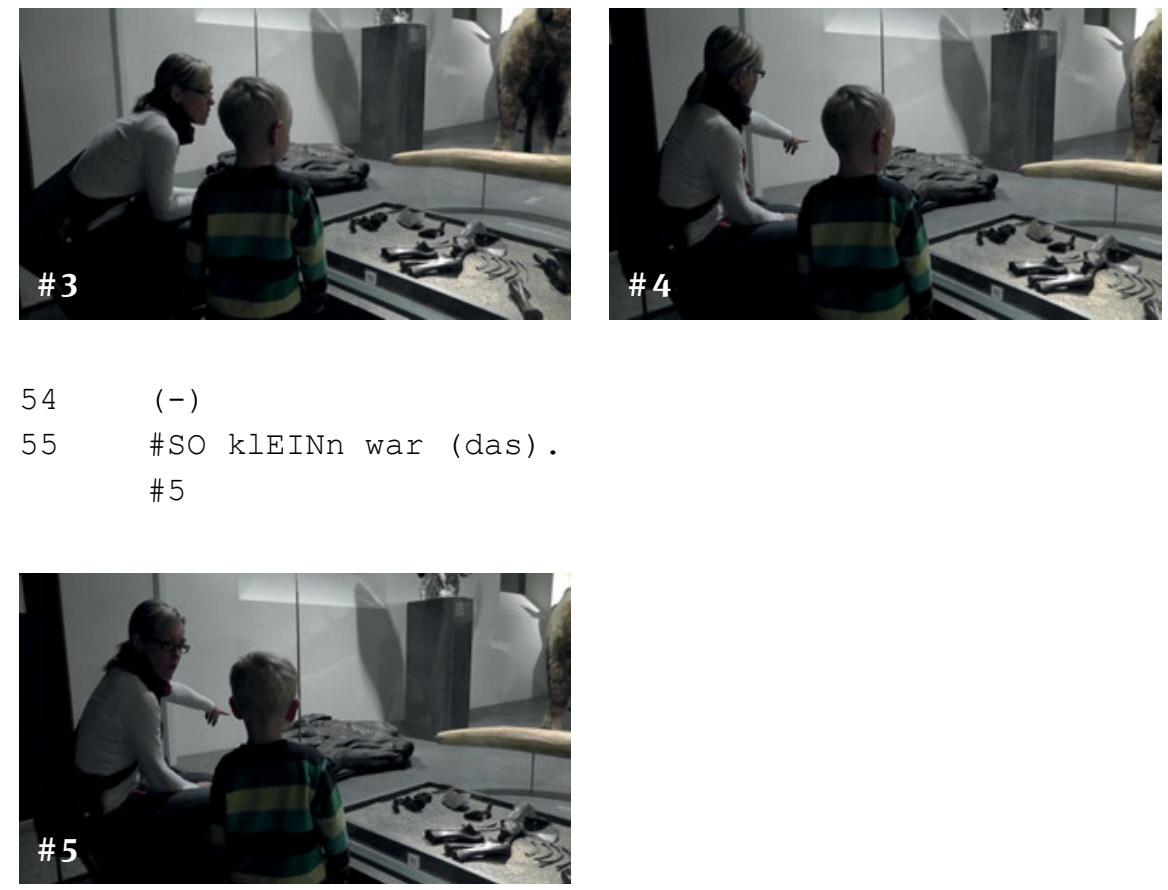
In diesem Gesprächsausschnitt bringen die Besucher zwei Exponate gleichsam ,zur Deckung‘, indem sie sie als zwei unterschiedliche Präsentationsformen konstruieren, die beide zusammen (nur) ein Lebewesen repräsentieren.

- Caroline und Aaron identifizieren gemeinsam ein am Vitrinenboden arrangiertes Exponat als Knochen vom Mammutbaby (Z. 46-48); vgl. die verbale und gestische Deixis von Aaron (,das“, Z. 46 und \#1) sowie die gemeinsame Blickorientierung von Aaron und Caroline auf \#2.

- $\mathrm{Zu}$ dem so identifizierten Objekt fragt Aaron „isch s mammutbaby (-) scho GSCHTORbe;“ (Z. 50, vgl. die Ausrichtung seines Kopfs auf das Knochenarrangement in \#3) und seine Mutter Caroline beginnt, über mögliche Gründe für den Tod des Mammutbabys zu spekulieren, wobei sie weiter auf die Knochen am Boden schaut (ebenfalls \#3).

- Durch eine Zeigegeste (\#4) und die Aufforderung „GUCK ma da is es“ (Z. 53) lenkt Caroline dann aber die Aufmerksamkeit ihres Sohns von den am Boden arrangierten Knochen weg und zu dem Plastikabguss des Mammutbabys weiter links in der Vitrine hin. Damit verlangt sie ihrem Sohn eine beträchtliche Interpretationsleistung ab. Denn er muss verstehen, dass das Mammutbaby gleichzeitig rechts in der Vitrine sein kann (,vielleicht war $s$ krank“, Z. 52) und links („da is es“, Z. 53; Zeigegeste \#4).

Die Mutter behandelt die Knochen rechts und den Abguss links als zwei Exponate im Betrachtungsraum, die zusammen der Konstruktion von unterschiedlichen Wissenselementen zu einer begrifflichen Einheit dienen: ,dem“ Mammutbaby. Das - und nur das - erlaubt es, die Aussage „SO klEIn war das“ mit Zeigegeste auf das linke Exponat (Z. 55, \#5) als Information auch zu dem rechten Exponat zu behandeln.

Dieses Beispiel zeigt: der Übergang von der Betrachtung der Exponate zu von ihnen repräsentierten Sachverhalten ist keinesfalls trivial. Wenn man die Ausstellung im Sinn der Ausstellungskommunikation nutzen möchte, muss man eine Expertise im Erschließen solcher Verbindungen von dem an den Exponaten Wahrnehmbaren zu allgemeinen zoologischen Sachverhalten erwerben. Denn der Schritt von dem, was man wahrnehmen kann, zu den ,dahinter stehenden' zoologischen Sachverhalten ist keineswegs fest in die Exponate ,eingeschrieben'. Wofür ein Exponat steht, muss jeweils von den Besuchern ausgehandelt werden. Diese Tatsache wird in der Regel durch unsere Routine im Umgang mit Museumsausstellungen verdeckt. Anders ist das in dem Gespräch von Caroline und Aaron, in dem die beiden Besucher nicht nur geteiltes Wissen über zoologische Themen herstellen, sondern auch ganz allgemein Wissen darüber, wie Exponate beim gemeinsamen Museumsbesuch genutzt werden können, um bestimmte, durch sie repräsen- 
tierte Sachverhalte zu erschließen. ${ }^{21}$ Dieses Thema durchzieht das Gespräch von Caroline und Aaron wie ein roter Faden. Dabei geht es um zwei Aspekte:

- Zum einen geht es den beiden Besuchern darum, was sie in der Vitrine und auf dem Podest hinter sich eigentlich sehen: Tierindividuen oder Repräsentanten, die für eine Klasse von Tieren stehen (und die über eine bestimmte Position in der zoologischen Taxonomie, über ihre Verknüpfung mit einer erdgeschichtlichen Epoche, ein bestimmtes Lebensalter usw. zu kategorisieren sind).

- Zum anderen geht es darum, welche der sichtbaren Merkmale zur Konstruktion von Wissen $\mathrm{zu}$ den repräsentierten Klassen von Tieren herangezogen werden dürfen, und welche nicht.

Diese beiden Aspekte sind in dem Gespräch von Caroline und Aaron eng miteinander verwoben. Deshalb möchte ich sie im Folgenden zusammen behandeln, um die übergeordnete Orientierung an der sequenziellen Organisation des Gesprächs beibehalten zu können. Um diesem Aspekt im Detail nachzugehen, werde ich nun eine Reihe aufeinander folgender Abschnitte aus dem Besuch von Caroline und Aaron untersuchen. Ich beginne mit zwei schon besprochenen Fragmenten aus dem letzten Ausschnitt („Knochen“).

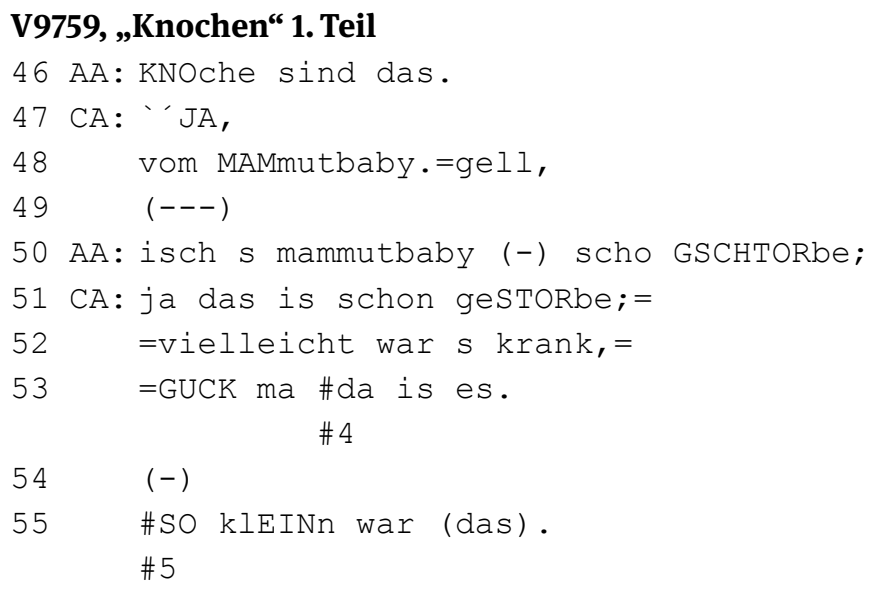

21 Wie Eltern ihren Kindern die Nutzung der Ausstellung und ihrer Exponate vermitteln können, untersucht beispielsweise Kelly (2007), eine inspirierende Analyse einer Eltern-Kinder-Interaktion vor einem Museumsexponat findet sich auch in Heath/vom Lehn (2001: 290f.). - Eine Orientierung an der Vermittlung institutionellen Wissens ist auch im Gespräch von Patrick und Nina zu beobachten. Siehe dazu beispielsweise den Ausschnitt „Bartgeier“. 

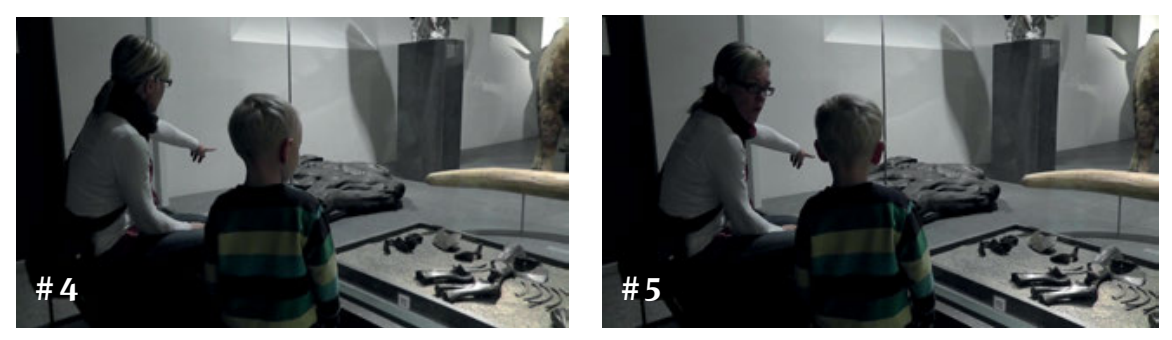

Ist hier von dem die Rede, was in der Vitrine wahrzunehmen ist, oder werden die Exponate der Vitrine als Zeichen behandelt?

- Beim Herantreten an die Vitrine identifiziert Aaron, wie schon gesagt, das Exponat auf dem Vitrinenboden zunächst als Knochen: „KNOche sind das.“ (Z. 46). Er bleibt mit seiner Beschreibung also nahe an dem, was in der Vitrine konkret zu sehen ist.

- Seine Mutter Caroline dagegen macht eine Aussage dazu, wofür das Wahrgenommene steht oder stehen kann: Indem sie die Knochen als Knochen „vom MAMmutbaby“ kategorisiert (Z. 48) spricht sie von ihnen als Zeugen für die Existenz eines bestimmten Tiers in einem bestimmten Lebensstadium.

- Aaron übernimmt in der Folge die Sichtweise der Mutter. In seiner anschließenden Äußerung spricht er nämlich nicht mehr von Knochen, sondern von dem Mammutbaby: „isch s mammutbaby (-) scho GSCHTORbe;“ (Z. 50). Es geht ihm um das konkrete Individuum, das in der Vitrine zu sehen ist, paraphrasiert: Sind die Knochen in der Vitrine ein Beleg für den Tod des individuellen Mammutbabys? ${ }^{22} \mathrm{Zu}$ dieser ,individuumsbezogenen' Lesart passt Carolines Reaktion, die ihre Vermutungen über die Todesursache mithilfe deiktischer Ausdrücke und Gesten im gemeinsamen Wahrnehmungsraum verankert (,da“, „es“, „das“, Z. 53, 55; \#4, \#5).

Die Frage ,Individuum‘ oder ,Repräsentant einer Klasse?‘ wird auch im 2. Teil des Ausschnitt „Knochen“ bearbeitet.

22 Denn das Verb sterben (in „GSCHTORbe“) kontrastiert mit dem Verb aussterben, das sich auf eine ganze Gattung bezieht und das Caroline in einer früheren Äußerung angesichts des großen Mammutskeletts verwendet hat. - In einem anderen Gespräch vor dieser Vitrine (V3310) wird der Unterschied von sterben und aussterben ebenfalls explizit thematisiert: ,jo das isch AU Usgstorbe. mhm, nach der geburt isch das geSTORbe hani das gfühl. ((...)) das is no nit emal richtig UFgstande. sIbe a nei da häsch_schteht da SIbe monat isch das alt. SIbe monat d (händ sie s) im im IIS gfunde im glEtscher inne isch das wie IIgfrore wie im iisfach. DOrum isches gfrore." 


\section{V9759 „Knochen“ 2. Teil}
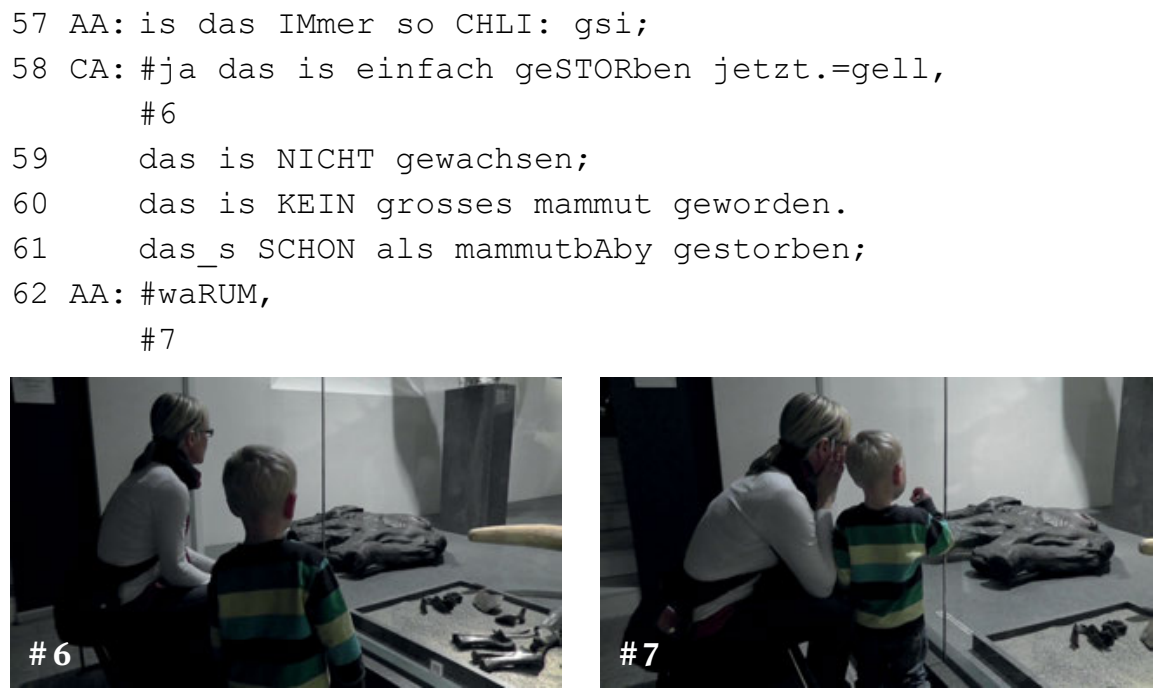

$63 \mathrm{CA}$ : ich WEISS es nicht; =

64 =vielleicht war $\mathrm{s}$ KRANK,

65 und dann is es geSTORben;

$66(---)$

67 So KLI isses gsi;

$68=$ WIE en kliner elefAnt; =gell?

Aaron wendet sich in diesem 2. Teil von „Knochen“ dem Problem zu, was ausgehend von dem visuellen Eindruck in der Vitrine gelernt werden kann. Konkret: Ist die sichtbare Größe des Mammut-Abgusses als Merkmal der Gattung aufzufassen oder als Merkmal eines Tierindividuums:

- Mit ,is das IMmer so CHLI: gsi;“ (Z. 57, \#6) fragt Aaron danach, ob die geringe Körpergröße zeitlich unbegrenzt gilt („IMmer“) und erfragt damit indirekt, ob das sichtbare Merkmal geringe Körpergröße als Merkmal der Gattung aufgefasst werden soll (Gattungsmerkmale sind ja prinzipiell zeitlich unbegrenzt gültig).

- Caroline ringt hörbar um eine Antwort auf diese Frage: „,ja das is einfach geSTORben jetzt.=gell, das is NICHT gewachsen; das is KEIN grosses mammut geworden. das_s SCHON als mammutbAby gestorben;“ (Z. 59-61, Kopfausrichtung bleibt wie in \#6). Zusammengenommen geht es ihr darum, die sichtbare Größe des Exponats als Resultat der individuellen Lebensgeschichte dieses individuellen Mammutexemplars und seines frühen Todes darzustellen (s. a. den Singular im abschließenden „so KLI isses gsi;“, Z. 67). 
und „PApi mammut“ (Z. 72, 73) ist in dieser Hinsicht uneindeutig. Sind Mutter und Vater des im Permafrost eingebetteten Mammutbabys gemeint, das sich in der Vitrine befindet, oder allgemein ein Muttertier oder Vatertier innerhalb einer Tierfamilie.

- In Carolines Antwort in den Zeilen 72 und 73 sind der Aspekt der Größe (als Merkmal eines ausgewachsenen Tiers) und der Aspekt der Rolle in einer Tierfamilie zunächst miteinander vermischt.

- Dann geht Caroline jedoch von „Mami“ und „PApi mammut“ zu „en erWACHSenes mammut en grOsses“ über (Z. 75).

- Aarons wiederholte Nachfragen führen also im Verlauf dieses Ausschnitts dazu, dass die anfängliche Vermischung aufgelöst wird. Schließlich wird nur der Wachstumsaspekt als gemeinsames Wissen festgehalten (,ja JA“, Z. 75).

Damit ist die Bearbeitung der Frage, welche Merkmale genau als repräsentativ für welche Klasse von Tieren stehen soll, beantwortet: Das Merkmal, um das es geht, ist die an der freistehenden Skelettrekonstruktion beobachtbare Größe; und diese darf als die Größe eines erwachsenes Exemplars der Gattung Mammut verstanden werden.

Der nächste Aspekt, den sich Aaron und Caroline an den Exponaten erarbeiten, betrifft die Frage, was die Knochen, die man in der Ausstellung zu sehen hat, mit den von ihnen repräsentierten Tieren zu tun haben.

In der Tat gibt das auf einen Metallrahmen montierte Mammutskelett ja einen Eindruck von dem Mammut, der weder dem entspricht, den irgendein Beobachter zu dessen Lebzeiten haben konnte - solange es lebte, war es ja von Fleisch, Muskeln, Haut und Fell bedeckt - noch dem, den man beim Auffinden des Mammuts hatte hier waren die Knochen nicht mehr im Verbund erhalten.

\section{V9759, „Knochen“ 4. Teil}

78 AA: isch das ! SO DÜNN! gsi;\#

\section{\# 12}

79 CA: ja weisste war ja noch HAUT und FELL über den knochen

84 AA: und wenn \#FELL darauf gsi is? 

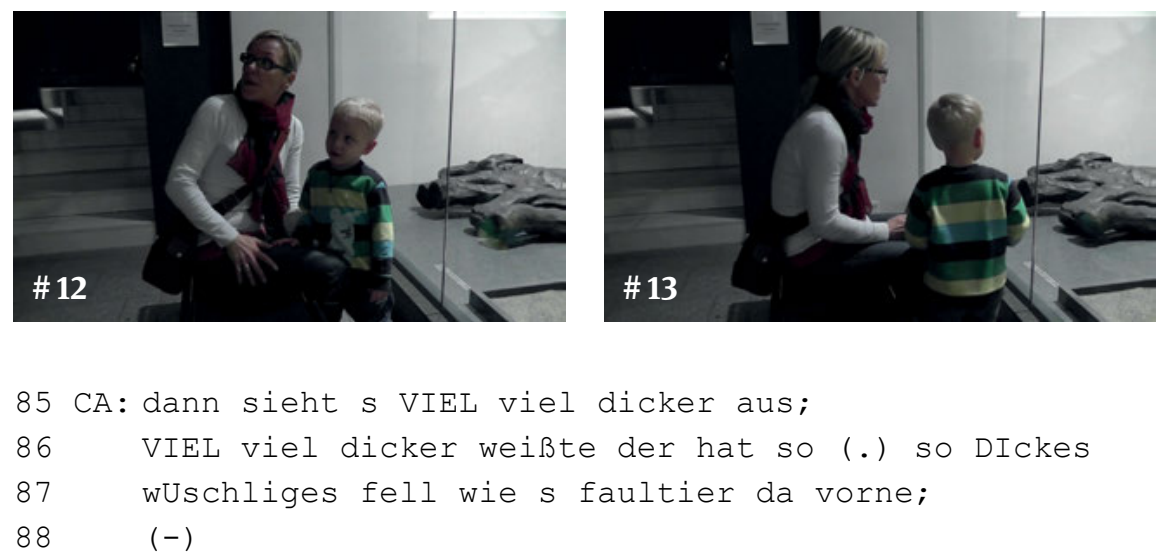

In diesem Ausschnitt geht es Aaron darum herauszufinden, welche der Wahrnehmungen am Exponat er nutzen kann, um etwas über das repräsentierte Lebewesen zu lernen. Konkret: Sind die Knochen das Mammut, oder sind sie es nicht?

- In ihrer Antwort konstruiert Caroline einen Gegensatz zwischen vergangenen Eigenschaften (Präteritum: „,war“, Z. 79f.) und der gegenwärtigen Wahrnehmung (,JETZT“, Wortformen von aussehen und sehen im Präsens, Z. 81f.).

- Gemeinsam verständigen sich die beiden nun darüber, dass es einen Unterschied zwischen dem am Exponat Sichtbaren und dem repräsentierten Tier gibt und dass für diesen Unterschied das Fehlen von Haut und Fell verantwortlich ist - vgl. das vom Sohn initiierte und von seiner Mutter vervollständigte Wenn-Dann-Format: „und wenn FELL darauf gsi is?“ - „dann sieht $\mathrm{s}$ VIEL viel dicker aus;“ (Z. 84, 85). ${ }^{23}$

Im nun folgenden Gesprächsabschnitt erarbeitet sich Aaron eine zunehmend generalisierte Regel, wie die Exponate im Zoologischen Museum in Beziehung zu den von ihnen repräsentierten Lebewesen stehen. Dazu zieht er ein Exponat heran, das beide zu Beginn ihres Besuchs betrachtet haben, die fellbedeckte Rekonstruktion eines Riesenfaultiers.

\title{
V9759 „Knochen“ 5. Teil
}

\author{
89 AA: ( (..)) (.) ${ }^{\circ} \mathrm{h}$ und wenn s FAULtier ${ }^{\circ} \mathrm{h}$ kei chnochen \\ 90 hät?
}

23 Schon durch Carolines „und da“ scheint mir eine kausale Relation angedeutet zu werden (,war ja noch HAUT und FELL über den knochen und $d a$ war s schon VIEL viel viel dicker;“ Z. 80). Explizit wird sie erst in der Wenn-Dann-Formel. 
So gelangt die Beschäftigung der beiden Besucher mit dem Status der Exponate zu einem (vorläufigen) Ende.

Am Ende des langen Aushandlungsprozesses, den wir hier über mehrere Transkriptausschnitte hinweg verfolgt haben, stehen sehr präzise Aussagen darüber, welche Schlüsse ausgehend von der Betrachtung der Mammutexponate legitim sind und welche nicht. Damit ist hier auch verhandelt worden, was an den Exponaten gelernt werden kann - ein zentraler Aspekt der Ausstellungskommunikation. Folgende Aspekte sind dabei thematisiert worden:

- Wie sind die Exponate aufzufassen - als Individuen oder als Vertreter einer Kategorie? Um welche Kategorie handelt es sich (eine Gattung wie Mammut, eines bestimmten Lebensstadiums wie Baby usw.)?

- Welche der beobachten Merkmale dürfen als für eine Gattung typisch begriffen werden?

- In welchem Verhältnis stehen die Exponate (Skelettrekonstruktionen, Einzelknochen, Präparate, Abgüsse usw.) zu den Objekten, die sie repräsentieren sollen. Ist der generalisierende Schluss von der beobachteten Größe auf die ,gemeinte“ Tiergattung erlaubt? Wie steht es mit dem Fell, der Farbe, seiner Position, dem wahrnehmbaren Kontext (vgl. den Bartgeier und ,sein` Häschen!)?

Wenn diese Frage im Ausschnitt „Knochen“ auch besonders ausführlich thematisiert worden sind, stellen sie sich doch prinzipiell bei jedem Museumsbesuch. Da es zwischen den Exponaten und dem, was sie repräsentieren, keine einfache Verbindung gibt, die sich direkt aus den Exponaten ergäbe, müssen die Besucher die Bedeutung der Exponate notwendig bei ihrer Rezeption konstruieren. ${ }^{24}$ Das gilt nicht nur, wenn Kinder die Ausstellung besuchen. Zwar sind Erwachsene, wenn sie erfahrene Museumsgänger sind, sicher insofern im Vorteil, als sie über ein differenzierteres Wissen dazu verfügen dürften, was ausgestopfte Tiere sind, was Skelett-Rekonstruktionen, was versteinerte Knochen usw. Doch bleibt auch für sie die Konstruktion der Exponatsbedeutung eine grundlegende Aufgabe, der die sie sich bei jedem Besuch stellen müssen.

In den vorangegangenen Analysen habe ich rekonstruiert, wie die Besucher sich ausgehend von dem, was sie im Ausstellungsraum wahrnehmen und lesen, (Fach-)Wissen erarbeiten. Wir konnten sehen, dass die Herstellung dieses Wissens interaktiv erfolgt und dass das Signalisieren von Wissensgefällen sowie

24 Dabei können sie nicht nur auf die sinnliche Wahrnehmung der Exponate zurückgreifen, sondern, wie wir in Kapitel 3 gesehen haben, auf eine große Vielfalt multimodaler Zeichen im Ausstellungsraum, die ihnen Hinweise geben, wie die Exponate aufzufassen sind (s. auch $\mathrm{u}$. Kapitel 6). 
das Ausgleichen dieser Gefälle für die sequenzielle Gestalt der Gespräche vor den Vitrinen wesentlich ist. Diese spezifische, die räumliche Umwelt kommunikativ nutzende Art der Wissenskonstruktion ist eine grundlegende gemeinsame Aktivität innerhalb der sozialen Praxis des gemeinsamen Museumsbesuchs. Aber haben wir hier wirklich Wissen untersucht? Die rein äußerlichen Beobachtungsdaten meines Korpus‘ erlauben schließlich keinen direkten Zugriff auf die Lernvorgänge im Innern der Museumsbesucher (s.o. 3.2.5 zur „Unbeobachtbarkeit von Wissen“). In der Tat ist Wissen hier konzeptualisiert worden als ein von den Interaktionsbeteiligten gemeinsam im Gespräch etablierter Status von Sachverhalten: Als Wissen gilt, was sich die Interaktionsbeteiligten gemeinsam als zutreffend (häufig auch: als allgemein bekannt und als allgemein gültig) signalisieren.

Was ist aber, wenn sich die im Gespräch erarbeiteten gemeinsamen Überzeugungen von dem unterscheiden, was die Wissenschaft herausgefunden hat? Wenn Caroline und Aaron beispielsweise überzeugt sind, dass sie einen Elch betrachten, während sie tatsächlich vor einem Riesenhirsch stehen? Der Abgleich des lokal konstruierten Wissens mit dem wissenschaftlichen Fachwissen scheint mir nur aus einer angewandten Perspektive nützlich zu sein. Dann also, wenn man wissen möchte, ob die Besucher das gelernt haben, was sich die Kuratoren von dem Arrangement in der Vitrine erhofft haben. Die Analysen dieses Kapitels dagegen haben die grundlegende ,Mechanik` der Wissenskonstruktion beim gemeinsamen Museumsbesuch zum Gegenstand. Sie erlauben es zu rekonstruieren, welche kommunikativen Verfahren die Besucher anwenden, wenn sie das Arrangement im Ausstellungsraum nutzen, um sich Fachwissen zu erschließen. Zur Musterhaftigkeit dieser Verfahren gehört unter Anderem,

- wie sie die Elemente der räumlichen Umwelt als ,Wissensdinge“ adressieren, also als bedeutungsvolle Objekte, aus deren Betrachtung sich etwas lernen lässt;

- wie sie sich gegenseitig aufzeigen, dass ihre Beobachtungen und Behauptungen sich auf wissenschaftliche Erkenntnisse, Aktivitäten oder Akteure beziehen; oder

- $\quad$ wie sie spezifische Instanzen zur Stützung ihres behaupteten Wissens heranziehen (beispielsweise Ausstellungstexte, eigene Beobachtungen oder Verweise auf wissenschaftliche Bücher oder Sendungen).

Ich bin überzeugt, dass die vorausgegangenen Analysen den Nachweis erbracht haben, dass es methodisch sinnvoll und inhaltlich ergiebig ist, die Videoaufnahmen authentischer Museumsbesuche zu nutzen, um die Konstruktion von Wissen als kommunikativen Prozess zu untersuchen. Zwar stimmt es, dass wir auch nach der Analyse nicht mit Sicherheit wissen können, was Patrick und Nina 
beim Nutzen der Bartgeiervitrine gelernt haben (s.o. „Bartgeier“). Und dennoch konnten wir eine Reihe substanzieller Beobachtungen machen, nämlich

- wie die Interaktionsbeteiligten einen Sachverhalt identifizieren, zu dem es ein Wissensgefälle gibt (sei es zwischen den einzelnen Mitgliedern der Besuchergruppe, sei es zwischen Besuchern und dem in der Ausstellung repräsentierten Fachwissen) und das heißt: wie sie definieren, was das „learnable“ für sie ist (Reed/Szczepek-Reed 2014),

- welche allgemeinen und museumsspezifischen Verfahren sie anwenden, um dieses Wissensgefälle einzuebnen (konkret: indem sie sich auf Wahrnehmungen an den Exponaten beziehen und Hypothesen entwickeln, welche dieser Wahrnehmungen welche zoologische Sachverhalte repräsentieren sollen; s. dazu besonders das Gespräch von Aaron und Caroline,

- wie sich die Interaktionspartner auf einen fachlichen Wissensbestand beziehen (etwa wenn Patrick die Exponate in zoologische Kategorien einordnet), oder

- wie sie gemeinsam Aussagen über die ,Welt‘ (im Sinne einer außersprachlichen Realität) erarbeiten, die von ihnen als geteilte Gewissheiten behandelt werden.

Kurz: Wenn wir auch nicht, in die Besucher schauen' konnten um zu erfahren, was die Besucher im Einzelfall lernen, so haben die Analysen doch einen detaillierten Einblick in einen zentralen Bestandteil der sozialen Praxis des gemeinsamen Museumsbesuchs ermöglicht. Sie rekonstruieren die kommunikativen Verfahren, mit denen die Besucher beim gemeinsamen Gang durch das Museum den Ausstellungsraum als „Lernort“ benutzen. Sie erlauben es, das beobachtete Verhalten der Besucher mit den Bedeutungspotenzialen im Ausstellungsraum in Beziehung $\mathrm{zu}$ setzen, die in Kapitel 3 rekonstruiert worden sind, und beschreiben, wie die Wissenskonstruktion der Besucher an diesen Potenzialen anknüpft (oder sich über sie hinwegsetzt).

Die Alternative $\mathrm{zu}$ diesem strikt oberflächenorientierten Verständnis von Wissen bestünde darin, die Interaktionsdaten als ,Fenster` $\mathrm{zu}$ den individuellen Lernprozessen zu verstehen (wie der Ansatz der „conversational elaboration“ innerhalb der musealen Lernforschung, s.o. 3.1). Dieser ,Kurzschluss` von Interaktionsdaten zu mentalen Vorgängen und Repräsentationen scheint mir gerade in Anbetracht der neueren, weiteren Auffassung von „learning outcomes“ problematisch zu sein (s. Rennie/Johnston 2007, Hooper-Greenhill 2008 oder Allen/ Peterman 2019), von denen viele sich sprachlich kaum oder nur schwer fassen lassen dürften. 


\subsubsection{Sind im Museum Wahrnehmungsraum und Handlungsraum deckungsgleich?}

Anders als in den vorausgehenden Analyseabschnitten, in denen ich untersucht habe, wie die Besucher einen gemeinsamen Bewegungs- und Betrachtungsraum etablieren (s.o. 5.2 und 5.3), habe ich mich im gegenwärtigen Abschnitt bisher noch nicht mit der Frage beschäftigt, wie im Zuge des Museumsbesuchs ein gemeinsamer Handlungsraum hervorgebracht wird und worin die Museumsspezifik seiner Hervorbringung liegt. Der Grund hierfür liegt in der essenziellen Rolle, die das Betrachten für die Praxis des gemeinsamen Museumsbesuchs spielt. Ohne das Betrachten der Exponate im gemeinsam hergestellten Wahrnehmungsraum wäre ein Gang durch die Ausstellungsräume kein Museumsbesuch. Wenn also das Betrachten für den Museumsbesuch derart grundlegend ist, sind dann nicht Wahrnehmungsraum und Handlungsraum einfach gleichzusetzen? Ist es nicht ein Charakteristikum der Kommunikation in der Ausstellung, dass Betrachtungs- und Handlungsraum in eins fallen?

Im Folgenden möchte ich zunächst Beobachtungen anführen, die für eine Gleichsetzung von Betrachtungs- und Handlungsraum sprechen, bevor ich dann Fälle untersuchen werde, die zeigen, dass es trotz der großen Bedeutung des Betrachtungsraums für die Interaktion im Ausstellungsraum sinnvoll ist, diese beiden Räume analytisch voneinander zu trennen.

Was also spricht dafür, die Etablierung des gemeinsamen Betrachtungsraums in der Vitrine gleichzeitig als Etablierung eines musealen Handlungsraums aufzufassen? Ein Argument hierfür ist, dass die Konstruktion von Wissen in der Regel genau in dem Moment beginnt, wenn die Besucher den gemeinsamen Betrachtungsraum in der Vitrine hervorgebracht haben. Auch bezieht sie sich mit wenigen Ausnahmen auf Objekte innerhalb dieses gemeinsamen Betrachtungsraums (die Exponate). Der Betrachtungsraum schafft also die Voraussetzungen für die gemeinsame, raumbasierte Wissenskonstruktion in der Ausstellung.

Das lässt sich im folgenden Ausschnitt beobachten (ein Ausschnitt aus dem schon analysierten Transkript „Elche“, s.o. 5.4.2.2, aber mit einigen Sekunden Vorlauf und zusätzlichen Standbildern). Der Ausschnitt dokumentiert, wie Caroline und Aaron ihre Beschäftigung mit ,Elch` und Mammut beginnen.

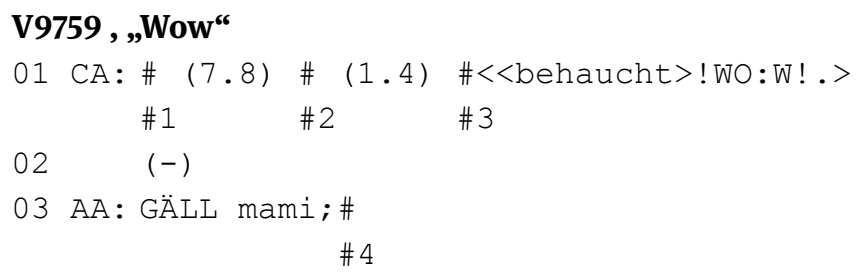



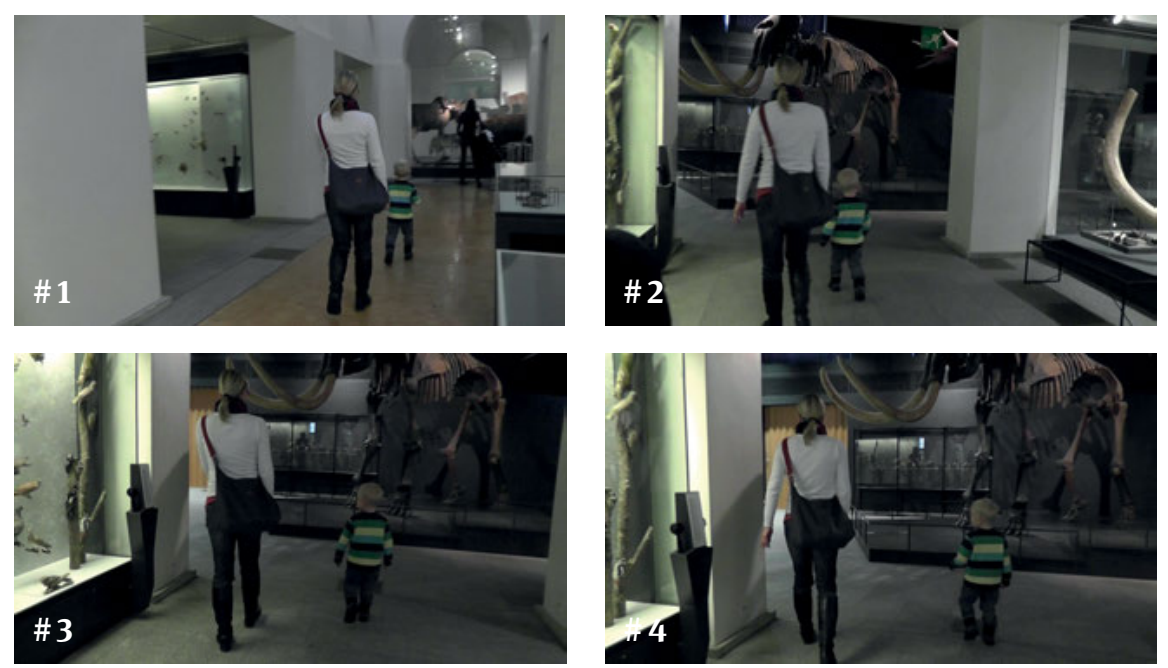

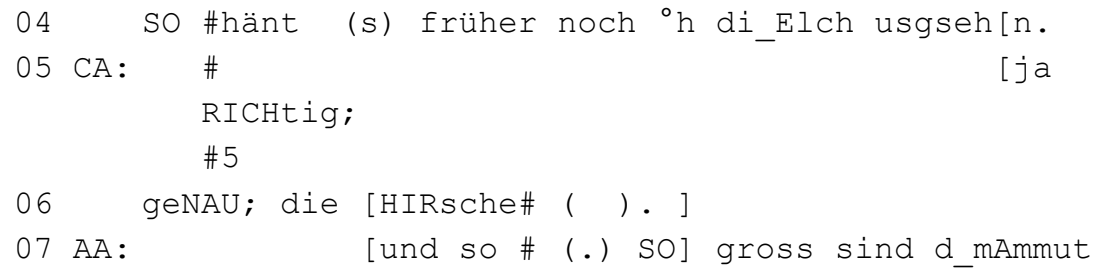
gsi.

\#6
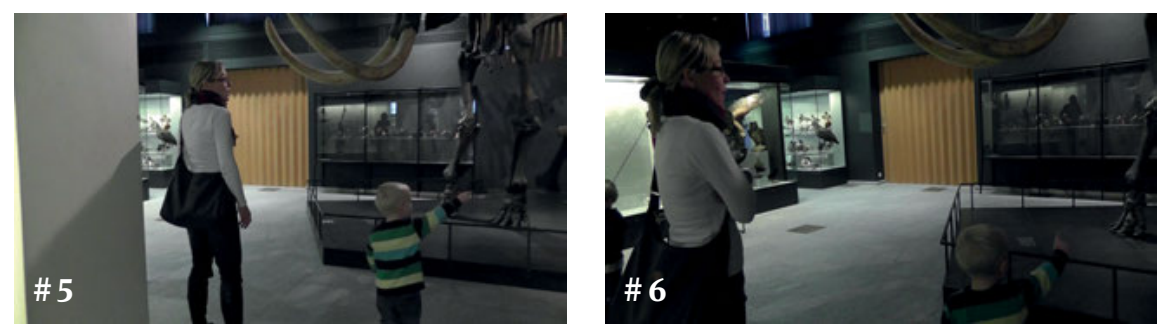

Hier möchte ich verfolgen, wie die beiden Besucher aus der Bewegung heraus zum Stillstand kommen und einen gemeinsamen Betrachtungsraum etablieren:

- Der Beschäftigung mit Riesenhirsch und Mammut im Ausschnitt „Wow“ geht eine längere Bewegungsphase durch den Ausstellungsraum voraus. 
Zunächst laufen Mutter und Sohn hintereinander (\#1). ${ }^{25}$ Doch als sie in die Nähe des Mammutskeletts kommen, schließt Caroline zu ihrem Sohn Aaron auf (\#2), sodass sie beide ihre letzten Schritte auf dem Weg zum Mammutskelett nebeneinander gehen (\#3). Nun stellt die Mutter den ,Schauwert‘ des Mammutskeletts durch ihr emphatisches „!WOW!“ heraus (Z. 1). Beide verlangsamen ihren Schritt und bleiben vor dem Exponat stehen.

- Von ihrer Kopfhaltung her zu urteilen, ist ihre gemeinsame Aufmerksamkeit auf den vor ihnen liegenden Raumbereich gerichtet, aber noch gibt es keine gemeinsame Orientierung. Zum Zeitpunkt von \#3 scheint Carolines Aufmerksamkeit auf den Stoßzähnen des Mammuts zu liegen, während Aaron eher in die links befindlichen Vitrinen schaut. Bei \#4 blickt Aaron zum Podest in der Bildmitte, während Caroline Aaron anschaut, wohl um seine Blickausrichtung zu überprüfen. Erst mit \#5 ist ein gemeinsamer Betrachtungsraum hergestellt. Der Junge zeigt auf eines der Exponate und nimmt durch das deiktische „SO“ auf dieses Objekt im geteilten Wahrnehmungsraum Bezug (Z. 4).

Die gemeinsame Konstruktion von Wissen setzt nun genau in dem Moment ein, in dem der gemeinsame Betrachtungsraum etabliert ist. Aaron kategorisiert das Objekt im gemeinsamen Gesichtsfeld als Vertreter der zoologischen Kategorie Elch und schreibt dieser Kategorie ein bestimmtes Aussehen als arttypisch zu (Z. 4). Allgemeiner formuliert: Ein Element, das zunächst im gemeinsamen Wahrnehmungsraum parasprachlich und gestisch zum Zentrum der Aufmerksamkeit gemacht worden ist, wird im Anschluss zum Gegenstand der interaktiven Konstruktion von Wissen. Ganz Ähnliches ist in der in 5.4 oben analysierten „Bartgeier“Szene zu beobachten (s.o. S. 381). Die Herstellung von Wissen in der Interaktion von Patrick und Nina beruht auf der vorgängigen Herstellung eines ganz spezifischen Betrachtungsstandpunkts. Erst nachdem dieser Betrachtungsstandpunkt hergestellt und vom Vater Patrick überprüft worden ist, werden Hypothesen zum Motiv für die Benennung des Bartgeiers entwickelt und überprüft.

In beiden Fällen ist also der Betrachtungsraum, der von den Besuchern hergestellt wird, identisch mit dem Raum, den sie zur Durchführung ihrer gemeinsamen Aktivität benötigen, der interaktiven Herstellung von gemeinsamem Wissen, das auf den Exponaten in der Vitrine (bzw. auf dem Podest) gründet.

Ist also die Herstellung des Betrachtungsraums im Rahmen des gemeinsamen Museumsbesuchs generell mit der Herstellung eines Handlungsraums gleichzusetzen? Nein, denn zum einen gibt es Fälle, in denen die für die kommunikative Nutzung der Ausstellung relevante Handlung nicht im Betrachten liegt, sondern

25 Das Mammut ist durch den Durchgang im Hintergrund links zu erreichen. 
beispielsweise im Manipulieren von Geräten, im Hören von Tierstimmen (s. „Zaunkönig“ in Kapitel 1) oder im Kommunizieren unter Anwesenden, das ein gegenseitiges Hören und Gehörtwerden voraussetzt. Hier kommt es darauf an, einen Raum $\mathrm{zu}$ konstruieren, der den Interaktionsbeteiligten Zugang zu den jeweils relevanten Handlungen ermöglicht: dem Zuhören, dem Bedienen von Knöpfen oder dem Berühren von Touchscreens. Betrachtungs- und Handlungsraum fallen hier nicht zusammen. Zum anderen gibt es aber Fälle, in denen der Handlungsraum temporär aus dem Betrachtungsraum in der Vitrine ,herausgezogen‘ wird.

Diesen letzteren Punkt möchte ich anhand von zwei Fällen exemplarisch untersuchen. Im ersten Fall - aus dem soeben untersuchten Video - zieht die Mutter Caroline die gemeinsame Aufmerksamkeit von der Vitrine ab, um den eigenen Körper zur Konstruktion von Wissen heranzuziehen. Im zweiten Fall ein Museumsbesuch einer Gruppe von Hortkindern - versucht eines der Kinder strategisch die Aufmerksamkeit aus dem in der Vitrine etablierten Betrachtungsraum abzuziehen, um damit eine eigene, auf Vorwissen basierende Behauptung gegen die visuelle Evidenz der Exponate in der Vitrine zu verteidigen.

Der erste Ausschnitt („Nägeli“) schließt unmittelbar an die Gesprächssequenz an, die wir weiter oben analysiert haben (,Knochen“).
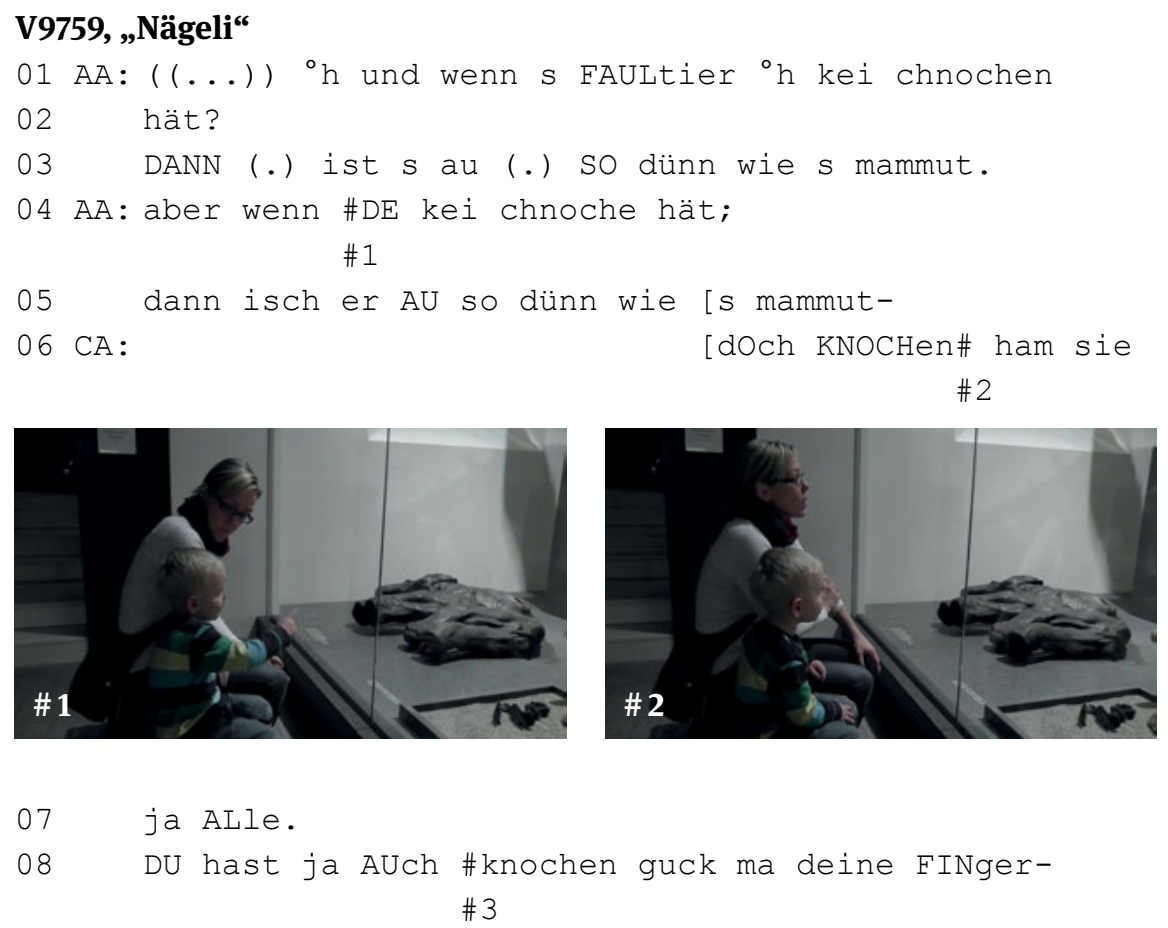

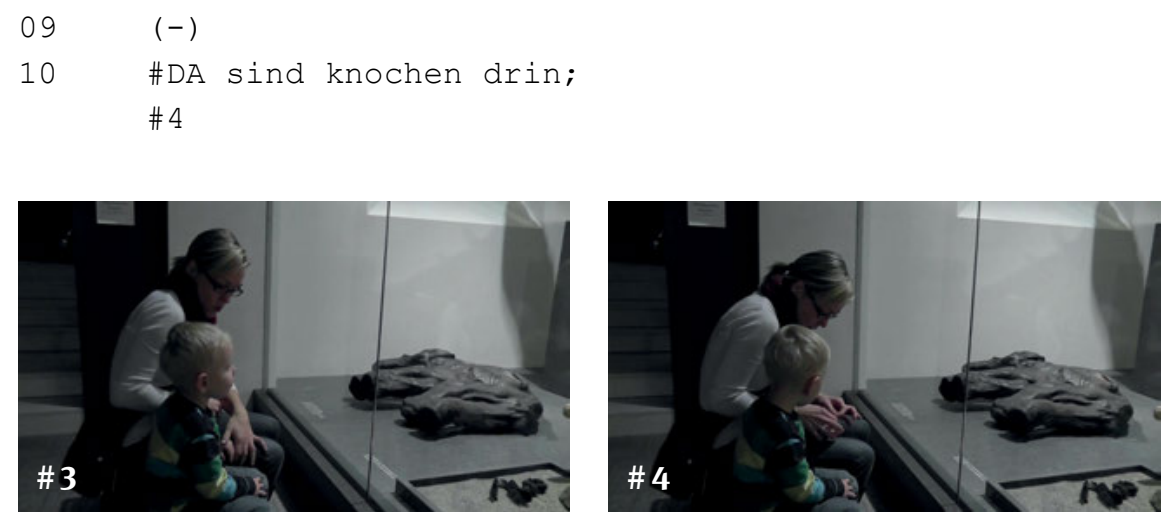
$d A$ wo $s$
\#mal da bi mir auch; $\# 5$ \#6
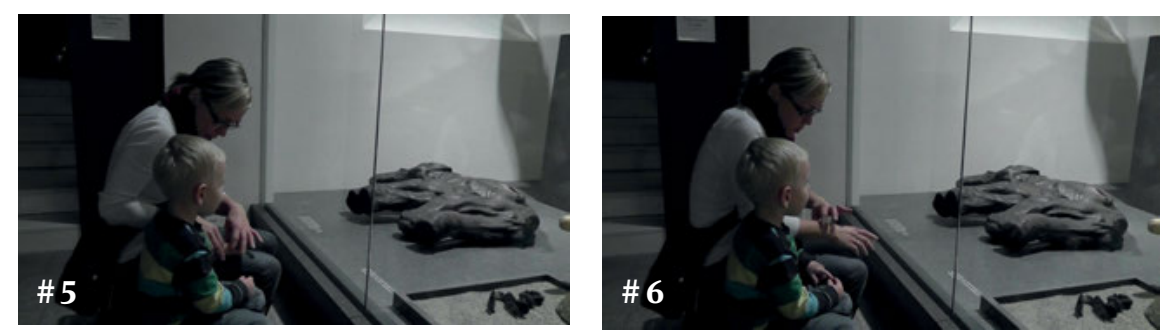
12 \#dA wo $\mathrm{s}$ \#HART is $[\langle<\mathrm{p}\rangle(\mathrm{da}$ sind knochen $)\rangle$.]
13 AA: \# \# [u (die/dicke) NÄ]geli?
\#7 \#8
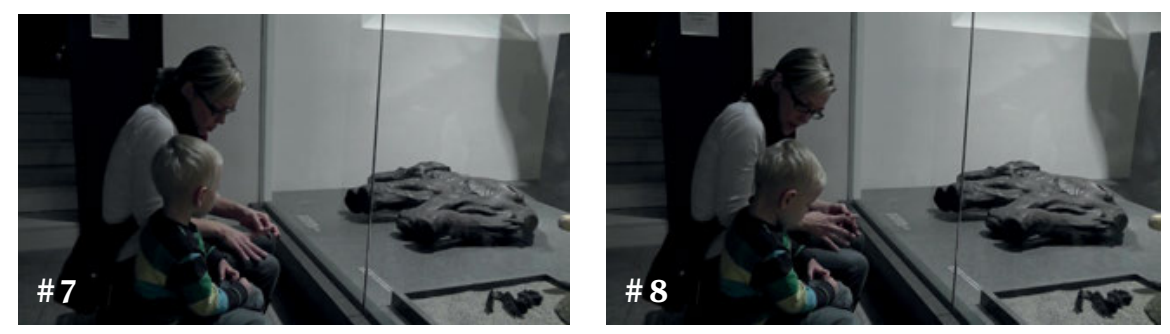

14 CA: die NÄ\#geli sind wieder $n$ bisschen ANders. \# 9 

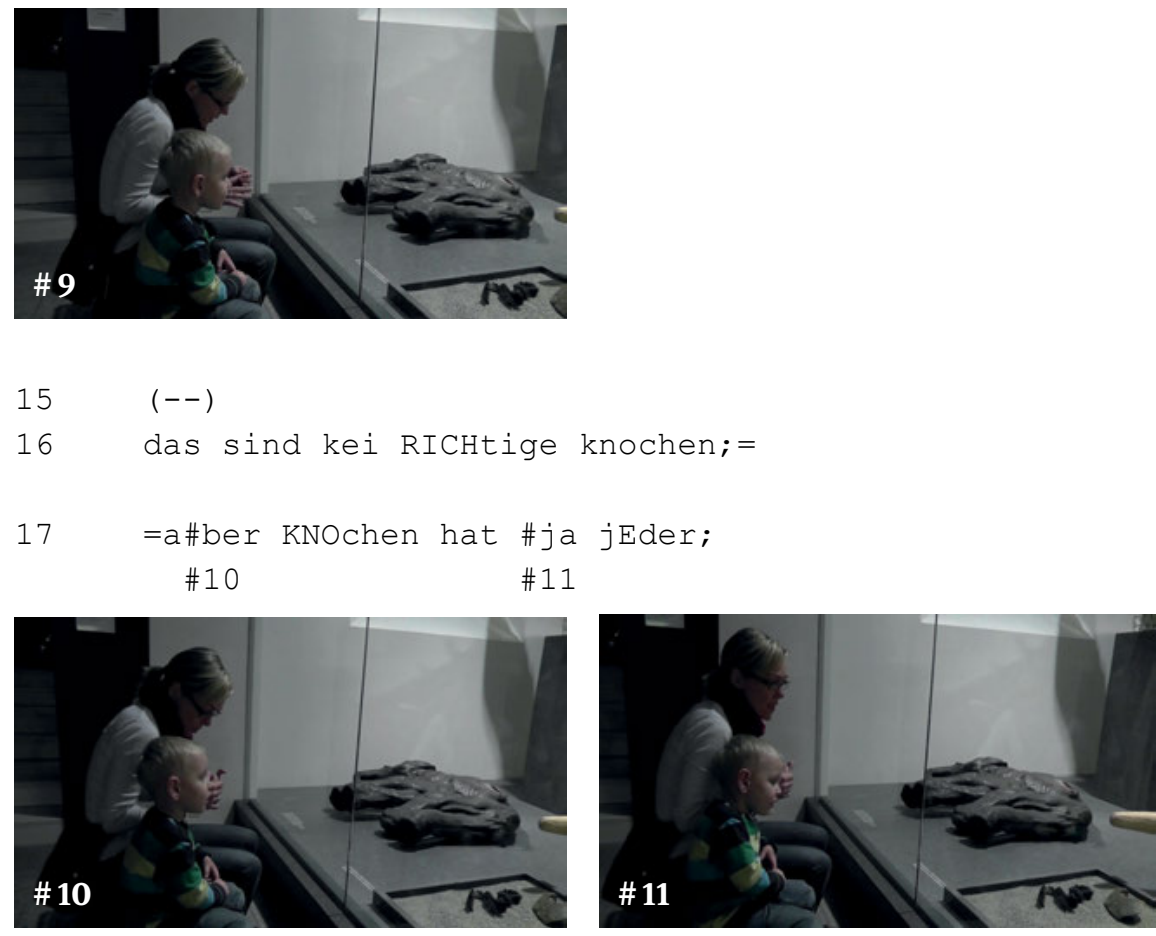

$18{ }^{\circ} \mathrm{h}$ sonst fëllt man zuSAMmen. ((räuspert sich))

Im Ausschnitt „Nägeli“ beginnen Caroline und Aaron, sich gemeinsames Wissen über Knochen und deren Funktion zu erarbeiten. Das Besondere an dieser Stelle ist, dass die Erarbeitung geteilten Wissens vorübergehend nicht mehr auf dem beruht, was die beiden Besucher in der Vitrine vorfinden. Vielmehr macht Caroline hier ihren eigenen Körper und den ihres Sohnes zu Demonstrationsobjekten, an denen sie ihre anatomische Behauptungen $\mathrm{zu}$ belegen versucht. ${ }^{26}$

- Zu Beginn des Transkriptionsausschnitts konzentrieren sich die beiden Besucher noch auf den gemeinsamen Betrachtungsraum in der Vitrine. Aaron stellt eine Schlussregel auf (Z. 1-3), die er mit einer Zeigegeste auf ein Exponat in der Vitrine bezieht (das Wisent, \#1), und Caroline korrigiert diese allgemeine Regel mit einer Allaussage, ebenfalls mit Blick auf das Wisent: „dOch KNOchen ham sie ja ALle.“ (Z. 6f., \#2).

26 Wie der Körper zum Objekt gemacht werden kann, an dem sprachlich formulierte Merkmale sichtbar gemacht werden können, haben Hindmarsh/Heath (2005: bes. 57-59) untersucht. 
- Dann aber überträgt sie ihre allgemeine Behauptung auf den Körper ihres Sohnes und vollzieht so einen Sprung von der allgemeinen zur persönlichen Ebene: „DU hast ja AUch knochen“ (Z. 8). Sie arbeitet jetzt daran, ihre allgemeine Behauptung über die Demonstration persönlicher Eigenschaften ihres Sohns zu belegen: Sie lenkt Aarons Aufmerksamkeit weg vom gemeinsamen Betrachtungsraum in der Vitrine und hin zu seinem Körper. Sie ergreift seine linke Hand (\#3) und drückt mit der Äußerung ,guck ma deine FINger-“ (Z. 8) nacheinander mehrere seiner Fingergelenke mit ihrem Daumen und Zeigefinger (\#4, \#5).

- Als nächstes lenkt sie Aarons Aufmerksamkeit auf ihre eigene Hand (\#6 bis \#8; „lug mal da bi mir auch“, Z. 11) und schreibt dieser ebenfalls die Eigenschaft zu, in ihrem Innern Knochen zu besitzen: „DA sind knochen drin; dA wo s HART is“ (Z. 10-12). Mit „dA wo s HART is“ gibt sie ihrem Sohn einerseits eine genauere ,Suchanweisung،, die diesem das Nachvollziehen der mit dem Ergreifen des Fingers bzw. ihrer Hand vollzogenen Referenz erleichtert. Andererseits aber schreibt sie gleichzeitig Knochen eine allgemeine Eigenschaft zu: Sie sind „HART“. So nutzt Caroline ihren Körper und den ihres Sohns, um allgemeine Behauptungen über einen in der Ausstellung präsentierten Wissensaspekt (Knochen als Teile des anatomischen Aufbaus von Lebewesen) an ihren eigenen Körpern sinnlich erfahrbar zu machen.

- Aaron übernimmt die von Caroline initiierte Ausrichtung des Aufmerksamkeitsbereichs (zu sehen in \#8) und schaut auf seine Hand herab, während er nach dem ,Knochen-Status“ der Fingernägel fragt (,u (die/dicke) NÄgeli?““, Z. 13). Der eigene Körper wird also zum Objekt, dessen Betrachtung und Berührung zur Konstruktion von Wissen über einen zoologischen Sachverhalt beiträgt.

- Caroline antwortet nun auf die Frage nach den Fingernägeln, indem sie Gemeinsamkeiten und Unterschiede zu den Knochen beschreibt (,sind n bisschen ANDERS“, „kei RICHtige knochen“, Z. 14, 16). Dabei bleibt ihr Blick auf ihre eigene Hand gerichtet (\#9, \#10). Diese Ausrichtung des Blicks verändert sie aber in dem Moment, in dem sie mit der allgemeinen Aussage ,aber KNOchen hat ja jEder; ${ }^{\circ} \mathrm{h}$ sonst fällt man zuSAMmen.“ (Z. 17f.) wieder auf die kurzzeitig verlassene Ebene der allgemeinen Aussagen zu Knochen zurückkehrt: Ihr Blick wandert zurück in die Vitrine (\#11). Aaron hat schon während ihrer Erklärung den Blick zurück in die Vitrine gewendet (\#9). Damit ist die gemeinsame Aufmerksamkeit der beiden Besucher wieder auf das Innere der Vitrine fokussiert.

Das erfolgreiche Herstellen von Beziehungen zwischen der Person des Besuchers und den Gegenständen der Ausstellung ist von der psychologischen Forschung zum Lernen im Museum als wichtiger Faktor für das Gelingen des Lernens im Museum beschrieben worden (s. dazu Hooper-Greenhill 2008 oder Harms/ 
Krombaß 2008). In dem soeben untersuchten Beispiel „Nägeli“ können wir im Detail sehen, wie die Verbindung von Person und Ausstellung von den Interaktionspartnern vollzogen werden kann und wie sie den Übergang von verallgemeinernden und partikularisierenden Aussagen an den Exponaten mit personalisierenden Aussagen verbinden, die sie an ihrem eigenen Körper vornehmen.

In dem hier verfolgten Argumentationsgang ist der Ausschnitt „Nägeli“ interessant, weil er zeigt, wie die beiden Besucher den gemeinsamen Wahrnehmungsund Handlungsraum vorübergehend aus der Vitrine heraus verlagern können. Die geteilte visuelle Aufmerksamkeit liegt damit nicht mehr auf dem, was für die Besucher im Ausstellungsraum arrangiert worden ist, sondern in einem Bereich vor der Vitrine. Dort nutzen die Besucher ihre Körper, um an ihnen Erfahrungen zu machen, die das Ausgestellte mit der eigenen Person in Beziehung setzen und so zum Verständnis des Ausgestellten beitragen. Der museale Betrachtungsraum ist aber nicht vollends aufgehoben, sondern lediglich zeitweilig suspendiert: die Besucher erhalten mit ihren auf dem kleinen Bänkchen ruhenden Unterkörpern die Orientierung auf das Vitrineninnere aufrecht, und die Reaktivierung des Betrachtungsraums bedarf keinerlei auffälliger Anstrengungen (lediglich einer kurzen Veränderung der Kopfhaltung) und keiner expliziten Aushandlung.

Der Betrachtungsraum, den Caroline und Aaron hier vor der Mammutvitrine hervorgebracht haben, und der Handlungsraum, in dem die beiden Besucher an ihren Körpern etwas über die Härte von Knochen lernen, fallen an dieser Stelle der Interaktion auseinander.

In dem zweiten Beispiel kommt es ebenfalls zu einer Abwendung vom in der Vitrine etablierten gemeinsamen Betrachtungsraum und $\mathrm{zu}$ einer Verlagerung des Handlungsraum in den Bereich vor der Vitrine. Dieses Mal allerdings geht es nicht darum, den Körper der Besucher in die Wissenskonstitution einzubeziehen. Vielmehr stellt die Verlagerung des Handlungsraums aus der Vitrine heraus einen strategischen Schritt dar, mit dem eine der Interaktionsteilnehmerinnen versucht, die Gültigkeit einer Behauptung im Gespräch durchzusetzen, die sich auf ihr Vorwissen, und gerade nicht auf das in der Vitrine Sichtbare stützt.

Der folgende Ausschnitt („Eichhörnchen“) dokumentiert einen Ausschnitt aus dem Museumsbesuch einer Gruppe von vier Kindern („Anna“, AN; „Chrigu“, CH; „Didier“, DI; und „Emil“, EM) und einer Hort-Betreuerin („Barbara“, BA), aus dem wir schon weiter oben einen Ausschnitt untersucht haben (Ausschnitt „Weiter“, 5.2.4).27

27 Dieses Video ist bereits in Kesselheim (2012) untersucht worden. 


\section{V3210, „Eichhörnchen“}

01 BA: JÄ (.) [was isch dänn das] da,

02 AN: [chann die bisse, ]

03 BA: das da(.) \#känned_er jetz; [ ( ) ]

$04 \mathrm{CH}$ :

[EICH] hörnchen-

\# 1

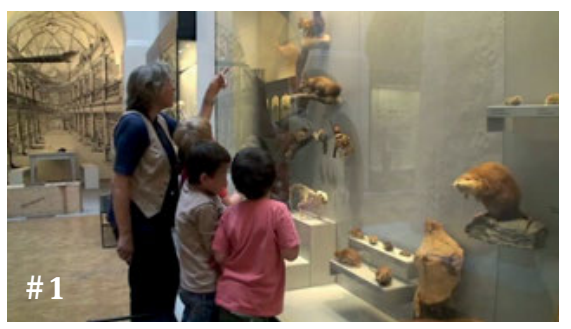

05 BA: das SIND eichhörndli-=hä,

06 (-) und die_sch da unne isch aU es EICHhörndli aber das sind

07 glaub afrikanischi-

08 (.) amerikanischi sind die [grAUe; ]

$09 \mathrm{EM}:$

10 AN: \#die sind \#RIsig-

\#2 \#3
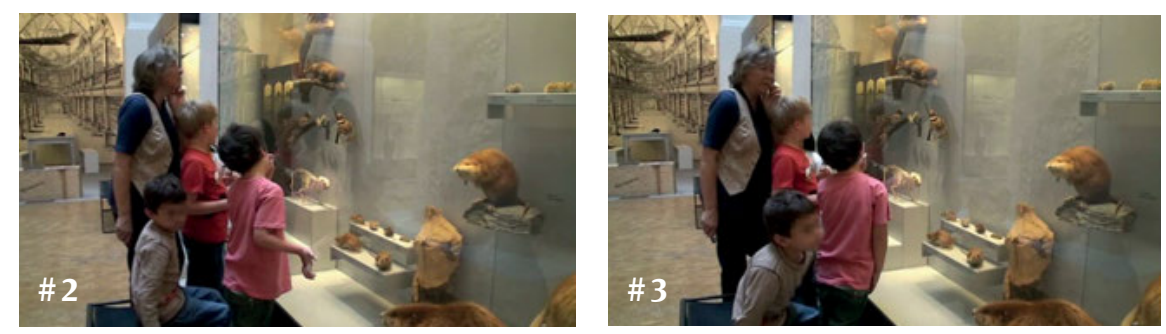

11 (.) die chönnd \#RIsig werde; \# 4

12 BA: !NEI! (.) \#nid grösser weder SO; \# 5 

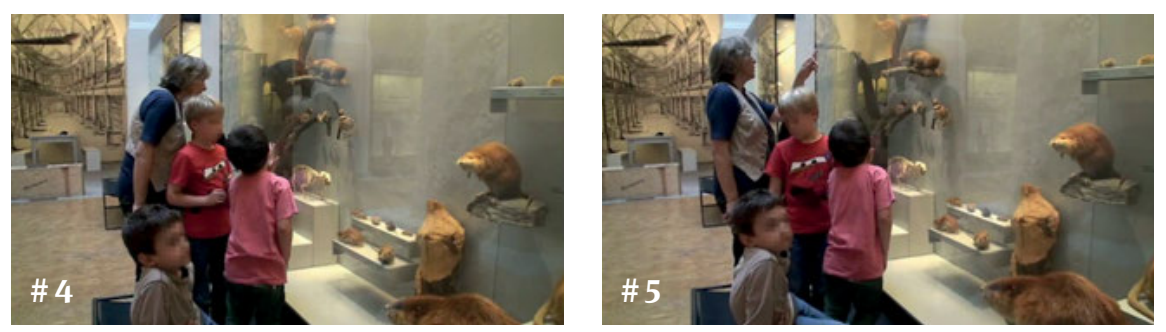

13 (.) nöd vil GRÖSser;

14 (-) da z_zÜri häts au söttig\# (.) det wo_n_ich \# 6

15 wohne [häts au so (eichhörnli) (so)- ]

16 AN: [ABer (.) ameri\#kAnischi chönnd] so gross werde; \# 7
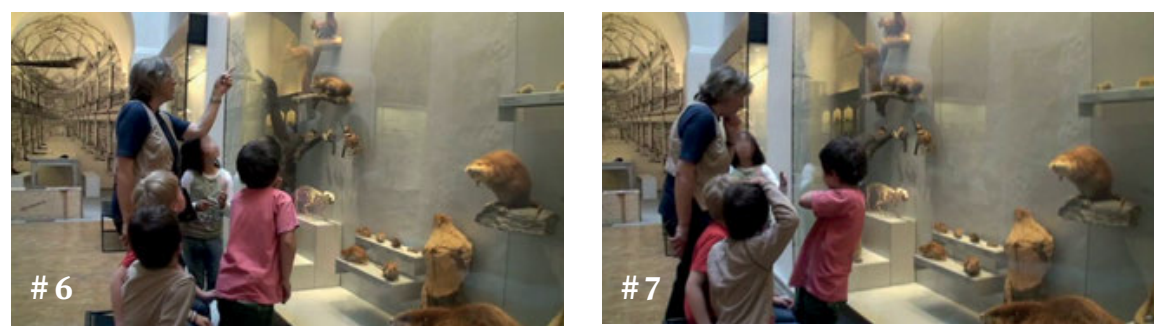

17 BA: \# (.)Ah ja (kla:r;)\# \# 8 \# 9
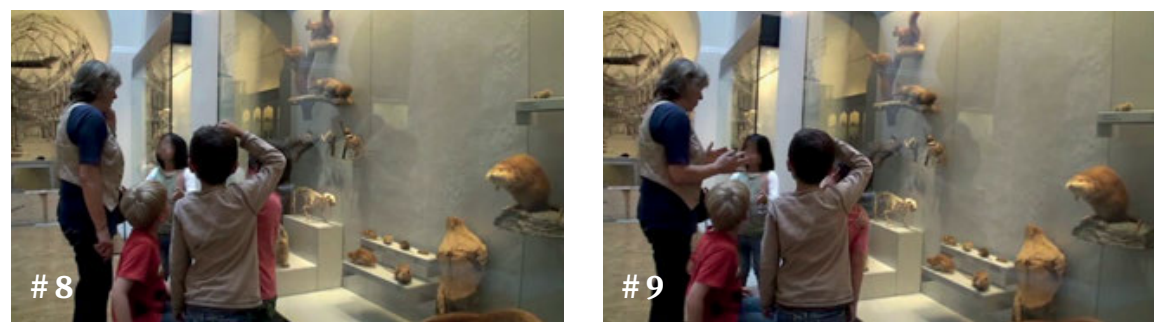

18 (-) \#die sind nöd so gross; \# 10 


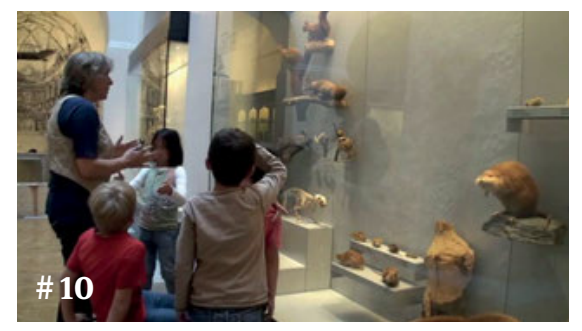

19 AN: doch die chönnd esO GROSS werde;

20 BA: ach_han_i noch nie ( ) villicht,

21 (.) hesch denn du e GROSses gseh [mal] in amerika-

$22 \mathrm{AN}$ :

$[\mathrm{JA}-$ ]

Im Ausschnitt „Eichhörnchen“ gelingt es dem Mädchen Anna (AN) für kurze Zeit, die geteilte Aufmerksamkeit der Gruppe aus der Vitrine hinaus zu lenken und auf den Bereich vor der Vitrine zu konzentrieren, wo sie eine Behauptung gestisch veranschaulicht, die sie auf ihr Vorwissen abstützt.

- Zu Beginn des Transkripts ist die Aufmerksamkeit der Gruppe in der Vitrine konzentriert. Barbara weist mit Hilfe des deiktischen „das da“ und einer gleichzeitigen Zeigegeste (\#2) auf ein Eichhörnchenexponat in der Vitrine hin. Das Exponat im gemeinsamen Wahrnehmungsraum der Gruppe verbindet sie mit dem Aspekt des Wissens, indem sie das Vorliegen eines bestimmten Vorwissens behauptet: „das da (.) känned_er jetz;“ (Z. 3). Barbaras Begleiter treten in diese Wissenskommunikation ein und identifizieren das Exponat in Begriffen der zoologischen (Alltags-)Terminologie: „EICHhörnchen“ (Z. 4). Barbara bestätigt diese Identifizierung und nutzt sie als Ausgangspunkt, um verschiedene Arten dieser Gattung, die in der Vitrine repräsentiert sind, zueinander in Bezug zu setzen: „das SIND eichhörndli-=hä, (-) und die_sch da unne isch aU es EICHhörndli aber das sind glaub afrikanischi- (.) amerikanischi sind die GRAUe;“ (Z.6-8).

- Nun kommt es zu dem hier interessierenden Ereignis. Das Mädchen Anna behauptet, dass amerikanische Eichhörnchen ,riesig‘ sind bzw. werden können. Während sie das Wort riesig ausspricht, wendet sie sich von der Vitrine ab und blickt zu Barbara (wie man in der Spiegelung der Glasfront sehen kann, \#5, \#6). Diese Abwendung von der Vitrine ist, wie wir gleich sehen werden, nicht zufällig, sondern lässt sich in Zusammenhang mit Annas Argumentation sehen.

- Barbara bestreitet Annas Behauptung: „!NEI!. nid grösser weder SO;“ (Z. 12). Dabei behält sie ihren visuellen Fokus auf die Vitrine bei. Sie zeigt und blickt auf das Exponat (\#7). Erneut setzt BA also bei dem in der Vitrine Sichtbaren 
an, und knüpft an dieses Sichtbare ein Element ihres persönlichen Vorwissens: „da z_ZÜri häts au söttig (.) det wo_n_ich wohne häts au so (eichhörnli) (so)-“ (Z. 14f.). ${ }^{28}$ Annas Blick folgt Barbaras Zeigegeste, so dass sie das durch „SO“ Behauptete visuell überprüfen kann, und die Position der anderen Kinder lässt erkennen, dass auch für sie die Eichhörnchenexponate das Zentrum des gemeinsamen Betrachtungsraums sind.

- Anna beharrt auf ihrer Behauptung („ABer (.) amerikAnischi chönnd sO gross werde;“, Z. 16). Sie breitet ihre Arme aus, um die Größe der amerikanischen Eichhörnchen anzudeuten, tritt gleichzeitig zwei Schritte zurück und dreht sich von der Vitrine weg (\#8). Sie steht nun so, dass sie das ausgestopfte Eichhörnchen nicht mehr sehen kann. Und wenn sie Anna ansehen, und genau das hat Anna durch ihre Geste notwendig gemacht, dann können auch sie nicht mehr gleichzeitig in die Vitrine sehen.

- Anna gelingt es mit dieser Aktion, den gemeinsamen Betrachtungsraum, der zuvor im Innern der Vitrine lag, aufzuheben und die Aufmerksamkeit der anderen auf ihre Person zu lenken (\#9). Damit ist es ihr gelungen, einen gemeinsamen Wahrnehmung- und Handlungsraum außerhalb der Vitrine zu erzeugen und den Bezug der Wissenskonstruktion auf die Vitrine zu unterbrechen. Doch schon mit ihrer nächsten Äußerung lenkt BA die Aufmerksamkeit der Gruppe wieder zurück in die Vitrine.

- Barbara ,überträgt‘ Annas vor der Vitrine ausgeführte Geste auf das Exponat, um ihr Zutreffen zu überprüfen, und zwar indem sie Annas Geste nachahmt und dann ihren Blick von ihren Armen in die Vitrine hineinlenkt (\#9, \#10). Auf diese Weise lenkt sie die Aufmerksamkeit der Gruppe wieder zurück in die Vitrine. Hier ist ihre abschließende Behauptung „die sind nÖd SO gross;“ wieder verankert (Z. 18).

Zusammenfassend kann man sagen: Anna, die Wissensbestände durchzusetzen versucht, die auf ihrer persönlichen Erfahrung mit lebenden Eichhörnchen beruhen, und gerade nicht auf dem in der Vitrine Sichtbaren, arbeitet sichtbar daran, die visuelle Bezugnahme auf den Innenraum der Vitrine zu blockieren und einen eigenständigen Handlungsraum vor der Vitrine aufzubauen, in den sie die gemeinsame Aufmerksamkeit lenkt. Barbara dagegen, die die Konstruktion von Wissen auf dem in der Vitrine Wahrnehmbaren aufbaut, versucht, die Inter-

28 Musterhaft für die Wissenskommunikation vor der Vitrine ist wieder die Verbindung von Wahrnehmungsraum (in dem die Behauptungen mithilfe von Deiktika wie „da“ oder „so“ verankert werden) und Aussagen über situationsüberschreitende Sachverhalte, hier: über Wissen zur Verbreitung der in der Vitrine repräsentierten Gattung. 
aktionspartner dazu zu bewegen, einen gemeinsamen musealen Betrachtungsraum zu konstruieren, in dessen Zentrum die Exponate in der Vitrine stehen. Das ist für Barbaras Argumentation funktional, denn für die museale Form der Wissenskonstruktion benötigt sie den Blick auf die Exponate. Zwar ,torpediert` Anna hier die visuelle Bezugnahme auf die Exponate, von der - wie wir weiter oben gezeigt haben - die lokale Herstellung von Wissen im gemeinsamen Museumsbesuch charakteristischerweise ausgeht. Das heißt aber nicht, dass sich Anna hier aus der musealen Wissenskommunikation ausklinkt. Anna nutzt den Raum vor der Vitrine, der durch die Körper der Interaktionsbeteiligten gebildet wird, in einer Weise, die für den gemeinsamen Museumsbesuch durchaus typisch ist. Sie arbeitet mit an der gemeinsamen Konstruktion zoologischen Wissens, konkret: an der Bestimmung der Größe von Eichhörnchen. Wenn aber im Raum vor der Vitrine eine Handlung realisiert wird, die für den gemeinsamen Museumsbesuch relevant ist, haben wir es hier mit einem musealen Handlungsraum außerhalb der Vitrine zu tun, der sich nicht mit dem museumstypischen Betrachtungsraum deckt. Die beiden Räume fallen also im Museum, wie dieser Fall belegt, nicht automatisch zusammen.

Die Analyse der Ausschnitte „Nägeli“ und „Eichhörnchen“ hat erbracht: Wenn es auch stimmt, dass Handlungen, die für den gemeinsamen Museumsbesuch relevant sind (das Betrachten der Exponate, das Lesen der Vitrinentexte usw.), häufig im gemeinsamen Betrachtungsraum in der Vitrine ablaufen, so kann man Betrachtungsraum und Handlungsraum doch nicht einfach gleichsetzen. In den beiden soeben untersuchten Beispielen verlagern Besucher das relevante Geschehen aus der Vitrine heraus in den Bereich vor die Vitrine und lenken die geteilte Aufmerksamkeit auf ihre eigenen Körper. Im ersten Beispiel hat diese Verlagerung die Funktion, die Allgemeingültigkeit einer Aussage zu demonstrieren, die von Beobachtungen an den Exponaten ausgegangen ist, beziehungsweise allgemeine Behauptungen mit persönlichen (Körper-)Erfahrungen der Besucher $\mathrm{zu}$ verbinden. Im zweiten Beispiel ist mit der Verlagerung der Aufmerksamkeit die Funktion verbunden, die Basis der lokalen Wissenskonstruktion zu verschieben: von den Exponaten im Vitrineninneren zum persönlichen Vorwissen einer Interaktionsteilnehmerin. Beide Male setzen die Besucher die exponatbasierte Wissenskonstruktion außer Kraft, die weiter oben als museumstypisch beschrieben worden ist, ohne dass es Hinweise darauf gäbe, dass sie ihre auf den Raum vor der Vitrine bezogenen Handlungen als eine Unterbrechung ihres Museumsbesuchs auffassten. Vielmehr setzen die Gesprächsabschnitte, in denen die Aufmerksamkeit aus der Vitrine gezogen worden ist, die Wissenskonstruktion fort, die von den Exponaten in der Vitrine ausgegangen ist.

Der museale Handlungsraum darf also nicht mit dem Betrachtungsraum in der Vitrine gleichgesetzt werden, selbst wenn - wie wir in Kapitel 3 gesehen haben - im 
Raum vieles dafür getan wird, dass wir uns bei der kommunikativen Nutzung des Ausstellungsraums ganz auf die visuelle Nutzung der Vitrinen und ihrer Exponaten konzentrieren. Der Handlungsraum umfasst zweifellos auch den Bereich vor der Vitrine, wo er im Sinne eines Kendon'schen „o-space“ (Ciolek/Kendon 1980: 258) zwischen den Körpern der miteinander interagierenden Besucher konstruiert wird. Denn sobald man den Ausstellungsraum nicht alleine, sondern zusammen mit anderen nutzt, gehört zur kommunikativen Nutzung nicht nur das Betrachten der Exponate, sondern - individuell unterschiedlich ausgeprägt, aber in meinen Daten niemals gänzlich fehlend - der sprachliche Austausch über die Exponate. Und dazu ist es nötig, einen gemeinsamen Interaktionsraum aufzubauen, in dem die Besucher sich zumindest gegenseitig hören, in aller Regel aber auch sehen können.

Dass sich die Besucher beim gemeinsamen Gang durch das Museum in der Regel nah beieinander aufhalten, hat seinen Grund also nicht nur in der Notwendigkeit, die Herstellung eines gemeinsamen Betrachtungsraums zu organisieren, wenn das auch der in den Daten bei Weitem der auffälligere Aspekt ist. Die gegenseitige Nähe ist auch erforderlich, um sich bei der gemeinsamen Wissenskonstruktion hören zu können. Hieraus ergibt sich die typische Ausprägung des Handlungsraums für den gemeinsamen Museumsbesuch. Ein Teil des Handlungsraums reicht weit in die Vitrine hinein: Hier geschieht ein Gutteil der Konstruktion fachlichen Wissens, die wir im Videokorpus beobachten konnten. In diesem Bereich kommen der museale Betrachtungsraum und der Handlungsraum zur Deckung. Es gibt aber noch einen weiteren Teil des Handlungsraums. Dieser wird zwischen den Körpern der Besucher etabliert und befindet sich somit außerhalb der Grenzen des musealen Betrachtungsraums. Wenn er auch in den Analysen der Ausschnitte „Nägeli“ und „Eichhörnchen“ klar zutage getreten ist: Die meiste Zeit ist dieser Handlungsraum vor der Vitrine vor allem ein akustischer Raum.

\subsubsection{Betrachten, Wissen konstruieren, Unterhaltung}

Zweifellos kann man sich einen gemeinsamen Museumsbesuch vorstellen, bei dem die Besucher ihre gemeinsame Aktivität im Ausstellungsraum auf das gemeinsame Betrachten der Exponate beschränkten, ohne interaktiv Wissen zu konstruieren. Wie wir in Kapitel 3 festgestellt haben, wird das Betrachten der Exponate durch zahlreiche unterschiedliche Erscheinungsformen im Ausstellungsraum hochgradig erwartbar gemacht. Und in der Tat haben wir in 5.3 gesehen, wie viel Aufwand die Besucher darauf verwenden, zu einer gemeinsamen, an der Maximierung der wahrgenommenen Details orientierten Betrachtung der Exponate zu gelangen (und Kindern dieses ,richtige‘ Sehen als Teil der gemeinsamen sozialen Praxis zu vermitteln). Auch wenn also ein Museumsbesuch 
durchaus denkbar wäre, der sich nur auf ein koordiniertes Betrachten beschränkte, findet sich in meinem Videokorpus kein solcher Fall. Zwar gibt es durchaus Videos, auf denen sich die Besucher über längere Zeit hinweg schweigsam den Exponaten widmen. Doch schaut man sich jeweils die gesamte Aufnahme an, findet man immer Phasen der gemeinsamen Wissenskonstitution (die typischerweise eng auf das im Ausstellungsraum Betrachtete bezogen ist).

Betrachten und Wissen konstruieren scheinen mir also die zwei ,Grundpfeiler der sozialen Praxis des gemeinsamen Museumsbesuchs zu sein, und mit beiden Aktivitäten befinden sich die Besucher im Einklang mit den im Raum als dominant signalisierten Funktionen der Ausstellung (s.a. 5.4.2.1). Die Rekonstruktion der museumsspezifischen Herstellung von Betrachtungs- und Handlungsraum in diesem und dem vorausgehenden Analyseabschnitt erfasst damit einen Gutteil der Spezifik der sozialen Praxis des gemeinsamen Museumsbesuchs.

Ein Aspekt ist aber bisher außer acht gelassen worden. Denn er erscheint mir in den von mir untersuchten Museen deutlich weniger relevant $\mathrm{zu}$ sein als das Betrachten und das raumbasierte Herstellen von fachlichem Wissen. Und dennoch scheint er immer wieder in meinen Daten auf: der gemeinsame Museumsbesuch als unterhaltsame Aktivität, die eine - bisweilen starke - emotionale Beteiligung auslöst. Besucher signalisieren sich während ihres Museumsbesuchs, dass ihre gemeinsame Aktivität Emotionen in ihnen auslöst, die von ehrfürchtiger Ergriffenheit oder Staunen bis hin zu Freude oder Belustigung reichen können (Allen 2002: 276f. et passim spricht hier von ,affective talk“, einen Überblick zur Erforschung der Rolle von Emotionen für das museale Lernen gibt Thoma 2009: Kap. 3.2.2). Oder sie signalisieren sich, dass sie gerade eine ästhetische Erfahrung gemacht haben (vgl. vom Lehn/Heath 2007a, Heath/vom Lehn 2004). Das Bewerten, ein ,außerordentlich prominenter Zugzwang“ in der Interaktion in Kunstmuseen (Hausendorf 2007: 33, Hausendorf/Müller 2016: 6-9), lässt sich in meinem Korpus dagegen nur punktuell nachweisen: in den oben zitierten Darstellungen ästhetischer Erfahrungen oder in allgemeinen Charakterisierungen der Ausstellungsstücke als „krass“, oder „megacool“ (beide Zitate: V2910). Einige Beispiele aus meinem Korpus sollen die große Spannweite der von den Besuchern dargestellten Emotionen und Bewertung der Erfahrungen illustrieren:

- Ästhetik: „lueg emal samuel das da isch de FUCHS, (-) e SCHÖnes fell-“ („Otto“ in V8158), „SCHÖ::N,“ (,Sybille“ in V3853);

- Überwältigung: „so_öppis söllt mer ma natU i de naTUR usse gseh.“(„Roman“ in V3853);

- Staunen: „da stuunsch_de“ - „SUper“ („Urs“ und „Doris“ in V0344); „das isch UNgehür;=hä?“ („Otto“ in V8158);

- Überraschung: „!heieiEI!; was isch denn DAS da.“ („Otto“ in V8158); 
- Spaß: neben häufigen Belegen für redebegleitendes Lachen: „lUg emal was der für luschtigi OHre hät;“(„Patrick“ in V8816), „lueg da häts nämlich au no öppis lässigs; “(„Susanne“ in V8516);

- Entzücken: „DÄNK s isch scho_nes HERzigs vÖgeli“ („Änni“ in V2910); „JÖ:-“ („Chrigu“ in V3210).

Diese Darstellungen von emotionaler Beteiligung oder ästhetischen Erfahrungen sind sequenziell eng mit der Betrachtung der Exponate und der raumbasierten Herstellung von Wissen verwoben. Das dokumentiert der folgende Ausschnitt (,Biber“) aus dem Museumsbesuch der Hortgruppe, der wir schon in „Weiter“ (5.2.4) und „Eichhörnchen“ (5.4.3) begegnet sind (s. dazu auch Kesselheim 2012).

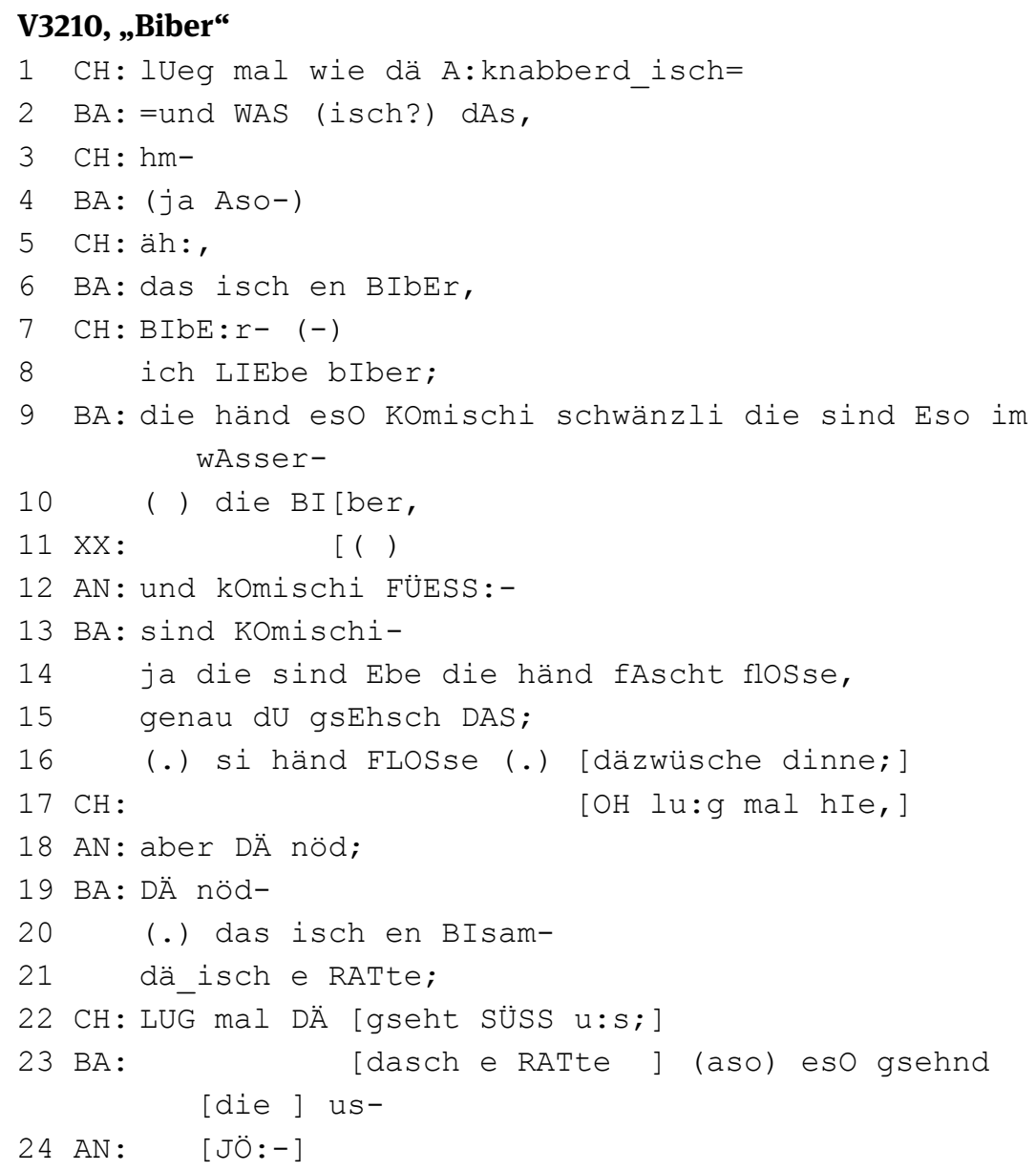


Hier finden wir eine ganze Reihe von impliziten und expliziten Formen der Darstellung emotionaler Beteiligung sowie von Bewertungen des Betrachteten:

- Chrigus begeisterter Ausruf „lUeg mal wie dä A:knabberd_isch“ (Z. 1),

- $\quad$ sein emphatisches „BIbE:r- (-) ich LIEbe bIber;“ (mit Überlautung des eigentlich unbetonen Schwa, Z. 7f.),

- Barbaras und Annas gemeinsame Zuschreibung einer ,komischen‘ Qualität von Körperteilen des Bibers (Z. 9-15),

- Chrigus erneutes lautes, gedehntes Rufen, mit dem er die Aufmerksamkeit auf ein neues Exponat lenkt („OH lu:g mal hIe,“ Z. 17) und schließlich

- seine explizite Bewertung der Bisamratte als „SÜSS“ (Z. 22), bei der er von Anna sekundiert wird (,JÖ:-“, Z. 24).

Interessant ist nun, dass diese Darstellungen emotionaler Beteiligung von ihrer Begleiterin Barbara mit Aspekten der Wissenskonstruktion verzahnt werden.

- Chrigus ersten emphatischen Ausruf führt Barbara über in die Frage nach der Einordnung des Exponats in die einschlägige zoologische Kategorie: Chrigu: „lUeg mal wie dä A:knabberd_isch“, Barbara, in besonders engem zeitlichen Anschluss: „=und WAS (isch?) dAs,“(Z. 1f.);

- an Chrigus ,Liebesbekenntnis‘ knüpft sie eine Beschreibung eines Körperteils und eine Information zum typischen Habitat des Bibers an (Z. 9);

- in ihre Charakterisierung von Körperteilen des Bibers als ,komisch“ mischt sie die Beschreibung zoologischer Fakten (zum Biberschwanz, zum Wohnraum Wasser und zu den speziell geformten Füßen), mit denen sie implizit an eine zoologische Theorie der Anpassung an den bevorzugten Lebensraum anspielt (s. dazu genauer Kesselheim 2012)

- $\quad$ und schließlich reagiert sie auf Chrigus Charakterisierung der Bisamratte als „SÜSS“ (Z. 22), indem sie ihre Einordnung des Exponats in eine zoologische Kategorie (,e RATte“, Z. 23) vornimmt ${ }^{29}$ und mitteilt, dass sie jetzt etwas über die Gattung (Plural „die“) gelernt hat.

Die lokale Konstruktion von Wissen und die Darstellung von Staunen, Begeisterung und ähnlichen Emotionen mehr sind in diesem Beispiel eng miteinander verflochten. Hier - und in einer Reihe weiterer Aufnahmen, für die der Ausschnitt „Biber“ repräsentativ ist - stehen die Kundgebung von Staunen und Begeiste-

29 Wie schon zuvor gesagt, spielt es keine Rolle, dass es sich hier nicht um die zoologisch korrekte Kategorie handelt. Wichtig ist hier, dass und wie Barbara das Exponat für den objektbasierten Wissenserwerb nutzt. Dass die Wissensvermittlung aus der Sicht von Zoologen hier scheitert, ist primär ein ausstellungspraktisches Problem. 
rung einerseits und die Konstruktion von fachlichem Wissen andererseits in einer Art Konkurrenzverhältnis, und die erwachsene Begleitperson lenkt die von den Kindern signalisierte emotionale Beteiligung immer wieder auf Aspekte um, die für den Erwerb von Wissen nutzbar zu machen sind.

In einer anderen Gruppe von Aufnahmen dagegen ist das Verhältnis von (signalisierten) Emotionen und Wissenskonstruktion ein anderes. Dort signalisieren die Erwachsenen Begleiterinnen oder Begleiter selbst eine intensive emotionale Beteiligung und tragen so zur Definition der gemeinsamen Besuchsaktivität als etwas Anregendem, Lustigen oder Lustvollen bei. Ein Beispiel hierfür ist der folgende Ausschnitt („Alpenschneehuhn“), in dem zwei Mütter mit ihren zwei Kleinkindern („Susanne“, SU, mit ihrem Sohn „Julius“, JU, sowie „Petra“, PE, mit dem präverbalen „Lars“, LA) das zoologische Museum besuchen. Die Forschung zum Familien-Lernen im Museum hat beschrieben, dass Eltern so das „involvement“ ihrer Kinder mit der Ausstellung erhöhen und die Beschäftigung ihrer Kinder mit den Exponaten verlängern können (Ash 2002, Kelly 2007). Die Gruppe hört sich an einer Audiostation Vogellaute an. Beide Mütter haben ihren Kindern die Hörer der Audiostation ans Ohr gehalten, woraufhin diese sich die Laute des Alpenschneehuhns anhören. Sobald das Abspielen des Lauts beendet ist, nimmt Susanne den Hörer von Julius` Ohr und lacht ihn an:

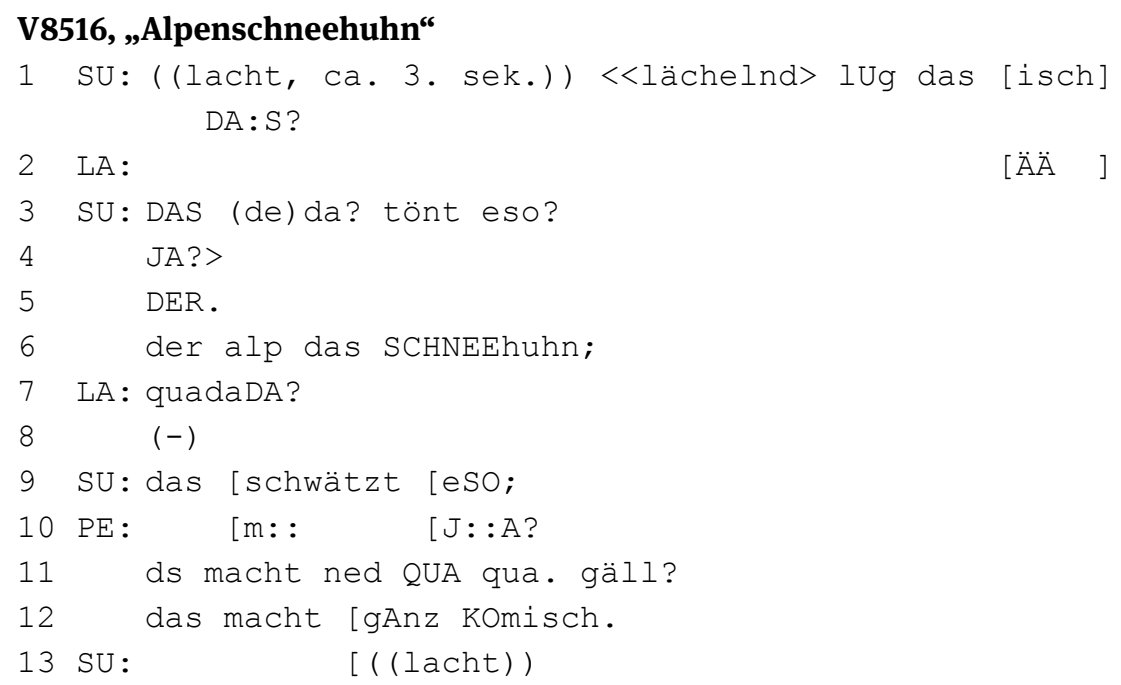

Das lange Lachen Susannes, das langsam in einen lächelnden Tonfall mündet, den Susanne fast bis zum Ende ihres Beitrags beibehält, macht aus der ganz gewöhnlichen Aktivität des Abhörens einer CD etwas besonders Lustiges. Auch werden die Schneehuhnlaute mit deutlicher Betonung als „gAnz KOmisch“ 
charakterisiert, und diese Charakterisierung durch Petra löst ein erneutes Lachen von Susanne aus. Auffällig ist schließlich auch die Vermenschlichung des Schneehuhns durch das Verb schwätzen, die meines Erachtens hier expressive Funktion hat. Mit anderen Worten: Petra und Susanne unterstützen sich gegenseitig dabei, die gemeinsame Nutzung der Vitrinen für ihre kleinen Kinder als etwas Lustiges, Spaß Machendes darzustellen.

Im Ausschnitt „Alpenschneehuhn“ ist die Signalisierung von Spaß eindeutig auf die museale Wissensvermittlung bezogen: Es geht Susanne und Petra offensichtlich darum, ihren kleinen Kindern die Nutzung der Audiostation und das Betrachten des Schneehuhnexponats schmackhaft zu machen. Aber Spaß und spielerische Momente können immer wieder auch den Status selbstständiger Sequenzen erhalten, wofür es in meinem Korpus eine Reihe von Belegen gibt.

Die nächsten beiden Ausschnitte - beide aus dem Museumsbesuch von Caroline und Aaron (s.o.) - dokumentieren solche Fälle. Im ersten Ausschnitt („Tschüss“) wird die museumsspezifische Aktivität (das Betrachten und die exponatbasierte Konstruktion von Wissen) suspendiert und in eine alternative, spielerische Nutzung der Exponate überführt. Im zweiten Ausschnitt („Führung“) inszeniert Aaron eine spielerische Travestie einer museumsspezifischen Aktivität, einer „Führung“.

\section{V9759, „Tschüss“}

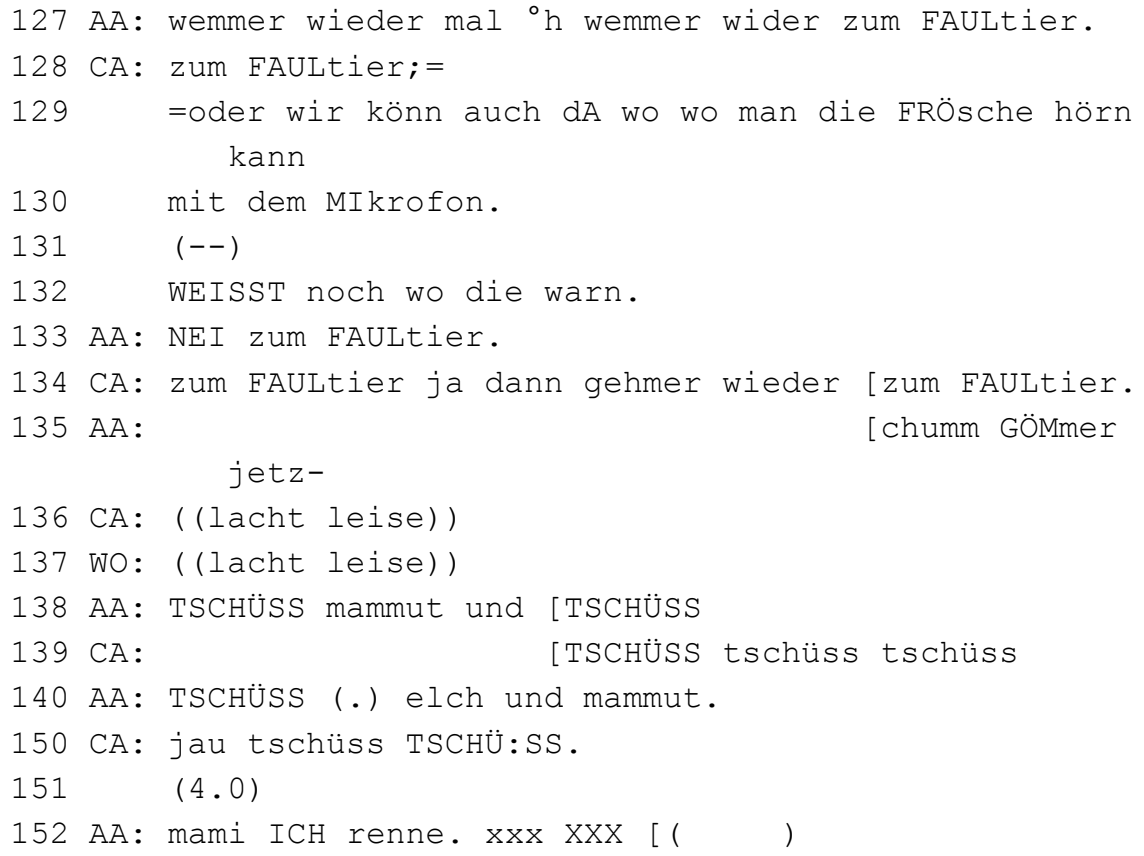




\section{CA: [nein_nein_NEIN, \\ 154 du wEIsst doch im muSEum (.) $\downarrow$ LÄUFT man. \\ $155 \uparrow \mathrm{DA} \downarrow$ RENNT man nicht.}

Aaron beendet hier die Beschäftigung mit dem Mammut, indem er vorschlägt, wieder zum Faultier zu gehen (Z. 127). Dieser Vorschlag widerspricht der in 5.2 beschriebenen charakteristischen Organisation der Bewegungen im Museum als Folge von Betrachtungen. Denn Aarons Vorschlag führt nicht zu einem nächsten Exponat, sondern zurück zu dem Exponat, das die beiden Besucher gerade vor ,Elch' und Mammut betrachtetet haben. Seine Mutter wiederholt etwas ungläubig „Zum FAULtier;“ (Z. 128) und schlägt, mit auffällig schnellem Anschluss, Alternativen vor. Aaron beharrt weiter auf dem Faultier und Caroline akzeptiert den Vorschlag ihres Sohnes mit einem Anflug komischer Resignation in der Stimme..$^{30}$ Der Junge setzt sich nun in Bewegung, und die beiden Erwachsenen - Caroline und der filmende Forscher (WO) - lachen sich einander an, womit sie sich gegenseitig das Unerwartete und damit Komische in Aarons Verhalten signalisieren.

Direkt im Anschluss (und möglicherweise ausgelöst durch das Lachen der Erwachsenen, die ja sein Handeln zu etwas Komischem gemacht haben) wechselt Aaron in eine spielerische Interaktionsmodalität. Er behandelt Mammut und Riesenhirsch (,Elch') nicht mehr, wie noch unmittelbar zuvor, als Zeichenkörper, aus deren Betrachtung Wissen über zoologische Sachverhalte erarbeitet werden kann. Vielmehr spricht er sie als Interaktionspartner an, die verabschiedet werden müssen, wenn man sich von ihnen entfernt. Zur Verabschiedung verwendet er das vertraute Tschüss (Z. 138) und gebraucht die Wörter Elch und Mammut nun nicht mehr als zoologische Kategorien, sondern als Eigennamen: „TSCHÜSS (.) elch und mammut.“ (Z. 138). Seine Mutter macht das Spiel mit (vgl. das ,alberne“ ,jau“ in Z. 150) und verabschiedet sich ebenfalls laut rufend.

In den restlichen Zeilen des Transkripts kann man verfolgen, wie die Mutter die Spielsequenz beendet und ihren Sohn wieder zum ,Ernst ${ }^{\star}$ des Museumsbesuchs zurückführt. Als nämlich Aaron einen weiteren spielerischen Akt vorschlägt - die subversive Nutzung des Ausstellungsraums zum Rennen (Z. 152), erinnert ihn die Mutter mit Nachdruck (und beinahe singend) an die geltenden Bewegungsregeln im Museum: „nein_nein_NEIN, du wEIsst doch im muSEum (.) $\downarrow$ LÄUFT man. $\uparrow$ DA $\downarrow$ RENNT man nicht.“ (Z. 153-155; s.o. 5.2).

Das zweite angekündigte Beispiel zeigt einen Fall, in dem ernste und spielerische Nutzung der Ausstellung sich überlagern. Der Ausschnitt „Führung“ stammt

30 Wobei mir in „wieder“ (Z. 134) eine Anspielung auf die Unangemessenheit der vorgeschlagenen Bewegungsrichtung - zurück, und nicht weiter - zu stecken scheint. 
aus einer früheren Phase des gleichen Besuchs. Dem Ausschnitt geht eine kurze Aushandlungssequenz voraus, in der sich Aaron und Caroline darauf geeinigt haben, als nächstes zum Mammutexponat zu gehen. (Das Mammut ist durch den Bogen hinten links im Bild zu erreichen.)
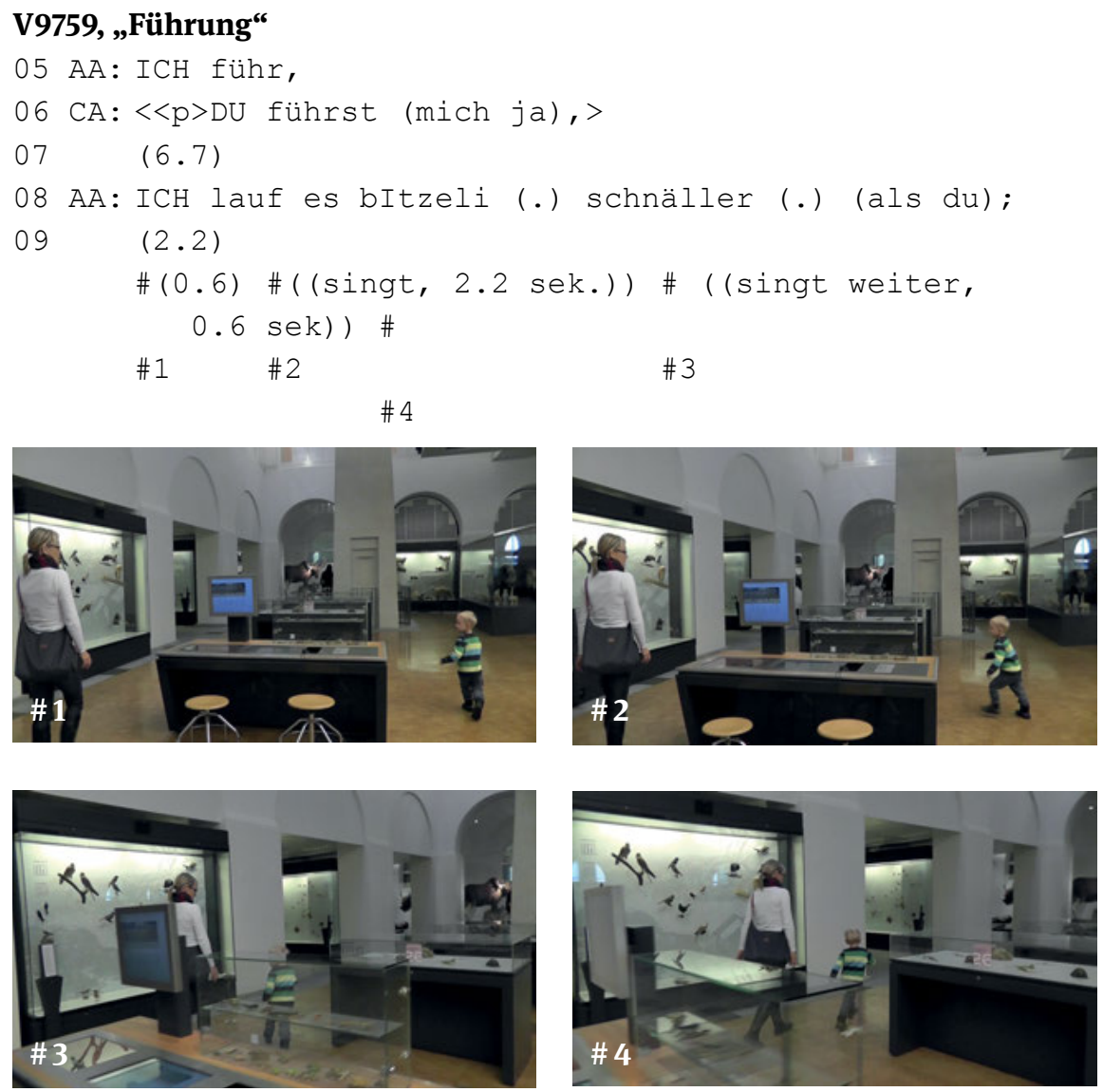

Hier vermischen sich eine museumstypische Aktivität und eine Aktivität des Spiels. Aaron schlägt seiner Mutter vor, eine museumstypische Aktivität auszuführen: Er will seine Mutter führen (,ICH führ,“, Z. 5) und seine Mutter akzeptiert diesen Vorschlag (, «<p>DU führst (mich ja),>“, Z. 6). Nun führt Aaron diese Aktivität tatsächlich aus, indem er dafür sorgt, dass er während des gesamten Gangs zum Mammut vor der Mutter bleibt.

- $\quad$ Er dreht seinen Kopf so, dass er seine Mutter im Blick behält (\#1);

- Sobald erkennbar wird, dass Caroline ihn überholen wird, beschleunigt er seinen Schritt deutlich (\#2), bis hin zu einem kleinen Sprungschritt; 
- und in dem Moment, als er sich vor seine Mutter einordnet, überprüft er ihre relative Position (\#3).

Mit seiner Beschleunigung des Schritts aber beginnt er, albern vor sich hin zu singen. Das Vorausgehen ist nun nicht mehr nur Teil des für den Museumsbesuch funktionalen Führens, sondern auch Teil des Spiels, schneller zu sein als die Mutter. Sobald er sich vor seiner Mutter eingeordnet hat, beginnt er, sein Gehen zu übertreiben, indem er rhythmisch auf den Boden stampft, wobei er seine angewinkelten Arme im Rhythmus des Gehens hin- und herschwingt. Aaron gestaltet sein Gehen also auf eine Weise, dass es gleichzeitig als Führung und als Spiel zu erkennen ist: Es ist eine Travestie der musealen Aktivität des Führens. Aarons Spiel findet nicht unabhängig vom Ausstellungsraum statt. Es gewinnt vielmehr seinen ,Witz' dadurch, dass es im Ausstellungsraum stattfindet (und nicht in den Räumen eines Kindergartens oder auf einem Spielplatz). Nur im Museumsraum kann Aarons Aktivität als Führen wahrgenommen werden und nur dort wird sein rhythmisches, von Stampfen und Gesang begleitetes Gehen zu etwas Subversivem, die Erwartungen Brechendem.

Die Analysen dieses Abschnitts zeigen, dass das Spielen nicht den gleichen Stellenwert für die Beschreibung der sozialen Praxis des gemeinsamen Museumsbesuchs hat wie das Betrachten der Exponate und die raum- und objektbasierte Herstellung von Wissen. Und dennoch sind in meinem Material Phasen spielerischer Interaktion sowie das Signalisieren von Spaß und positiven Emotionen immer wieder anzutreffen, und das gerade auch dort, wo sich die Interaktionspartner gegenseitig signalisieren, dass sie sich neues (Fach-)Wissen erarbeiten. Es lohnt sich m.E. deshalb, in einer späteren Untersuchung diesem Aspekt detaillierter $\mathrm{zu}$ betrachten.

\subsubsection{Fazit: der museale Handlungsraum}

In diesem Analyseabschnitt habe ich rekonstruiert, welche Aktivitäten die soziale Praxis des gemeinsamen Museumsbesuchs ausmachen und habe untersucht, wie die Besucher einen charakteristischen Handlungsraum hervorbringen, der ihnen die räumlichen Voraussetzungen für die Durchführung dieser Aktivitäten liefert. Im Vordergrund stand die Untersuchung der raum- und objektbasierten Herstellung von Wissen zum ausgestellten Thema - im Großteil der von mir aufgenommenen Museumsbesuche ist das die dominante gemeinsame Aktivität der Besucher.

Zunächst bin ich der Frage nachgegangen, wie sich die Konstruktion von Wissen in der Interaktion zeigt. Hier kamen einerseits Formen der expliziten The- 
matisierung von Wissen und Nichtwissen in den Blick, andererseits die Nutzung von Kategoriensystemen der ausgestellten Wissenschaftsdisziplin. Die Relevanz von Wissen wurde zudem deutlich an dem besonderen Anspruch an die Korrektheit, Präzision und argumentativen Stützung des Geäußerten - allesamt Merkmale, die in der Fachsprachen- und -kommunikationsforschung als Merkmal fachsprachlicher, speziell wissenschaftssprachlicher Kommunikation beschrieben worden sind. Schließlich habe ich nachgewiesen, dass und wie die Darstellung eines Wissensgefälles und seine Einebnung als ,Motoren“ der Gesprächsdynamik für die spezifische sequenzielle Struktur der Interaktion vor der Vitrine verantwortlich gemacht werden können.

Ausgehend von diesen Beobachtungen habe ich dafür argumentiert, die Interaktion im Museum als einen speziellen Fall von Wissenskommunikation in den Blick zu nehmen. Wissen habe ich dabei nicht als individualpsychologische Größe, sondern als kommunikativen Gegenstand untersucht: als ein von den Interaktionsbeteiligten gemeinsam in der Interaktion etablierter Status von Sachverhalten, die von den Interaktionspartnern gemeinsam als gewiss und zutreffend (häufig auch: als allgemein bekannt und als allgemein gültig) behandelt werden. Wissen heißt aus dieser Perspektive: sich in der Interaktion einen ,common ground‘ von geteilten Überzeugungen - hier: zu Sachverhalten aus der Zoologie - zu erarbeiten und diesen Status zu signalisieren.

Was aber ist nun das museumsspezifische an der interaktiven Konstruktion von Wissen, die wir in meinem Korpus beobachten konnten? Es ist die Raum- und Objektbasiertheit, die die Wissenskonstruktion in der Ausstellung auszeichnet.

Kristallisationspunkte der Wissenskonstruktion beim gemeinsamen Museumsbesuch sind die Exponate, die von den Besuchern als Zeichen aufgefasst und genutzt werden. Hörbar wird das beispielsweise, wenn Besucher, die ein einzelnes Exponat betrachten, über eine Klasse von Tieren sprechen, wenn Besucher, die vor einem unbelebten Exponat stehen, über dessen Lebensweise sprechen, als hätten sie ein lebendes Tier vor sich, oder wenn sie aktuell Merkmale an einem Exponat betrachten, aber über diese Merkmale in einer Vergangenheitsform sprechen usw.

Diese Exponate nutzen sie für ihre interaktive Generierung von fachlichem Wissen, indem sie ausgehend von individuellen Merkmalen, die sie am Exponat beobachten, allgemeine Merkmale einer vom Exponat repräsentierten Klasse ableiten. Dieser Schluss von dem, was an den Exponaten gesehen wird, zu dem, was die Exponate repräsentieren ist keinesfalls trivial. Die Verbindung zwischen dem Exponat als Zeichenkörper und dem, wofür das Exponat ,steht', ist nicht einfach in das Exponat eingeschrieben. Sie muss von den Besuchern hergestellt werden - geleitet von den Signalisierungen im Ausstellungsraum. 
Dass für die Herstellung einer Verbindung zwischen Exponat und dem ,gemeinten' Sachverhalten einiges an Expertise erforderlich ist, zeigt sich mit besonderer Deutlichkeit in Fällen, in denen einer der Besucher nicht über die dafür nötige Kompetenz verfügt. Einen solchen Fall habe ich ausführlich untersucht. Ich habe analysiert, wie die Interaktionspartner die Frage $\mathrm{zu}$ beantworten suchen, welche der wahrgenommenen Merkmale als individuelle Merkmale des ausgestellten Exponats und welche als allgemeine Merkmale der repräsentierten Klasse aufgefasst werden sollen; und ich habe rekonstruiert, wie die Besucher sich eine geteilte Überzeugung erarbeiten, welches die Klasse ist, die das Exponat konkret darstellen soll. Diese Fallanalyse hat zeigen können, wie komplex das semiotische Verhältnis zwischen dem, was in der Ausstellung wahrgenommen werden kann, und dem ,Gemeinten' sein kann: So nutzen die Besucher bisweilen mehrere Exponate als unterschiedliche, sich ergänzende Zugänge zu einer auf mehrere Zeichenkörper ,verteilten‘ Bedeutung.

Ein Teil der hier beobachteten semiotischen Komplexität liegt darin begründet, dass die Besucher die Exponate (bald alternativ, bald gleichzeitig) als Zeichen behandeln, die auf Abwesendes verweisen, und als materielle Objekte, die eine Vielzahl von sinnlichen Erfahrungen ermöglichen (von denen nur einige wenige für den Schluss auf das Bezeichnete notwendig sind). Der Doppelcharakter der Exponate zeigt sich an der spezifischen Art, mit der sie in der Interaktion behandelt werden. Die Besucher sprechen über die repräsentierten allgemeinen Sachverhalte (z.B. eine bestimmte Verhaltensweise der durch das Exponat vertretenen Gattung) als in der Wahrnehmungssituation gegenwärtig und verknüpfen mit Blicken oder deiktischen Ausdrücken die repräsentierten Sachverhalte mit dem Exponat im gemeinsamen Betrachtungsraum. Dabei können die Besucher, wie ich gezeigt habe, zwischen einer Nutzung des Exponats als Zeichen und seiner Nutzung als sinnlich wahrnehmbares, individuelles Objekt hin und herpendeln. Beide Perspektiven auf das Exponat können sich sogar überlagern, sodass das Exponat gleichzeitig als Individuum und als Stellvertreter einer Klasse von Tieren angesprochen wird.

Die Wissenskonstruktion in der Ausstellung nimmt also charakteristischerweise ihren Anfang im genauen Betrachten der Exponate, wie ich es in 5.3 beschrieben habe - aber nicht nur im Betrachten der Exponate, sondern allgemeiner: im konzentrierten Wahrnehmen des multimodalen dauerkommunikativen Angebots im Ausstellungsraum, also auch im Lesen der Texte im Ausstellungsraum, im Nutzen der Audiostationen, Manipulieren der Mikroskope, Wahrnehmen der globalen, taxonomieorientierten Anordnung der Vitrinen im Ausstellungsraum usw. Kurz: die Wissenskonstruktion im Ausstellungsraum ist objektbasiert und, per extensionem, raumbasiert; sie setzt, um mit Goodwin zu sprechen, auf der „material structure in the surround“ auf (s.o. Kapitel 3). 
Wie schon im Fall des Bewegungsraums (5.2) und des Betrachtungsraums (5.3) haben wir in der Analyse des musealen Handlungsraums beobachten können, dass und wie Erwachsene den sie begleitenden Kindern den angemessenen Umgang mit dem Ausstellungsraum vermitteln, hier: die Nutzung des Ausstellungsraums für die Konstruktion von Wissen. Dabei ging es ebenso um die Vermittlung zoologischen Faktenwissens als auch um eine grundlegende Kompetenz im Umgang mit dem kommunikativen Angebot im Museumsraum, um die Art und Weise etwa, wie man sich durch die Betrachtung der Exponate Wissen erschließen kann (s.o. „Bartgeier“) oder um die Klärung der Frage, wie das Repräsentierte und die Exponate zusammenhängen können („Knochen“).

Schließlich habe ich untersucht, ob angesichts der großen Bedeutung des Betrachtens für die Praxis des gemeinsamen Museumsbesuchs der Handlungsraum im Museum nicht einfach mit dem Betrachtungsraum zusammenfällt. Anhand zweier Fallanalysen habe ich gezeigt, dass der museale Handlungsraum als eigenständiger Raum verstanden werden muss: Besucher können nämlich den Handlungsraum aus der Vitrine ,herausziehen“ und ihn in einen „o-space“ zwischen ihren Körpern verlagern. Etwa dann, wenn sie ihren Körper als Demonstrationsobjekt nutzen, um einen in der Vitrine sichtbaren Sachverhalt am eigenen Körper zu verdeutlichen. Oder wenn sie den Blick auf die Vitrinenobjekte strategisch unterbinden, um die Gültigkeit der eigenen Vorerfahrung gegen die unmittelbare visuelle Evidenz der Exponate durchsetzen zu können. Die große Bedeutung, die beim Museumsbesuch dem Betrachten zukommt, führt dazu, dass der Handlungsraum vor der Vitrine leicht übersehen werden kann. Doch ereignen sich dort ebenfalls besuchsrelevante Aktivitäten - nur dass diese in der Regel auf den Videos weniger auffällig sind, weil sie weniger sichtbar als vielmehr hörbar sind: Voraussetzung für die museale Wissenskonstruktion ist nicht nur, dass die Besucher die Exponate betrachten können, sondern eben auch, dass sie sich bei der sprachlichen Erarbeitung von deren Bedeutung gegenseitig hören können, denn nur so können sich die Besucher interaktiv einen ,common ground' der geteilten Überzeugungen über die ausgestellten Themen erarbeiten. Der charakteristische Handlungsraum für die soziale Praxis des gemeinsamen Museumsbesuchs besitzt daher zwei ,Ausbuchtungen', die eine, vor allem visuelle, die auf die Exponate fokussiert ist, und eine zweite, vorrangig auditive, die den verbalen Austausch unter den Besuchern herstellt (und die sicherlich für die in 5.2 beobachtete Tatsache mitverantwortlich ist, dass die Besucher sich in aller Regel nicht weit voneinander entfernen).

Damit habe ich im Abschnitt 5.4 die zwei Grundpfeiler der sozialen Praxis des gemeinsamen Museumsbesuchs rekonstruiert: das gemeinsame, detailorientierte Betrachten der Exponate (und insgesamt: des multimodalen Kommunikationsangebots im Ausstellungsraum) einerseits und die raum- und objektbasierte 
Wissenskonstruktion andererseits. Diese beiden Aktivitäten sind eng aufeinander bezogen, aber dennoch prinzipiell unabhängig voneinander. So ist in meinem Korpus - das ist sicherlich spezifisch für den untersuchten Museumtyp - das Betrachten meist funktional für die Konstruktion von Wissen. Und dennoch: Das Betrachten erschöpft sich nicht in seiner Nützlichkeit für die Wissenskonstruktion: Die Exponate sind eben mehr als nur Zeichen, sie sind materielle Objekte, die einen Überschuss an Wahrnehmungen anbieten, von denen nur die wenigsten semiotisch genutzt werden - hierin scheint mir das zu liegen, was oft als die spezifische ,Museumserfahrung` am ,authentischen Objekt‘ (Korff) bezeichnet worden ist. Die Wissenskonstruktion ihrerseits fußt zwar, wie ich gezeigt habe, charakteristischerweise auf dem, was im Ausstellungsraum wahrgenommen und gelesen werden kann, aber gleichzeitig können die Besucher (Vor-)Wissen aus anderen Quellen in ihre Interaktion im Ausstellungsraum ,einspeisen“.

\subsection{Fazit: die Kommunikation in der Ausstellung}

In diesem Kapitel habe ich ungeführte, authentische Museumsbesuche analysiert, die ich per Video dokumentiert habe, und so mit den Methoden der Konversationsanalyse die Elemente rekonstruiert, die die soziale Praxis des gemeinsamen Museumsbesuchs ausmachen.

Anstatt das Interaktionsgeschehen vor der Folie der semiotisch-textlinguistischen Analysen des vorausgegangenen Kapitels zu betrachten und die Analyse auf die Frage zu beschränken, ob die Besucher die dort identifizierten Bedeutungspotenziale nutzen oder nicht, hat dieses Kapitel ganz neu angesetzt und die grundlegende Frage gestellt, wie die Besucher ihre Interaktion als gemeinsamen Museumsbesuch hervorbringen. Diese Entscheidung hatte zwei Konsequenzen: Zum einen konzentriert sich die Analyse ganz auf die Untersuchung körpergebundener Modi wie (gesprochener) Sprache, Gestik, Blick, Bewegung usw., die die Interaktionsbeteiligten ,in die Situation mitbringen'. Zum anderen ist die Untersuchung nicht den in Kapitel 3 identifizierten kommunikativen Aufgaben des Ausstellungsraums gefolgt, sondern hat sich an den interaktiven Aufgaben orientiert, die sich die Besucher selbst signalisieren, wenn sie ihre Interaktion in ihrer räumlichen Umwelt verankern (,situieren“) und damit diese räumliche Umwelt zugleich als Ausstellungsraum konstruieren.

Die Frage, wie die Besucher den Ausstellungsraum für ihren gemeinsamen Museumsbesuch nutzen, habe ich auf drei Teilfragen heruntergebrochen, die sich an den von Hausendorf (2010) identifizierten Teilaufgaben der „Situierung“ orientieren: der interaktiven Herstellung des „Bewegungs-“, „Wahrnehmungs-“ und „Handlungsraums“. Indem die Interaktionsbeteiligten diese Aufgaben bearbei- 
ten, bringen sie die räumlichen Voraussetzungen für ihre Interaktion hervor (den „Interaktionsraum“) und verankern sie in ihrer räumlichen Umwelt. Wie dieser Interaktionsraum beim Museumsbesuch hergestellt wird, habe ich mit konversationsanalytischen Methoden untersucht. Dabei bin ich von der feingliedrigen Analyse einzelner Fälle ausgegangen und habe die Musterhaftigkeit der jeweils interessierenden Phänomene über den Vergleich zu ähnlich gelagerten Fällen und die Kontrastierung zu „deviant cases“ herausgearbeitet.

Die Analysen zu jeder Teilaufgabe der Situierung erfolgte in zwei Schritten. Nachdem zunächst jeweils untersucht wurde, wie die Besucher ihre Bewegungen, Blicke und Handlungen im Ausstellungsraum als gemeinsame hergestellt haben, konzentrierte sich der zweite Schritt auf die Rekonstruktion der Musterhaftigkeit der Raumherstellung für den gemeinsamen Museumsbesuch. Wenn im ersten Schritt gefragt wurde „Wie organisieren die Besucher ihre Bewegungen, dass ihre Anwesenheit im Museum als eine gemeinsame zu erkennen ist?“, lautete die Frage im zweiten Schritt: „Wie gestalten die Besucher diese gemeinsamen Bewegungen so, dass sie als Bewegungen im Rahmen eines Museumsbesuchs erkennbar sind?“ usw. Diesem Vorgehen liegt die Idee zugrunde, dass eine musterhafte Realisierung der drei Teilaufgaben der Situierung dafür verantwortlich ist, dass ein Raum als ein bestimmter Raum hergestellt und (wieder)erkannt wird. Anders gesagt: indem die Besucher einen Interaktionsraum hervorbringen, der für den Museumsbesuch angemessen und funktional ist, machen sie ihre räumliche Umwelt als Ausstellungsraum sichtbar.

Die Rekonstruktion der interaktiven Prozesse der Raumkonstitution im Ausstellungsraum erbrachte folgende Ergebnisse.

Für die museumsspezifische Herstellung des Bewegungsraums spielen die Exponate eine zentrale Rolle. Exponate bilden legitime Ziele für die Bewegungen durch den Ausstellungsraum, die Identifikation von Exponaten kann dazu genutzt werden, die Bewegungen von anderen zu beeinflussen (in dem Sinn, dass sie sich dem identifizierten Exponat nähern) oder eigene Bewegungen zu motivieren. Die Untersuchung hat gezeigt, dass streng genommen nicht die Exponate den Endpunkt der Bewegungen durch den Raum darstellen, sondern eine Zone vor den Exponaten oder in der Nähe der Exponate, die ich „Verweilzone“ genannt habe, weil die Besucher in diesen Zonen lange statisch verharren. Die Lage und die Gestalt dieser Verweilzonen ergibt sich aus den Möglichkeiten der Betrachtung der Exponate, die von der Verweilzone aus jeweils ermöglicht werden (s.u. Wahrnehmungsraum). Ein wichtiger Aspekt der Museumsspezifik der Bewegungen durch den Ausstellungsraum besteht darin, dass die Bewegungen in stationäre Phasen (Verharren in der Verweilzone vor einem Exponat) und mobile Phasen (auf dem Weg zum nächsten Exponat) gegliedert sind, die zyklisch aufeinanderfolgen. Aus diesem Wechsel von stationären und mobilen Phasen ergibt 
sich das Bewegungsmuster des „Rundgangs“, das - wie ich gezeigt habe - gegen Abweichungen verteidigt wird und eine spezifische Deixis des Weiter und Zurück generiert. Als sekundär für die Beschreibung der Museumsspezifik der Besucherbewegungen stellte sich dagegen das gemessene Schreiten heraus, das in der Literatur oft als charakteristisch für den Museumsbesuch erwähnt wird. Die Untersuchung zweier kontrastierender Fälle zeigte: Ein langsames Gehen, das nicht an Exponaten orientiert ist, ist schwerer als Museumsbesuch verstehbar als ein schnelles Gehen, bei dem die Interaktionsbeteiligten ihre Orientierung an den Exponaten demonstrieren.

Auch bei der Herstellung des musealen Wahrnehmungsraums spielen Exponate eine wichtige Rolle (aber auch andere semiotische ,Hotspots' im Ausstellungsraum (Objektkennungen, Vitrinentexte oder die Vitrine als Gliederungseinheit). Mit diesen als Zentrum konstruieren die Interaktionspartner beim gemeinsamen Museumsbesuch ihren geteilten Wahrnehmungsraum (den ich den „musealen Betrachtungsraum“ genannt habe). Diese Konzentration auf den musealen Betrachtungsraum heißt aber auch, dass sich die Interaktionspartner überwiegend nur über ihre periphere Sicht wahrnehmen. Dabei ist auch das ,Timing‘ ihrer Blicke und die geringe Häufigkeit des Blickkontakts eine auffällige Besonderheit der Präsenz im musealen Wahrnehmungsraum. Schließlich habe ich das genaue, am Detail orientierte Sehen als Besonderheit des musealen Betrachtungsraums herausgearbeitet. Die Besucher geben sich nicht mit einer einfachen Sichtbarkeit der Exponate zufrieden. Zum ,Museumsblick' gehört, dass er auf das genaue visuelle Erfassen der Exponate abzielt. In einer Reihe von Analysen habe ich gezeigt, mit welchem Aufwand die Besucher diesen spezifischen Blick auf die Exponate herstellen, gerade auch wenn Erwachsene Kinder mit der Nutzung des Ausstellungsraums vertraut machen. Damit sind die spezifischen Elemente des musealen Betrachtungsraums benannt: Es handelt sich beim musealen Betrachtungsraum um die spezifische Ausprägung eines interaktiv hergestellten gemeinsamen Wahrnehmungsraums, der nicht auf die gegenseitige Wahrnehmung der Interaktionspartner, sondern auf das intensive, möglichst kein Detail ,verschenkende“ Betrachten von Exponaten (und anderen Elementen des multimodalen Kommunikationsangebots der Ausstellung) ausgerichtet ist. Dieser museale Betrachtungsraum ist derart musterhaft, dass wir in den Personen, die auf den Standbildern abgebildet sind, sofort und unzweifelhaft Museumsbesucher bei der Betrachtung von Exponaten erkennen.

Bei meiner Analyse des musealen Handlungsraums schließlich stand der Aspekt der Wissenskonstruktion im Vordergrund. Die interaktive Konstruktion von Wissen zum ausgestellten Fachgebiet und seinen Themen durchzieht die Besucherinteraktion im Museum wie ein roter Faden, weshalb ich den gemeinsamen Museumsbesuch auch als Fall von „Wissenskommunikation“ untersucht 
habe. Dabei bin ich von einem Wissenskonzept ausgegangen, dass Wissen nicht individualpsychologisch versteht, sondern als eine geteilte Überzeugung vom Zutreffen bestimmter Aussagen im Gespräch. Diese Sicht von Wissen hat den Blick auf die interaktiven Verfahren gelenkt, mit denen die Museumsbesucher im Ausstellungsraum Wissen erarbeiten. Es stellte sich heraus, dass die lokale Generierung von Wissen in der Ausstellung charakteristischerweise vom Sehen zum Wissen verläuft. Mit anderen Worten: die Interaktionsteilnehmer nehmen individuelle Merkmale an Exponaten wahr und nutzen diese Wahrnehmungen, um auf auf allgemeine Merkmale von durch die Exponate repräsentierten Klassen zu schließen. Dieser Umgang mit den Exponaten, der sie als Zeichen nutzt, die für etwas anderes, Abwesendes, stehen, ist für das Funktionieren der Kommunikation in der Ausstellung grundlegend. Doch der Übergang vom Sehen zum Wissen ist, wie ich gezeigt habe, keineswegs trivial. Denn der Zusammenhang zwischen dem Exponat als Signifiant und seinem Signifié ist nicht in das Exponat eingeschrieben, sondern muss von den Besuchern aktiv konstruiert werden. Ich habe anhand von einigen Ausschnitten aus meinem Korpus dokumentiert, wie diese Konstruktion vor sich gehen kann und welche komplexen semiotischen Verhältnisse sich unter anderem daraus ergeben, dass die Exponate mehr sind als nur Zeichen, sondern gleichzeitig auch Objekte mit einer eigenen Materialität, die einen Überschuss an Wahrnehmungs- und Erfahrungsmöglichkeiten eröffnen (man denke an Bühlers ,apperzeptive Ergänzung“ oder Assmanns „wilde Semiose“). Abschließend habe ich zu bestimmen versucht, welche Aktivitäten für den gemeinsamen Museumsbesuch als konstitutiv zu gelten haben. In diesem, dem Handlungsraum gewidmeten Teilkapitel hat sich gezeigt, dass die Konstruktion von Wissen beim gemeinsamen Museumsbesuch auf dem in vorausgegangenen Teilkapitel rekonstruierten musealen Sehen aufbaut. Die genauere Bestimmung des semiotischen Verhältnisses von Exponaten und den Sachverhalten, die die Besucher von der Betrachtung der Exponate ableiten, hat indes gezeigt, dass sich die Relevanz des Betrachtens nicht in seiner dienenden Funktion für das Konstruieren von Wissen erschöpft: Wie in der Museumsliteratur immer wieder beschrieben, kommt dem Betrachten der Exponate ein ganz eigener Wert zu, der sich aus den Wahrnehmungs- und Erfahrungsmöglichkeiten speist, die das Exponat aufgrund seiner Materialität eröffnet.

Indem ich rekonstruiert habe, wie die Besucher ihren Interaktionsraum (über seine drei Teilräume Bewegungs-, Wahrnehmungs- und Handlungsraum) für den gemeinsamen Museumsbesuch herstellen, habe ich gleichzeitig die wesentlichen Züge davon beschrieben, was die soziale Praxis des ,gemeinsamen Museumsbesuchs“ ausmacht. Dass ist nur möglich, weil der Umgang mit der räumlichen Umwelt für die Beschreibung der sozialen Praxis des gemeinsamen Museumsbesuch zentral ist. Das ist offensichtlich, was mit der Raum- 
gebundenheit von Kommunikation, hier konkret: der Raumgebundenheit der Interaktion im Ausstellungsraum, gemeint ist.

Damit sind wir bereits bei den Einsichten in allgemeinere Zusammenhänge, die die Analysen dieses Kapitels ermöglichen, und die über den Fall Museumsbesuch hinausgehen.

Die Analysen ermöglichen nicht nur ein Verständnis des Begriffs Raumgebundenheit, sondern auch einen Einblick in den Zusammenhang von Interaktionsräumen und der räumlichen Umwelt. Die Analysen dieses Kapitels scheinen mir zu belegen, dass die Herstellung der räumlichen Umwelt als Ausstellungsraum sich aus der spezifischen, auf den Museumsbesuch abgestimmten Gestaltung des Bewegungs-, Wahrnehmungs- und Handlungsraums ergibt; in dem Sinne nämlich, dass der musterhaft geformte Interaktionsraum den Ausstellungsraum ,indiziert" (im Sinne der Kontextualisierungsidee). Dieser Zusammenhang von Interaktionsraum und räumlicher Umwelt kann meines Erachtens die Grundlage von Analysen werden, die genauer zu bestimmen versuchen, was mit der oft schwammigen Rede von der „Herstellung von Raum“ in der Interaktion gemeint ist (wenn damit nicht einfach der Interaktionsraum gemeint ist).

Darüber hinaus haben die Analysen die analytische Fruchtbarkeit des interaktiven Wissenskonzepts belegt. Zwar kann man mit dieser Sicht des Gegenstands Wissen keine Aussagen über die mentalen Vorgänge des Lernens im Museum treffen. Doch kann man etwas über die Verfahren erfahren, die Menschen nutzen, wenn sie sich gemeinsam Wissen erarbeiten (hier: Wissen, dass sie in den wissenschaftlichen Kontext der Zoologie verorten). Und wir können untersuchen, wie genau sie in diesem Prozess Elemente ihrer räumlichen Umwelt als Zeichen nutzen, von denen sie allgemeine Aussagen ableiten.

Auch im Hinblick auf den Aspekt der Multimodalität lassen sich aus den Analysen dieses Kapitels Einsichten in allgemeinere Aspekte von Kommunikation gewinnen. So konnten wir sehen, wie die Interaktionsbeteiligten zur Herstellung jedes einzelnen der drei Teilräume des musealen Interaktionsraums mehrere körpergebundene Modi zugleich einsetzen: Bei der Herstellung des musealen Bewegungs- und Betrachtungsraums spielten eben nicht nur Bewegungen und Blicke eine Rolle, sondern beispielsweise auch Sprache, etwa bei der Definition von museumsspezifischen Bewegungs- und Betrachtungszielen. Schließlich konnten wir beobachten, dass die multimodalen Ressourcen, die die Museumsbesucher in ihrer Interaktion einsetzen, auf eine räumliche Umwelt treffen, die ihrerseits voll ist von multimodalen Zeichen.

Das folgende Analysekapitel (Kapitel 6) ist genau dieser „doppelten Multimodalität“ der Ausstellungskommunikation gewidmet, bei der eine Vielzahl körpergebundener Ausdrucksressourcen auf eine mindestens ebenso große Zahl unterschiedlicher raum- und objektbasierter Zeichen trifft. Die Analysen dieses 
dritten und letzten Analysekapitels sollen die Frage beantworten, wie die Multimodalität des kommunikativen Angebots im Ausstellungsraum von den Besuchern eingebettet wird in ihre Interaktion im Ausstellungsraum, und damit einen Weg aufzeigen, wie die semiotisch-textlinguistische Raumanalyse und eine konversationsanalytische Untersuchung der Nutzung des Raums in der Interaktion integriert werden können. 